\title{
Developmental patterning function of GNOM ARF-GEF mediated from the plasma membrane
}

Maciek Adamowski, Ivana Matijević, and Jiří Friml*

Institute of Science and Technology Austria, Am Campus 1, 3400 Klosterneuburg, Austria

*jiri.friml@ist.ac.at

\begin{abstract}
The GNOM (GN) Guanine nucleotide Exchange Factor for ARF small GTPases (ARF-GEF) is among the best studied trafficking regulators in plants, playing crucial and unique developmental roles in patterning and polarity. The current models place GN at the Golgi apparatus (GA), where it mediates secretion/recycling, and at the plasma membrane (PM) presumably contributing to clathrin-mediated endocytosis (CME). The mechanistic basis of the developmental function of GN, distinct from the other ARF-GEFs including its homologue GNOM-LIKE1 (GNL1), remains elusive. Insights from this study redefine the current notions of GN function. We show that GN, but not GNL1, localizes to the PM at long-lived structures distinct from clathrin-coated pits, while CME and secretion proceed normally in gn knockouts. The functional GN mutant variant GNfewerroots, absent from the GA, suggests that $\mathrm{PM}$ is the major place of $\mathrm{GN}$ action responsible for its developmental function. Following inhibition by Brefeldin A, GN, but not GNL1, relocates to the PM likely on exocytic vesicles, suggesting selective molecular associations. A study of GN-GNL1 chimeric ARF-GEFs indicate that all GN domains contribute to the specific GN function in a partially redundant manner. Together, this study offers significant steps towards the elucidation of the mechanism underlying unique cellular and development functions of GN.
\end{abstract}

\section{Introduction}

ARF small GTPases function in eukaryotic endomembrane systems by recruiting to intracellular membranes various effectors necessary for trafficking processes, especially for vesicle formation (reviewed in Donaldson and Jackson, 2011; Jackson and Bouvet, 2014; Singh and Jürgens, 2017; Yorimitsu et al., 2014). ARFs act as GTP hydrolysis-dependent molecular switches, and their cycles of activation and deactivation are controlled by ARF regulators, GEFs (Guanine nucleotide Exchange Factors) and GAPs (GTPase Activating Proteins), respectively. In Arabidopsis thaliana, the ARF small GTPase machinery functions in fundamental secretory activity at the level of Golgi apparatus (GA) and the trans-Golgi Network (TGN). The regulation of GA function requires the activity of the ARF-GEF GNOM- 
LIKE1 (GNL1) assisted by EMB30/GNOM (GN) (Richter et al., 2007; Teh and Moore, 2007), and of the ARF-GAPs AGD6-10 (ARF-GAP DOMAIN6-10) (Min et al., 2007, 2013). The exocytic and vacuolar traffic originated from the TGN employs BIG class ARF-GEFs (Tanaka et al., 2009; Richter et al., 2014; Xue et al., 2019) and the ARF-GAP AGD5 (Stefano et al., 2010; Sauer et al., 2013). Notably, besides acting in these basic trafficking pathways, the ARF machinery has been ascribed a prominent function in patterned development controlled by the plant hormone auxin (Weijers et al., 2018). This function of the ARF machinery is, to some degree, genetically separable from the basic secretory function, and is mostly associated with the activity of the ARF-GEF GN (reviewed in Richter et al., 2010) and the ARF-GAP VAN3 (Koizumi et al., 2005, 2000; Deyholos et al., 2000; Sieburth et al., 2006; Steynen and Schultz, 2003; Naramoto et al., 2009, 2010, 2018; Adamowski and Friml, 2021).

The gn/emb30 mutants were isolated in forward genetic screens aimed at the identification of early pattern formation mutants in $A$. thaliana (Meinke, 1985; Mayer et al., 1991, 1993; Shevell et al., 1994). Further studies with the use of moderate and weak gn alleles revealed that GN acts also at later stages of plant development (Geldner et al., 2004; Okumura et al., 2013). gn mutant phenotypes include the development of fused cotyledons during embryogenesis, failure of root development at the basal embryonic pole, disorganized vascular tissue formation, defects in organization of lateral root primordia, and deficiencies in tropisms (Mayer et al., 1991, 1993; Shevell et al., 1994; Geldner et al., 2003, 2004; Okumura et al., 2013; Koizumi et al., 2000; Miyazawa et al., 2009; Moriwaki et al., 2013; Verna et al., 2019). Polarities of PIN1 auxin efflux carriers (reviewed in Adamowski and Friml, 2015) are not coordinated between cells in gn embryos (Steinmann et al., 1999). An often-cited mechanism of GN action proposes that GN is required for the exocytic delivery of PIN1 to the polar (esp. basal) domain at the plasma membrane (PM) as part of PIN1's constitutive recycling between the PM, and an endosome (Geldner et al., 2001, 2003; Kleine-Vehn et al., 2008) and for PIN polarity switches during processes such as gravitropism (Kleine-Vehn et al., 2010; Rakusova et al., 2011). Beside PINs, the function of GN in auxin-mediated development encompasses other molecular targets, as well. The PM recruitment of D6 PROTEIN KINASE (D6PK), a protein kinase activating auxin efflux activity of PINs at the basal domain of the PM (Willige et al., 2013), is dependent on GN (Barbosa et al., 2014). In the context of vascular tissue patterning, GN has been suggested to influence nuclear auxin signalling, as indicated by phenotypic similarities between gn mutants and plants concomitantly deficient in auxin signalling and polar auxin transport (Verna et al., 2019). Finally, study of gn knockouts (Shevell et al., 2000) as well as recent forward genetic screening (Wachsman et al., 2020) identifies a function of GN in the regulation of cell wall 
composition, which may be a part of the mechanism by which GN controls developmental patterning, as well.

Altogether, studies of GN demonstrate its major function in embryonic and postembryonic plant body development. By multiple and only partially understood mechanisms, tightly linked with the control of cellular polarity, GN's activity leads to the establishment of organized polar auxin transport streams instructing developmental patterning events. This activity represents a unique modification of the conserved cellular ARF machinery acting in fundamental trafficking processes. To perform this function, GN acquired novel and so far elusive molecular features, absent in its GBF1-type ARF-GEF homologue GNL1 (Richter et al., 2007). Better understanding of the molecular and cellular bases of the unique GN function would be an important step in the unravelling of molecular mechanisms of plant developmental patterning as a whole, and would illustrate how the endomembrane system became modified in the course of evolution to provide for the requirements of a complex plant body and its adaptive life strategy.

Here, we characterize in detail the function of GN considering its molecular nature as an ARF small GTPase regulator in the cellular endomembrane system. We investigate the subcellular site of action of GN required for its role in developmental patterning, as well as GN's action in endo- and exocytic processes. We utilize direct comparisons with GNL1 to obtain precise information about the molecular function specific to GN. The internal determinants of GN function within the ARF-GEF protein itself are studied through the use of GN-GNL1 chimeric ARF-GEFs. Our findings constitute a significant step in the elucidation of the molecular mechanism underlying the unique GN function in developmental patterning.

\section{Results}

\section{GN, but not GNL1, localizes to the PM at structures of unknown nature}

Within the cell, the ARF-GEF GN has been originally placed at a recycling endosome (RE; Geldner et al., 2003), based on its co-localisation with endosomal markers and with the FM4-64 fluorescent endocytic tracer dye (Jelínková et al., 2010) at the core of the so-called "BFA body", an endomembrane compartment aggregation formed in the root cells of $A$. thaliana by chemical inhibition of ARF-GEFs with Brefeldin A (Robinson et al., 2008; Robineau et al., 2000). Further studies in chemically undisturbed conditions, with the use of high-resolution imaging techniques, indicated that GN localises to the GA together with its homologue GNL1, a site of action typical for GBF1-type ARF-GEFs in non-plant systems (Naramoto et al., 2014). In addition to the localisation to the GA, GN is variably found at the $\mathrm{PM}$, where it was reported to colocalize with the endocytic vesicle coat protein clathrin 
(Naramoto et al., 2010). Taken together, GN is described as localized to the GA and to the $\mathrm{PM}$, presumably participating in clathrin-mediated endocytosis (CME) at the latter.

We first analysed in more detail the cellular sites of action of $\mathrm{GN}$, and compared it with the localization of GNL1. For this purpose we generated new fluorescent reporter lines for GN and GNL1, expressed under GN promoter (GN pro:GN-GFP and $G N_{\text {pro: }}$ GNL1-GFP). We verified the functionality of the $\mathrm{GN}$-expressing construct by the complementation of a $\mathrm{gn}$ null mutant allele $g n^{S A L K} 103014\left(g n^{s}\right.$ in the following; Figure 1-figure supplement 1A, 1B, 1E). As a sensitive readout of GN function, we analyzed root hair positioning and identified no shift towards the apical cell side, characteristic for partial gn loss of function (Fischer et al., 2006), in the complemented mutant (Figure 1-figure supplement 1D). We also confirmed the observation that expression of additional copies of GNL1 cannot complement the gn mutant (Figure 1-figure supplement 1C; Richter et al., 2007), verifying that GNL1 does not possess a function in developmental patterning shared with $\mathrm{GN}$, an important assumption of the present work.

Having confirmed this, we compared the subcellular localizations of $G N_{\text {pro: }}: G N-G F P$ and $G N_{\text {pro: }} G N L 1-G F P$, expressed in wild type background, by confocal laser scanning microscopy (CLSM) in the epidermis of seedling root apical meristems (RAMs). In two independent transgenic lines expressing each construct, with settings aimed at the detection of weak signals, GN-GFP was observed at the PM in all seedlings analysed, while GNL1GFP was never found localizing to the PM (Figure 1A and Figure 1-figure supplement 2A). Beside these differences, a larger portion of GN was observed in the cytosol relative to punctate signals representing GA, in contrast to a strong binding of GNL1 to these organelles. In comparison with previously reported observations (Naramoto et al., 2010), our new $G N_{\text {pro: }}$ GN-GFP lines suggest that the localization of GN to the PM is a typical, rather than a rare, phenomenon.

Next, we compared the localizations of GN-GFP and GNL1-GFP using Total Internal Reflection Fluorescence (TIRF) microscopy in the early elongation zone of seedling roots (Figure 1B and 1C, Figure 1-figure supplement 2B, Video 1 and 2). This method is used for the observation of fluorescent reporters localized at the PM and in the cytosol directly underneath, at high magnification. In two independent transgenic lines expressing each construct, we observed the localization of both ARF-GEFs to the GA and often to weakly fluorescent, small and dynamic structures, probably residing in the cytosol. In turn, only GN, but not GNL1, was found at PM-localized structures characterized by a relatively bright fluorescence and often by high stability over time (Video 1, Figure 1B and 1C, Figure 1figure supplement 2B). In 100s-long time lapses, many of the structures could be seen docked in the PMs throughout the whole time course of observation, indicating that typical 
residence life times exceeded $100 \mathrm{~s}$. The structures exhibited limited lateral diffusion in plane of the PM (Figure 1C, Figure 1-figure supplement 4A). These structures represent a subcellular localization site specific for GN, but not for GNL1, and as such, are a potential site of action connected with the exclusive GN's function in developmental patterning.

The localization of GN at the PM is strongly enhanced following its inhibition by BFA

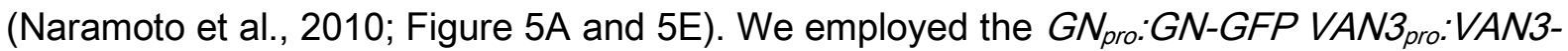
$m R F P$ double reporter line and observed an almost complete disappearance of the stable GN-positive structures following a treatment with BFA at $50 \mu \mathrm{M}$ for $1 \mathrm{~h}$. Instead, BFAinhibited GN appeared as very dense punctate signals, characterized by rapid dynamics at the PM (Figure 1D, Figure 1-figure supplement 4B and Video 3 and 4). These highly dynamic signals did not colocalize with similar, dense and dynamic PM signals of the ARFGAP VAN3 (Figure 1-figure supplement 3 and Video 5-7). In chemically undisturbed conditions, GN-positive structures also do not colocalize with the PM signals of VAN3 (Adamowski and Friml, 2021).

Taken together, our new observations of GN localization patterns, in direct comparison with its close homologue GNL1, point to the existence of structures of unknown nature present at the PM, which may be the sites of action of GN in developmental patterning.

\section{The functional GNiwr mutant variant localizes solely to the PM}

Given the notion that the PM may be the site of action relevant for the developmental patterning function of $\mathrm{GN}$, we were interested in analysing a previously described allele of GN, FEWERROOTS (GN ${ }^{\text {fwr }}$; Okumura et al., 2013). fwr is a weak gn mutant allele, carrying a single amino acid substitution in the HDS2 (HOMOLOGY DOWNSTREAM OF SEC7 2) domain. The mutant develops relatively normally, with the exception of a reduced ability to form lateral roots (LRs; Okumura et al., 2013), and a moderate phenotype in vascular network patterning (Verna et al., 2019). Thus, GNiwr may be considered a relatively wellfunctioning GN protein variant. Interestingly, it was reported that GN fwr localizes to the PM, instead of the GA, in undisturbed conditions (Okumura et al., 2013). To understand the relevance of GN's localization to the PM, we compared directly the localization patterns and functionality of GN fwr and of wild type GN.

First, we tested the functionality of GNiwr-GFP protein fusions by the complementation of $g n^{s}$ mutants with a $G N_{\text {pro: }}$ GNwr-GFP construct. Two independent $g n^{s}$ $G N_{\text {pro: }} G N^{\text {fwr_G }}$ GFP lines were phenotypically indistinguishable from control $g n^{s} G N_{\text {pro: }} G N-G F P$ lines, and from wild type plants, during seedling development and at adult stages (Figure $2 \mathrm{~A}$ and $2 \mathrm{~B}$, Figure 1-figure supplement $1 \mathrm{E}$ ), and presented normal root hair outgrowth 
positioning at basal cell sides (Figure 1-figure supplement 1D). These observations demonstrate that $\mathrm{GN}^{\mathrm{fw}}{ }^{\mathrm{i}}$ is a highly functional $\mathrm{GN}$ variant.

Next, using TIRF, we directly compared the localization patterns of GN-GFP and GNfwr-GFP, expressed in complemented mutant backgrounds, in the epidermis of etiolated hypocotyls and of the early elongation zone of roots (Figure 2C-E and Video 8). Like GN-

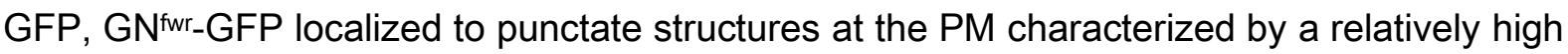
signal intensity and high stability. The density of GNiwr-GFP structures at the PM was slightly higher than those of GN-GFP, while their lateral displacement was similar to wild type GN (Figure 1-figure supplement 4A and 4C). Interestingly, in contrast with GN-GFP, GN ${ }^{\text {fwr-GFP }}$ was never found localized to the GA in neither of the organs. Considering that the observations were made in successfully complemented gn null mutants, the localization of GN'fwr-GFP to the structures at the PM, but not to the GA, constitutes strong evidence that the PM-localized structures are the site of $\mathrm{GN}$ action responsible for its function in developmental patterning.

We additionally assessed localization patterns of GN-GFP and GN fwr-GFP during root hair initiation, to ascertain the validity of our observations in a context of a known GN function carried out in the epidermis, i.e., root hair positioning (Fischer et al., 2007) and correctly mediated by the used fluorescent protein fusions (Figure 1-figure supplement 1D). We employed TIRF imaging at basal ends of young trichoblasts, and documented localization of GN-GFP and GNfwr-GFP to PM-localized structures, presumably during the process of root hair positioning (Figure 2-figure supplement $1 \mathrm{~A}$ and $1 \mathrm{~B}$ ).

The difference in phenotypes between gn knockout mutants complemented by GNfwrGFP expression, where normal phenotypes were found by all applied criteria, and the original fwr mutant (Okumura et al., 2013), might be explained by a higher level of GNiwr protein expression, or altered expression patterns, in the transgenic lines. In our view, even if, following the fwr phenotype, GNfwr is considered as an incompletely effective GN variant, unable of promoting normal LR formation or correct vascular patterning, but functional in embryonic and most of post-embryonic development, the presented argument that PM, rather than the GA, is the site of $G N$ action in developmental patterning, remains valid.

In summary, the comparison of subcellular localizations of GN and GNL1, as well as the localization pattern of the GN mutant variant $G N^{\text {fwr }}$, suggest that the developmental function of $\mathrm{GN}$ is mediated on the cellular level from structures of unknown nature localized at the PM.

GN-positive structures at the PM are distinct from clathrin-coated pits and CME functions in the absence of GN 
The localization of GN at the PM was previously associated with a proposed function of GN, and the ARF machinery as a whole, in CME (Naramoto et al., 2010). Yet, the GN-positive PM-localized structures do not resemble clathrin-coated pits (CCPs) forming at the PM in several characteristics: typically very long life times at the PM, lateral mobility not observed with CCPs, and low density. To clarify this discrepancy, we verified the co-localization patterns of GN and clathrin at the PM by capturing TIRF time lapses of the $G N_{\text {pro: }}$ GN-GFP 35S pro: CLC-mKO (CLATHRIN LIGHT CHAIN-monomeric Kusabira Orange) double marker line, which marks the CLC subunit of the clathrin coat. In TIRF time lapses, the GN-positive structures at the PM were clearly distinct from CCPs labelled by CLC-mKO (Figure 3A, 3B, Video 9-11). As a comparison, we performed TIRF co-localization of fluorescent reporters for two components of CCPs, TPLATE-GFP and AP2A1-TagRFP, where co-localization was readily detected (Figure 3-figure supplement 1; Gadeyne et al., 2014). Together, the observations are not supportive of a functional link between GN function at the PM, and CME.

Next, as a test of GN requirement in $\mathrm{CME}$, we crossed fluorescent reporter protein fusions for clathrin (CLC2 pro:CLC2-GFP) and for the TPLATE complex, a component of CCPs (LAT52 pro:TPLATE-GFP; Gadeyne et al., 2014) into $g n^{s}$ mutant background, and used TIRF microscopy to assess the activity of CME in the absence of GN function. We imaged the epidermis of the middle region of etiolated gn seedlings, corresponding to the hypocotyl, and as a control, we used etiolated hypocotyls of wild type siblings from the segregating $g n^{s}$ populations. In terms of the density of CCPs distributed at the PMs, labelled either by CLC2GFP or TPLATE-GFP, the $g n^{s}$ mutants were remarkably normal (Figure 3C, 3E, and Video 12-15). When life times of the individual CLC2-GFP and TPLATE-GFP foci at the PMs, indicative of the temporal characteristics of the endocytic vesicle formation events, were measured from kymographs of TIRF time lapses, the distribution of TPLATE-GFP foci life times in $g n^{s}$ mutants was very similar to wild type controls (Figure $3 F$ ), while the distribution of CLC2-GFP foci life times varied slightly, showing a higher representation of short events (8-14 s) in the wild type, compared with $g n^{s}$ seedlings (Figure 3D). Yet, we do not consider this minor difference to be indicative of a defective CME in gn null mutants: Should GN function rely on $\mathrm{CME}$, the strong deficiency in overall growth and development in $g n$ knockouts would correspond to a similarly major defect in CME.

In summary, the lack of co-localization of GN-specific structures at the PM with CCPs, as well as lack of significant deficiencies in CME in $g n$ knockout seedlings, argue that the function of $\mathrm{GN}$ at the PM is not directly linked to the endocytic process.

\section{Bulk secretion proceeds normally in the absence of $\mathrm{GN}$}


To complement the experiments scrutinizing the GN function in CME, we tested the function of the secretory pathway in $g n^{s}$ mutants. For this purpose we crossed $g n^{s}$ with secRFP, a line expressing a variant of red fluorescent protein (RFP) containing a signal peptide guiding it into the lumen of the endoplasmic reticulum (ER), ultimately causing the protein to be secreted to the apoplast. Using CLSM, we found that secRFP was normally secreted to the apoplast in basal ends of $g n^{s}$ seedlings (Figure $4 \mathrm{~A}$ ), and in other parts of the seedling (Figure 4B), suggesting a functional secretory pathway. secRFP secretion was also normal in 2 day old wild type seedlings with roots closer resembling $g n^{s}$ basal ends (Figure 4-figure supplement 1A). Apparent intracellular fluorescent signals in $g n^{s}$ basal ends represented autofluorescence, likely of plastids, as these signals were also observed in $g n^{s}$ not expressing secRFP(Figure 4-figure supplement 1B).

Taken together, both CME and bulk secretion function correctly in the $g n^{s}$ mutant. The function of GN in the endomembrane system, which translates into its role in auxinmediated developmental patterning, is thus rather associated with the control of specific targets, candidates for which are PINs, or unknown regulators of PIN polarity (Steinmann et al., 1999; Zhang et al., 2020); D6PK, or unknown regulators of its association with the PM (Barbosa et al., 2014); and pectin components of the cell wall or proteins regulating their deposition (Shevell et al., 2000; Wachsman et al., 2020). As a further exploration of GN's activity in the endomembrane system, in the following, we describe a GN-specific exocytic event, which is indicative of GN's selective association with unknown components of the endomembrane system, possibly regulators involved in its unique cellular activity that translates into developmental patterning.

\section{BFA-induced exocytosis of GN, but not GNL1, indicates specific molecular interactions}

It has been previously observed that GN association with the PM is increased following its inhibition with BFA (Naramoto et al., 2010). Through a re-assessment of this artificially induced phenomenon, we gained additional insights into the specificity of GN action in the endomembrane system in comparison with GNL1. We re-evaluated the observation using the previously generated $G N_{\text {pro: }}$ GN-GFP VAN3 ${ }_{\text {pro: }}$ VAN3-mRFP reporter line. We note that for an unknown reason, this GN-GFP fluorescent reporter appears to localize to the PM only variably when observed with CLSM in undisturbed conditions (Naramoto et al., 2010 and our observations), in contrast to the GN-GFP reporters generated in the present study, where a localization to the PM is consistent (Figure 1A).

In BFA-treated conditions, besides localizing to the PM (Figure 5A), GN-GFP was strongly associated with cell plates in dividing cells (Figure 5-figure supplement $1 \mathrm{~A}$ ). The presence at cell plates indicated that the localization pattern of GN following BFA treatments 
may represent the outcome of an anterograde secretory movement of GN through the endomembrane system: this pathway leads to the PM in interphase cells, but preferentially to the cell plate during cell division (Richter et al., 2014). An anterograde traffic of BFAinhibited would be consistent with the previous observation that BFA-inhibited GN relocates from the GA to the TGN compartments (Naramoto et al., 2014). This can be perceived as a first step in an anterograde translocation of BFA-inhibited GN from the GA, through the TGN, and ultimately to the PM or the cell plate by exocytosis. As a cytosolic protein peripherally binding with membranes, we presume GN to translocate through the secretory pathway while continuously bound to membranes of organelles and secretory vesicles, likely due to its chemically inhibited state.

To test the notion that the localization of the BFA-inhibited GN at the PM represents

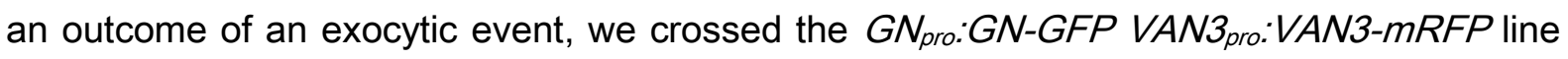
with big3, a mutant lacking the only BFA-resistant homologue within the BIG ARF-GEF family required for exocytosis and cell plate-directed transport from the TGN (Richter et al., 2014; Xue et al., 2019). A BFA treatment in this line causes a block of exocytosis from the TGN due to the inhibition of the remaining, BFA-sensitive ARF-GEFs of the BIG class. If the relocation of $\mathrm{GN}$ to the $\mathrm{PM}$ occurs through exocytosis, it is expected to be inhibited in this mutant background. Indeed, following a treatment with BFA at $50 \mu \mathrm{M}$ for $1 \mathrm{~h}$ in big3, the relocation of GN-GFP to the PM was minimal (Figure 5B and 5E). As an independent verification, we introduced into $G N_{\text {pro: }}$ GN-GFP VAN3 ${ }_{\text {pro: }}$ VAN3-mRFP an estradiol-inducible artificial microRNA construct down-regulating the expression of CLATHRIN HEAVY CHAIN $(\mathrm{CHC})$ genes, which encode a key structural component of the clathrin coats. Following silencing of $\mathrm{CHC}$, secretion is inhibited and secretory cargoes are re-routed to the vacuole (Adamowski et al., 2021). In XVE»amiCHCa, the BFA-induced relocation of GN-GFP to the $\mathrm{PM}$ too, was inhibited (Figure 5B, 5F, and Figure 5-figure supplement $1 \mathrm{~B}$ ). This finding further supports our conclusion that BFA-inhibited GN undergoes relocation to the PM on exocytic vesicles.

Next, we analyzed the BFA-induced localization patterns of the GN fwr variant. Interestingly, GN fwr_GFP, which in native conditions is present at the PM, but not the GA (Figure 2), became associated with the TGN, now at the core of the BFA bodies, but did not relocate to the PM (Figure $5 \mathrm{C}$ and $5 \mathrm{E}$ ). As such, it appears that the fwr mutation affects not only the normal recruitment of GN to the GA, but also its association with the BFA-induced exocytic process. Additionally, GNiwr is presumably de novo recruited to the TGN following its inhibition with BFA. The exact mechanism of these phenomena is currently unclear.

To address the specificity of the induced exocytosis of GN, we tested whether GNL1 undergoes a similar BFA-induced relocation. GNL1 is resistant to BFA due to a natural 
variation in the sequence of its catalytic SEC7 domain (Richter et al., 2007). As expected, the native, BFA-resistant GNL1-GFP was retained at the GA, being distributed at the periphery of BFA bodies following a BFA treatment (Figure 5-figure supplement $1 \mathrm{C}$ ). For a meaningful comparison with $\mathrm{GN}$, we analysed the reactions of the engineered BFA-sensitive GNL1 variant, GNL1 1sens-YFP (Richter et al., 2007). When assayed in gn/1 GNL1 pro:GNL 1sens YFP $\times$ Col-0 F1 seedlings to provide the native, BFA-resistant GNL1 function, GNL1 sens_YFP underwent only a very limited relocation to the PM following a BFA treatment (Figure 5D and $5 \mathrm{E})$, indicating a selectivity of this process to GN. On the other hand, GNL1 ${ }^{\text {sens-YFP secreted }}$ to the PM strongly in homozygous gn/1 mutant background (Figure 5-figure supplement 1D). This, however, was a manifestation of a non-specific disruption of the endomembrane system in the sensitized $g n / 1$ background, since a similar BFA-induced PM relocation of the TGN-localized VACUOLAR H+ ATPASE a1 subunit (VHAa1; Dettmer et al., 2006) could be observed in $g n / 1$, but not in the wild type (Figure 5-figure supplement 1E). The limited PM relocation of GNL1sens-YFP, as compared with GN-GFP, when compared in wild type backgrounds, demonstrates the preferential association of GN to its specific, BFA-induced exocytic pathway.

In summary, when inhibited by BFA, GN undergoes a specific exocytic relocation to the PM, likely bound to the surface of vesicles. GNL1 has a very limited affinity to this pathway. This process is distinct from a general relocation of endomembrane components caused by BFA in sensitized genetic backgrounds. We recognize this exocytic event only as a phenomenon induced by BFA, rather than representing a part of a normal mechanism of GN function. That said, the importance of the observation lies in the notion that BFAinhibited GN may be recruited to the presumed exocytic vesicles by molecular interactions which distinguish it from GNL1. Considering the difference in GN and GNL1 function, the experiments indicate presumed molecular components of GN's activity in developmental patterning within the endomembrane system.

\section{GN-GNL1 chimeras suggest functionally overlapping GN-specific features in all GN domains}

The comparison of GN and GNL1, presented above, uncovers a localization of GN to unknown structures at the PM, and a BFA-induced association of GN with unknown molecular components present on secretory vesicles. Both these phenomena are specific to GN when compared with its homologue GNL1. We investigated where in the GN protein are found sequences giving $\mathrm{GN}$ its distinct characteristics, manifested by these cell biological phenomena, and ultimately, responsible for its unique function in developmental patterning.

The large ARF-GEFs of GBF1 class, as well as the related ARF-GEFs of BIG class, are composed of a similar set of domains. At the $\mathrm{N}$-terminus are present the DCB 
(dimerization and cyclophilin binding) and HUS (homology upstream of SEC7) domains, followed by the SEC7 domain, and finally by HDS (homology downstream of SEC7) domains at the C-terminus, of which there are 3 in the GBF1 class and 4 in BIG class (Wright et al., 2014). The SEC7 domain engages directly with an ARF substrate and catalyses the exchange of GDP to GTP (Mossessova et al., 1998; Cherfils et al., 1998), leading to membrane docking and activation of the ARF. The functions of the non-catalytic DCB, HUS, and HDS domains are partially characterized. Studies of BIG and GBF1 class ARF-GEFs show that these domains function in membrane association of the large ARF-GEFs, and in allosteric regulations of the catalytic activity of the SEC7 domain (Richardson et al., 2012, 2016; Galindo et al., 2016; McDonold and Fromme, 2014; Halaby and Fromme, 2018; Gustafson and Fromme, 2017; Nawrotek et al., 2016; Meissner et al., 2018). These functions often depend on interactions of the non-catalytic domains with membranes, either direct, or through binding to activated ARF, ARF-LIKE, and Rab GTPases. In this sense, ARF-GEFs are not only activators, but also effectors of small GTPases, creating positive feedback loops, and cascades, in small GTPase networks (Stalder and Antonny, 2013; Richardson and Fromme, 2012; Lowery et al., 2013).

The difference in the molecular functions of GN and GNL1, ultimately expressed in their functions on the organismal scale, and evidenced by a lack of gn mutant rescue by GNL1 expression, by the association of GN to the PM-localized structures, and by the preferential association of GN to the BFA-induced secretory process, must be, in the most basic sense, reflected in the differences in the amino acid sequences of these two ARFGEFs. To identify the determinants of this special GN function within the GN protein sequence, we cloned a series of chimeric GBF1 class ARF-GEFs composed of domains originated from GN and GNL1 proteins, and tested their activity in the molecular function of GN. We divided the ARF-GEFs into three components: (i) the N-terminal regulatory DCBHUS domains, (ii) the catalytic SEC7 domain, and (iii) C-terminal regulatory HDS1-3 domains. While the three HDS domains are likely not identical in activities, at this stage we analyzed their contribution as a single entity. The resulting chimeras are named by threeletter acronyms indicating the subsequent components of $G N(G)$ and GNL1 (L) origin (Figure 6A). Of the possible chimeras, we generated all except LLG. Constructs expressing GFP fusions of the chimeras, controlled by the GN promoter, were transformed into heterozygous $g n^{s}$ plants to test their ability to replace the function of GN. Homozygous rescued mutants were isolated where possible; otherwise segregating $g n^{s}$ progenies were analysed. All phenotypes described in the following were observed in two independent transgenic lines for each chimera (Figure 7-figure supplement 2). $g n^{s} G N_{\text {pro: }}$ GN-GFP and $g n^{s}$ 
GN pro:GNL1-GFP lines (Figure 1-Figure supplement 1) constitute controls for this experiment. The summary of all described phenotypes is given in Table 1.

First, we consider in isolation the functions of DCB/HUS and HDS domains. The LGG chimera, consisting of the DCB/HUS domain of GNL1 and the remaining domains of GN, was fully functional, as reflected by normal phenotypes of the complemented mutants both at seedling and adult stages (Figure 6C, 7A, and 7B). Root hair positioning at basal cell sides was also normal (Figure 1-figure supplement 1D). Conversely, a GLL chimera, where only the DCB/HUS domain originates from GN, did not exhibit any GN function, as $g n^{s}$ seedlings expressing this chimera retained the typical gn phenotypes (Figure 6B). These observations indicate that differences between the DCB/HUS domains of GN and its homologue GNL1 are not responsible for the specific molecular action of GN.

Next, we consider the HDS domains. The GGL chimera, where the HDS domains of GN are replaced with GNL1, was almost fully functional: While the adult plants developed normally (Figure 6C), the seedlings, which were of normal size, exhibited a slight alteration in root growth pattern, characterized by a visibly more straight growth (Figure 7A). When grown in Petri dishes tilted back from the vertical, which in the wild type induces a wavy pattern of root growth, this straight growth was well expressed by the partial loss of root waving (Figure 7B). This phenotype may be caused by a slight defect in polar auxin transport, since low doses of auxin transport inhibitor N-1-naphthylphthalamic acid (NPA) produce similar effects (Figure 7-figure supplement 1). Overall, however, the phenotype of $g n^{s}$ complemented by the expression of GGL indicates only a minor contribution of the HDS sequences specific to GN, compared with those present in GNL1, to the function of GN.

Interestingly, however, when the DCB/HUS and the HDS domains of GN were simultaneously replaced by their counterparts from GNL1 in the LGL chimera, the GNspecific function was significantly affected. The adult $g n^{s} G N_{\text {pro: }}$ LGL-GFP plants developed very slowly, and were characterized by small and compact rosettes of leaves. The individuals which did bolt, grew short stems with floral development arrested at an early stage (Figure 6D). Left to grow for several weeks after their wild type siblings completed development, the plants grew numerous stems which remained short (Figure 6-figure supplement 1A). Ultimately, the plants were completely infertile, and the lines were maintained as $g n^{s+/-}$. Seedlings of $g n^{s} G N_{\text {pro: }}: L$ GL-GFP developed very short roots (Figure 7C), did not exhibit any root waving (Figure 7D), and in up to $50 \%$ of cases possessed single, or partially fused, cotyledons (Figure 7C and 7E), an embryonic defect typical to gn mutants (Shevell et al., 1994; Geldner et al., 2004). Dark-grown seedlings were clearly agravitropic (Figure 7F and Figure 6-figure supplement 1C) and their apical hooks were open at $75 \mathrm{~h}$ of in vitro growth (Figure $7 \mathrm{G}$ ). These various deficiencies demonstrate that the 
DCB/HUS and HDS domains of GN, compared with their counterparts in GNL1, do, in fact, possess features required for the specific molecular function of GN. The observation that the function of GN becomes defective as a result of their simultaneous absence, but not when absent individually, suggests that the molecular function of GN may be mediated through multiple and partially redundant molecular interactions in which these regulatory domains are involved.

Finally, we analysed the contribution of the catalytic SEC7 domain of GN. $g n^{s}$ complemented by GLG, a chimera where the SEC7 domain of GN is replaced by its counterpart from GNL1, provided a partial, but significant, level of function. Adult plants developed clearly better than plants expressing LGL, but visibly weaker than plants expressing GN, GGL, or LGG. Compared with the latter, they were characterized by smaller rosettes, and by the presence of fewer stems, which, however, were long (Figure 6C and Figure 6-figure supplement $1 \mathrm{~B}$ ). The fertility of $g n^{s} G N_{\text {pro: }}$ GLG-GFP plants was limited. A comparison of seedling phenotypes between $g n^{s}$ complemented by GLG and by LGL was the most salient observation in our study of GN-GNL1 chimeras. These two chimeras express mutually exclusive domains of $\mathrm{GN}$, and yet, the phenotypes resulting from $g n^{s}$ complementation were, in the qualitative sense, the same. Specifically, similarly to $g n^{s}$

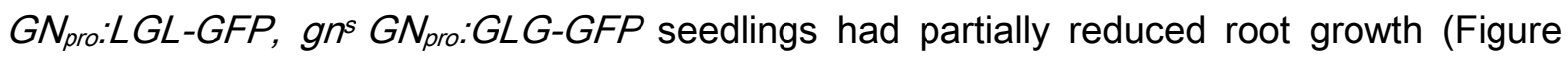
7C), a deficiency in the root waving response (Figure 7D), exhibited up to $50 \%$ of seedlings with partially or completely fused cotyledons (Figure 7C and 7E), had agravitropic etiolated seedlings (Figure 7F and Figure 6-figure supplement $1 \mathrm{C}$ ), and open apical hooks at $75 \mathrm{~h}$ of development in in vitro cultures (Figure 7G).

The comparison of chimeras with mutually exclusive domain composition shows clearly that the unique molecular function of GN cannot be ascribed to any single domain of the GN protein. It is likely that multiple interactions of GN DCB/HUS, SEC7, and HDS domains, in allosteric regulations within single GN proteins as well as between GN domains and their unknown external interactors, including small GTPases, may all contribute, in a somewhat quantitative and partly redundant sense, to the specific molecular function of GN, distinguishing this ARF-GEF from GNL1.

\section{Subcellular localization and BFA-induced exocytosis of GN-GNL1 chimeras}

To complete the analysis of the GN-GNL1 chimeras, we assessed them in the context of the GN activity at the newly identified structures at the PM (Figure 1), and of the GN-specific BFA-induced exocytic process (Figure 5). When etiolated hypocotyls of $g n^{s}$ complemented by any of the functional chimeras, that is, LGG-GFP, GGL-GFP, GLG-GFP, and LGL-GFP, were analyzed by TIRF microscopy, all these chimeric ARF-GEFs localized to the GA as 
well as in the punctate structures at the PM, identical in appearance to those where GN-GFP localizes (Figure 8A and Figure 8-figure supplement 1A). The non-functional chimera GLLGFP, expressed in the wild type, could be seen at the GA and relatively sparsely at GNspecific PM structures, as well (Figure 8B). The recruitment of the chimeric ARF-GEFs to these structures at the PM, mediated through any domain of GN, supports our conclusion of their role in the specific developmental function of GN, but the localization of GLL-GFP indicates that localization alone may not be sufficient to carry out GN function.

Next, we tested whether chimeric ARF-GEFs are recruited into the GN-specific, BFAinduced exocytic pathway. We used chimera-GFP reporters expressed in wild type background, rather than the variably rescued mutants, to obtain more directly comparable results. In these lines, the chimeras were not reliably observed by CLSM at the PMs of seedling RAMs in undisturbed conditions. This may be due to low signal intensity at the PM relative to the cytosolic and GA-localized signals, possibly resulting from competition with the native GN, which presumably recruits to the PM sites of action more effectively. Overall, in the course of experiments presented in this study, the localization of GN to the PM was most reliably observed with TIRF microscopy, rather than by CLSM; somewhat analogically, CCPS are always observed at the PM with TIRF microscopy, but the detection of fluorescent protein fusions of CLC at the PMs of RAM epidermis by CLSM is limited (e.g. Adamowski et al., 2018, 2021). Predictably, due to their resistance to BFA, chimeras with the SEC7 domain of GNL1, GLL-GFP and GLG-GFP, remained at the BFA body periphery where GA are found, and did not undergo BFA-induced relocations (Figure 8-figure supplement 1B), similar to the native, BFA-resistant GNL1 (Figure 5-figure supplement $1 \mathrm{C}$ ). Thus, a comparison of BFA-induced relocation to the PM was conducted between GN-GFP, LGG-GFP, GGL-GFP, and LGL-GFP. We found that the chimeras with relatively larger portions of the GN protein, that is, LGG-GFP and GGL-GFP, significantly relocated to the PM following BFA treatments, although to a degree measurably lower than GN-GFP (Figure $8 \mathrm{C}$ and Figure 8-figure supplement $1 \mathrm{C}$ ). In turn, the chimera with a smallest contribution of GN sequence, LGLGFP, did not exhibit any relocation, and likely remained at the GA (Figure 8C and Figure 8figure supplement $1 \mathrm{C}$ ). These observations further show the selective affinity of $\mathrm{GN}$ to the BFA-induced exocytic process, and reflect the manner in which the domains of GN quantitatively contribute to the molecular character of this ARF-GEF, as identified based on morphological phenotypes.

\section{Discussion}

The mechanism behind the unique developmental function of GN ARF-GEF has been a subject of intense research. The information obtained over the last decades significantly 
contributed to our understanding of how the function of the endomembrane system impacts on patterning and polarity of the whole plant body. In this study, we provide novel insights into this function, taking into consideration the molecular nature of GN as an ARF-GEF component acting in the endomembrane system, and taking advantage of the unique situation where GNL1, its close homologue in terms of sequence and structure, exhibits distinct cellular and developmental functions. With the use of GN-GNL1 chimeras, we attempted to identify specific domains of the GN protein responsible for its unique function. This analysis indicated that all GN domains: the regulatory DCB/HUS and HDS domains as well as the catalytic SEC7 domain, contain unique sequences that contribute, in a somewhat redundant fashion, to placing $\mathrm{GN}$ in a specific molecular environment within the cell. The hypothetical molecular associations collectively mediated by these GN-specific domains also promote a selective affinity of GN to the exocytic machinery, induced by GN inhibition with $B F A$. This artificially induced process could be observed due to the coincidental combination of BFA-resistant and BFA-sensitive ARF-GEF isoforms in the $A$. thaliana model system. Were GNL1 BFA-sensitive, the specific secretion of GN could not be observed due to a general endomembrane system breakdown, and were BIG3 BFA-sensitive, no GN-specific exocytosis could occur. Such realizations highlight the caution required when interpreting observations resulting from chemical inhibition.

One central proposition of our work is that the developmental function of $\mathrm{GN}$ is mediated from the PM, from rather stable structures of an unknown nature. This interpretation is based on the observation that the localization to the PM distinguishes functional GN-GFP reporters, as well as GN-GNL1 chimeras, from GNL1, and crucially, that GN fwr, a mutant variant of GN exhibiting a complete or almost complete function, localizes exclusively to these structures but cannot be detected at the other site of GN action, the GA. In our view, the localization of $G N$ to the GA represents only its contribution to the fundamental secretory activity, an ancestral role which it shares with GNL1 (Richter et al., 2007). The ARF-GAP VAN3, which exhibits a function in auxin-mediated developmental patterning like GN, acts from the PM as well (Naramoto and Kyozuka, 2018), but localizes there to dense and very dynamic structures distinct from those that recruit GN. As is the case of GN, the function of VAN3 at the PM is not directly connected to CME (Adamowski and Friml, 2021).

A direct action of $\mathrm{GN}$ at the PM appears consistent with the rapid and sensitive manner in which its inhibition by BFA causes a loss of D6PK from its polar PM domain (Barbosa et al., 2014). With regard to the mechanism by which GN controls the polar distribution of PIN auxin transporters, our findings argue against a model where GN promotes an ARF-dependent formation of exocytic vesicles trafficking PINs from an 
intracellular compartment to the polar domain at the PM. Considering not only its site of action at the PM required for developmental patterning, but also the reported non-cell autonomous effect of GN on PIN polarization (Wolters et al., 2011), we favour an indirect model of GN action on PIN polarity, where its activity influences other, unknown polarity components, which in turn instruct the vesicular sorting of PIN. It is possible that the polarity determinants regulated by GN at the PM are in some way associated with the cell wall. This is hinted not only by the alteration of cell wall structure in gn mutants (Shevell et al., 2000; Wachsman et al., 2020), but also by the identification of a cellulose synthase mutant in the regulator of pin polarity (repp) forward genetic screen, based on an abnormal polar sorting of ectopically expressed PIN1 to the apical, rather than the basal, polar domain of epidermal cells (Feraru et al., 2011). It could be conceived that GN-controlled polarity determinants within the cell wall, and/or associated with it from the PM, provide information orienting not only the polar domains of individual cells, but also, as an apoplastic continuum, coordinating tissue-wide polarity patterns in the symplast (Steinmann et al., 1999), enabling patterned development of tissues, prominently vasculature (Verna et al., 2019), and of the body plan as a whole.

\section{Materials and methods}

\begin{tabular}{|c|c|c|c|c|}
\hline \multicolumn{5}{|l|}{ Key Resources Table } \\
\hline $\begin{array}{l}\text { Reagent type } \\
\text { (species) or } \\
\text { resource }\end{array}$ & Designation & $\begin{array}{l}\text { Source or } \\
\text { reference }\end{array}$ & Identifiers & $\begin{array}{l}\text { Additional } \\
\text { information }\end{array}$ \\
\hline \multicolumn{5}{|l|}{$\begin{array}{l}\text { Biological sample } \\
\text { (Arabidopsis } \\
\text { thaliana, Col-0) }\end{array}$} \\
\hline $\begin{array}{l}\text { Gene (Arabidopsis } \\
\text { thaliana) }\end{array}$ & GNOM & $\begin{array}{l}\text { The Arabidopsis } \\
\text { Information } \\
\text { Resource }\end{array}$ & AT1G13980 & \\
\hline $\begin{array}{l}\text { Gene (Arabidopsis } \\
\text { thaliana) }\end{array}$ & $\begin{array}{l}\text { GNOM- } \\
\text { LIKE1 }\end{array}$ & $\begin{array}{l}\text { The Arabidopsis } \\
\text { Information } \\
\text { Resource }\end{array}$ & AT5G39500 & \\
\hline $\begin{array}{l}\text { Genetic reagent } \\
\text { (Arabidopsis }\end{array}$ & $\mathrm{gn}^{\mathrm{s}}$ & $\begin{array}{l}\text { SALK T-DNA } \\
\text { collection }\end{array}$ & SALK_103014 & \\
\hline
\end{tabular}




\begin{tabular}{|l|l|l|l|l|}
\hline thaliana) & & & & \\
\hline
\end{tabular}

\section{Plant material}

The following previously described $A$. thaliana lines were used in this study: $g n^{s}$

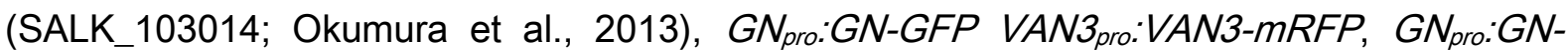
GFP 35S pro:CLC-mKO (Naramoto et al., 2010), CLC2 pro:CLC2-GFP (Konopka et al., 2008), LAT52 pro:TPLATE-GFP RPS5A ID N799370; Samalova et al. 2006), big3 (SALK_044617; Richter et al., 2014), XVE»amiCHCa (Adamowski et al., 2021), gn/1 GNL 1 pro:GNL 1sens_ YFP (Richter et al., 2007),

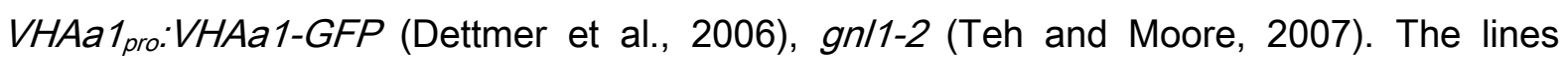
generated as part of this study as listed in Supplementary File 1a.

\section{In vitro cultures of Arabidopsis seedlings}

Seedlings were grown in in vitro cultures on half-strength Murashige and Skoog $(1 / 2 \mathrm{MS})$ medium of $\mathrm{pH}=5.9$ supplemented with $1 \%(\mathrm{w} / \mathrm{v})$ sucrose and $0.8 \%(\mathrm{w} / \mathrm{v})$ phytoagar at $21^{\circ} \mathrm{C}$ in 16h light/8h dark cycles with Philips GreenPower LED as light source, using deep red $(660 \mathrm{~nm}) / \mathrm{far}$ red $(720 \mathrm{~nm}) / \mathrm{blue}(455 \mathrm{~nm})$ combination, with a photon density of about $140 \mu \mathrm{mol} /\left(\mathrm{m}^{2} \mathrm{~s}\right)+/-20 \%$. Petri dishes for TIRF imaging in hypocotyls of etiolated seedlings, and for studies of development of etiolated seedlings, were initially exposed to light for several hours and then wrapped in aluminium foil.

\section{Chemical treatments}

BFA (Sigma-Aldrich B7651) was solubilized in DMSO to $50 \mathrm{mM}$ stock concentration and added to liquid $1 / 2 \mathrm{MS}$ media for treatments. Beta-estradiol (Sigma-Aldrich E8875) was solubilized in $100 \%$ ethanol to $5 \mathrm{mg} / \mathrm{mL}$ stock concentration and added to $1 / 2 \mathrm{MS}$ media during preparation of solid media to a final concentration of $2.5 \mu \mathrm{g} / \mathrm{mL}$. Induction of XVE»amiCHCa was performed approximately $48 \mathrm{~h}$ before CLSM imaging by transferring 3 day old seedlings to media supplemented with beta-estradiol.

\section{Molecular cloning and generation of transgenic lines}

All constructs were generated using the Gateway method (Invitrogen) and are listed in Supplementary File 1c. DNA sequences were amplified by PCR reactions using iProof High Fidelity polymerase (BioRad). Primers used for cloning are listed in Supplementary File $1 \mathrm{~b}$. GN-GNL 1 chimeras were cloned using overlap extension PCR (fusion PCR) from cDNA templates. Fragment 1 (DCB-HUS) corresponds to nucleotides 1-1653 in both ARF-GEFs, fragment 2 (SEC7) to nucleotides 1654-2268 in both ARF-GEFs, and fragment 3 (HDS1-3) 
to nucleotides 2269-4329 and 2269-4353 in GN and GNL1, respectively. GN, GNL1 and chimeric GGL, GLG, LGG, GLL, LGL sequences without stop codons were introduced by Gateway BP Clonase into pDONR221 vectors. GN promoter region (nucleotides -2127 to -1 ; Geldner et al., 2003) was PCR-amplified and introduced by Gateway BP Clonase into pDONRP4P1r vector. Expression vectors were made by combining $G N_{\text {pro }}$, ARF-GEF coding sequences, and GFP/pDONRP2rP3, in pH7m34GW expression vector (Karimi et al., 2002) using Gateway LR Clonase II Plus. $g n^{s+/-}$ were transformed by the standard floral dip method, and T1 plants were selected on $1 / 2 \mathrm{MS}$ medium without sucrose supplemented with hygromycin. T1 plants were genotyped for $g n^{s}$ insertion using primers gn-SALK-F and gnSALK-R, of which gn-SALK-R binds to the $G N$ intron absent in the transgenes. FWR mutation was introduced using overlap extension PCR (fusion PCR) from GN cDNA template by substitution of ${ }^{3013}$ TCT for ${ }^{3013}$ TTT. The rest of the procedure was analogical to that described above.

\section{Light microscopy}

High magnification images of seedlings developing in in vitro cultures were taken with Leica EZ4 HD stereomicroscope equipped with $0.8-3.5 x$ magnification lens, by an integrated 3 megapixel CMOS camera.

\section{Root hair positioning}

Relative root hair positions were measured on light microscopic images using Fiji (https://imagej.net/Fiji). Distances of root hair outgrowths from apical (a) and basal (b) cell ends were measured and relative position calculated as $b /(a+b)$.

\section{Confocal Laser Scanning Microscopy}

4 to $5 \mathrm{~d}$ old seedlings were used for live imaging with Zeiss 800 confocal laser scanning microscope with $10 \times 0.45$ air, 20X 0.8 air, and 40X 1.2 water lenses. Excitation wavelengths $488 \mathrm{~nm}$ and $561 \mathrm{~nm}$. Detector type: two gallium arsenide phosphide photomultiplier tube detectors (GaAsP PMTs) with free choice of spectral range (emission ranges were selected to optimize detection while reducing background autofluorescence). Gain was set according to fluorescent protein expression levels in the range 650-800 V. Line averaging $2 \mathrm{X}$, offset 0 . Z-stacks were captured with 0.6-1 $\mu \mathrm{m}$ spacing. Relative PM signal intensities of ARF-GEFGFP fusions following BFA treatments were measured using Fiji (https://imagej.net/Fiji) as a ratio between mean grey values of a line of 5 pixel width drawn over multiple PMs, and of a rectangle covering the whole RAM surface visible in a CLSM image. Sample sizes (given in figure legends) and number of repetitions (2-4) were decided through experimenter's experience. 


\section{Total Internal Reflection Fluorescence microscopy}

Early elongation zone of roots in excised $\sim 1 \mathrm{~cm}$ long root tip fragments from $7 \mathrm{~d}$ old seedlings, as well as 3 to $5 \mathrm{~d}$ old etiolated wild type hypocotyls and $g n$ mutant seedlings, were used for TIRF imaging. Imaging was performed with Olympus IX83 TIRF microscope, using a 100X 1.40 oil UAPON OTIRF lens with an additional 1.6X magnification lens in the optical path. Excitation wavelengths $488 \mathrm{~nm}$ and $561 \mathrm{~nm}$. Due to the nature of imaged tissues, laser illumination angle was adjusted individually to each sample to optimize detection of signals near cell surface with minimal background. Detector type: Hammamatsu ImagEM X2 EM-CCD C9100-13. EM gain was set in the range 200-400V depending on fluorescent reporter expression levels. For single channel GFP imaging, a dichroic LM491 filter and a 525/45 bandpass filters were used. For two channel imaging, a quad line beamsplitter ZT405/488/561/640rpc (Chroma) was employed (reflected wavelengths 400$410 \mathrm{~nm}, 485-493 \mathrm{~nm}, 555-564 \mathrm{~nm}, 630-644 \mathrm{~nm}$ ). Time lapses of 100 frames at $1 \mathrm{~s}$ intervals with exposure times of $200 \mathrm{~ms}$, or single snapshots of $200 \mathrm{~ms}$ exposure, were taken, depending on the experiment. Two-channel imaging was performed sequentially with 200 ms exposure times. Quantitative evaluations were performed using Fiji (https://imagej.net/Fiji). CLC2-GFP and TPLATE-GFP foci were counted in square regions of $36 \mu \mathrm{m}^{2}$ taken from the captured TIRF images or movies. Lifetimes of CLC2-GFP and TPLATE-GFP were measured in kymographs extracted from each captured TIRF movie. GN-GFP foci were counted in square regions of $110.25 \mu \mathrm{m}^{2}$ taken from the captured TIRF images or movies. Lateral displacement of GN-GFP signals over time was measured on maximum projections of kymographs. Sample sizes (given in figure legends) and number of repetitions (2-4) were decided through experimenter's experience.

\section{Accession numbers}

Sequence data from this article can be found in the GenBank/EMBL libraries under the following accession numbers: GNOM (AT1G13980), GNOM-LIKE1 (AT5G39500), VAN3 (AT5G13300), BIG3 (AT1G01960), CLC2 (AT2G40060), TPLATE (AT3G01780), VHAa1 (AT2G28520).

\section{Acknowledgements}

The authors would like to gratefully acknowledge $\mathrm{Dr}$ Xixi Zhang for cloning the GNL 1/pDONR221 construct and for useful discussions.

\section{References}

Adamowski, M. and Friml, J. (2015). PIN-dependent auxin transport: Action, regulation, and evolution. Plant Cell 27. 
Adamowski, M., and Friml, J. (2021) Clathrin-mediated endocytosis-independent function of VAN3 ARF GTPase Activating Protein at the plasma membrane BIORXIV/2021/468981

Adamowski, M., Matijević, I., and Friml, J. (2021) The role of clathrin in exocytosis and the mutual regulation of endo- and exocytosis in plant cells. BIORXIV/2021/468992

Barbosa, I.C.R., Zourelidou, M., Willige, B.C., Weller, B., and Schwechheimer, C. (2014). D6 PROTEIN KINASE activates auxin transport-dependent growth and PIN-FORMED phosphorylation atthe plasma membrane. Dev. Cell 29:674-685.

Cherfils, J., Ménétrey, J., Mathieu, M., Le Bras, G., Robineau, S., Béraud-Dufour, S., Antonny, B., and Chardin, P. (1998). Structure of the Sec7 domain of the Arf exchange factor ARNO. Nature 392: 101-105.

Dettmer, J., Hong-Hermesdorf, A., Stierhof, Y.-D., and Schumacher, K. (2006). Vacuolar H+ -ATPase Activity Is Required for Endocytic and Secretory Trafficking in Arabidopsis. Plant Cell 18: 715-730.

Deyholos, M.K., Cordner, G., Beebe, D., and Sieburth, L.E. (2000). The SCARFACE gene is required for cotyledon and leaf vein patterning. Development 3213: 3205-3213.

Donaldson, J.G. and Jackson, C.L. (2011). ARF family G proteins and their regulators: roles in membrane transport, development and disease. Nat. Rev. Mol. Cell Biol. 12.

Feraru, E., Feraru, M.I., Kleine-Vehn, J., Martinière, A., Mouille, G., Vanneste, S., Vernhettes, S., Runions, J., and Friml, J. (2011). PIN Polarity Maintenance by the Cell Wall in Arabidopsis. Current Biology 21: 338-343.

Fischer, U., et al. (2006) Vectorial Information for Arabidopsis Planar Polarity Is Mediated by Combined AUX1, EIN2, and GNOM Activity. Current Biology, 16:2143-49.

Gadeyne, A. et al. (2014). The TPLATE adaptor complex drives clathrin-mediated endocytosis in plants. Cell 156: 691-704.

Galindo, A., Soler, N., McLaughlin, S.H., Yu, M., Williams, R.L., and Munro, S. (2016). Structural Insights into Arl1-Mediated Targeting of the Arf-GEF BIG1 to the trans-Golgi. Cell Reports 16: 839-850.

Geldner, N., Friml, J., Stierhof, Y.D., Jürgens, G., and Palme, K. (2001). Auxin transport inhibitors block PIN1 cycling and vesicle trafficking. Nature 413:425-428.

Geldner, N., Anders, N., Wolters, H., Keicher, J., Kornberger, W., Muller, P., Delbarre, A., Ueda, T., Nakano, A., and Jürgens, G. (2003). The Arabidopsis GNOM ARF-GEF Mediates Endosomal Recycling, Auxin Transport, and Auxin-Dependent Plant Growth. Cell 112: 219-230.

Geldner, N., Richter, S., Vieten, A., Marquardt, S., Torres-Ruiz, R. A., Mayer, U., and Jürgens, G. (2004). Partial loss-of-function alleles reveal a role for GNOM in auxin transport-related, post-embryonic development of Arabidopsis. Development 131: 389- 
400.

Gustafson, M.A. and Fromme, J.C. (2017). Regulation of Arf activation occurs via distinct mechanisms at early and late Golgi compartments. MBoC 28: 3660-3671.

Halaby, S.L. and Fromme, J.C. (2018). The HUS box is required for allosteric regulation of the Sec7 Arf-GEF. Journal of Biological Chemistry 293: 6682-6691.

Jackson, C.L. and Bouvet, S. (2014). Arfs at a Glance. J. Cell Sci. 127: 4103-4109.

Jelínková, A., Malínská, K., Simon, S., Kleine-Vehn, J., Pařezová, M., Pejchar, P., Kubeš, M., Martinec, J., Friml, J., Zažímalová, E., and Petrášek, J. (2010). Probing plant membranes with FM dyes: tracking, dragging or blocking? The Plant Journal 61: 883892.

Karimi, M., Inzé, D., and Depicker, A. (2002). GATEWAYTM vectors for Agrobacteriummediated plant transformation. Trends Plant Sci. 7: 193-195.

Kleine-Vehn, J., Dhonukshe, P., Sauer, M., Brewer, P.B., Wiśniewska, J., Paciorek, T., Benková, E., and Friml, J. (2008). ARF GEF-Dependent Transcytosis and Polar Delivery of PIN Auxin Carriers in Arabidopsis. Current Biology 18: 526-531.

Kleine-Vehn, J., Ding, Z., Jones, A.R., Tasaka, M., Morita, M.T., and Friml, J. (2010). Gravity-induced PIN transcytosis for polarization of auxin fluxes in gravity-sensing root cells. Proceedings of the National Academy of Sciences 107: 22344-22349.

Koizumi, K., Naramoto, S., Sawa, S., Yahara, N., Ueda, T., Nakano, A., Sugiyama, M., and Fukuda, H. (2005). VAN3 ARF - GAP-mediated vesicle transport is involved in leaf vascular network formation. Development: 1699-1711.

Koizumi, K., Sugiyama, M., and Fukuda, H. (2000). A series of novel mutants of Arabidopsis thaliana that are defective in the formation of continuous vascular network: calling the auxin signal flow canalization hypothesis into question. Development 127: 3197-204.

Konopka, C.A., Backues, S.K., and Bednarek, S.Y. (2008). Dynamics of Arabidopsis Dynamin-Related Protein $1 \mathrm{C}$ and a Clathrin Light Chain at the Plasma Membrane. Plant Cell Online 20: 1363-1380.

Lowery, J., Szul, T., Styers, M., Holloway, Z., Oorschot, V., Klumperman, J., and Sztul, E. (2013). The Sec7 Guanine Nucleotide Exchange Factor GBF1 Regulates Membrane Recruitment of BIG1 and BIG2 Guanine Nucleotide Exchange Factors to the TransGolgi Network (TGN). Journal of Biological Chemistry 288: 11532-11545.

Mayer, U., Büttner, G., and Jürgens, G. (1993). Apical-basal pattern formation in the Arabidopsis embryo: studies on the role of the gnom gene. Development 117: 149-162. Mayer, U., Ruiz, R.A.T., Berleth, T., Miséra, S., and Jürgens, G. (1991). Mutations affecting body organization in the Arabidopsis embryo. Nature 353: 402-407.

McDonold, C.M. and Fromme, J.C. (2014). Four GTPases Differentially Regulate the Sec7 
Arf-GEF to Direct Traffic at the trans-Golgi Network. Developmental Cell 30: 759-767.

Meinke, D.W. (1985). Embryo-lethal mutants of Arabidopsis thaliana: analysis of mutants with a wide range of lethal phases. Theoret. Appl. Genetics 69-69: 543-552.

Meissner, J.M., Bhatt, J.M., Lee, E., Styers, M.L., Ivanova, A.A., Kahn, R.A., and Sztul, E. (2018). The ARF guanine nucleotide exchange factor GBF1 is targeted to Golgi membranes through a PIP-binding domain. J Cell Sci 131: jcs210245.

Min, M.K., Jang, M., Lee, M., Lee, J., Song, K., Lee, Y., Choi, K.Y., Robinson, D.G., and Hwang, I. (2013). Recruitment of Arf1-GDP to Golgi by Glo3p-Type ArfGAPs Is Crucial for Golgi Maintenance and Plant Growth. Plant Physiol. 161: 676-691.

Min, M.K., Kim, S.J., Miao, Y., Shin, J., Jiang, L., and Hwang, I. (2007). Overexpression of Arabidopsis AGD7 Causes Relocation of Golgi-Localized Proteins to the Endoplasmic Reticulum and Inhibits Protein Trafficking in Plant Cells. Plant Physiol. 143: 1601-1614.

Miyazawa, Y., Takahashi, A., Kobayashi, A., Kaneyasu, T., Fujii, N., and Takahashi, H. (2009). GNOM-Mediated Vesicular Trafficking Plays an Essential Role in Hydrotropism of Arabidopsis Roots. Plant Physiol. 149: 835-840.

Moriwaki, T., Miyazawa, Y., Fujii, N., and Takahashi, H. (2014). GNOM regulates root hydrotropism and phototropism independently of PIN-mediated auxin transport. Plant Science 215-216: 141-149.

Mossessova, E., Gulbis, J.M., and Goldberg, J. (1998). Structure of the Guanine Nucleotide Exchange Factor Sec7 Domain of Human Arno and Analysis of the Interaction with ARF GTPase. Cell 92: 415-423.

Naramoto, S., Kleine-Vehn, J., Robert, S., Fujimoto, M., Dainobu, T., Paciorek, T., Ueda, T., Nakano, A., Van Montagu, M.C.E., Fukuda, H., and Friml, J. (2010). ADP-ribosylation factor machinery mediates endocytosis in plant cells. Proc. Natl. Acad. Sci. 107: 21890-21895.

Naramoto, S. and Kyozuka, J. (2018). ARF GTPase machinery at the plasma membrane regulates auxin transport-mediated plant growth. Plant Biotechnol. 35: 155-159.

Naramoto, S., Otegui, M.S., Kutsuna, N., de Rycke, R., Dainobu, T., Karampelias, M., Fujimoto, M., Feraru, E., Miki, D., Fukuda, H., Nakano, A., and Friml, J. (2014). Insights into the Localization and Function of the Membrane Trafficking Regulator GNOM ARFGEF at the Golgi Apparatus in Arabidopsis. Plant Cell 26: 3062-3076.

Naramoto, S., Sawa, S., Koizumi, K., Uemura, T., and Ueda, T. (2009). Phosphoinositidedependent regulation of VAN3 ARF-GAP localization and activity essential for vascular tissue continuity in plants. Development 1538: 1529-1538.

Nawrotek, A., Zeghouf, M., and Cherfils, J. (2016). Allosteric regulation of Arf GTPases and their GEFs at the membrane interface. Small GTPases 7: 283-296. 
Okumura, K., Goh, T., Toyokura, K., Kasahara, H., Takebayashi, Y., Mimura, T., Kamiya, Y., and Fukaki, H. (2013). GNOM/FEWER ROOTS is Required for the Establishment of an Auxin Response Maximum for Arabidopsis Lateral Root Initiation. Plant and Cell Physiology 54: 406-417.

Rakusová, H., Gallego-Bartolomé, J., Vanstraelen, M., Robert, H.S., Alabadí, D., Blázquez, M.A., Benková, E., and Friml, J. (2011). Polarization of PIN3-dependent auxin transport for hypocotyl gravitropic response in Arabidopsis thaliana: PIN3 polarization for hypocotyl gravitropism. The Plant Journal 67: 817-826.

Richardson, B.C. and Fromme, J.C. (2012). Autoregulation of Sec7 Arf-GEF activity and localization by positive feedback. Small GTPases 3: 240-243.

Richardson, B.C., Halaby, S.L., Gustafson, M.A., and Fromme, J.C. (2016). The Sec7 Nterminal regulatory domains facilitate membrane-proximal activation of the Arf1 GTPase. eLife 5: e12411.

Richardson, B.C., McDonold, C.M., and Fromme, J.C. (2012). The Sec7 Arf-GEF Is Recruited to the trans-Golgi Network by Positive Feedback. Developmental Cell 22: 799-810.

Richter, S., Anders, N., Wolters, H., Beckmann, H., Thomann, A., Heinrich, R., Schrader, J., Singh, M.K., Geldner, N., Mayer, U., and Jürgens, G. (2010). Role of the GNOM gene in Arabidopsis apical-basal patterning - From mutant phenotype to cellular mechanism of protein action. Eur. J. Cell Biol. 89: 138-144.

Richter, S., Geldner, N., Schrader, J., Wolters, H., Stierhof, Y.-D., Rios, G., Koncz, C., Robinson, D.G., and Jürgens, G. (2007). Functional diversification of closely related ARF-GEFs in protein secretion and recycling. Nature 448: 488-492.

Richter, S., Kientz, M., Brumm, S., Nielsen, M.E., Park, M., Gavidia, R., Krause, C., Voss, U., Beckmann, H., Mayer, U., Stierhof, Y.D., and Jürgens, G. (2014). Delivery of endocytosed proteins to the cell-division plane requires change of pathway from recycling to secretion. Elife 2014: 1-16.

Robineau, S., Chabre, M., and Antonny, B. (2000). Binding site of brefeldin A at the interface between the small $G$ protein ADP-ribosylation factor 1 (ARF1) and the nucleotideexchange factor Sec7 domain. Proceedings of the National Academy of Sciences 97: 9913-9918.

Robinson, D.G., Langhans, M., Saint-Jore-Dupas, C., and Hawes, C. (2008). BFA effects are tissue and not just plant specific. Trends in Plant Science 13: 405-408.

Samalova, M., Fricker, M., and Moore, I. (2006). Ratiometric Fluorescence-Imaging Assays of Plant Membrane Traffic Using Polyproteins. Traffic 7: 1701-1723.

Sauer, M., Delgadillo, M.O., Zouhar, J., Reynolds, G.D., Pennington, J.G., Jiang, L., 
Liljegren, S.J., Stierhof, Y.-D., De Jaeger, G., Otegui, M.S., Bednarek, S.Y., and Rojo, E. (2013). MTV1 and MTV4 Encode Plant-Specific ENTH and ARF GAP Proteins That Mediate Clathrin-Dependent Trafficking of Vacuolar Cargo from the Trans-Golgi Network. The Plant Cell 25: 2217-2235.

Shevell, D.E., Kunkel, T., and Chua, N.-H. (2000). Cell Wall Alterations in the Arabidopsis emb30 Mutant. Plant Cell 12: 2047-2059.

Shevell, D., Leu, W., Gilimor, C. S., Xia, G., Feldmann, K., and Chua, N. (1994). EMB30 is essential for normal cell division, cell expansion, and cell adhesion in Arabidopsis and encodes a protein that has similarity to Sec7. Cell 77: 1051-1062.

Sieburth, L.E., Muday, G.K., King, E.J., Benton, G., Kim, S., Metcalf, K.E., Meyers, L., Seamen, E., and Norman, J.M. Van (2006). SCARFACE Encodes an ARF-GAP That Is Required for Normal Auxin Efflux and Vein Patterning in Arabidopsis. 18: 1396-1411.

Singh, M.K. and Jürgens, G. (2017). Specificity of plant membrane trafficking - ARFs, regulators and coat proteins. Semin. Cell Dev. Biol.

Stalder, D. and Antonny, B. (2013). Arf GTPase regulation through cascade mechanisms and positive feedback loops. FEBS Letters 587: 2028-2035.

Stefano, G., Renna, L., Rossi, M., Azzarello, E., Pollastri, S., Brandizzi, F., Baluska, F., and Mancuso, S. (2010). AGD5 is a GTPase-activating protein at the trans-Golgi network. The Plant Journal 64: 790-799.

Steinmann, T., Geldner, N., Grebe, M., Mangold, S., Jackson, C. L., Paris, S., Gälweiler, L., Palme, K., and Jürgens, G. (1999). Coordinated Polar Localization of Auxin Efflux Carrier PIN1 by GNOM ARF GEF. Science (80-. ). 286: 316-318.

Steynen, Q.J. and Schultz, E.A. (2003). The FORKED genes are essential for distal vein meeting in Arabidopsis. Development: 4695-4708.

Tanaka, H., Kitakura, S., De Rycke, R., De Groodt, R., and Friml, J. (2009). Fluorescence Imaging-Based Screen Identifies ARF GEF Component of Early Endosomal Trafficking. Current Biology 19: 391-397.

Teh, O. and Moore, I. (2007). An ARF-GEF acting at the Golgi and in selective endocytosis in polarized plant cells. Nature 448: 493-496.

Verna, C., Ravichandran, S.J., Sawchuk, M.G., Linh, M., and Scarpella, E. (2019). Coordination of Tissue Cell Polarity by Auxin Transport and Signaling. Elife.

Wachsman, G., Zhang, J., Moreno-Risueno, M.A., Anderson, C.T., and Benfey, P.N. (2020). Cell wall remodeling and vesicle trafficking mediate the root clock in Arabidopsis. Science 370: 819-823.

Weijers, D., Nemhauser, J., and Yang, Z. (2018). Auxin: small molecule, big impact. Journal of Experimental Botany 69: 133-136. 
Willige, B.C., Ahlers,S., Zourelidou, M., Barbosa, I.C.R., Demarsy, E., Trevisan, M., Davis, P.A., Roelfsema, M.R.G., Hangarter, R., Fankhauser, C., and Schwechheimer, C. (2013). D6PK AGCVIII kinases are required for auxin transport and phototropic hypocotyl bending in Arabidopsis. Plant Cell 25:1674-1688.

Wolters, H., Anders, N., Geldner, N., Gavidia, R., and Jurgens, G. (2011). Coordination of apical and basal embryo development revealed by tissue-specific GNOM functions. Development 138: 117-126.

Wright, J., Kahn, R.A., and Sztul, E. (2014). Regulating the large Sec7 ARF guanine nucleotide exchange factors: the when, where and how of activation. Cell. Mol. Life Sci. 71: 3419-3438.

Xue, S., Zou, J., Liu, Y., Wang, M., and Zhang, C. (2019). Involvement of BIG5 and BIG3 in BRI1 Trafficking Reveals Diverse Functions of BIG-subfamily ARF-GEFs in Plant Growth and Gravitropism. Int. J. Mol. Sci. 20: 2339.

Yorimitsu, T., Sato, K., and Takeuchi, M. (2014). Molecular mechanisms of Sar/Arf GTPases in vesicular trafficking in yeast and plants. Front. Plant Sci. 5: 411.

Zhang, X. et al. (2020). Arabidopsis Flippases Cooperate with ARF GTPase Exchange Factors to Regulate the Trafficking and Polarity of PIN Auxin Transporters. The Plant Cell 32: 1644-1664.

\section{Figure legends}

Figure 1. GN localizes to the PM at often stable punctate structures

(A) CLSM images of GN-GFP and GNL1-GFP in the epidermis of seedling RAMs. In contrast with GNL1-GFP, GN-GFP localizes to the PM. Numbers in bottom right indicate ratios of RAMs with PM signals. Images are representative of data from two independent transgenic lines. Scale bar - $10 \mu \mathrm{m}$.

(B) TIRF images of GN-GFP and GNL1-GFP in the epidermis of early elongation zone of seedling roots. In contrast with GNL1-GFP, the PM signal of GN-GFP consists of relatively bright punctate structures (arrowhead). Both GN-GFP and GNL1-GFP are seen at the GA (large signals) and are present as dynamic, weakly labelled entities likely located in the cytoplasm. Scale bar $-2 \mu \mathrm{m}$.

(C) Kymograph maximum projections of $15 \mu \mathrm{m}$ sections from TIRF movies of GN-GFP and GNL1-GFP, showing GN-GFP-positive punctate structures (arrowhead). The structures are typically characterized by high stability and tend to exhibit limited lateral movement in the plane of the PM. Presence of signals exhibiting any stability is rare in GNL1-GFP. Thick, irregular shapes in both movies are traces of GA moving in the cytoplasm. 
(D) TIRF images and kymograph maximum projections of $15 \mu \mathrm{m}$ sections of TIRF movies of

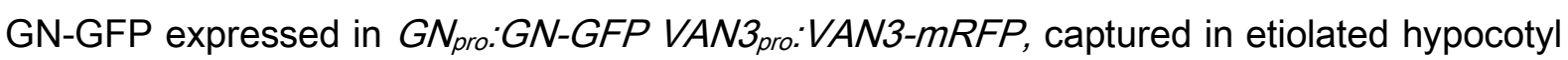
epidermis following a treatment with BFA at $50 \mu \mathrm{M}$ for $1 \mathrm{~h}$. After a treatment with BFA, the stable structures are mostly lost, and punctate signals of high density and typically brief lifetimes are observed. Scale bar - $2 \mu \mathrm{m}$.

\section{Figure 1-figure supplement 1. $g n^{s}$ mutant complementation data}

Seedlings (A) and adults (B) of $g n^{s}$ complemented by $G N_{\text {pro: }}$ GN-GFP. No complementation of $g n^{s}$ by $G N_{\text {pro: }}$ GNL 1-GFP(C).

(D) Root hair positioning in $g n^{s}$ complemented by $G N_{\text {pro: }} G N-G F P, G N_{\text {pro: }} G N^{\text {wwr }}$ GFP and

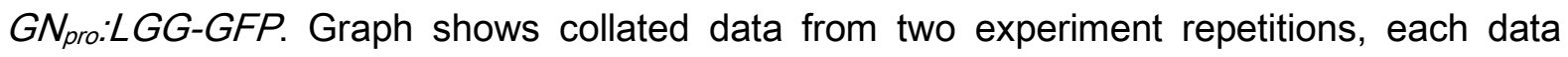
point representing one root hair. Numbers indicate means \pm s.d. Scale bar $-20 \mu \mathrm{m}$.

(E) Root lengths of $8 \mathrm{~d}$ old $g n^{s}$ complemented by $G N_{\text {pro: }}$ GN-GFP and $G N_{\text {pro: }}$ GNiwr_GFP. Col0: $3.82 \pm 0.99 \mathrm{~cm}, \mathrm{n}=32 ; G N_{\text {pro: }}$ GN-GFP L1: $3.85 \pm 0.74 \mathrm{~cm}, \mathrm{n}=34, \mathrm{~L} 2: 4.52 \pm 0.36 \mathrm{~cm}$, n=32; GN pro:GNiwr_GFP L1: $4.03 \pm 1.07 \mathrm{~cm}, \mathrm{n}=29$, L2: $4.37 \pm 1.07 \mathrm{~cm}, \mathrm{n}=29$. Values were compared using One-way ANOVA $(\mathrm{P}=0.0038)$ with post-hoc Tukey HSD test, groups of significantly different values are indicated.

Figure 1-figure supplement 2. GN-GFP and GNL1-GFP subcellular localization data from two transgenic lines analyzed

CLSM (A) and TIRF (B) images of GN-GFP and GNL1-GFP from two transgenic lines analyzed. Scale bars - (A) $10 \mu \mathrm{m}$, (B) $2 \mu \mathrm{m}$.

Figure 1-figure supplement 3. TIRF colocalization of GN-GFP and VAN3-mRFP following a BFA treatment

TIRF colocalization of GN-GFP with VAN3-mRFP in etiolated hypocotyl epidermis, following a treatment with BFA at $50 \mu \mathrm{M}$ for $1 \mathrm{~h}$. The BFA-induced, dense and dynamic punctate signals of GN-GFP do not evidently colocalize with VAN3-mRFP at the PM. Scale bar - 2 $\mu \mathrm{m}$.

Figure 1-figure supplement 4. Quantitative data on GN-positive PM structures

(A) Lateral displacement of individual GN-GFP and GNfwr_GFP structures estimated from kymograph maximum projections of TIRF movies. GN-GFP: $0.555 \pm 0.280 \mu \mathrm{m}$ (mean \pm s.d.), GN'fwr-GFP: $0.566 \pm 0.264 \mu \mathrm{m}$. ns - not significant. 
(B) Density of GN-GFP signals in control conditions and following a treatment with BFA at 50 $\mu \mathrm{M}$ for 1 h. Control: $8.29 \pm 3.20$ foci per $100 \mu \mathrm{m}^{2}$ (mean \pm s.d.), BFA: $37.49 \pm 9.14$ foci per $100 \mu \mathrm{m}^{2}$. ${ }^{* *} \mathrm{P}<0.001$.

(C) Density of GN-GFP and GNwwr-GFP signals. GN-GFP: $9.11 \pm 3.51$ foci per $100 \mu \mathrm{m}^{2}$ (mean \pm s.d.), GNfwr-GFP: $12.40 \pm 4.19$ foci per $100 \mu \mathrm{m}^{2}$. ${ }^{* *} \mathrm{P}<0.01$.

Figure 2. A functional GNiwr-GFP variant localizes to punctate structures at the PM, but not to the GA

(A) $8 \mathrm{~d}$ old seedlings of $g n^{s}$ mutants complemented with $G N_{\text {pro: }}: G N-G F P$ and $G N_{\text {pro: }} G N^{\text {wwr }}$ GFP transgenes. Two independent lines of each complemented mutant are shown.

(B) Adult $g n^{s}$ mutants complemented with $G N_{\text {pro: }}$ GN-GFP and $G N_{\text {pro: }}$ GNwr_GFP transgenes.

(C) and (D) TIRF images of GN-GFP and GNfwr_GFP expressed in complemented $g n^{s}$ background in hypocotyls of etiolated seedlings $(C)$ and in early elongation zone of seedling roots (D). GNiwr localizes to GN-specific structures at the PM, but not to the GA. Scale bars $2 \mu \mathrm{m}$.

(E) Kymograph maximum projection of a $15 \mu \mathrm{m}$ section from a TIRF movie of GN fwr-GFP showing stable, PM-localized punctate structures.

Figure 2-figure supplement 1. Subcellular localization of GN-GFP and GNiwr-GFP at basal end of young trichoblasts

TIRF images (A) and kymographs (B) of GN-GFP and GN fwr-GFP expressed in complemented mutant backgrounds, at basal ends of young trichoblasts. Scale bar $-2 \mu \mathrm{m}$.

Figure 3. GN-positive PM structures do not contain clathrin, and CME is normal in $g^{s}$ TIRF images (A) and kymographs (B) of GN-GFP and CLC-mKO in the epidermis of early elongation zone of seedling roots. The GN-positive structures at the PM are distinct from CCPs. Scale bar - $2 \mu \mathrm{m}$. TIRF images of CLC2-GFP (C) and TPLATE-GFP (E) in middle regions of etiolated $g n^{s}$ seedlings and in hypocotyls of etiolated wild type controls. Scale bars - $2 \mu \mathrm{m}$. Graphs show quantifications of foci densities at the PM, each data point representing a measurement from a single movie. CLC2-GFP wild-type: $123 \pm 17$ foci per $100 \mu \mathrm{m}^{2}$ (mean \pm s.d.), $\mathrm{n}=10 ; g n^{s:} 121 \pm 20$ foci per $100 \mu \mathrm{m}^{2}, \mathrm{n}=16$. TPLATE-GFP wild-type: $121 \pm 18$ foci per $100 \mu m^{2}, n=14 ; g n^{s}: 115 \pm 25$ foci per $100 \mu m^{2}, n=16$. Values were compared using $t$ tests, ns - not significant. Kymographs from TIRF movies representing dynamics of CLC2-GFP (D) and TPLATE-GFP (F) in middle regions of etiolated $g n^{s}$ seedlings and in hypocotyls of etiolated wild type controls. Histograms show distributions of 
lifetimes of single endocytic events. CLC2-GFP wild type $n=146, g n^{s} n=150$; TPLATE-GFP wild type $n=205, g n^{s} \mathrm{n}=137$.

Figure 3-figure supplement 1. Control TIRF co-localization of CME markers TPLATE-GFP and AP2A1-TagRFP

TIRF images (A) and kymographs (B) of TPLATE-GFP and AP2A1-TagRFP in the epidermis of early elongation zone of seedling roots. Scale bar $-2 \mu \mathrm{m}$. Arrowheads in $(A)$ indicate colocalizing punctae.

\section{Figure 4. Bulk secretion is normal in $g n^{s}$}

(A) Maximum projections of z-stacks of CLSM images of secRFP in RAMs of wild type seedlings and in basal ends of $g n^{s}$ seedlings. Scale bars - $20 \mu \mathrm{m}$. (B) Maximum projection of a z-stack of CLSM images of secRFP in a whole $g n^{s}$ seedling. Scale bar $-200 \mu \mathrm{m}$.

Figure 4-figure supplement 1. Additional controls for secRFP secretion

(A) CLSM maximum projection of a z-stack of secRFP in RAMs of $2 \mathrm{~d}$ old wild type seedlings. (B) Maximum projections of z-stacks of CLSM images in $g n^{s}$ basal ends with and without secRFP expression. Scale bars - $10 \mu \mathrm{m}$.

Figure 5. A BFA-induced, GN-specific exocytic event

(A) CLSM images of GN-GFP in seedling RAM epidermis following a treatment with BFA at $50 \mu \mathrm{M}$ for $1 \mathrm{~h}$. After a treatment with BFA, GN-GFP locates to the core of BFA bodies and strongly localizes to the PM. Scale bar - $10 \mu \mathrm{m}$.

(B) CLSM images of GN-GFP in seedling RAM epidermis of big3 and of induced $X V E$ »amiCHCa, following treatments with BFA at $50 \mu \mathrm{M}$ for $1 \mathrm{~h}$. The relocation of GN-GFP to the PM is abolished to a large degree in big3 and completely in XVE»amiCHCa. Scale bars - $10 \mu \mathrm{m}$. Treatments of non-induced XVE»amiCHCa lines are shown in Figure 5-figure supplement 1B.

(C) CLSM images of GNwrr-GFP in seedling RAM epidermis following a treatment with BFA at $50 \mu \mathrm{M}$ for $1 \mathrm{~h}$. After a treatment with BFA, GNiwr-GFP binds to the core of BFA bodies but does not localize to the PM. Scale bar - $10 \mu \mathrm{m}$.

(D) CLSM images of a BFA-sensitive variant of GNL1-YFP in seedling RAM epidermis following a treatment with BFA at $50 \mu \mathrm{M}$ for $1 \mathrm{~h}$. The images show GNL1sens-YFP expressed in a wild type background in gn/1 GNL1 pro:GNL 1sens-YFP $\times$ Col-0 F1. After a treatment with BFA, GNL1 1sens-YFP locates to the core of BFA bodies and weakly to the PM. Scale bar - 10 $\mu \mathrm{m}$. 
(E) Graph showing quantifications of PM signal intensities relative to total RAM signals of GN-GFP in wild type and big3 mutants, of GNL1sens_YFP, and of GNwrr-GFP, following treatments with BFA at $50 \mu \mathrm{M}$ for $1 \mathrm{~h}$. GN-GFP wild type: $1.39 \pm 0.11$ (mean \pm s.d.), $\mathrm{n}=37$;

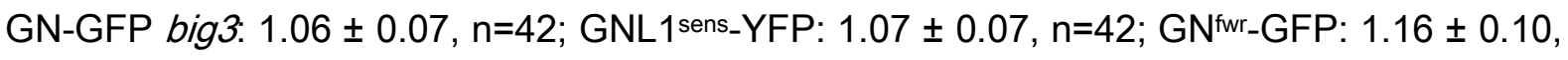
$n=40$. The graph has an indicative purpose only, as comparison of quantified ratios does not reflect the visual observations precisely. This is due to differences in the samples such as the expression of different marker proteins, or alterations in BFA body structures in big3.

(F) Graph showing quantifications of PM signal intensities relative to total RAM signals of GN-GFP following treatments with BFA at $50 \mu \mathrm{M}$ for $1 \mathrm{~h}$ in control and induced XVE»amiCHCa lines. Mock: $1.56 \pm 0.07$ (mean \pm s.d.), $n=30$; induced: $1.18 \pm 0.12, n=27$. Values were compared using a $t$ test, $\mathrm{P}<0.0001$.

\section{Figure 5-figure supplement 1. A BFA-induced, GN-specific exocytic event}

(A) A CLSM image of GN-GFP in seedling RAM epidermis following a treatment with BFA at $50 \mu \mathrm{M}$ for $1 \mathrm{~h}$, demonstrating strong localization of GN-GFP to the cell plates. Scale bar -10 $\mu \mathrm{m}$.

(B) Control CLSM images of GN-GFP in seedling RAM epidermis of non-induced XVE»amiCHCa line following treatments with BFA at $50 \mu \mathrm{M}$ for $1 \mathrm{~h}$. Scale bar $-10 \mu \mathrm{m}$.

(C) CLSM images of native GNL1-GFP in seedling RAM epidermis following a treatment with BFA at $50 \mu \mathrm{M}$ for $1 \mathrm{~h}$. After a treatment with BFA, native GNL1-GFP remains at the GA at the periphery of BFA bodies. Scale bar $-10 \mu \mathrm{m}$.

(D) CLSM images of a BFA-sensitive variant of GNL1-YFP in seedling RAM epidermis following a treatment with BFA at $50 \mu \mathrm{M}$ for $1 \mathrm{~h}$. The images show GNL1 ${ }^{\text {sens-YFP expressed }}$ in $g n / 1$ background. After a treatment with BFA, typical BFA bodies are not formed, and GNL1sens-YFP relocates to the PM. Scale bar - $10 \mu \mathrm{m}$.

(E) CLSM images of VHAa1-GFP in seedling RAM epidermis of wild type and gn/1-2 mutants following a treatment with BFA at $25 \mu \mathrm{M}$ for $1 \mathrm{~h}$. After a treatment with BFA, VHAa1GFP is found at the core of BFA bodies in the wild type, but undergoes relocation to the PM in $g n / 1-2$. Scale bar - $10 \mu \mathrm{m}$.

\section{Figure 6. GNOM - GNOM-LIKE1 chimeric ARF-GEFs}

(A) Schematic representation of the domain composition of GN, GNL1, and chimeric GBF1type ARF-GEFs. An LLG chimera was not cloned.

(B) No complementation of $g n^{s}$ by the expression of GLL-GFP.

(C) Phenotypes of adult $g n^{s}$ mutants complemented by the expression of GN-GFP, GGLGFP, LGG-GFP, and GLG-GFP. Expression of GGL-GFP and LGG-GFP fully complements 
the mutant phenotype, while plants expressing GLG-GFP develop smaller rosettes, fewer branches, and have limited fertility.

(D) Phenotypes of adult $g n^{s}$ mutants complemented by the expression of LGL-GFP. Expression of LGL-GFP provides only a modest level of complementation. Adults are characterized by limited growth, a compact rosette, and if bolting, are completely infertile. Wild type siblings from a population segregating for $g n^{s}$ are shown as control.

Photographs in (C) and (D) show two independent transgenic lines expressing each construct.

Figure 6-figure supplement 1. Additional data related to GNOM - GNOM-LIKE1 chimeras

(A) Terminal phenotypes of $g n^{s}$ mutants complemented by the expression of LGL-GFP.

(B) An additional picture of adult $g n^{s}$ mutants complemented by the expression of GLG-GFP.

(C) Control images showing etiolated seedlings of wild type plants and $g n^{s}$ mutants complemented by the expression of GN-GFP.

Figure 7. Seedling phenotypes of $g n^{s}$ mutants complemented with GGL-GFP, LGG-GFP, and GLG-GFP.

(A) Seedlings of $g n^{s}$ mutants expressing LGG-GFP and GGL-GFP. Expression of LGG-GFP fully complements the mutant phenotype, while seedlings expressing GGL-GFP are characterized by roots growing more straight than controls.

(B) Seedlings of $g n^{s}$ mutants expressing GGL-GFP and LGG-GFP growing on agar plates tilted back from the vertical position. The root waving phenotype is normal in seedlings expressing LGG-GFP, while seedlings expressing GGL-GFP exhibit decreased waving.

(C) Seedlings of $g n^{s}$ mutants expressing GLG-GFP and LGL-GFP. Seedlings expressing GLG-GFP have moderately and variably decreased root lengths, while seedlings expressing LGL-GFP have strongly decreased root lengths. Both lines exhibit single or partially fused cotyledons in up to $50 \%$ of seedlings (GLG-GFP: right panel; LGL-GFP: bottom panel).

(D) Seedlings of $g n^{s}$ mutants expressing GLG-GFP and LGL-GFP growing on agar plates tilted back from the vertical position. The root waving phenotype is less expressed in mutants complemented with GLG-GFP, and completely absent in mutants complemented with LGL-GFP. The rightmost panel shows a population segregating for $g n^{s}$, complemented homozygous mutants are marked by asterisks.

(E) Detail of single cotyledon phenotypes of $g n^{s}$ mutants complemented by the expression of GLG-GFP and LGL-GFP. 
(F) Etiolated seedlings of $g n^{s}$ mutants expressing GLG-GFP and LGL-GFP exhibit agravitropic growth. The bottom panel shows a population segregating for $g n^{s}$, complemented homozygous mutants are marked by asterisks.

(G) Etiolated seedlings of $g n^{s}$ mutants expressing GLG-GFP and LGL-GFP exhibit open apical hooks at $75 \mathrm{~h}$ of in vitro growth.

\section{Figure 7-figure supplement 1 . The effect of NPA on root waving}

Col-0 seedlings grown on media supplemented with $1 \mu \mathrm{M}$ NPA on vertical and tilted plates. NPA causes loss of root waving as exhibited on plates tilted back from the vertical.

Figure 7-figure supplement 2. Additional data on seedling phenotypes of $g n^{s}$ mutants complemented with GN-GNL1 chimeras

Growth on tilted plates showing both general seedling morphology and root waving $(A)$, single and fused cotyledons (B), dark-grown seedlings (C), and apical hooks (D) of indicated lines.

Figure 8. Subcellular localization and BFA-induced exocytosis of ARF-GEF chimeras (A) TIRF images of GN-GFP, LGG-GFP, GGL-GFP, GLG-GFP and LGL-GFP in hypocotyls of etiolated seedlings of complemented $g n^{s}$ mutants. All complementing chimeras localize to the GN-specific structures at the PM, and to GA. Scale bar - $2 \mu \mathrm{m}$.

(B) TIRF image of GLL-GFP in wild type etiolated seedling hypocotyl. GLL-GFP can be identified at GN-specific structures at the PM, and the GA. Scale bar - $2 \mu \mathrm{m}$.

(C) CLSM images of seedling RAM epidermis of GN-GFP and chimeras possessing the BFA-sensitive SEC7 domain of GN, expressed in wild type background, following a treatment with BFA at $25 \mu \mathrm{M}$ for $1 \mathrm{~h}$. LGG-GFP and GGL-GFP localize to the PM after a BFA treatment, while LGL-GFP does not. Scale bar - $10 \mu \mathrm{m}$. Graph shows a quantification of PM signal intensities relative to total RAM signals following treatments with BFA at $25 \mu \mathrm{M}$ for $1 \mathrm{~h}$ from a representative experiment. A sum of data from two transgenic lines expressing each fluorescent reporter is shown. GN-GFP: $1.79 \pm 0.13$ (mean \pm s.d.), $n=32$; LGG-GFP: $1.46 \pm 0.15, n=29 ;$ GGL-GFP: $1.46 \pm 0.09, n=27$; LGL-GFP: $1.07 \pm 0.12, n=26$. Values were compared using One-way ANOVA $(P<0.0001)$ with post-hoc Tukey HSD test, groups of significantly different values are indicated.

Figure 8-figure supplement 1. Additional data on subcellular localization and BFA-induced exocytosis of ARF-GEF chimeras 
(A) TIRF images of LGG-GFP, GGL-GFP, GLG-GFP and LGL-GFP in hypocotyls of etiolated seedlings of complemented $g n^{s}$ mutants from two transgenic lines tested. Scale bar $-2 \mu \mathrm{m}$.

(B) CLSM images of seedling RAM epidermis of GFP fusions of chimeras possessing the BFA-resistant SEC7 domain of GNL1, expressed in wild type background, following a treatment with BFA at $25 \mu \mathrm{M}$ for $1 \mathrm{~h}$. GLL-GFP and GLG-GFP are retained at the periphery of the BFA body. Scale bar - $10 \mu \mathrm{m}$.

(C) CLSM images in seedling RAM epidermis of two transgenic lines of LGG-GFP, GGLGFP, and LGL-GFP upon a treatment with BFA at $25 \mu \mathrm{M}$ for $1 \mathrm{~h}$. Scale bar $-10 \mu \mathrm{m}$.

\section{Rich media}

Video 1. TIRF time lapse of GN-GFP in seedling root epidermis

Video 2. TIRF time lapse of GNL1-GFP in seedling root epidermis

Video 3. TIRF time lapse of GN-GFP in hypocotyl epidermis following a mock treatment

Video 4. TIRF time lapse of GN-GFP in hypocotyl epidermis following a treatment with BFA $50 \mu \mathrm{M}$ for $1 \mathrm{~h}$

Video 5. TIRF time lapse colocalization of GNOM-GFP and VAN3-mRFP in hypocotyl epidermis following a treatment with BFA $50 \mu \mathrm{M}$ for $1 \mathrm{~h}$, green channel

Video 6. TIRF time lapse colocalization of GNOM-GFP and VAN3-mRFP in hypocotyl epidermis following a treatment with BFA $50 \mu \mathrm{M}$ for $1 \mathrm{~h}$, red channel

Video 7. TIRF time lapse colocalization of GNOM-GFP and VAN3-mRFP in hypocotyl epidermis following a treatment with BFA $50 \mu \mathrm{M}$ for $1 \mathrm{~h}$, merged channels

Video 8. TIRF time lapse of GNfwr_GFP in $g n^{s}$ seedling root epidermis

Video 9. TIRF time lapse colocalization of GN-GFP and CLC-mKO in seedling root epidermis, green channel

Video 10. TIRF time lapse colocalization of GN-GFP and CLC-mKO in seedling root epidermis, red channel

Video 11. TIRF time lapse colocalization of GN-GFP and CLC-mKO in seedling root epidermis, merged channels

Video 12. TIRF time lapse of CLC2-GFP in wild-type hypocotyl epidermis

Video 13. TIRF time lapse of CLC2-GFP in $g n^{s}$ hypocotyl epidermis

Video 14. TIRF time lapse of TPLATE-GFP in wild-type hypocotyl epidermis

Video 15. TIRF time lapse of TPLATE-GFP in $g n^{s}$ hypocotyl epidermis 


\section{Tables}

Table 1. Summary of phenotypes of $g n^{s}$ expressing ARF-GEF variants

\begin{tabular}{|c|c|c|c|c|c|c|c|c|}
\hline ARF-GEF & $\begin{array}{l}\text { overall degree } \\
\text { of } \mathrm{GN} \text { function }\end{array}$ & adult phenotype & seedling growth rate & root waving & $\begin{array}{l}\text { root hair } \\
\text { positioning }\end{array}$ & apical patterning & $\begin{array}{l}\text { etiolated seedling } \\
\text { tropism }\end{array}$ & apical hooks \\
\hline GN (GGG) & complete & normal & normal & normal & normal & normal & normal & normal \\
\hline LGG & complete & normal & normal & normal & normal & normal & normal & normal \\
\hline GLG & moderate & $\begin{array}{l}\text { decreased } \\
\text { growth and } \\
\text { fertility }\end{array}$ & partially decreased & decreased & $\begin{array}{l}\text { not } \\
\text { analyzed }\end{array}$ & $\begin{array}{l}\sim 50 \% \text { single or } \\
\text { fused cotyledons }\end{array}$ & $\begin{array}{l}\text { variable growth } \\
\text { directions }\end{array}$ & $\begin{array}{l}\text { open apical } \\
\text { hooks }\end{array}$ \\
\hline LGL & $\begin{array}{l}\text { moderate to } \\
\text { low }\end{array}$ & $\begin{array}{l}\text { very small, } \\
\text { infertile }\end{array}$ & strongly decreased & absent & $\begin{array}{l}\text { not } \\
\text { analyzed }\end{array}$ & $\begin{array}{l}\sim 50 \% \text { single or } \\
\text { fused cotyledons }\end{array}$ & $\begin{array}{l}\text { variable growth } \\
\text { directions }\end{array}$ & $\begin{array}{l}\text { open apical } \\
\text { hooks }\end{array}$ \\
\hline GLL & none & - & identical to $g n$ & - & - & identical to $g n$ & - & - \\
\hline GNL1 (LLL) & none & - & identical to $g n$ & - & - & identical to $g n$ & - & - \\
\hline LLG & & & & & & & & \\
\hline
\end{tabular}

\section{Supplementary File legends}

Supplementary File 1. Additional materials and methods.

Supplementary File 1a. Lines generated as part of this study.

Supplementary File 1b. Primers used in this study.

Supplementary File 1c. Constructs generated in this study. 
Figure 1

A

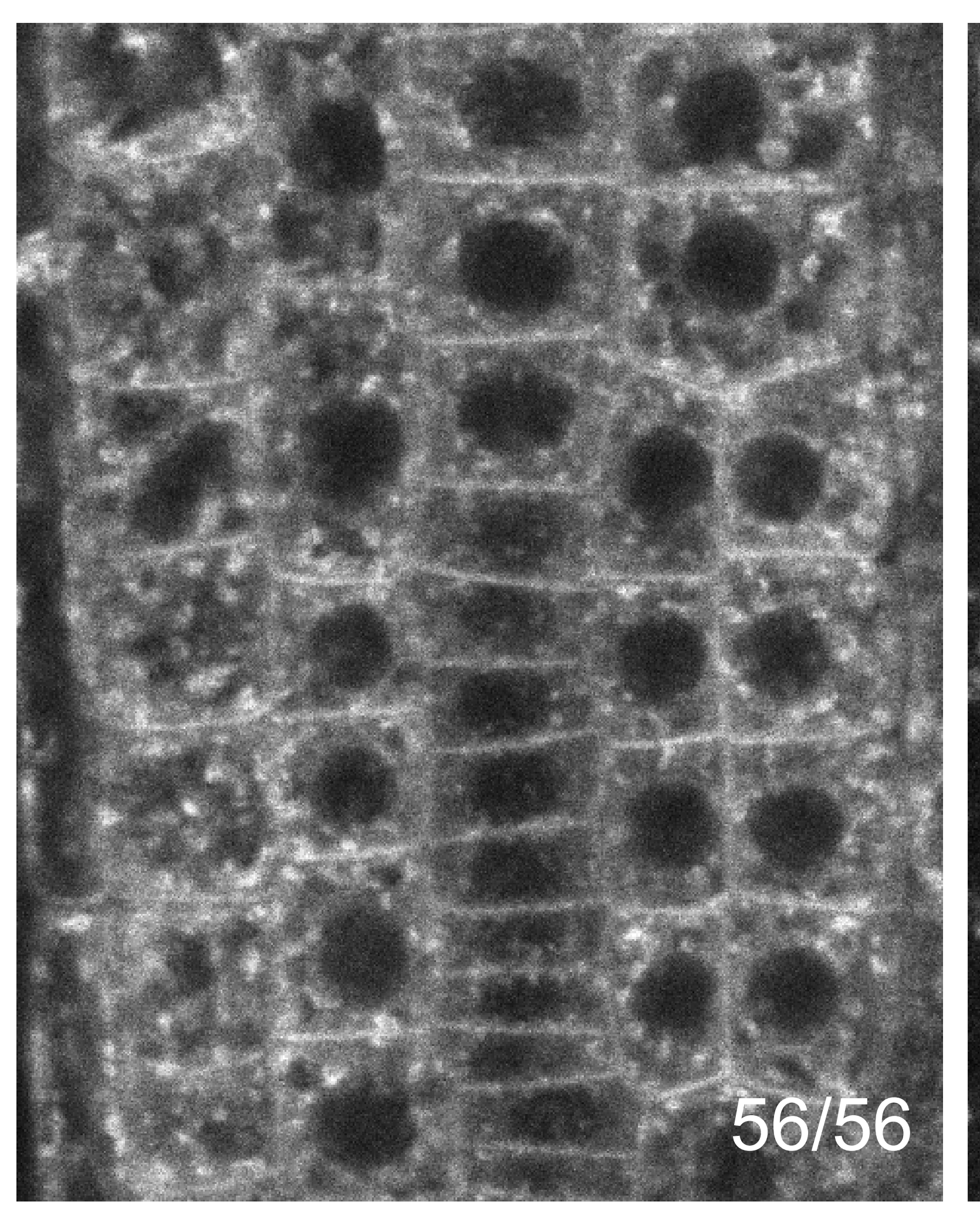

$G N_{\text {pro }}: G N-G F P$

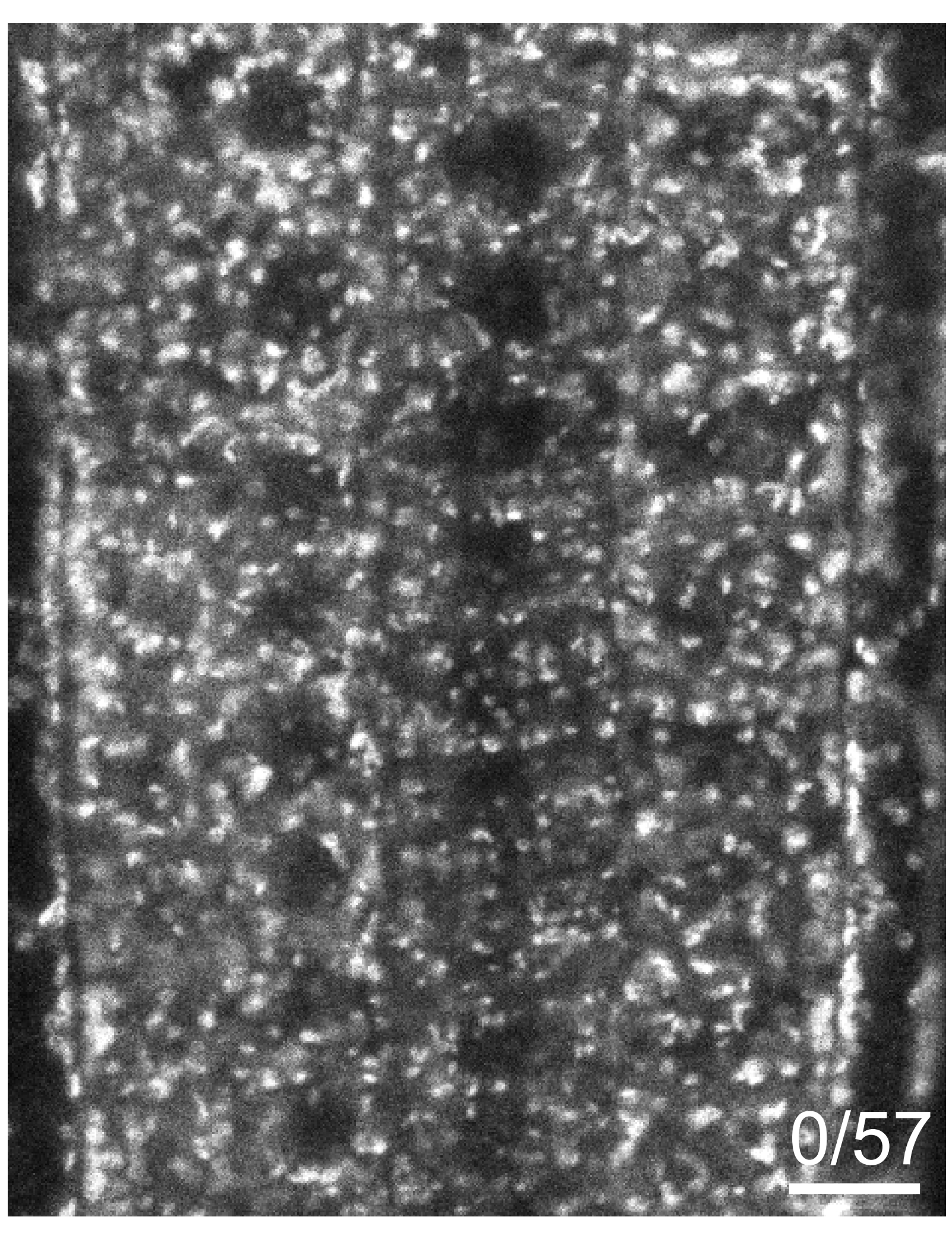

$G N_{\text {pro }}: G N L 1-G F P$
B

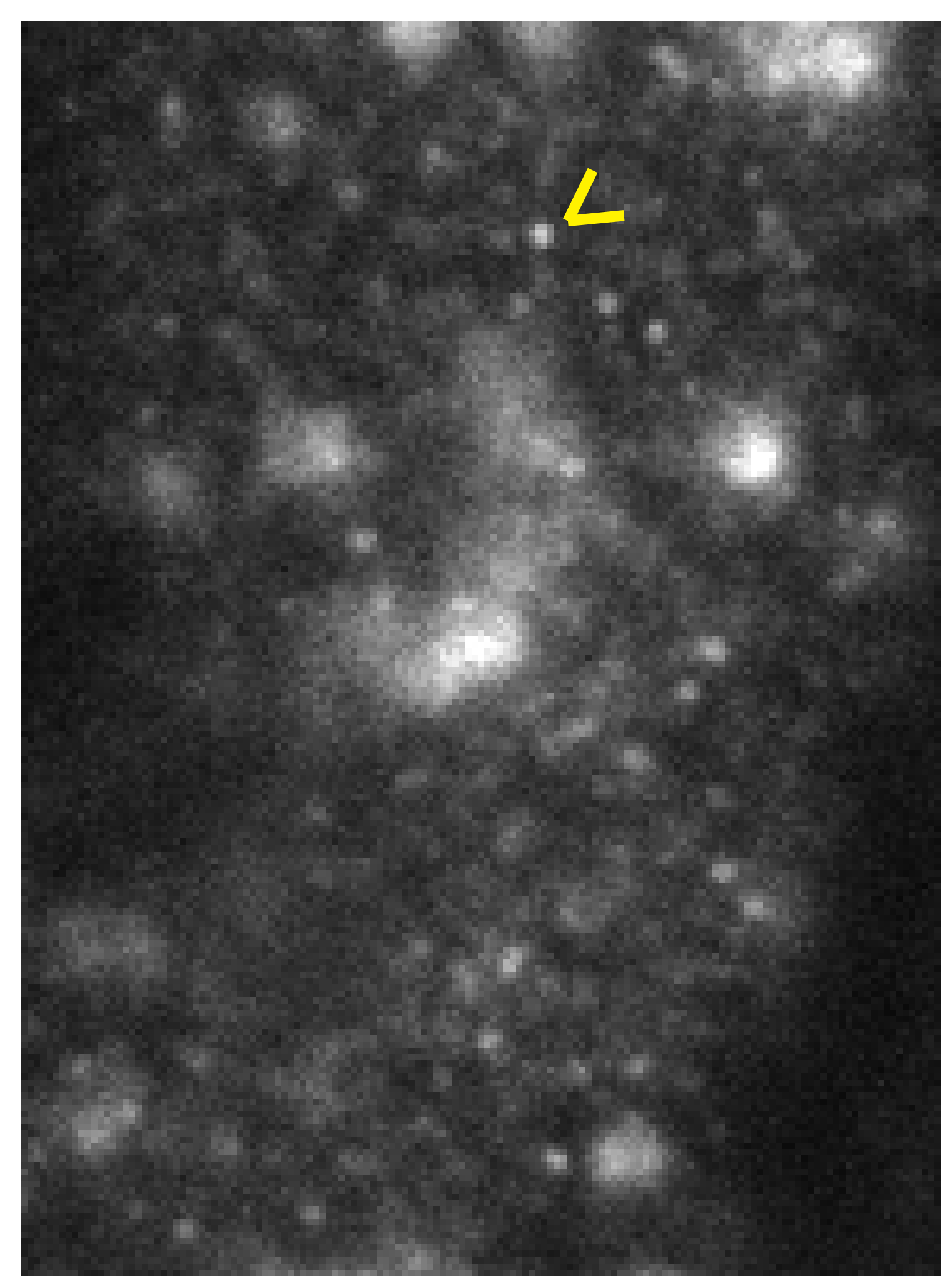

$G N_{\text {pro: }}: G N-G F P$

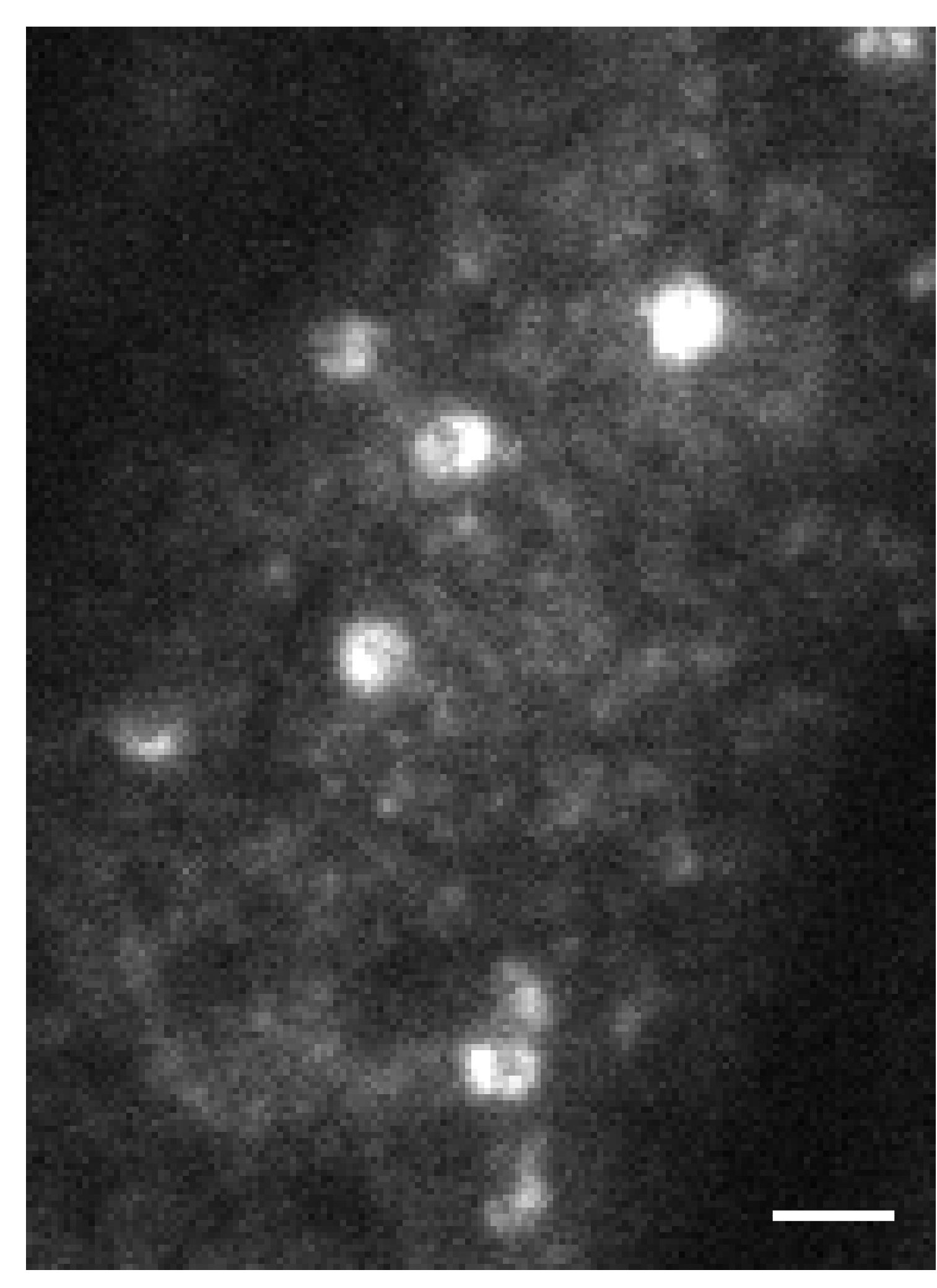

$G N_{\text {pro: }}: G N L 1-G F P$

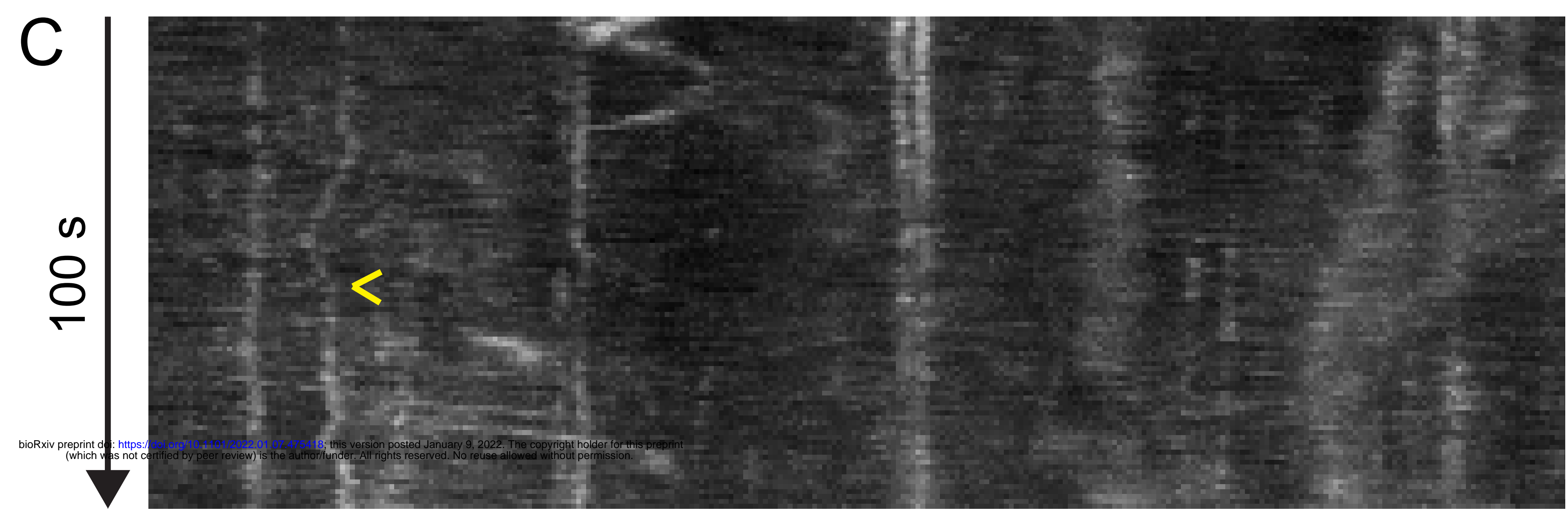

$G N_{\text {pro }}: G N-G F P$

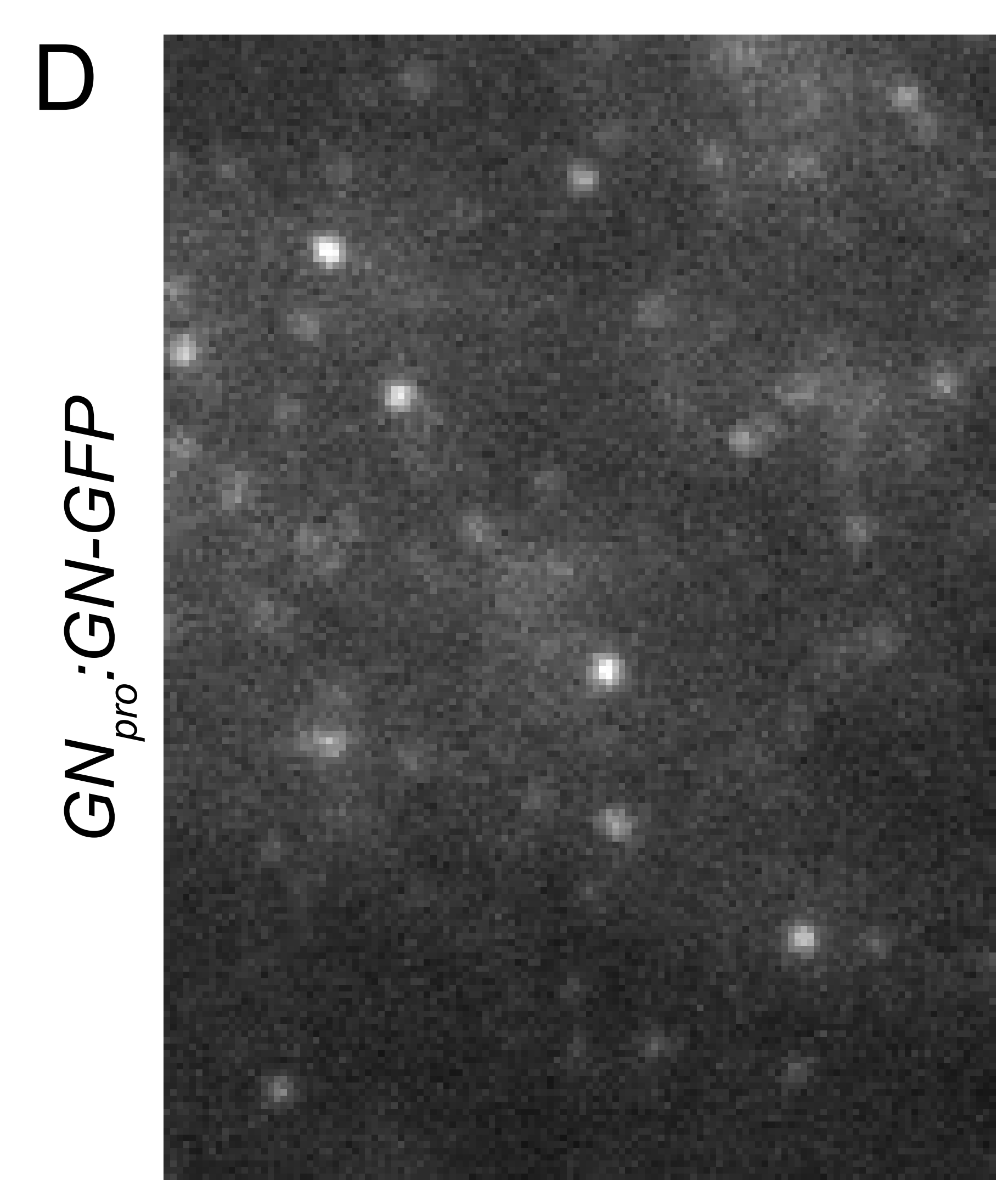

mock

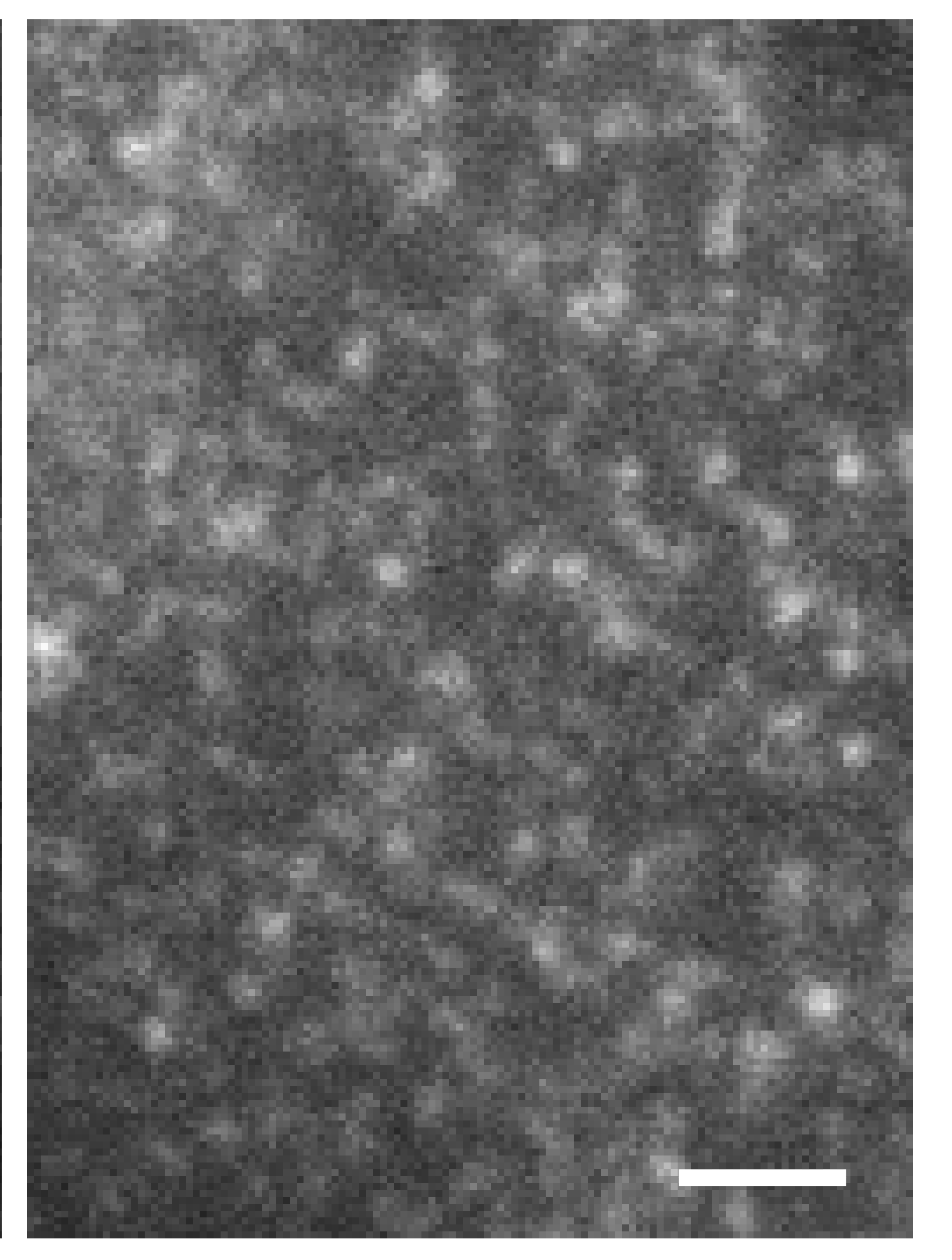

BFA $50 \mu \mathrm{M} 1 \mathrm{~h}$

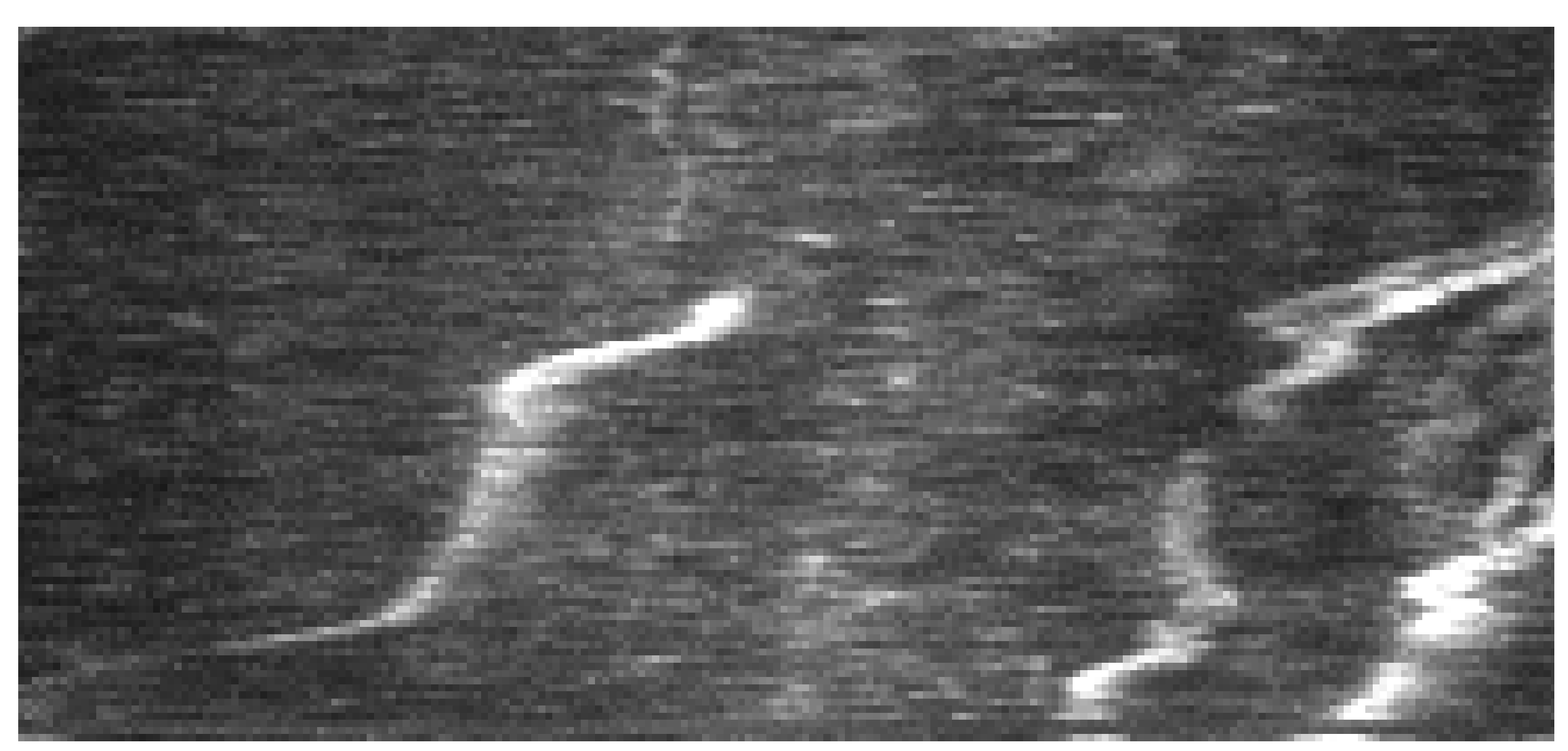

$G N_{p r o}: G N L 1-G F P$

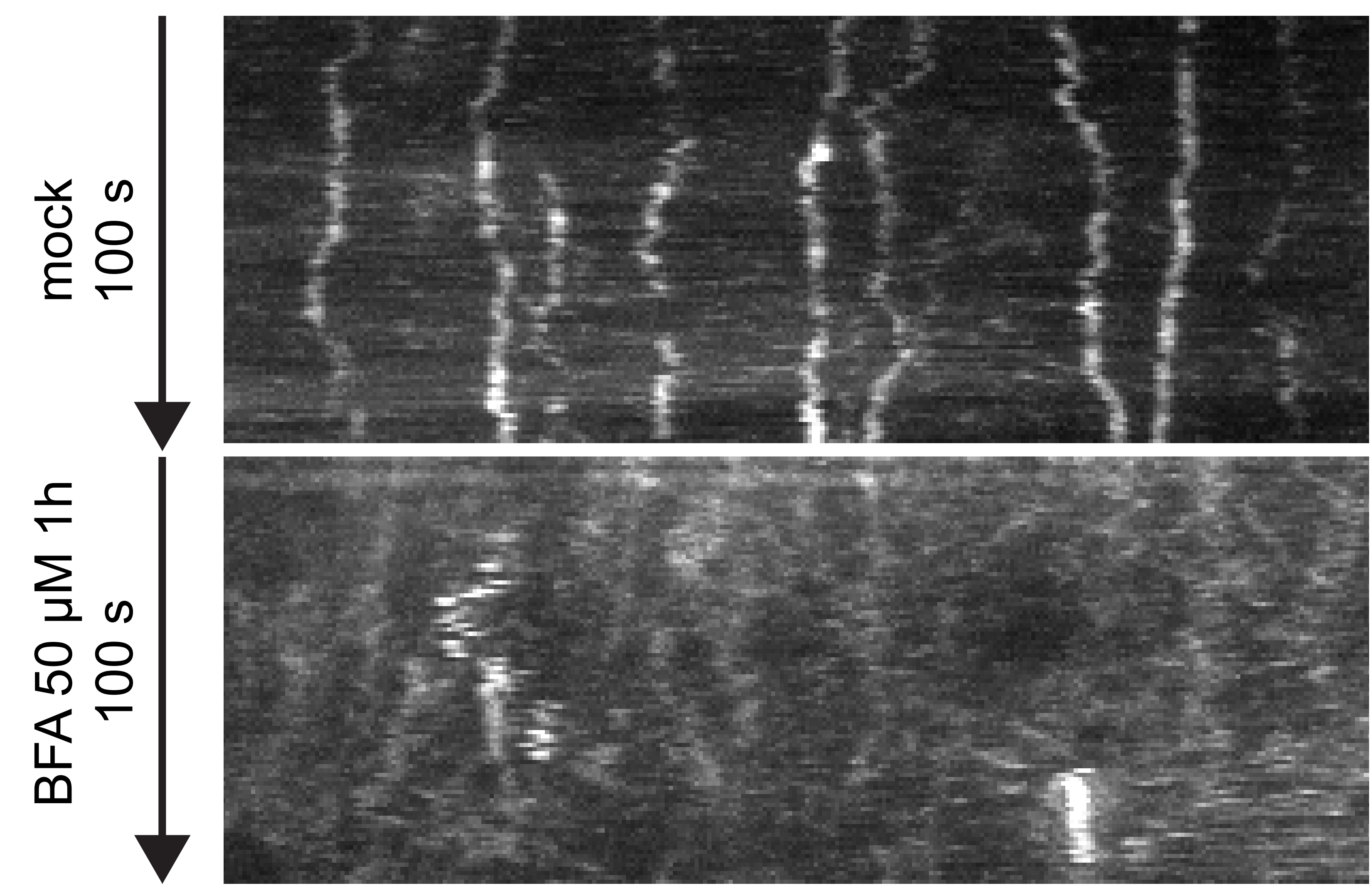


Figure 1-figure supplement 1

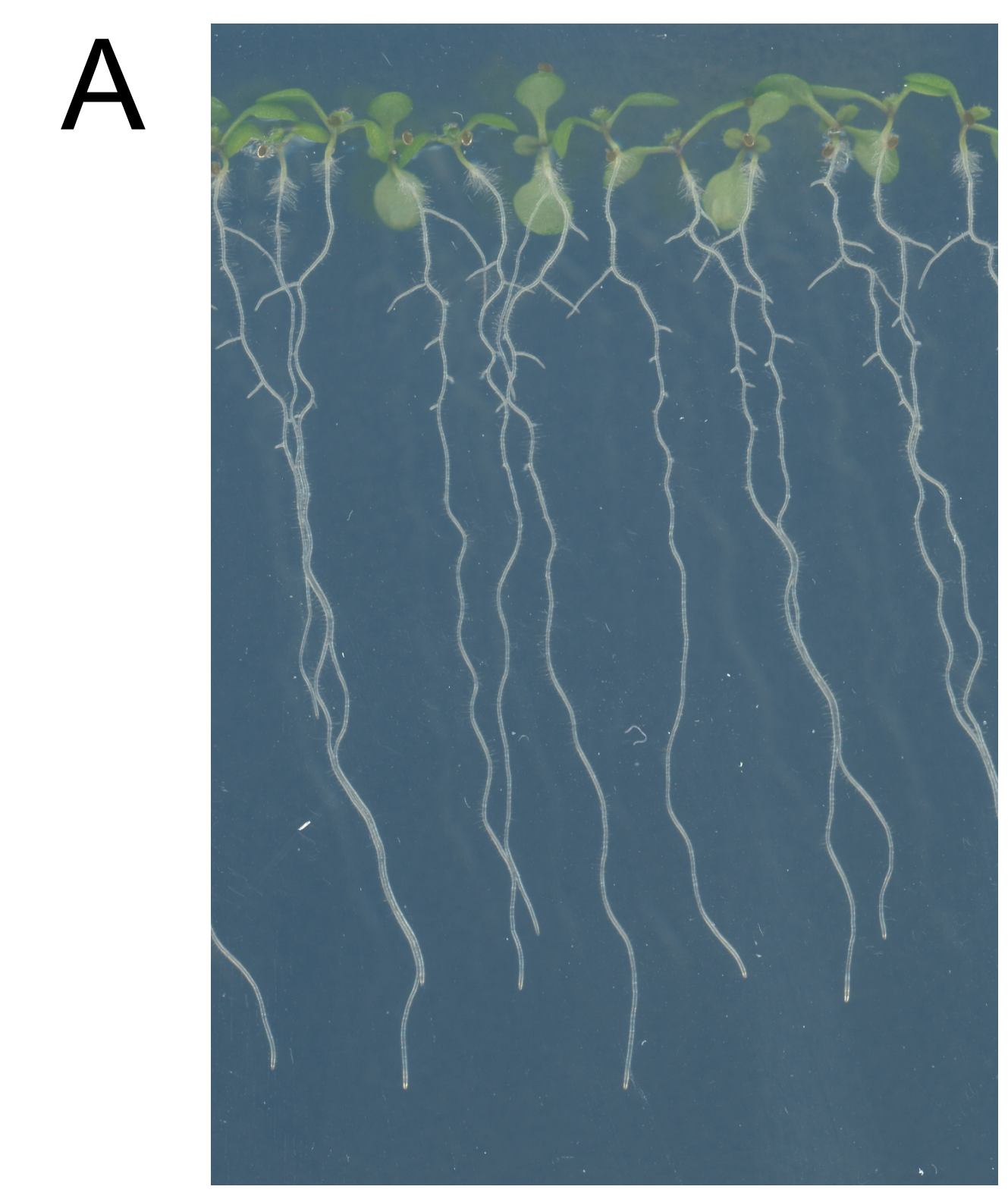

Col-0
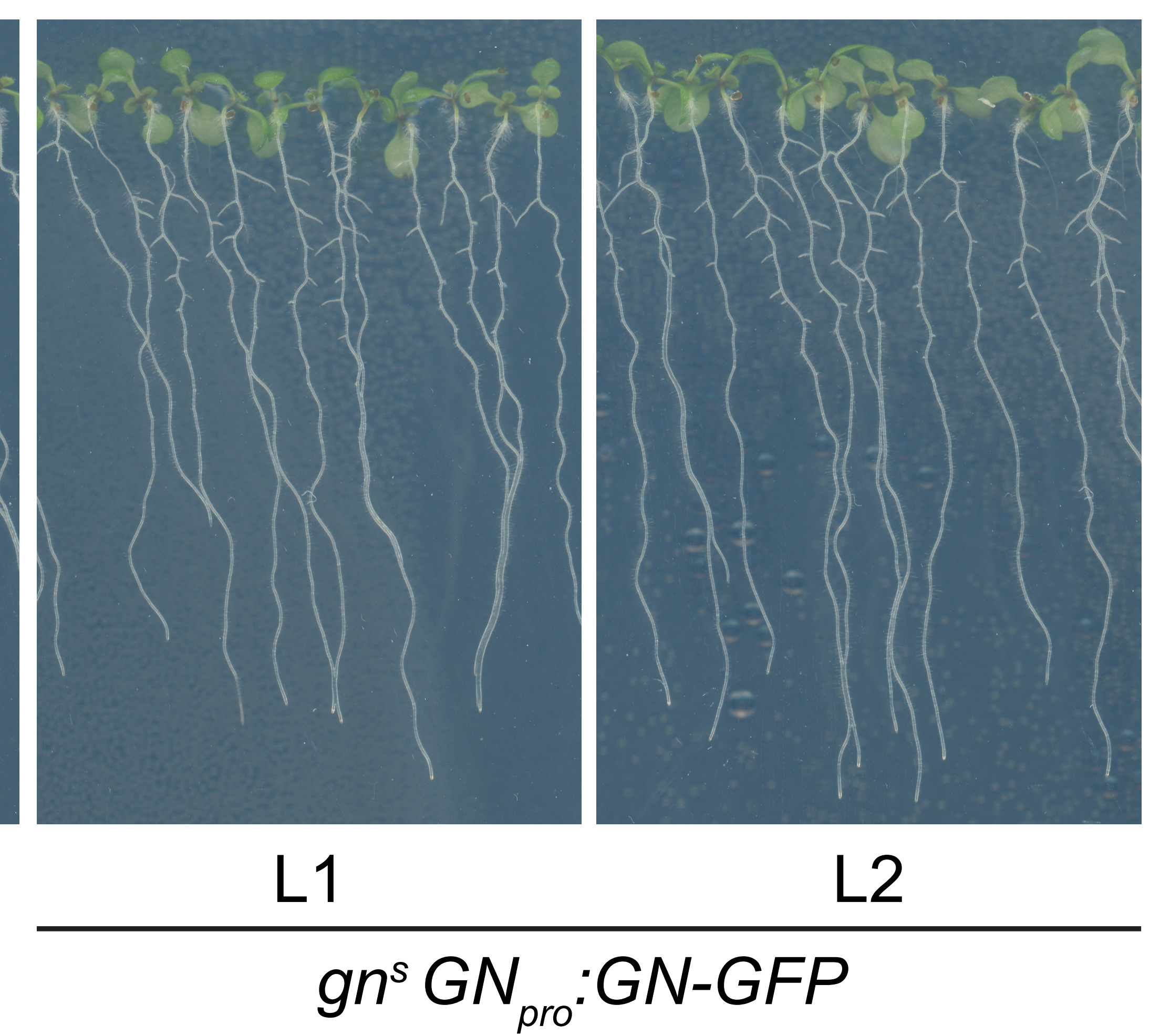

C

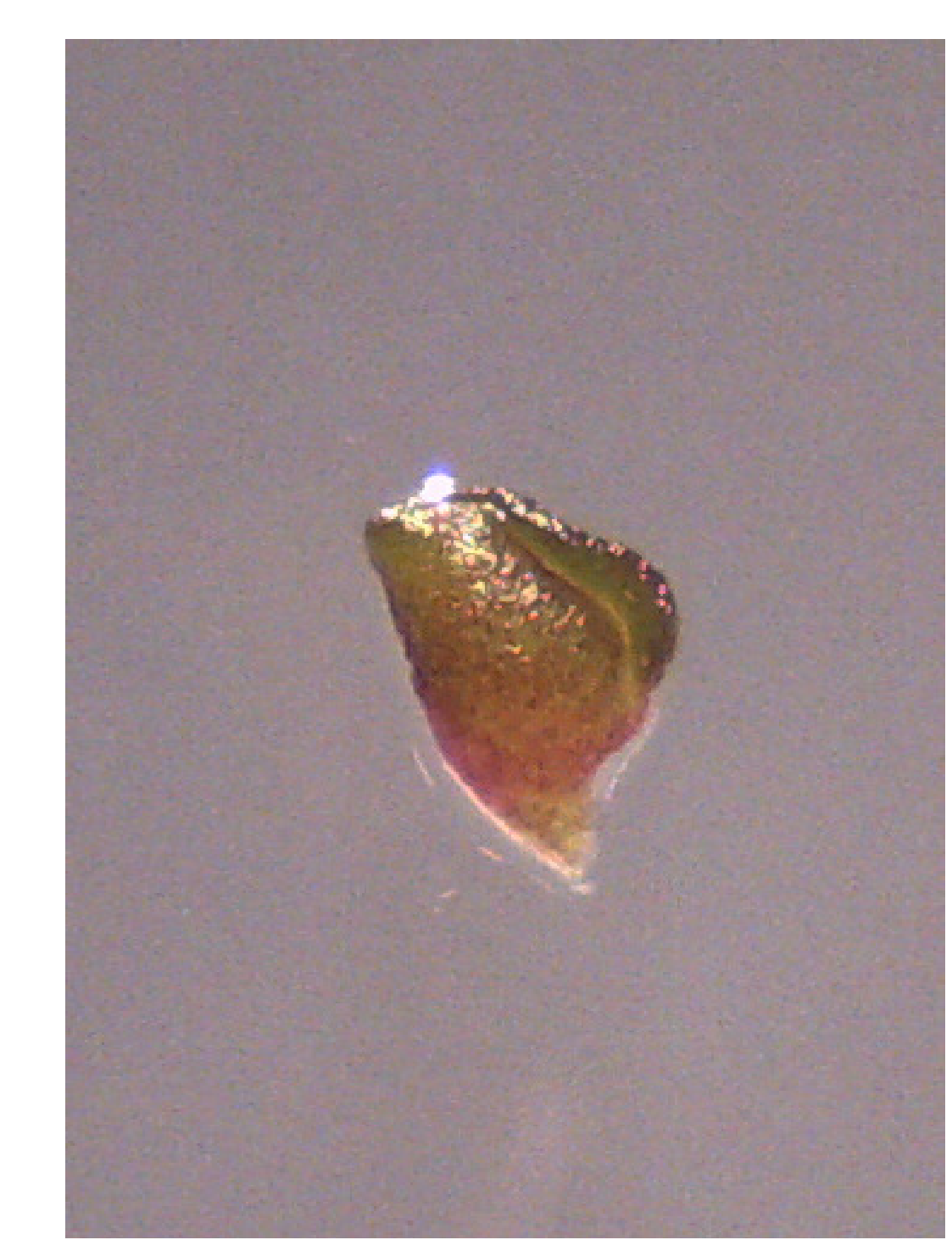

$g n^{s}$

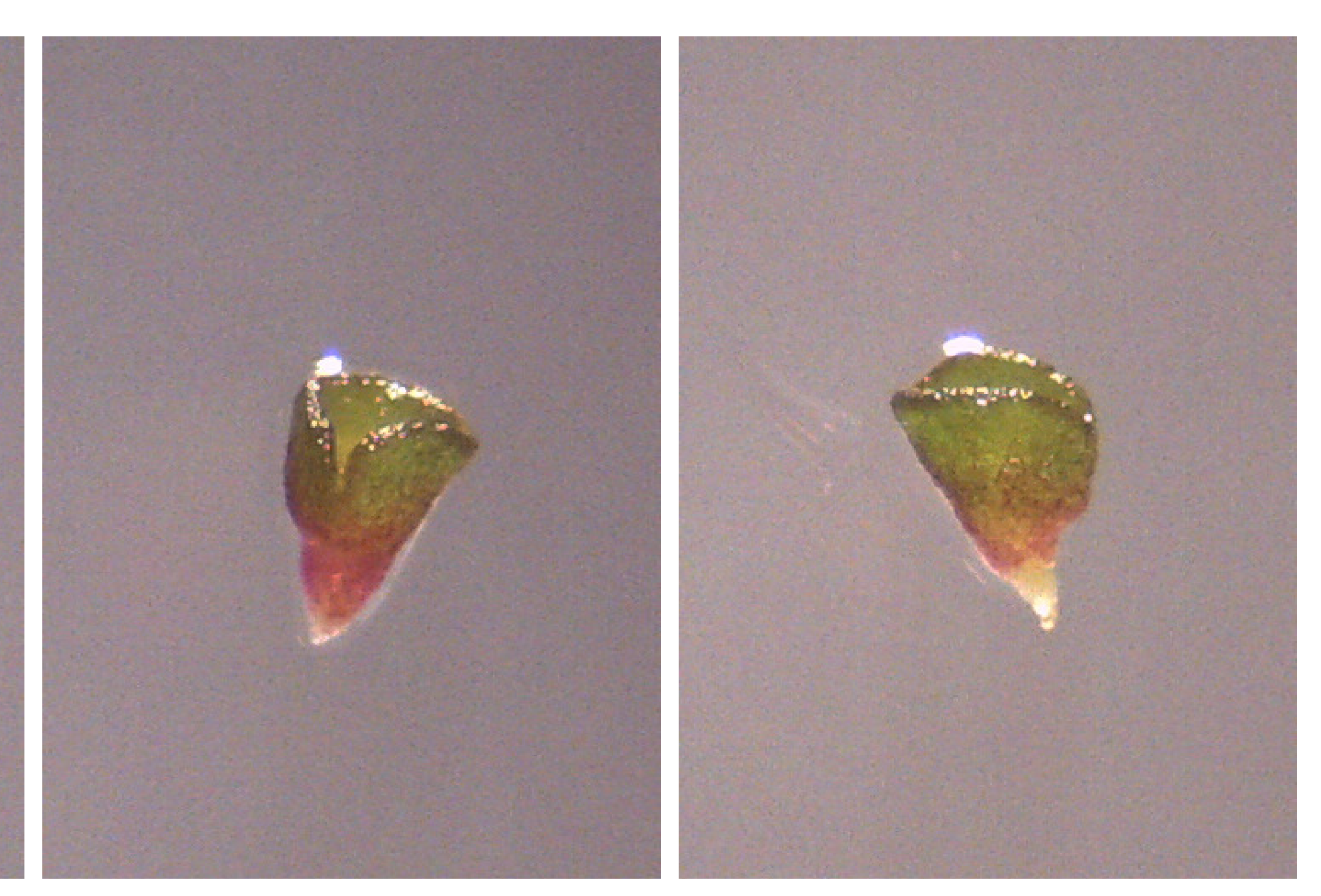

$\mathrm{L} 1$
$g n^{s} \mathrm{GN}_{\text {pro }}:$ GNL1-GFP

D

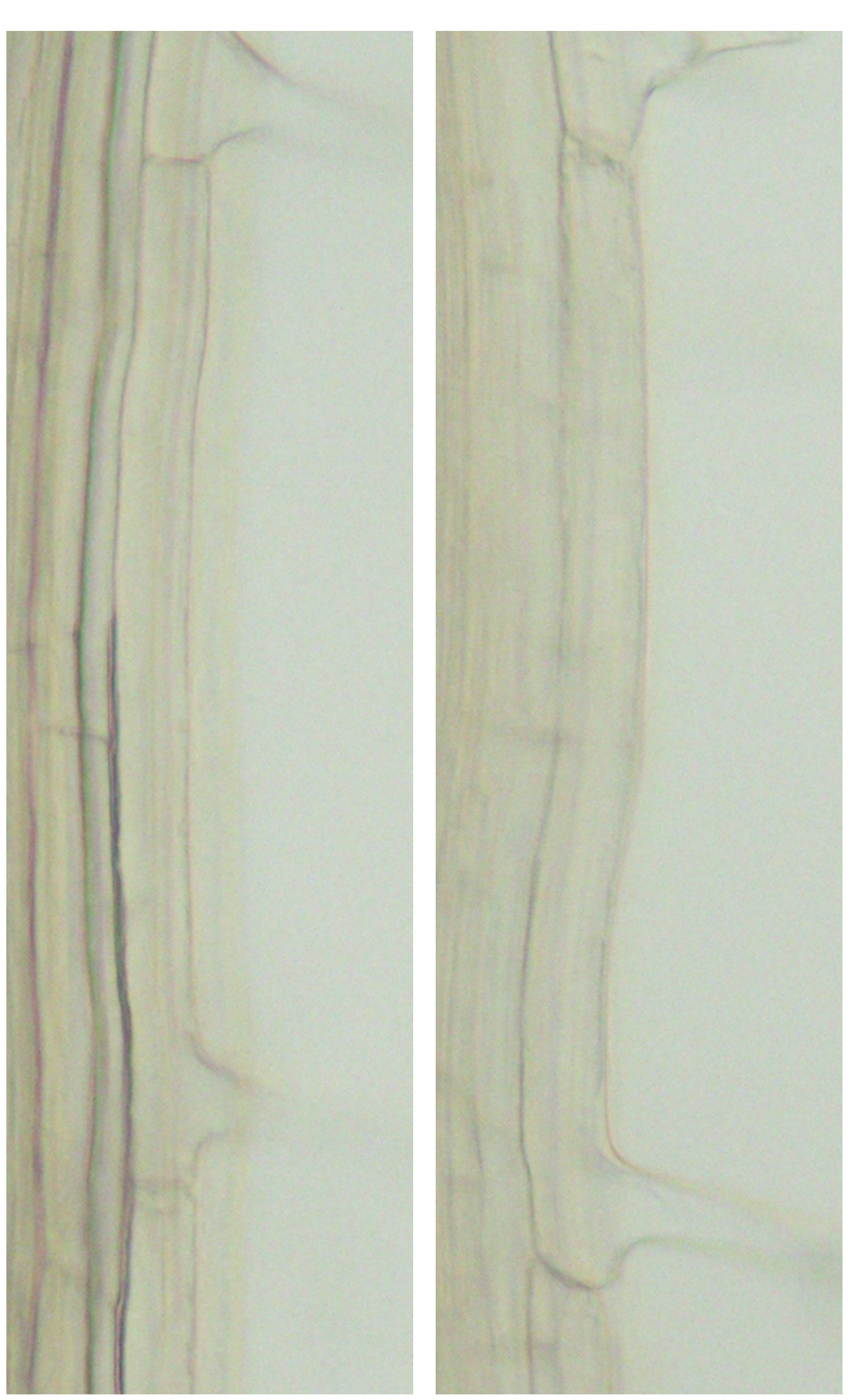

Col-0
B

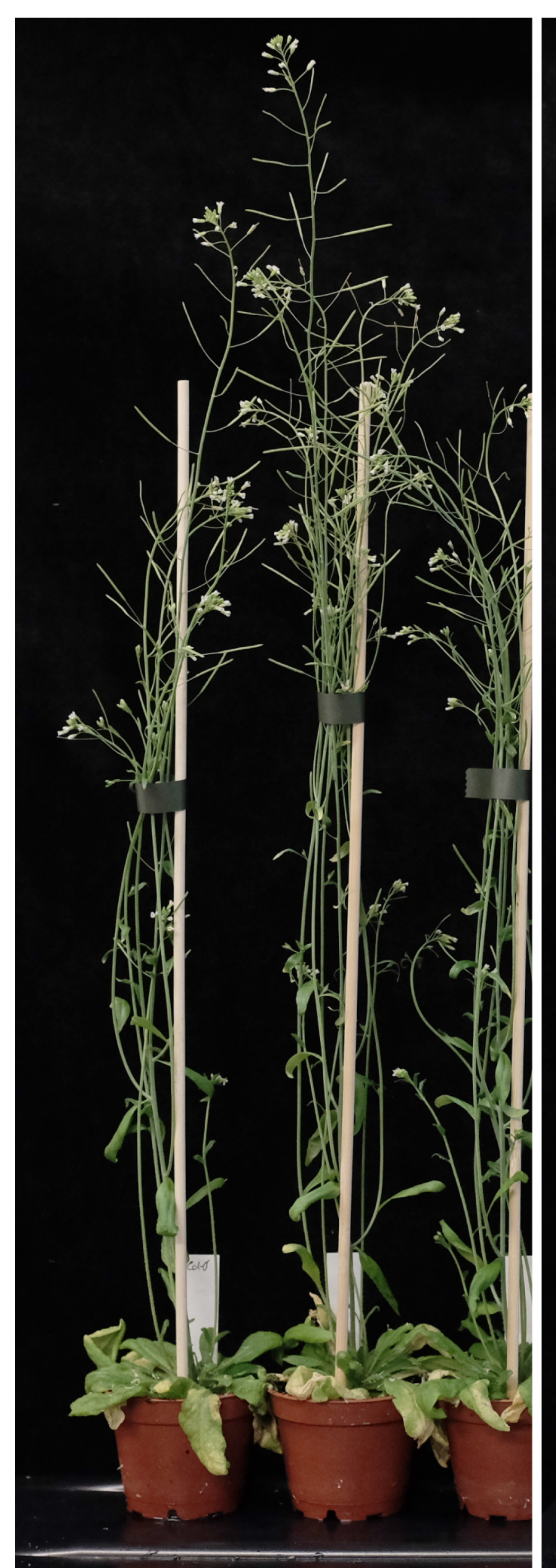

Col-0

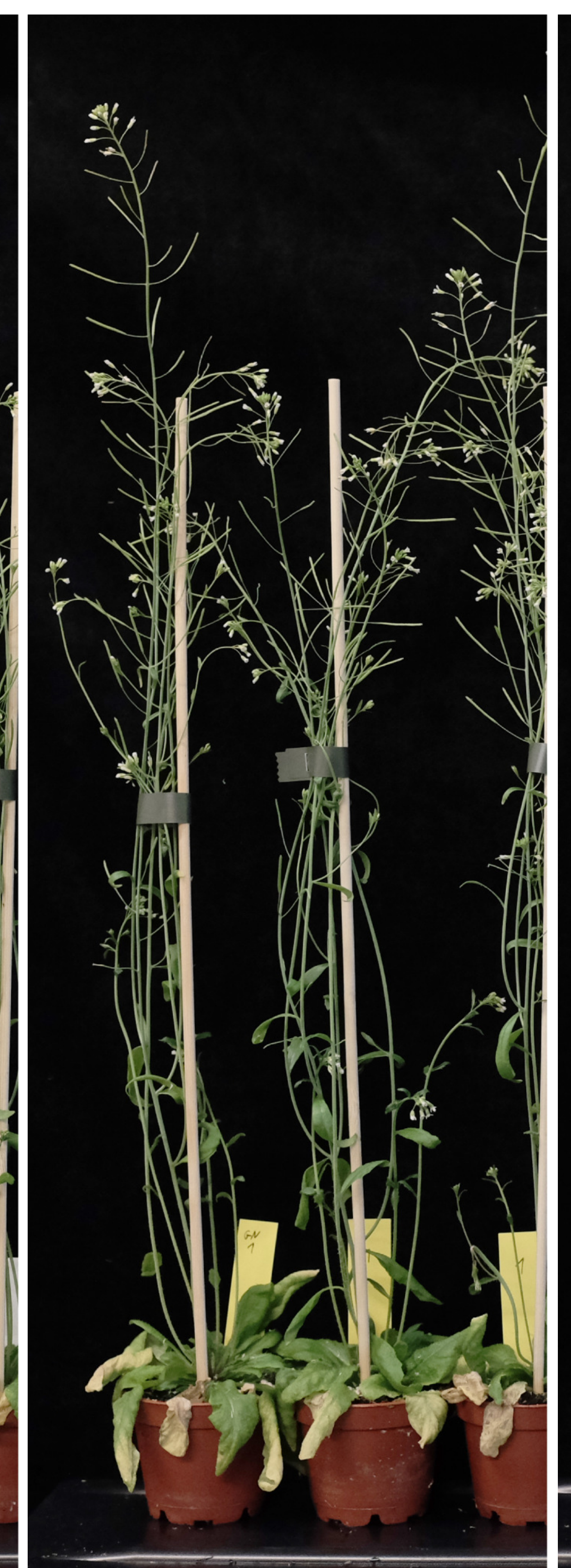

L1

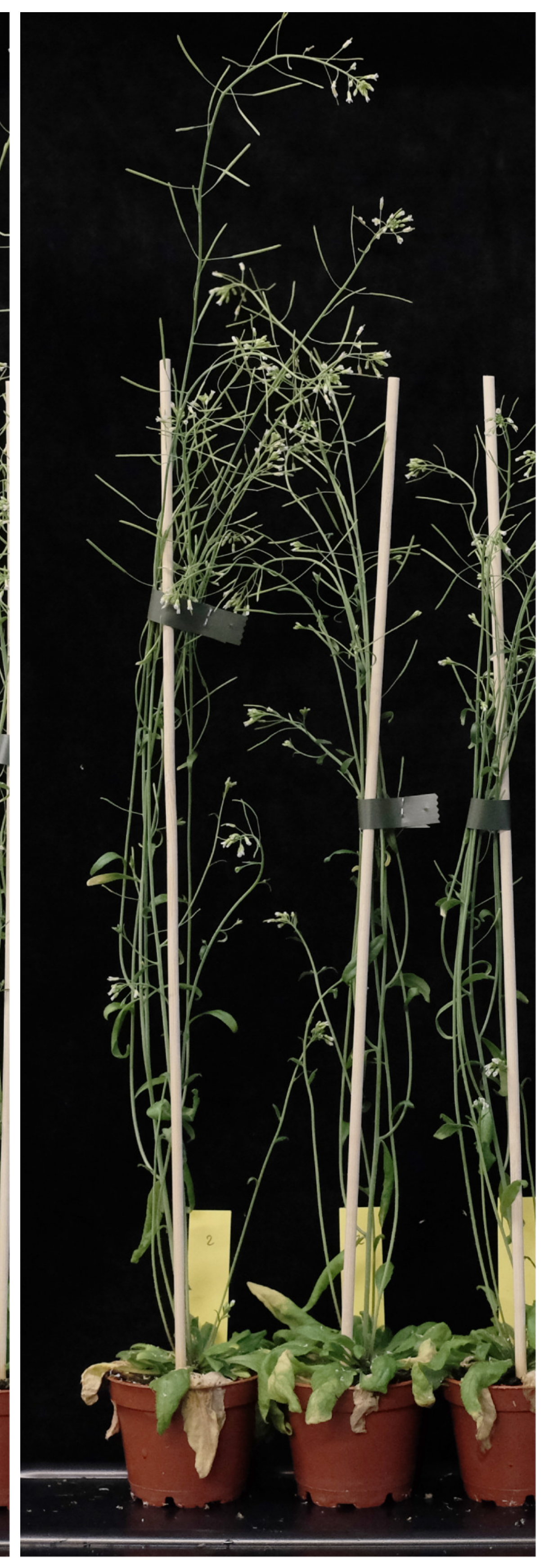

$g n^{s} G N_{p r o}: G N-G F P$
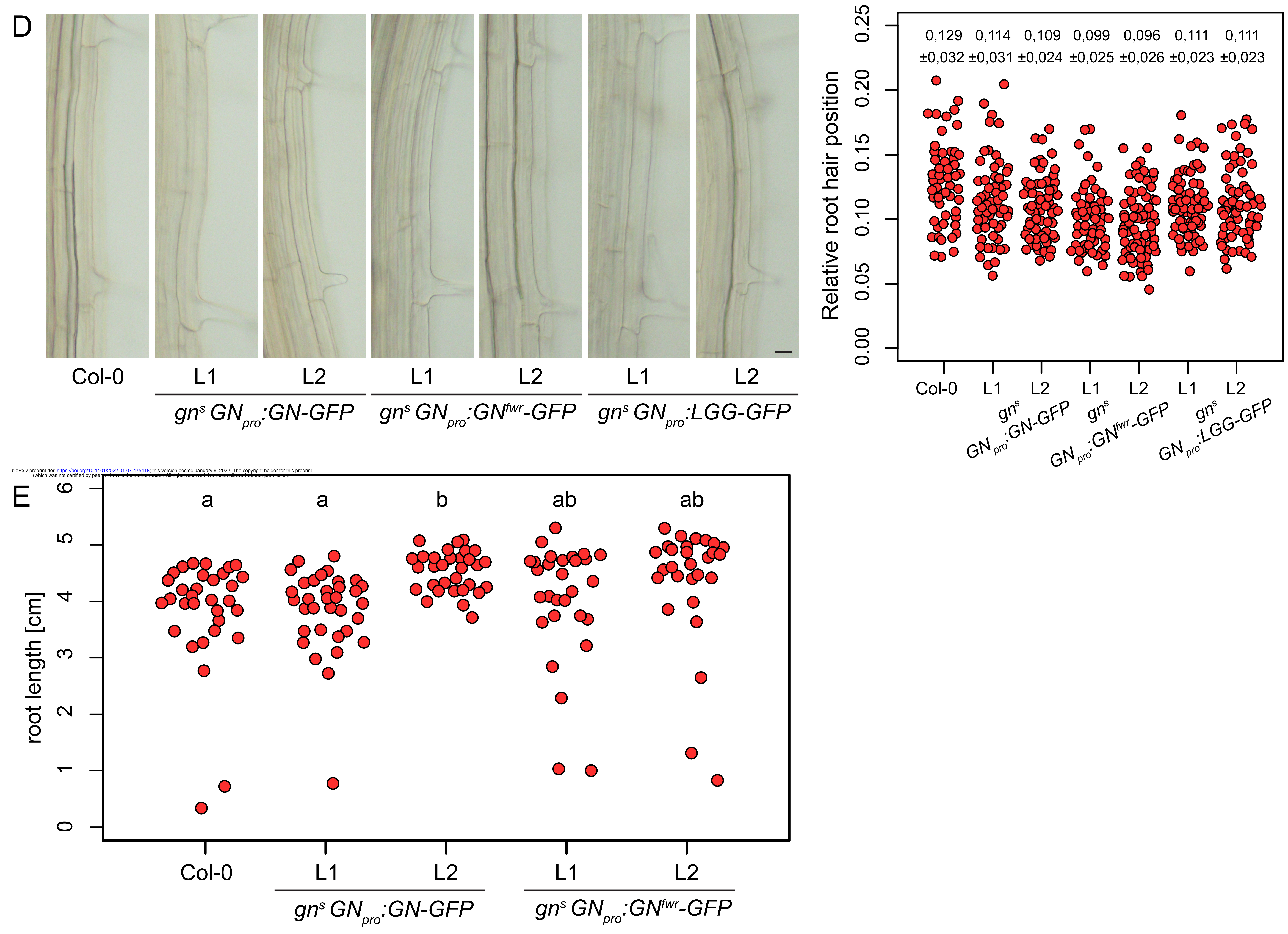
Figure 1-figure supplement 2

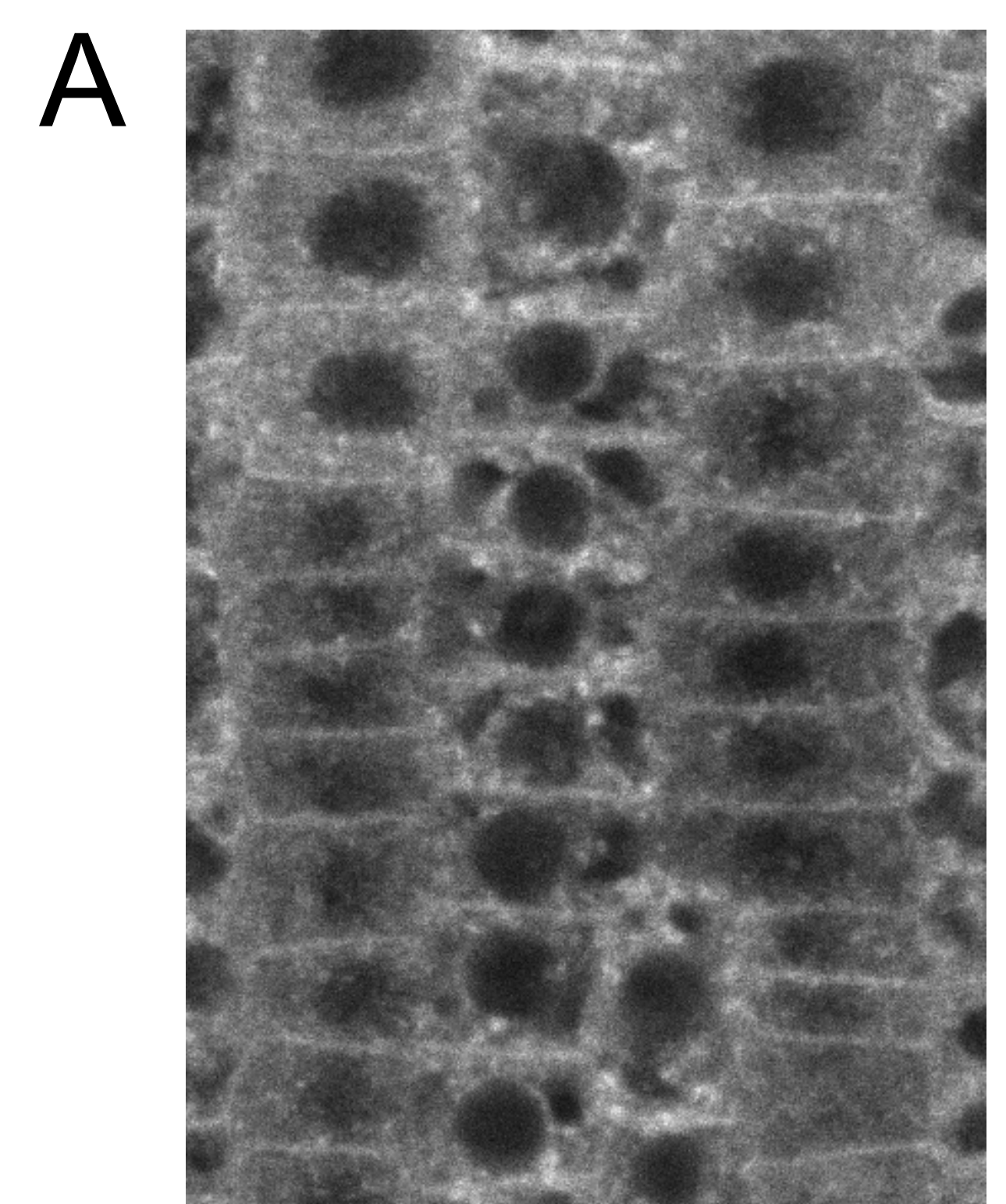

L1
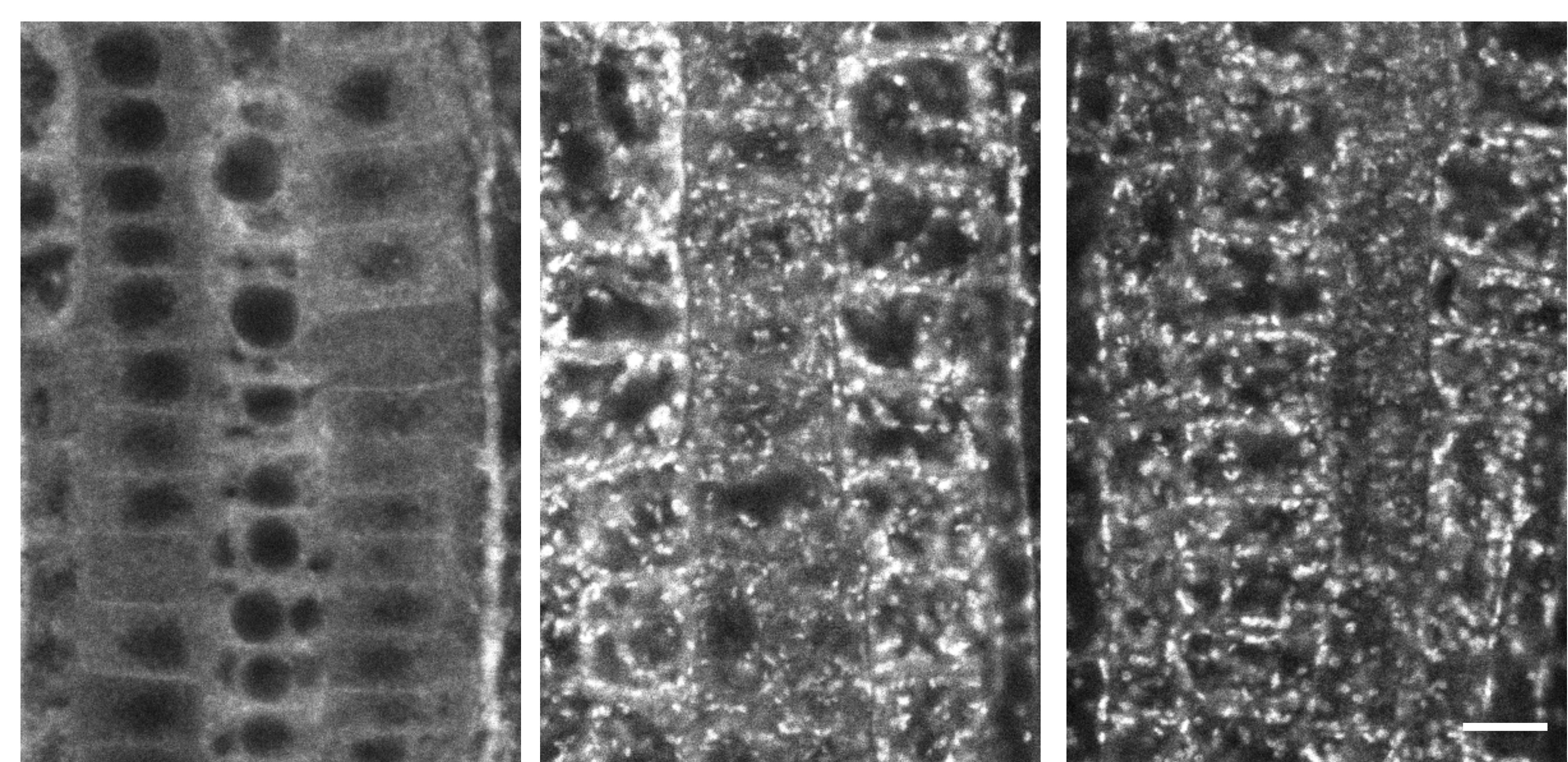

L2

\begin{abstract}
L1
\end{abstract}
$G N_{\text {pro }}: G N L 1-G F P$

B

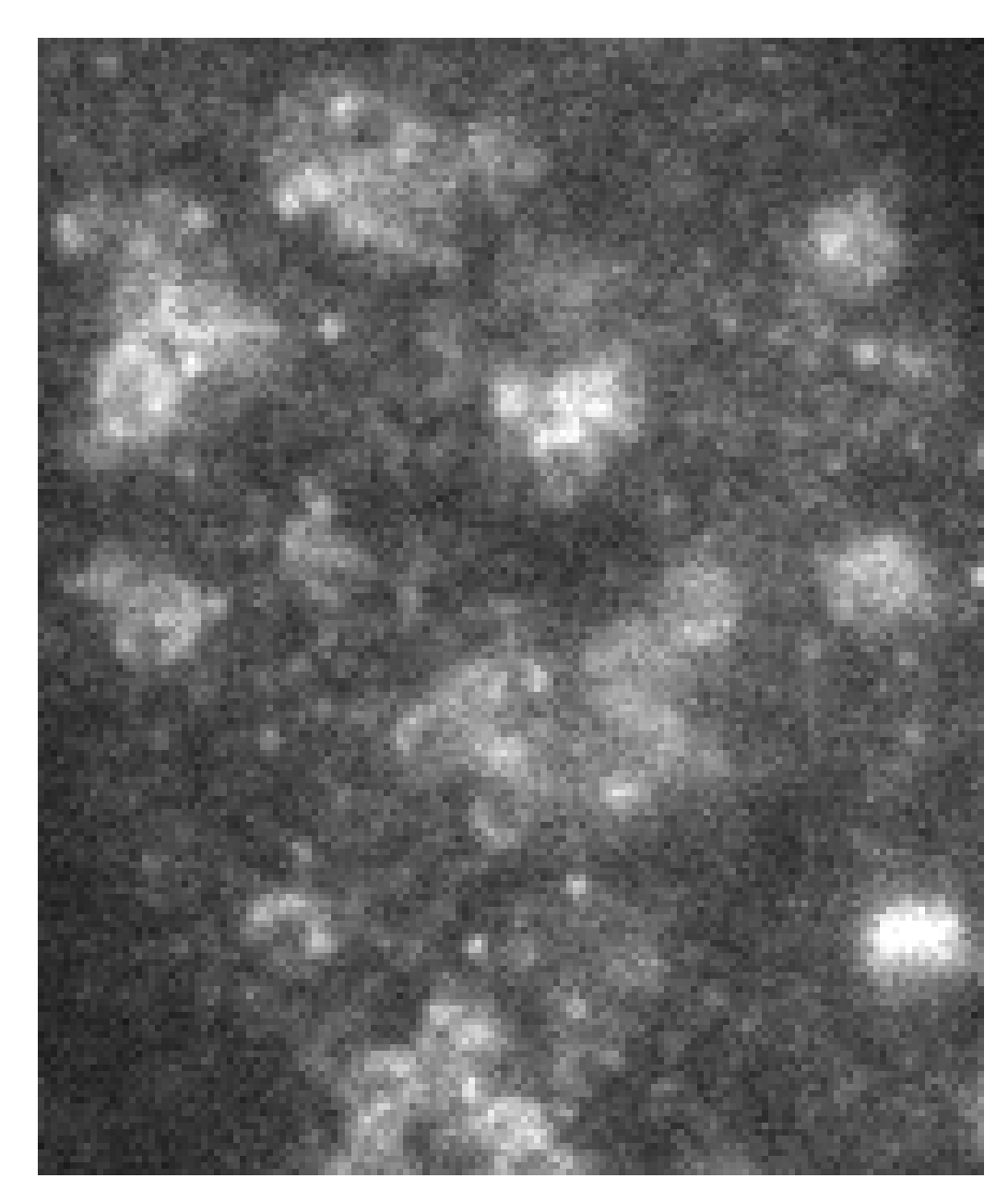

L1

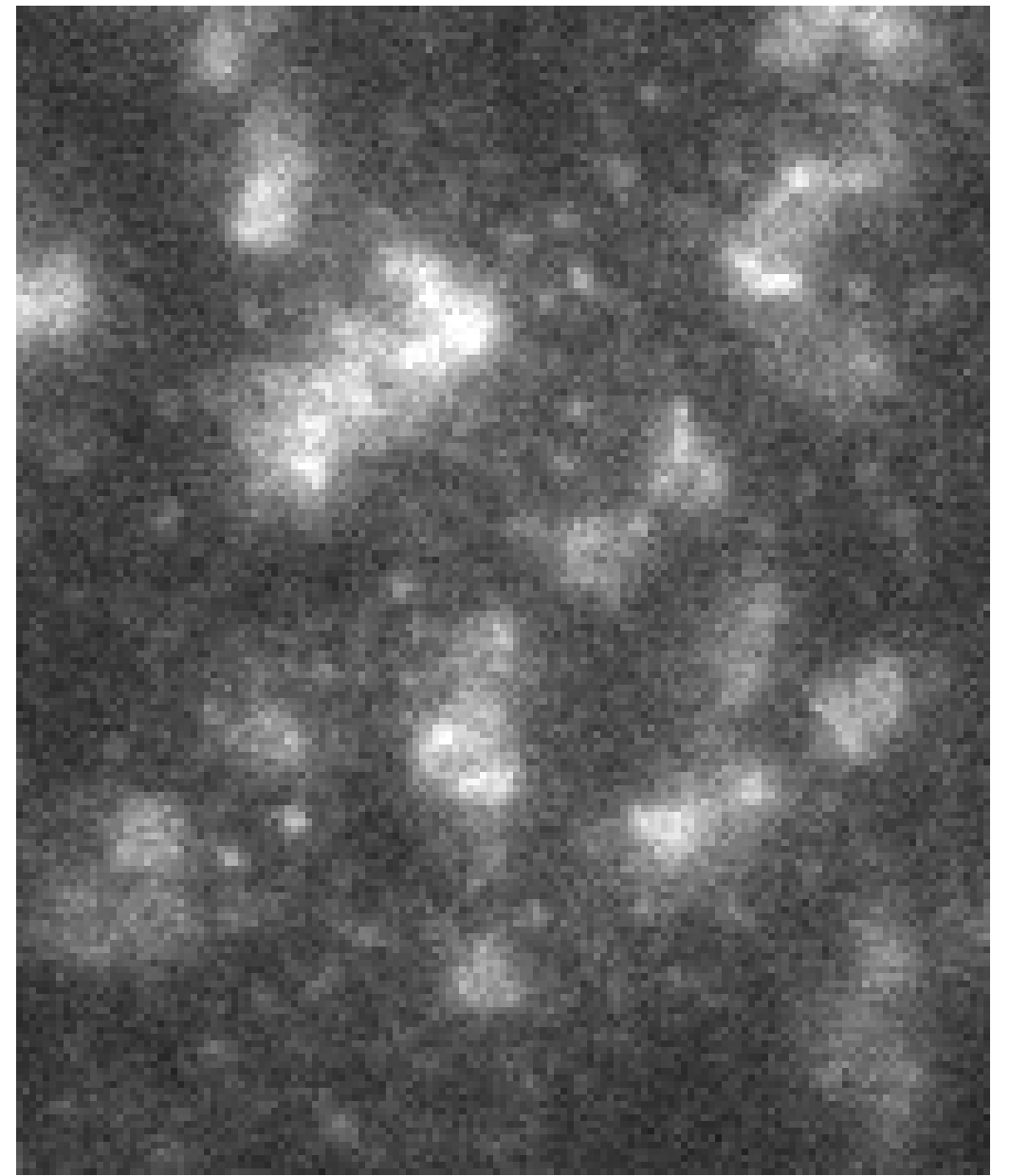

L2
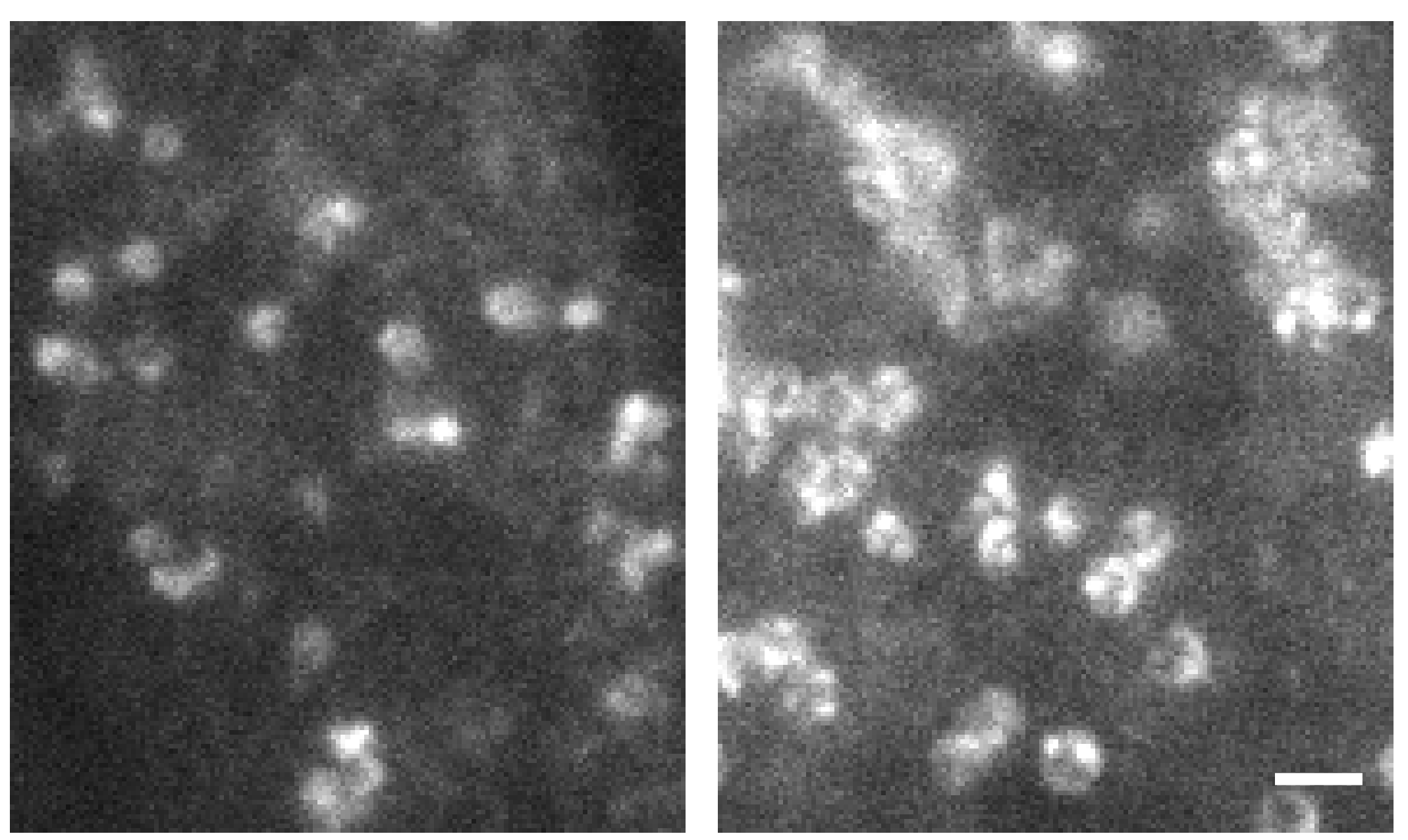

L

$G N_{p r o}: G N-G F P$

$G_{\text {pro }}: G N L 1-G F P$ 
Figure 1-figure supplement 3

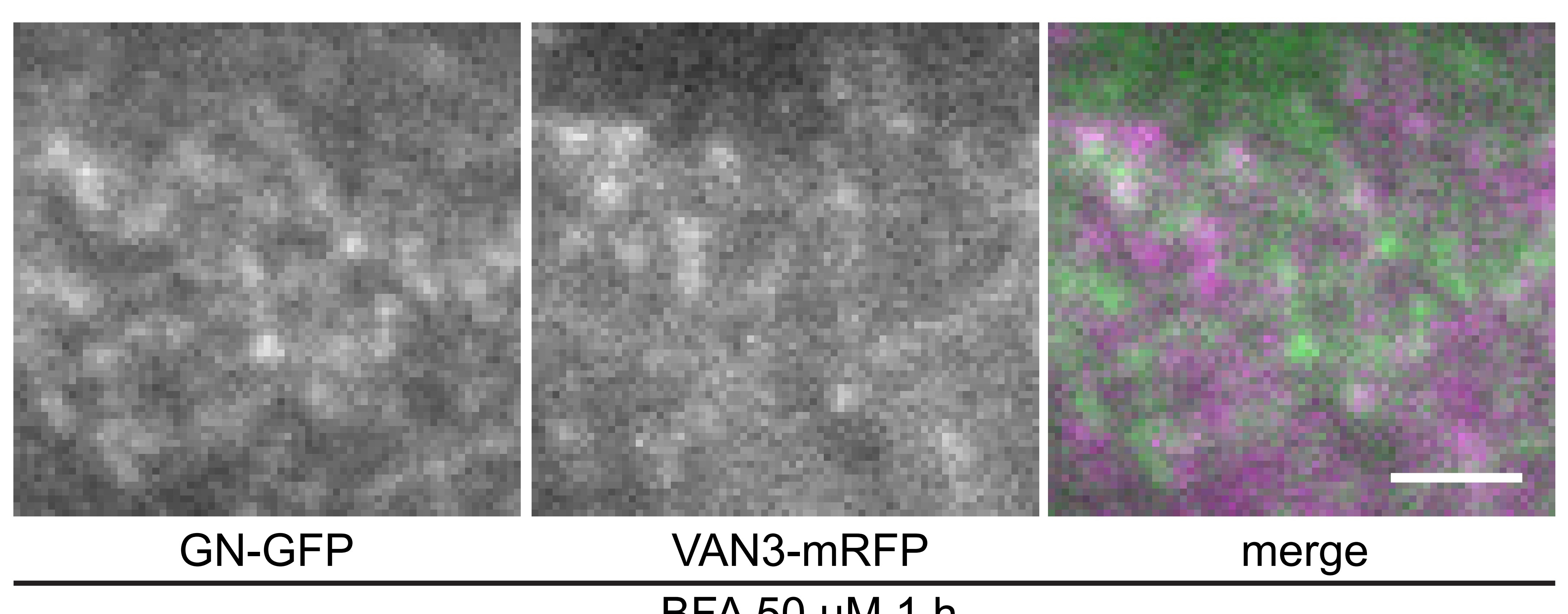

BFA $50 \mu \mathrm{M} 1 \mathrm{~h}$ 
Figure 1-figure supplement 4
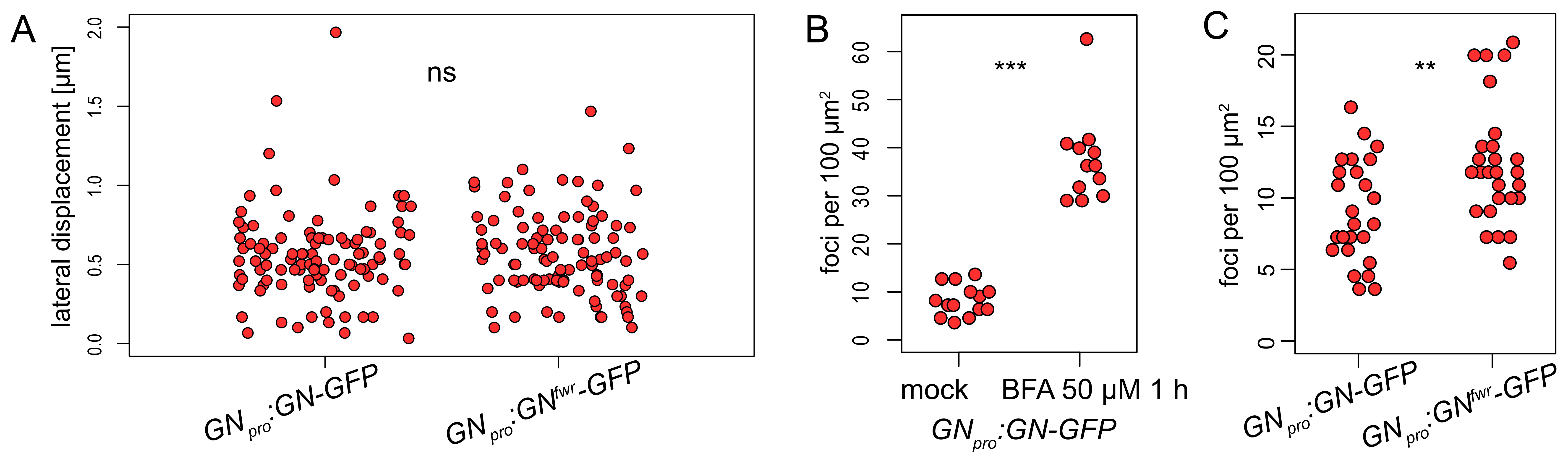


\section{Figure 2}

A

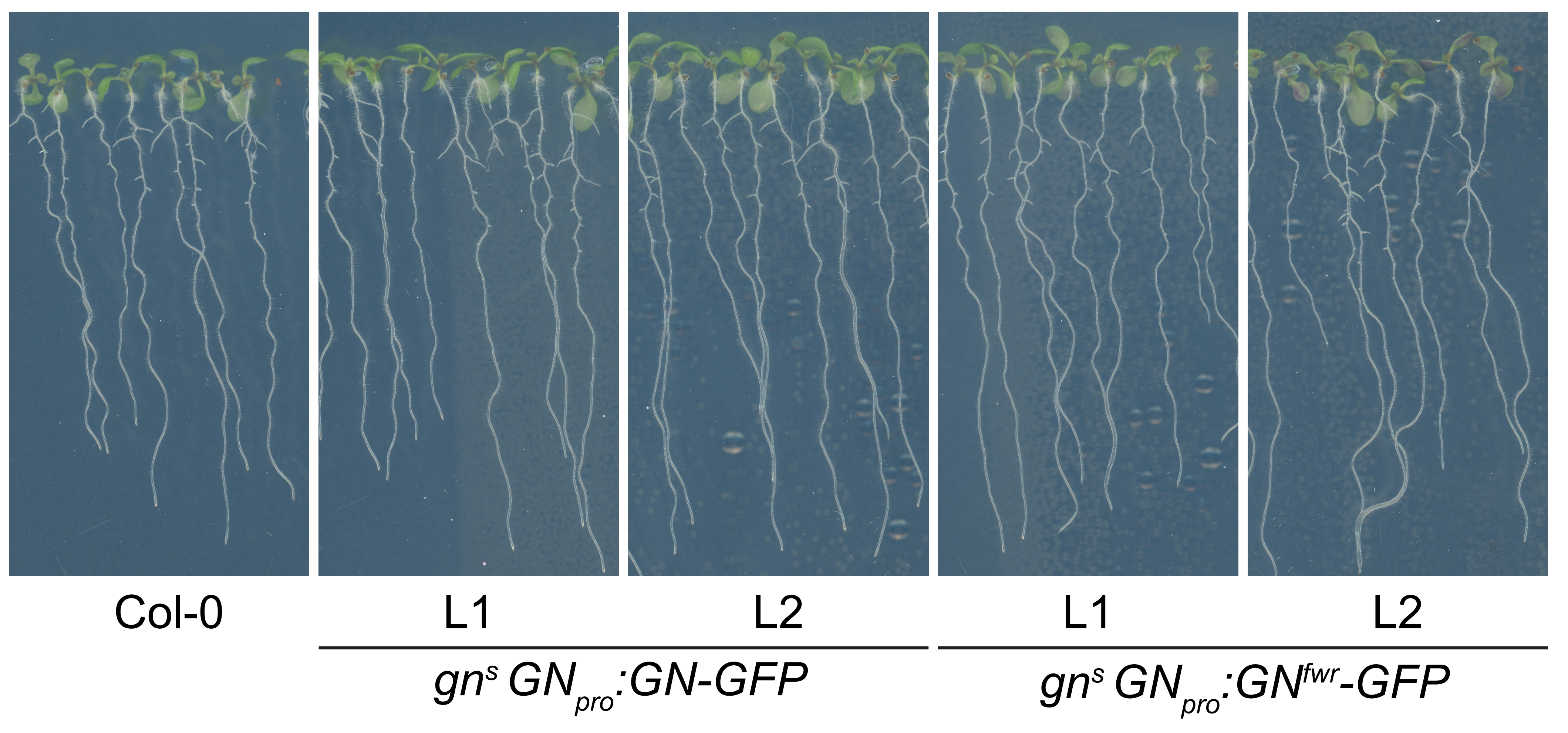

C

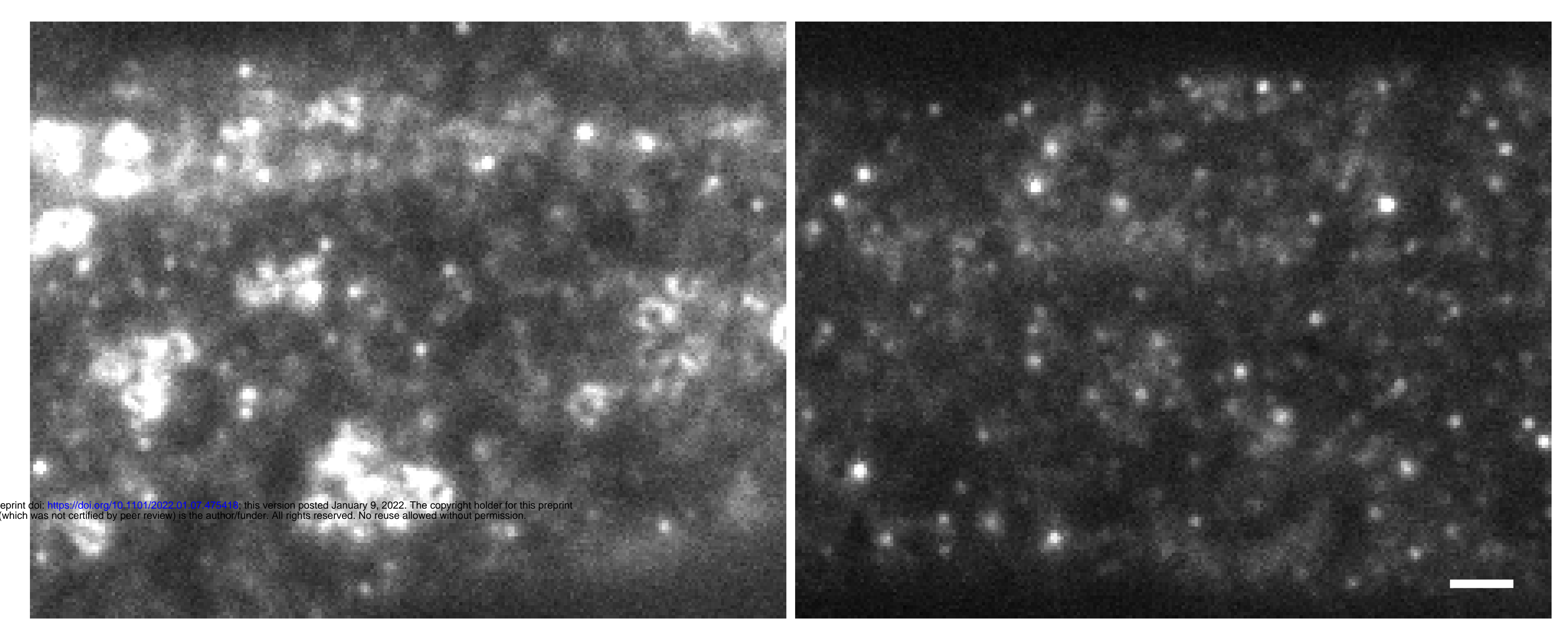

$g n^{s} G N_{\text {pro }}: G N-G F P$

D

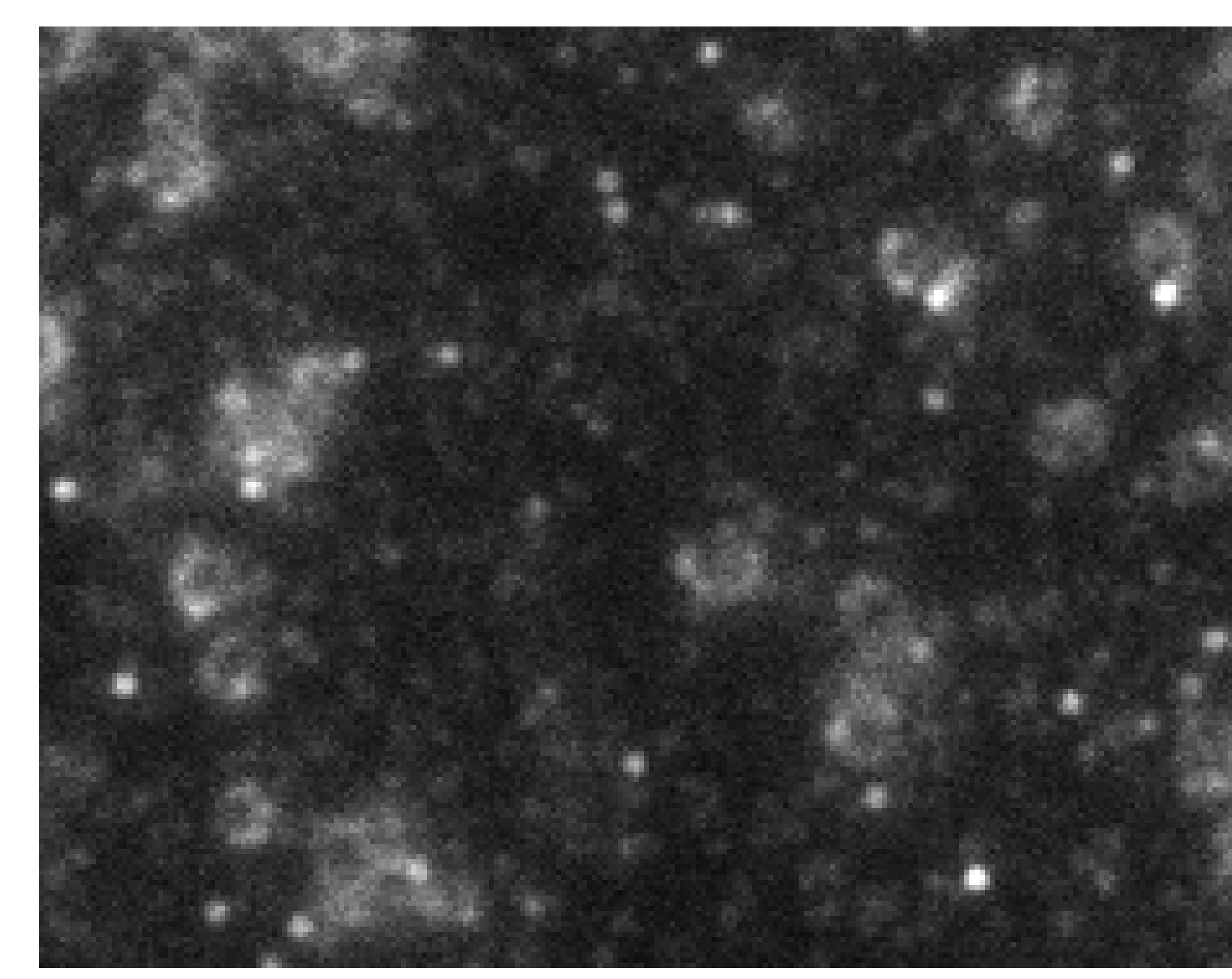

$g n^{s} \mathrm{GN}_{\text {pro }}: G N-G F P$ $g n^{s} G N_{p r o}: G N^{f w r}-G F P$

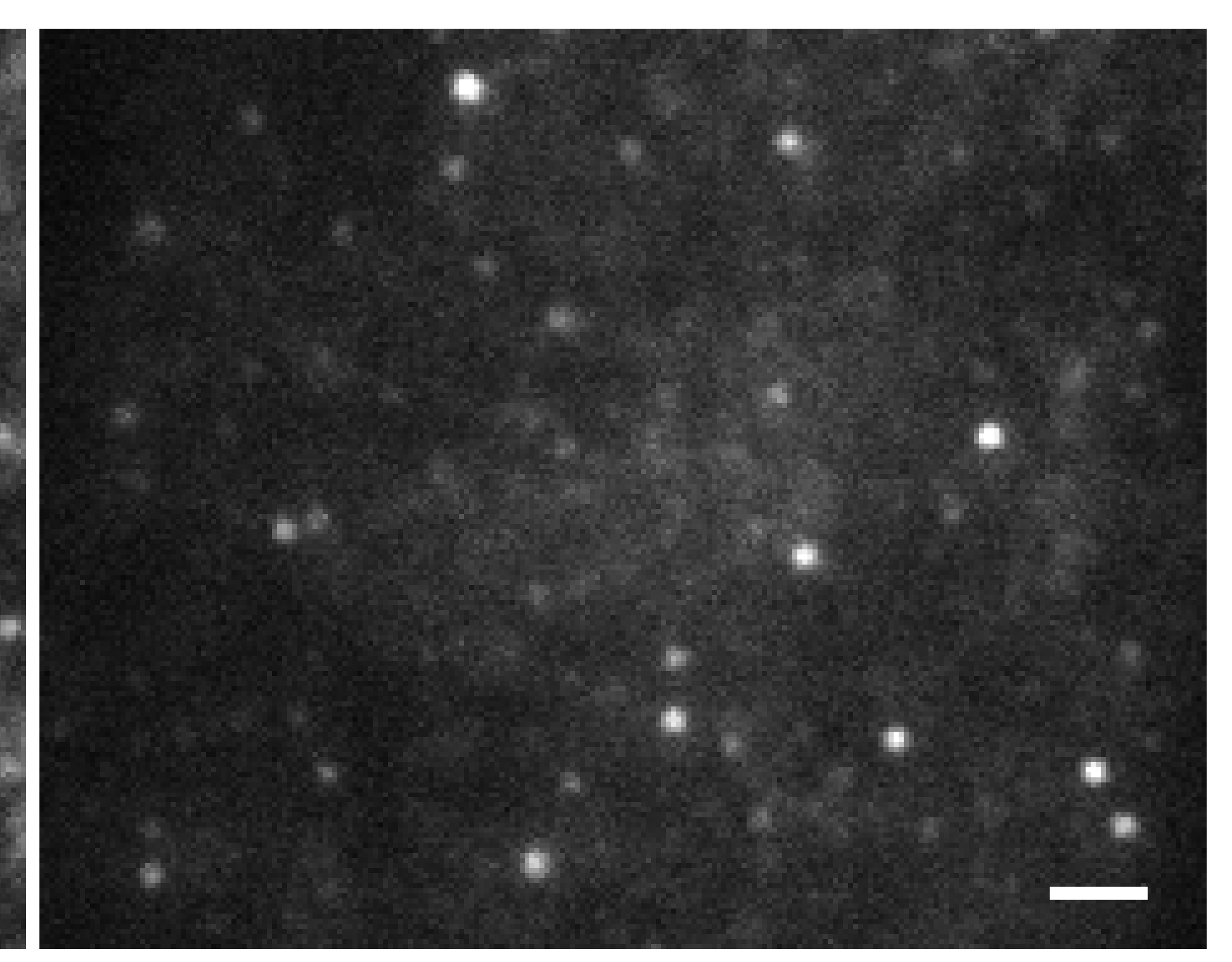

$g n^{s} G N_{\text {pro }}: G N^{f w r}-G F P$
B

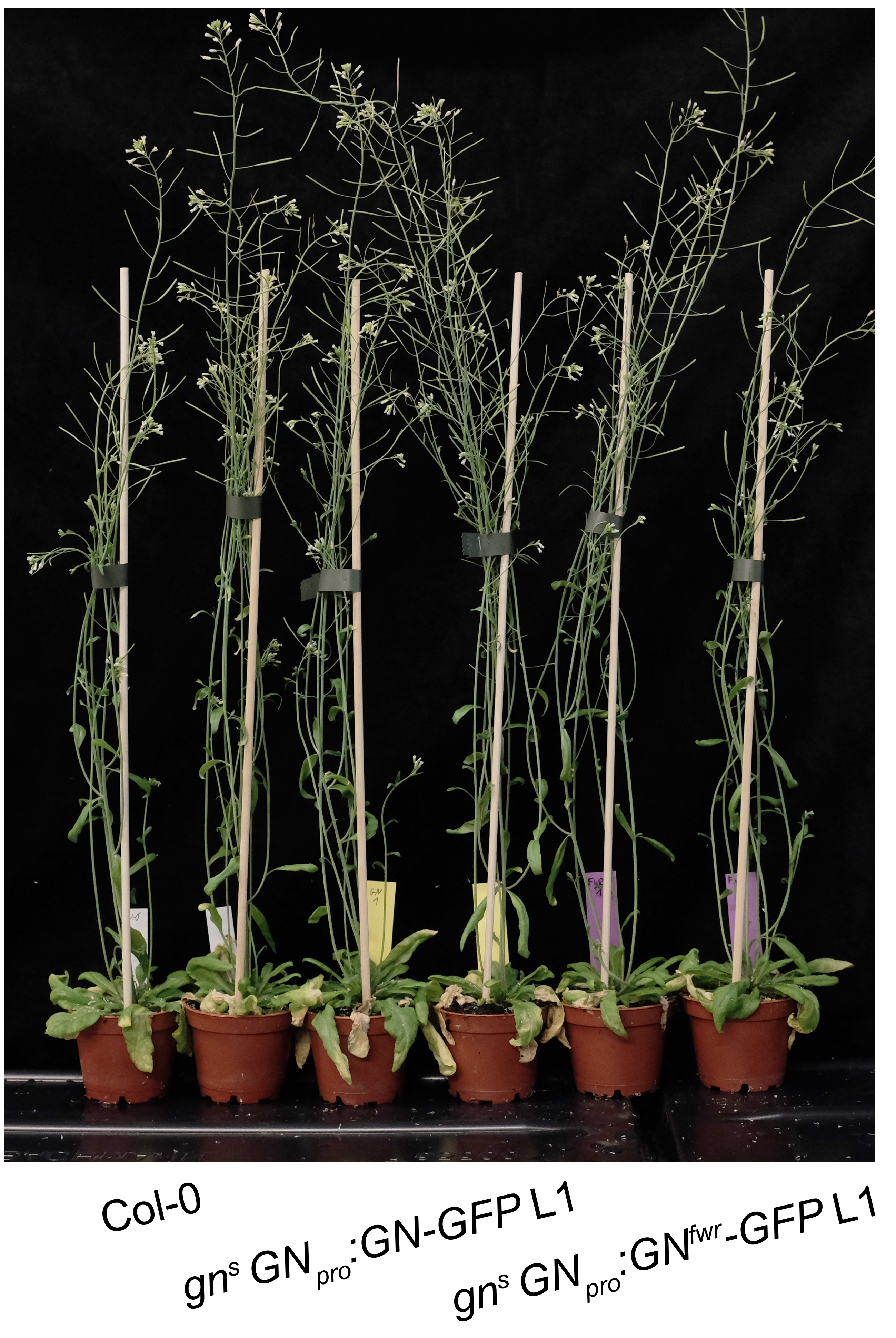

E

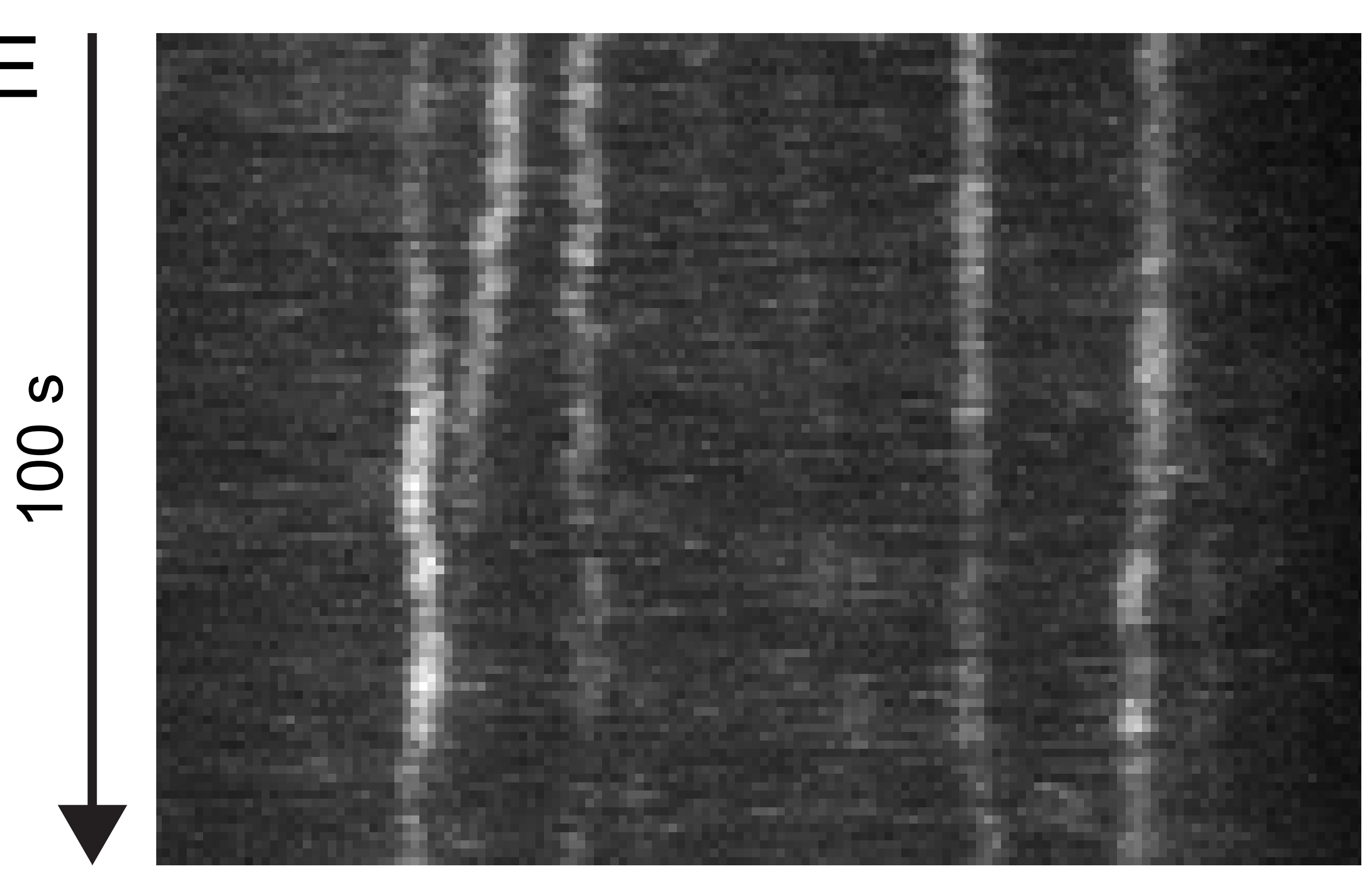

$g n^{s} G N_{p r o}: G N^{f w r}-G F P$ 
Figure 2-figure supplement 1

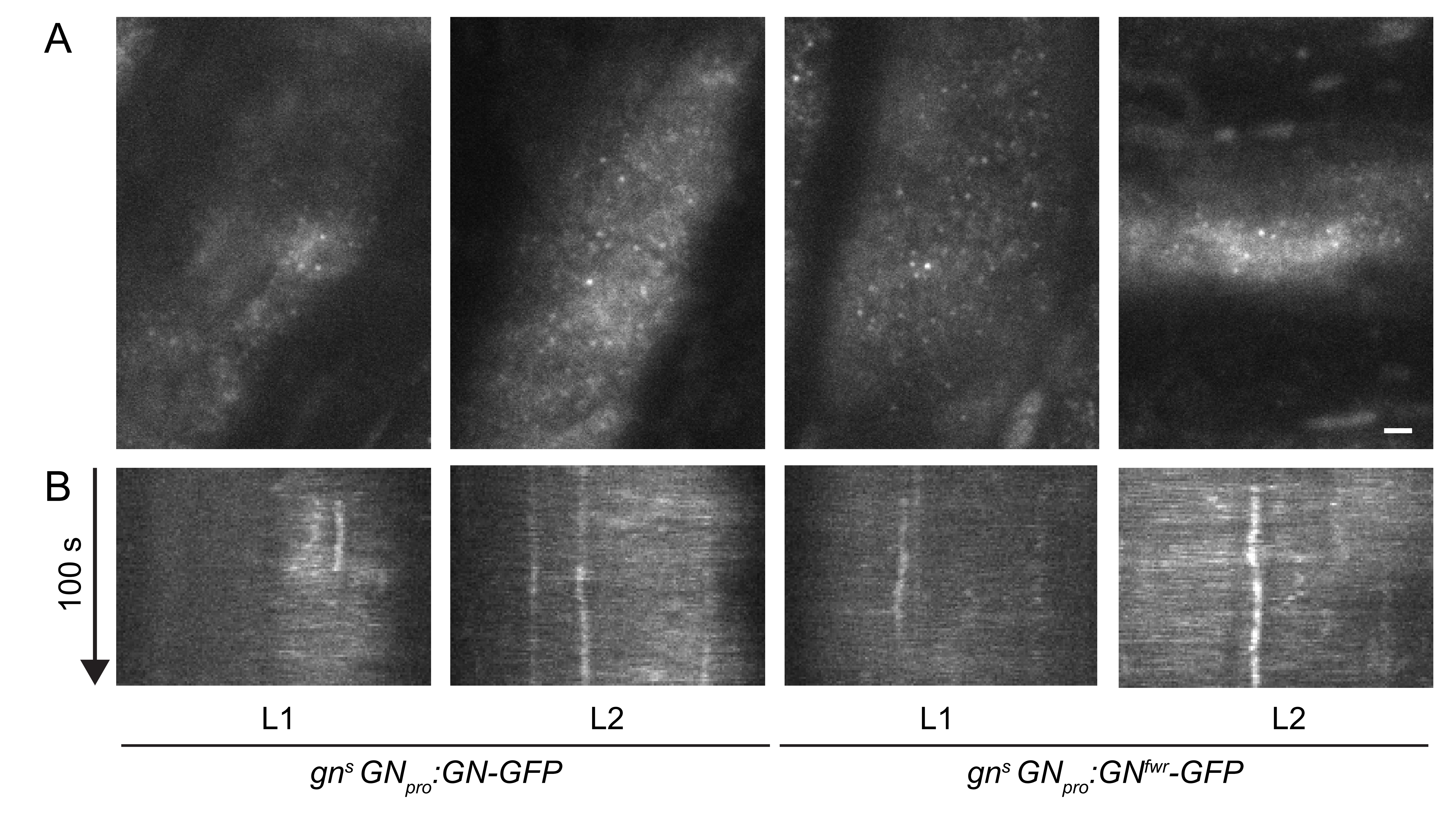




\section{Figure 3}

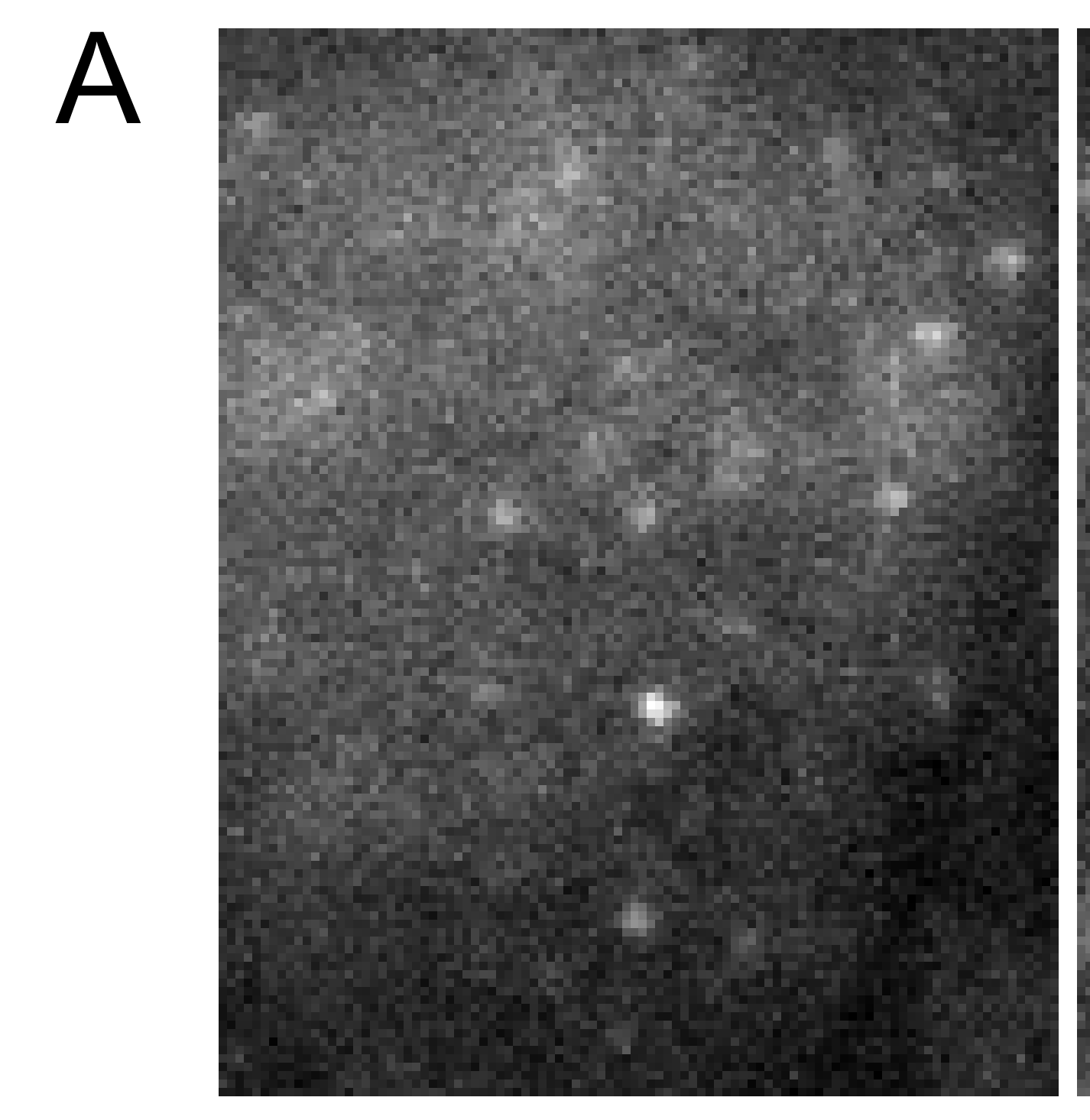

$$
G N_{p r o}: G N-G F P
$$

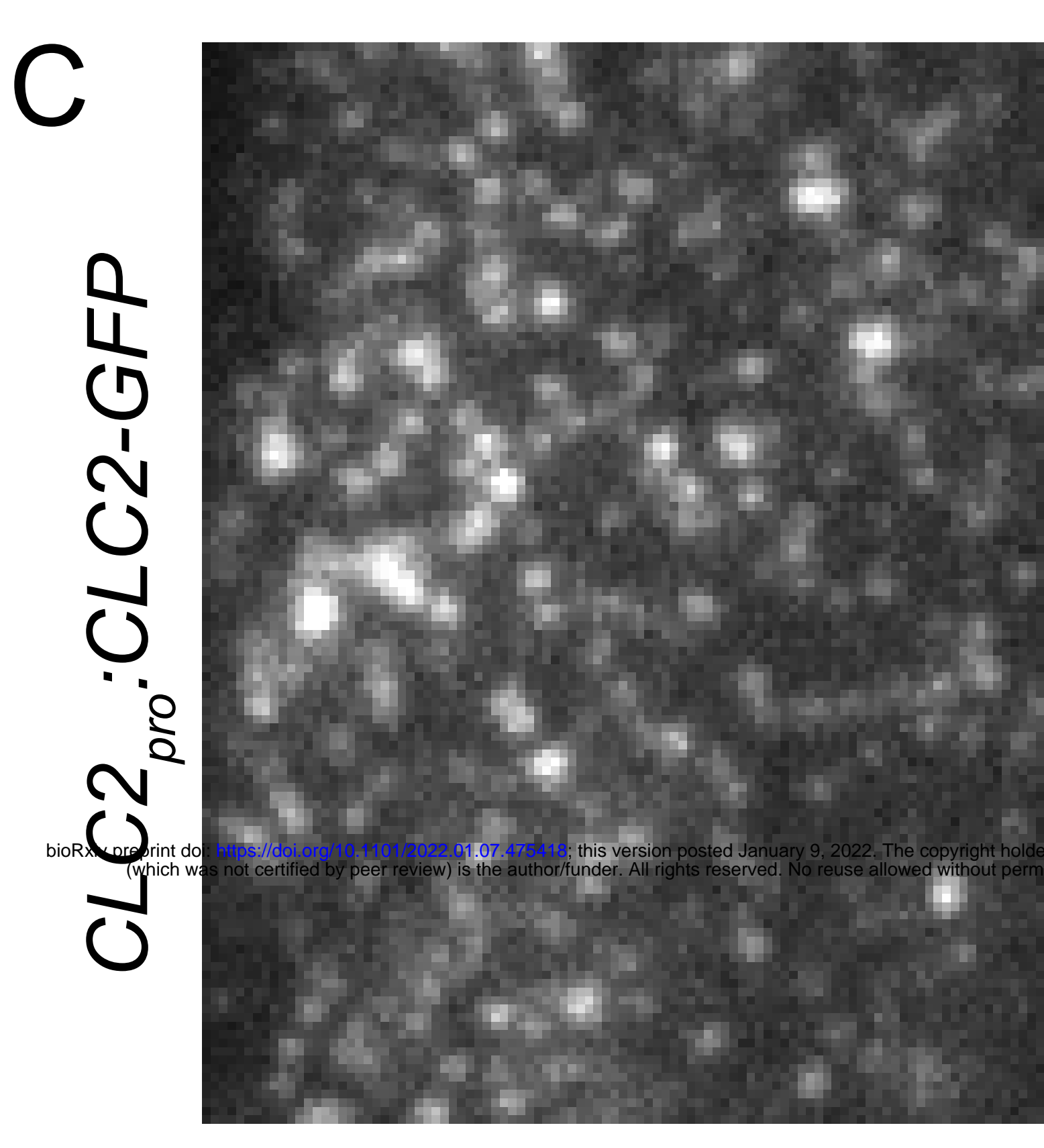

wild type

E

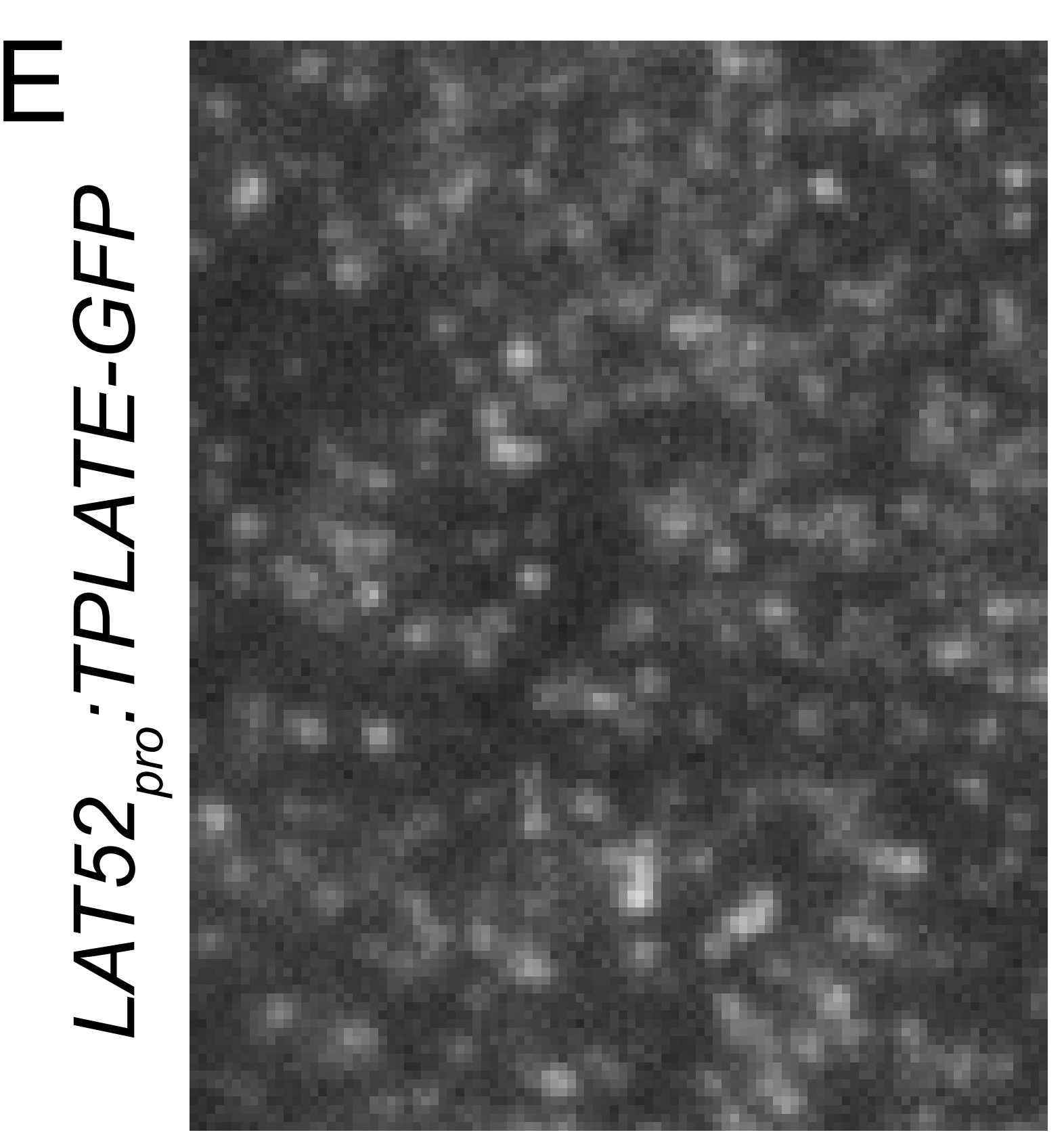

wild type

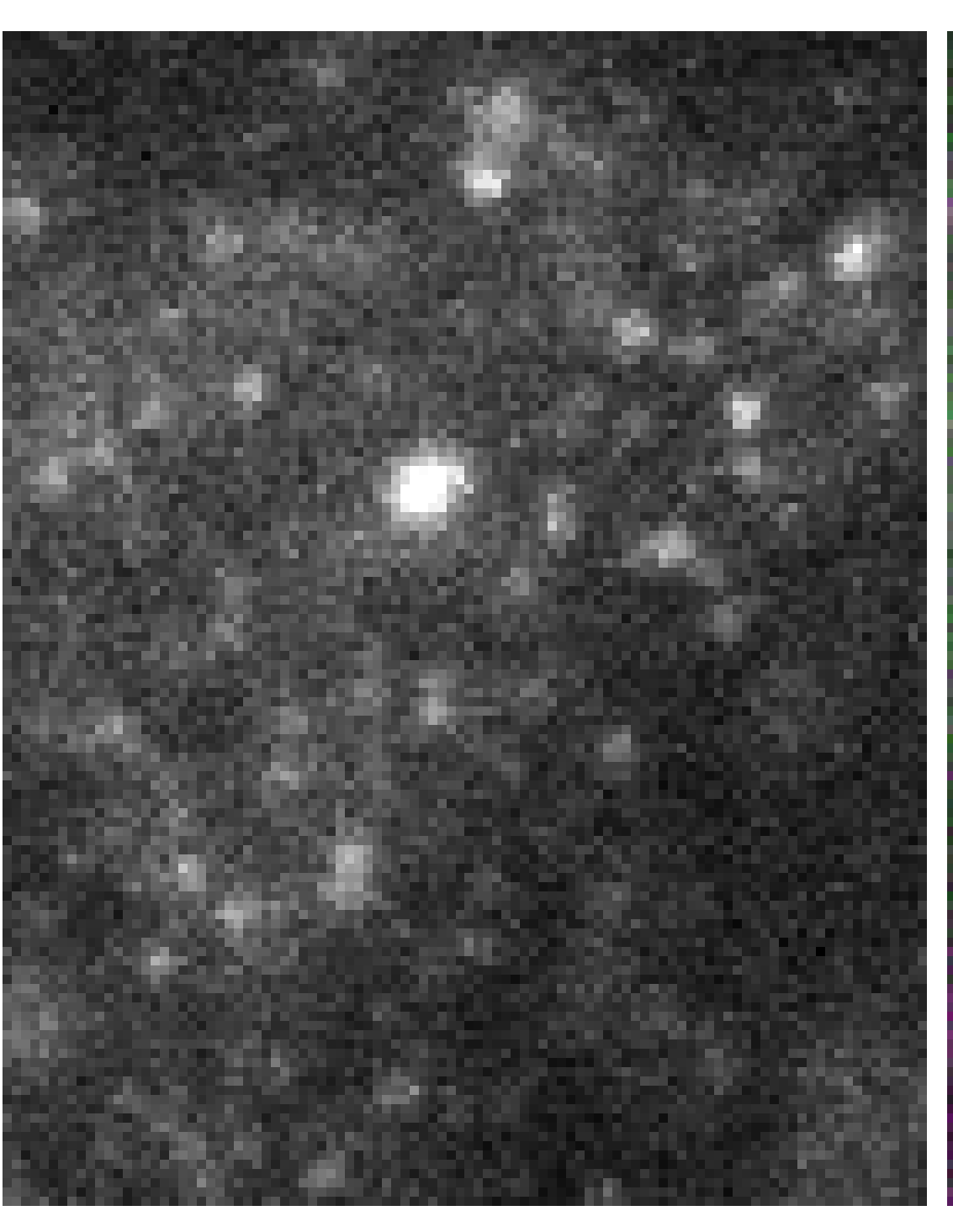

$35 S_{p r o}: C L C-m K O$

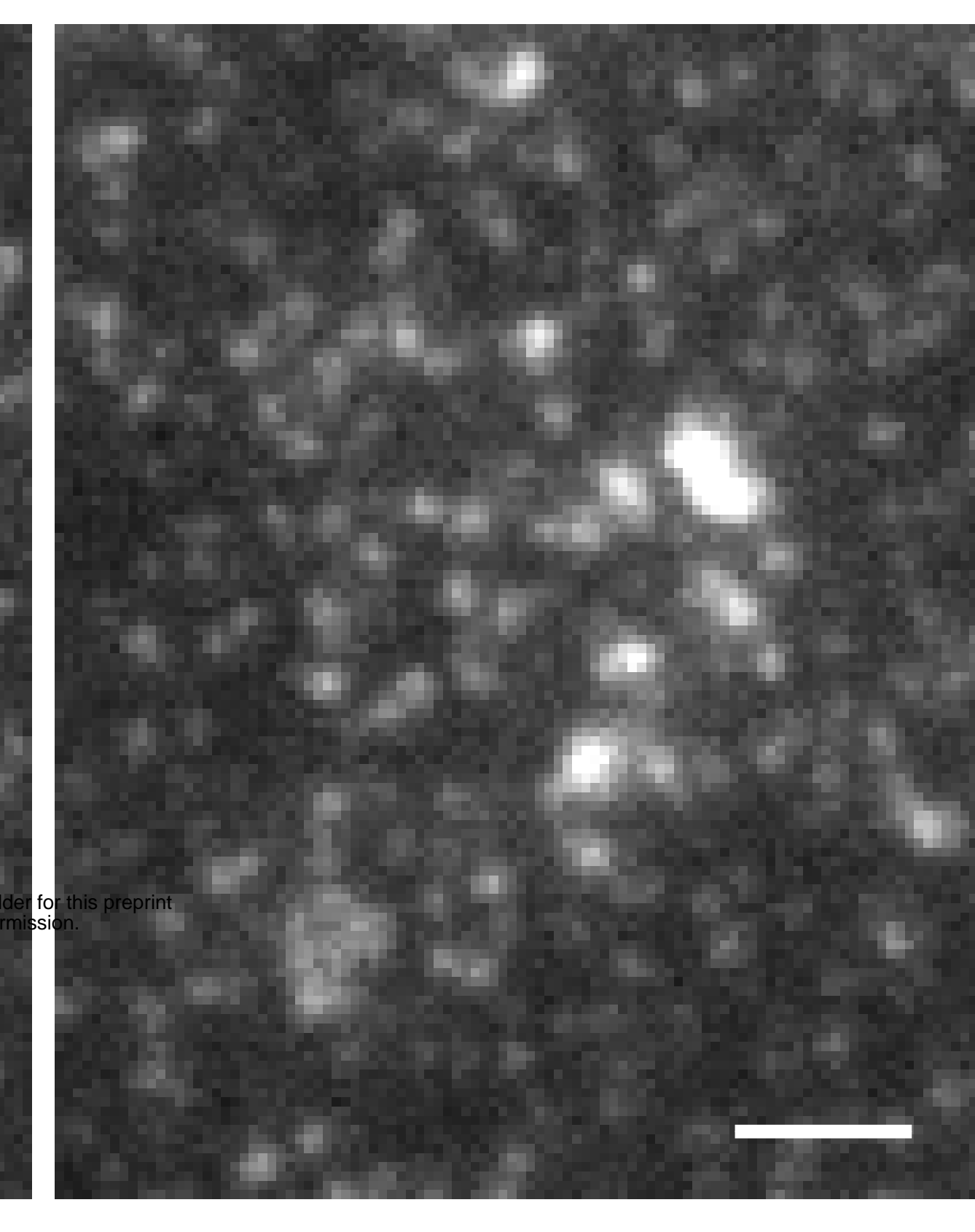

$g n^{s}$

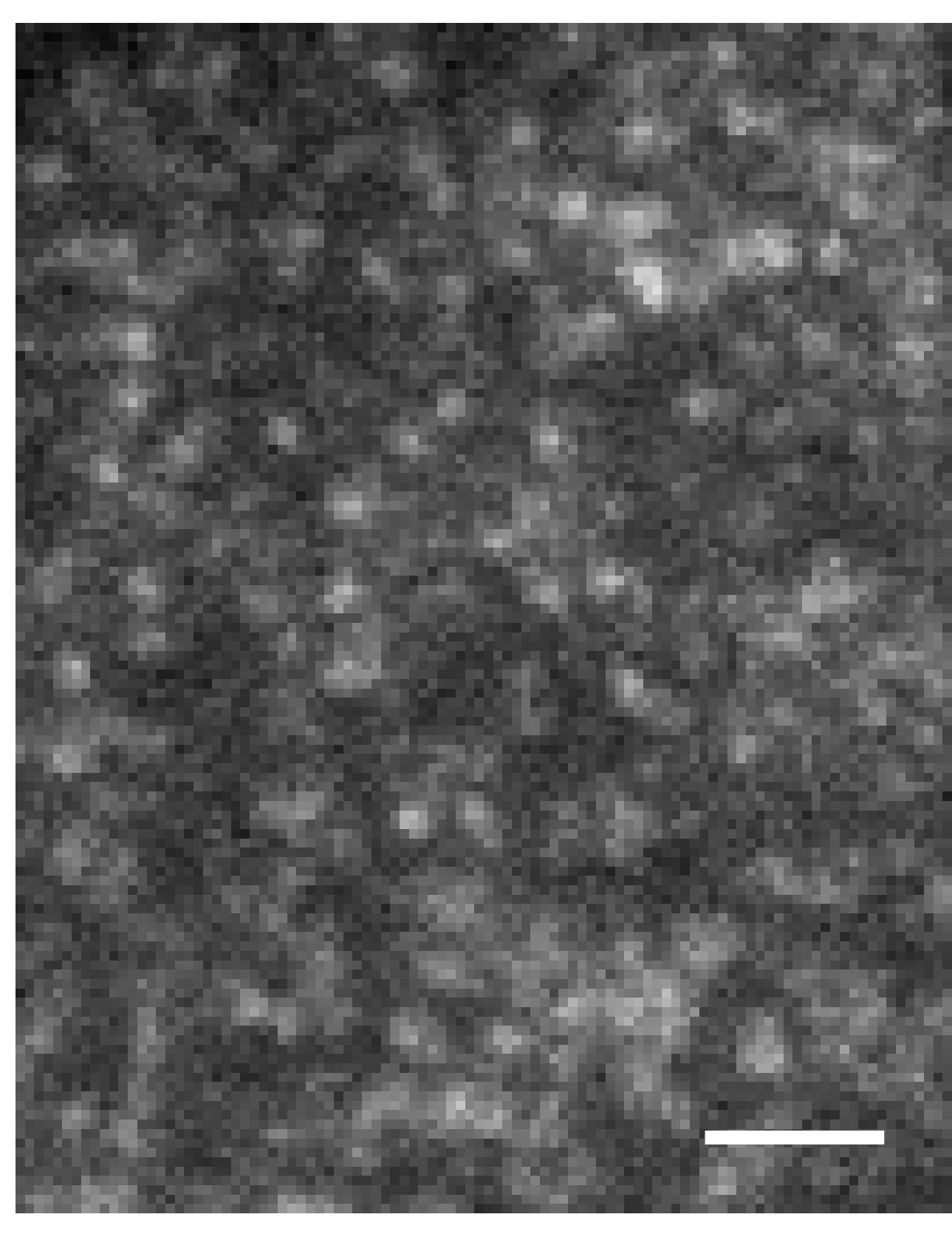

$g n^{s}$
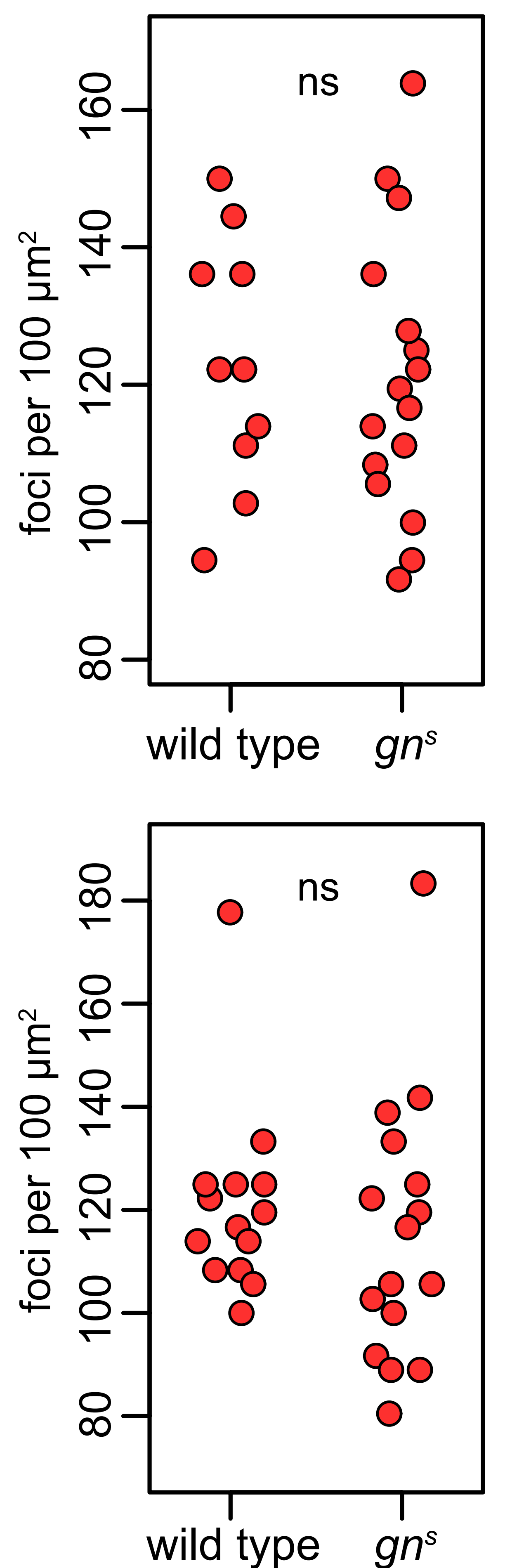

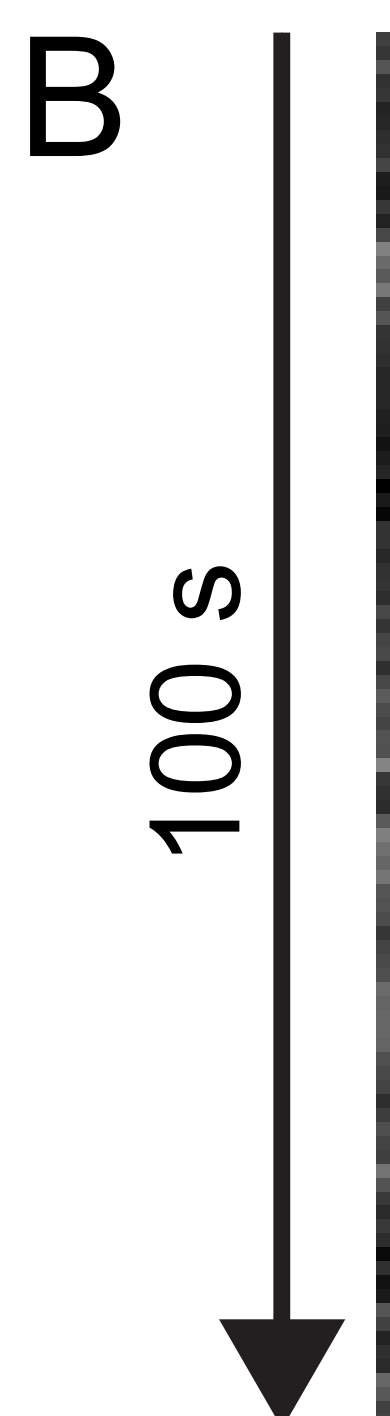

merge

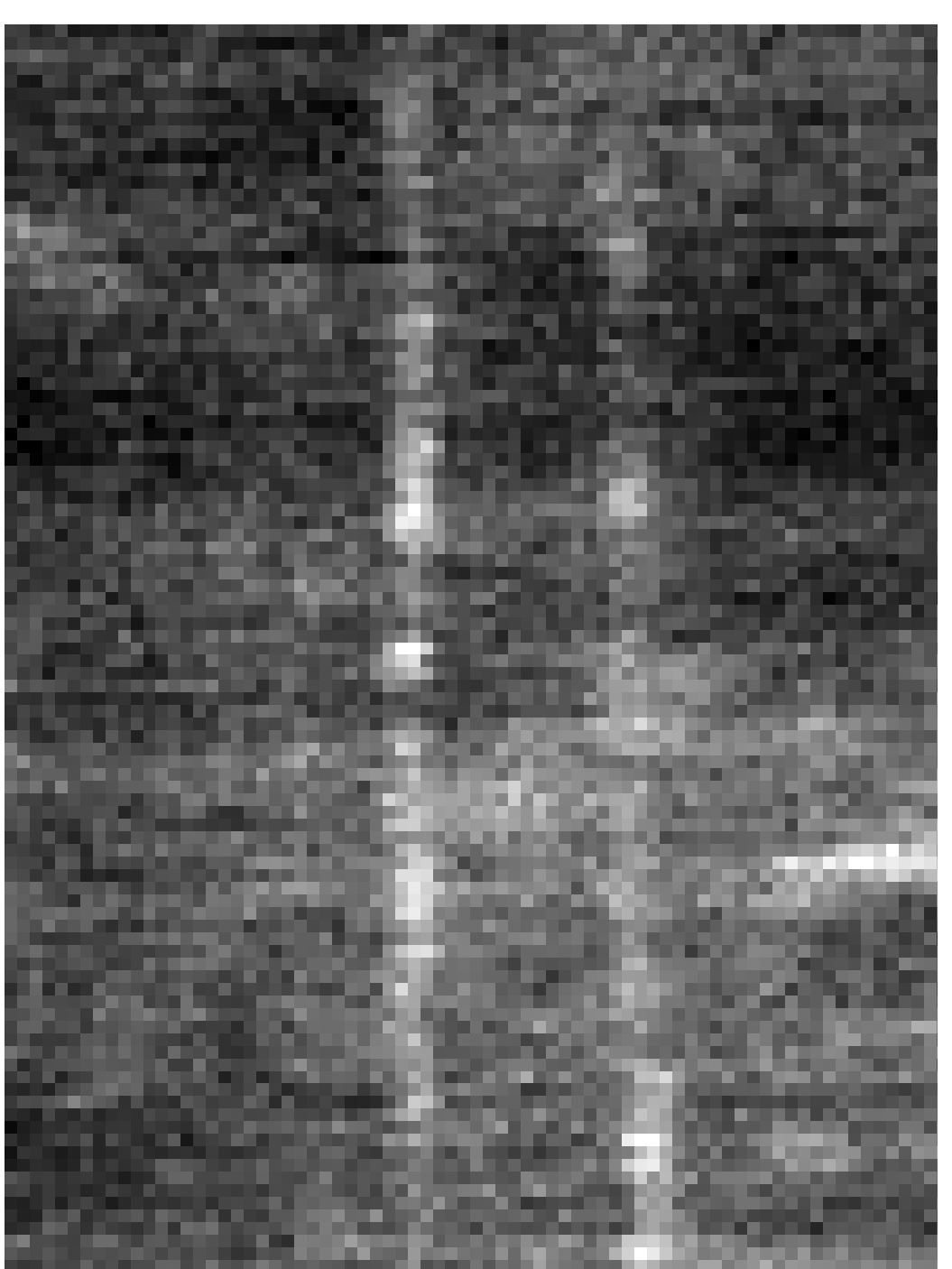

$G N_{\text {pro: }}: G N-G F P$

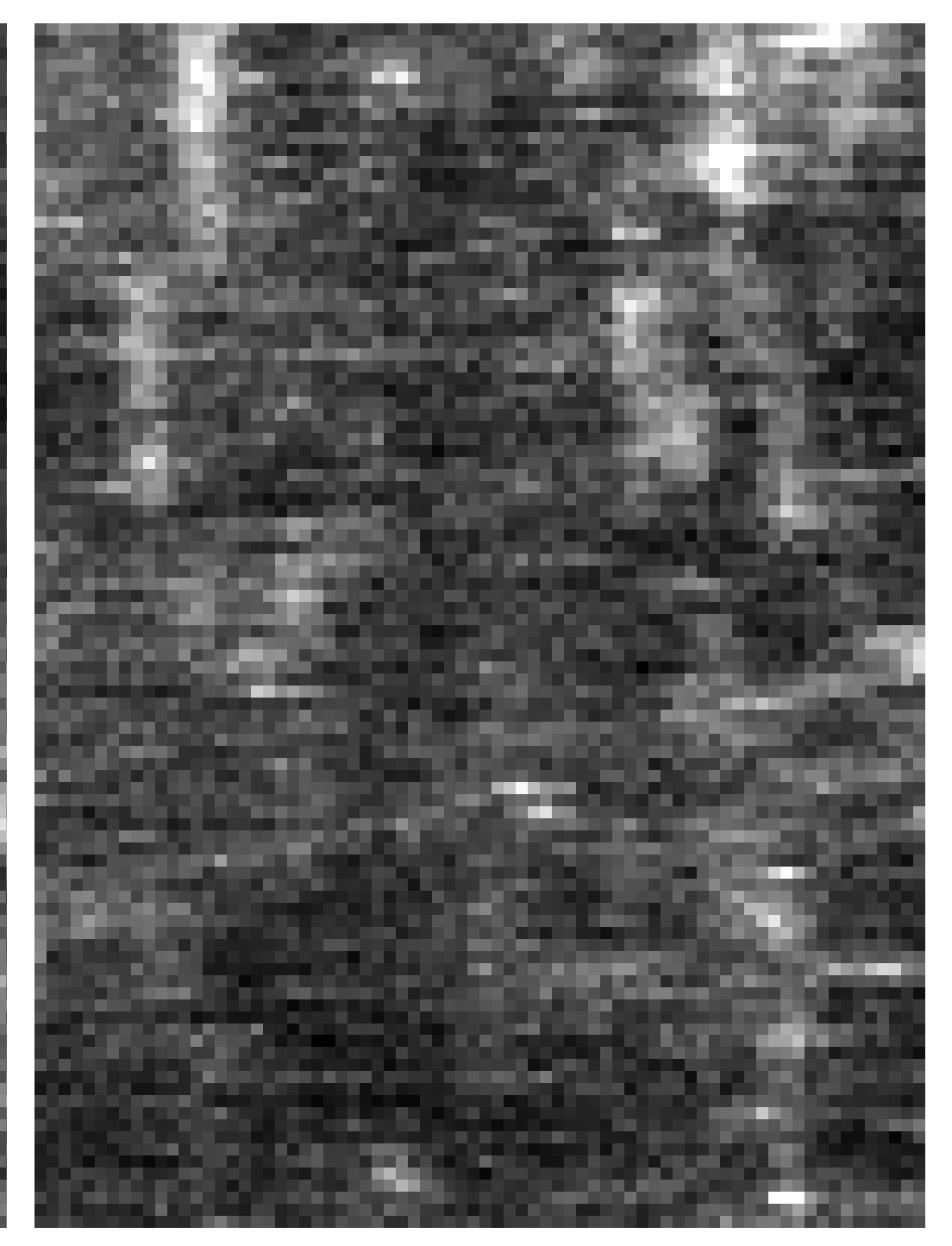

$35 S_{\text {pro }}: C L C-m K O$

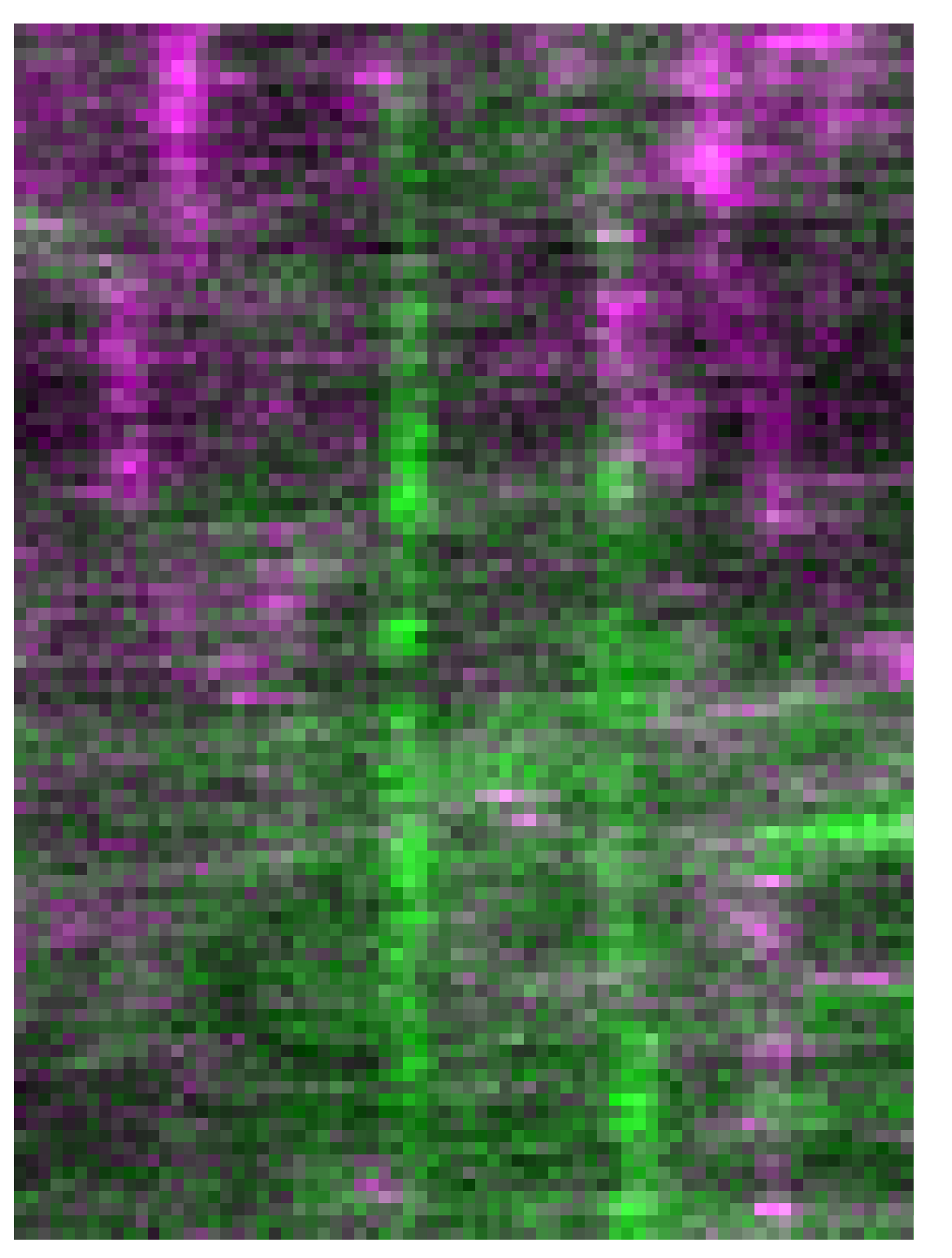

merge
D

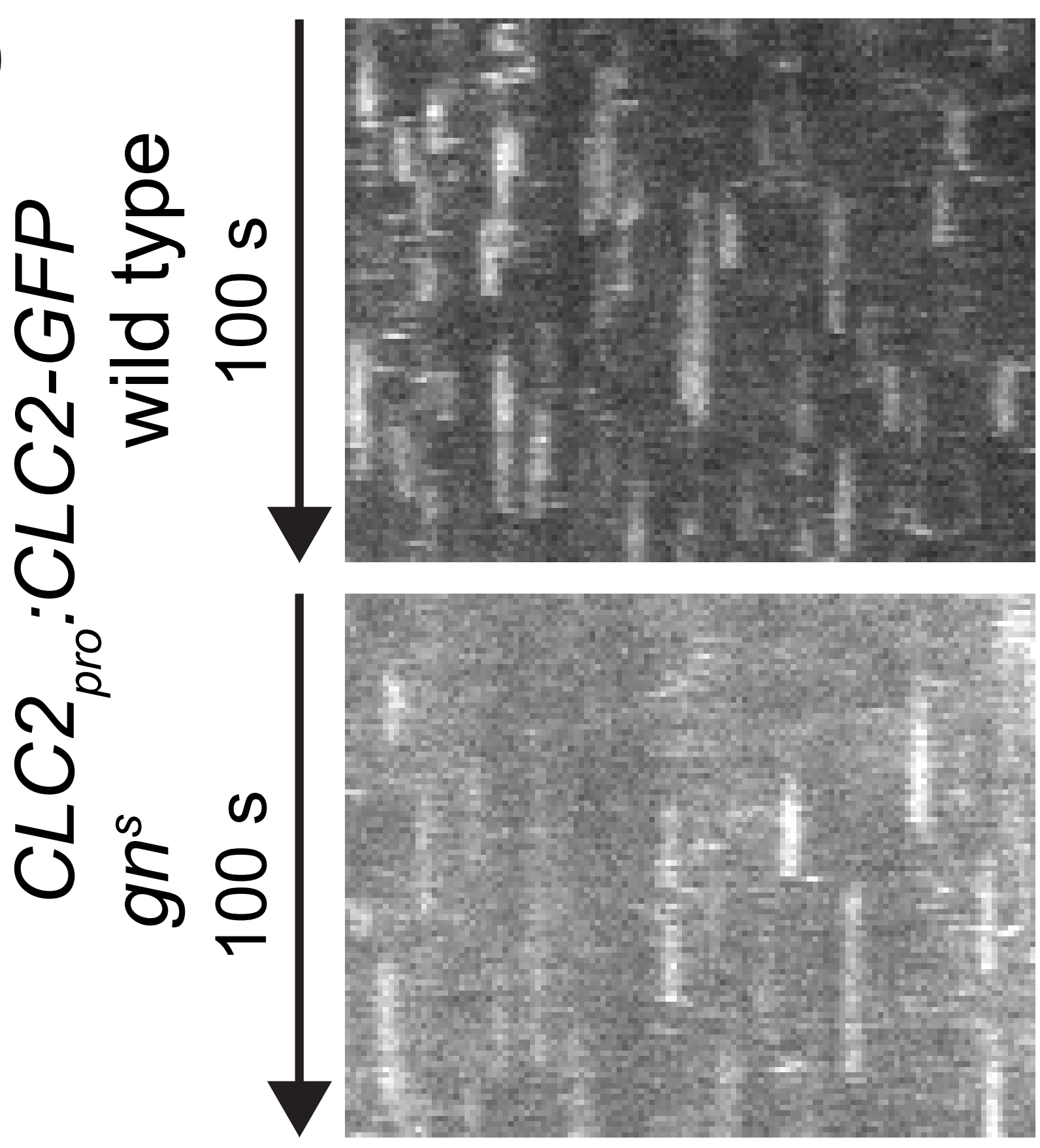

$\mathrm{F}$

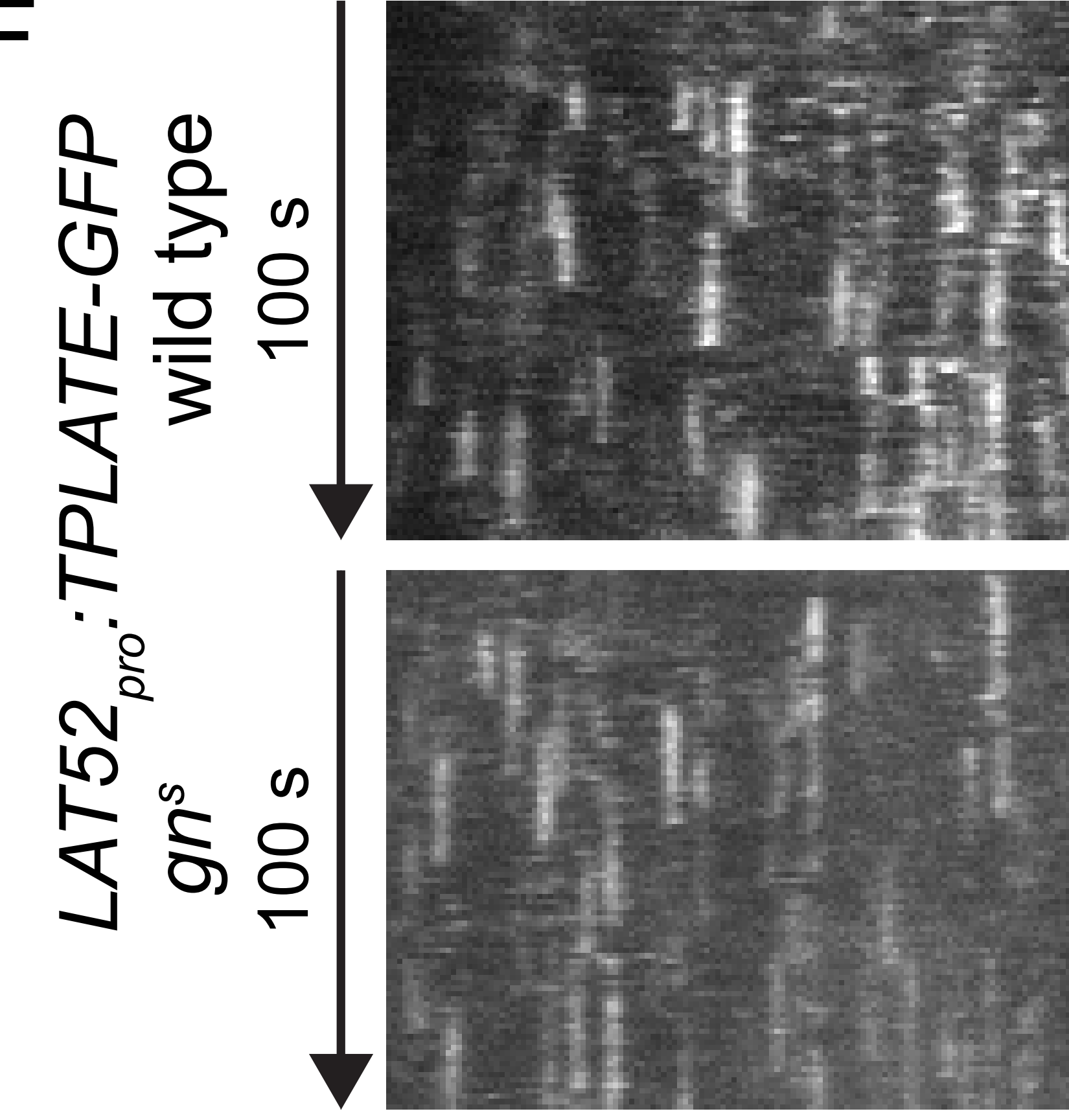

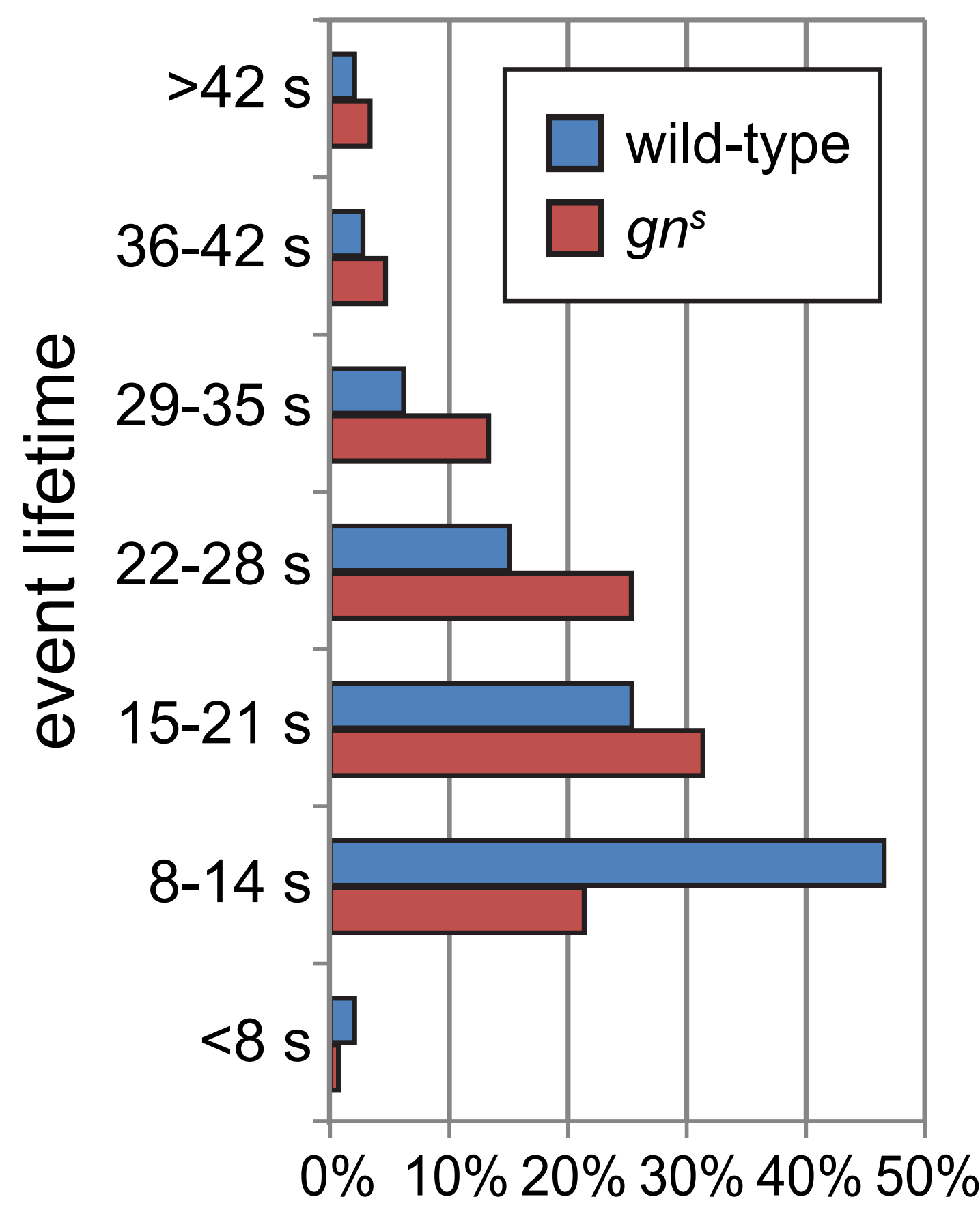

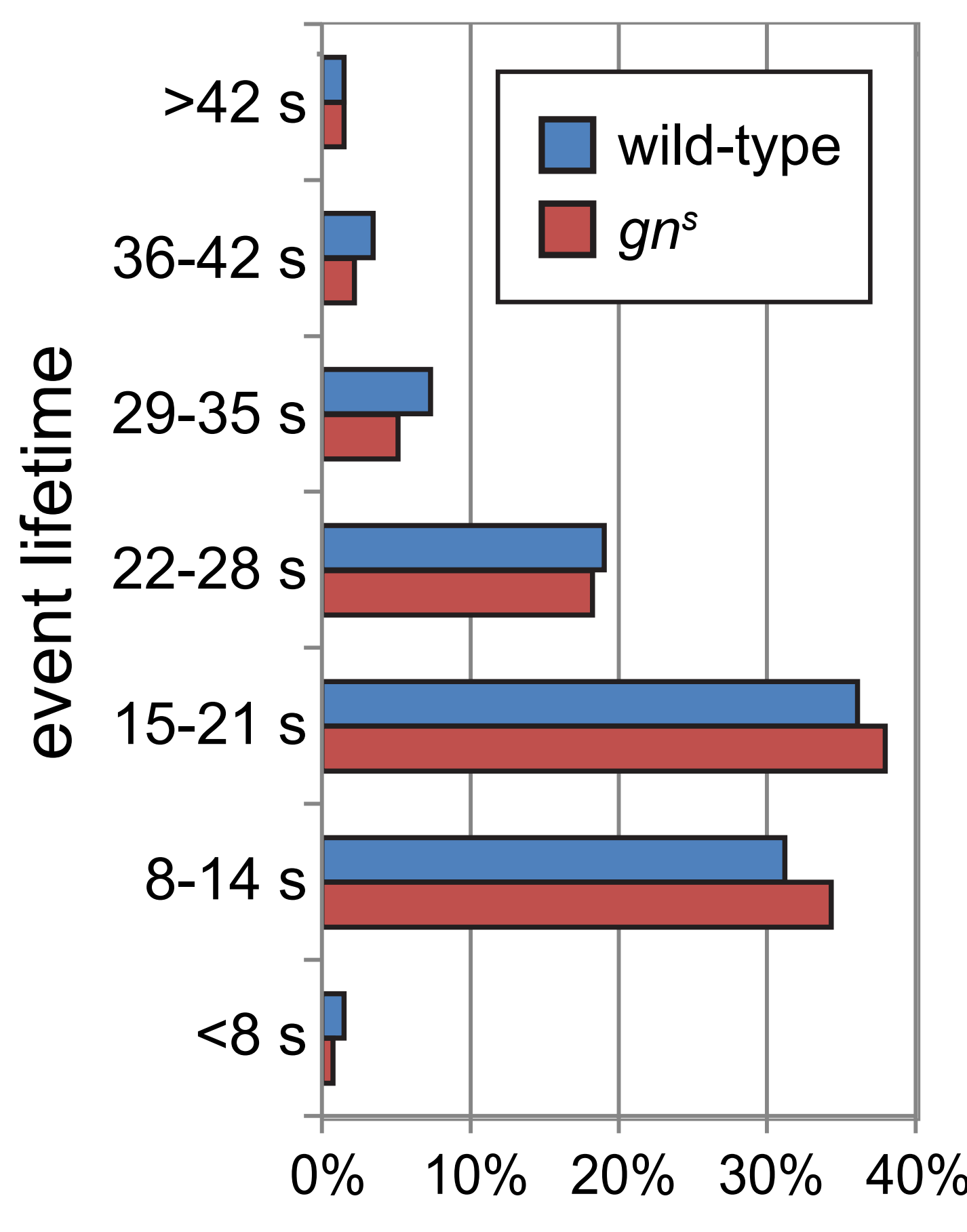


Figure 3-figure supplement 1
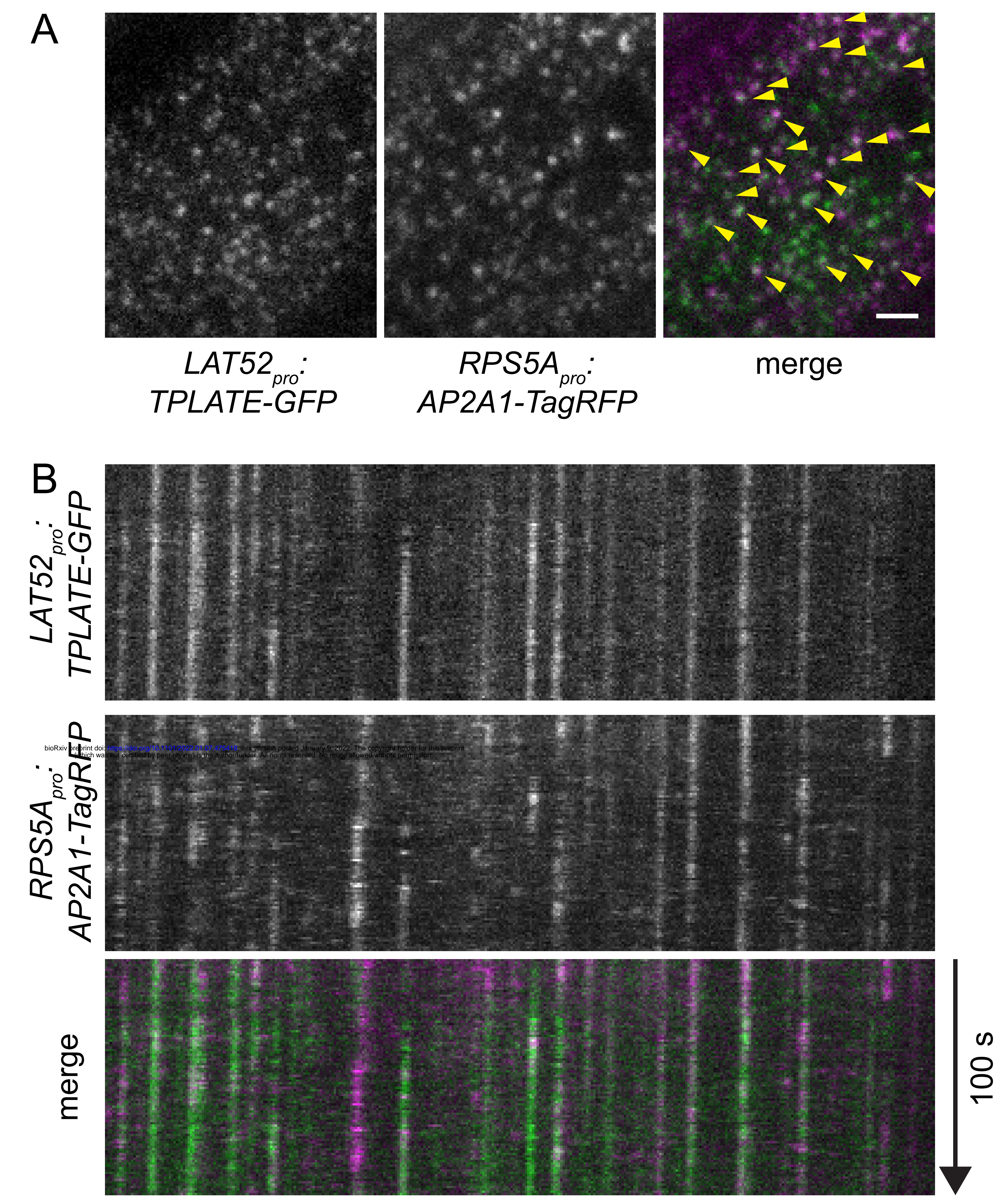
Figure 4
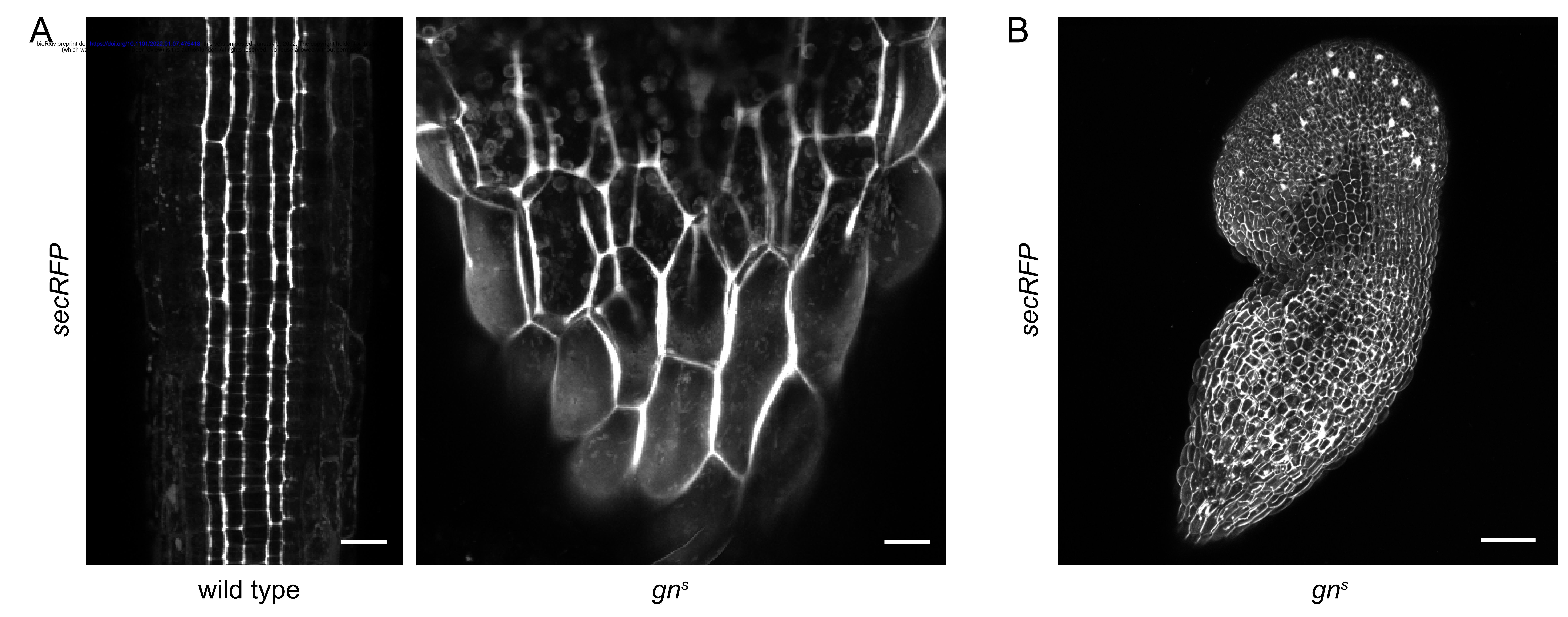
Figure 4-figure supplement 1

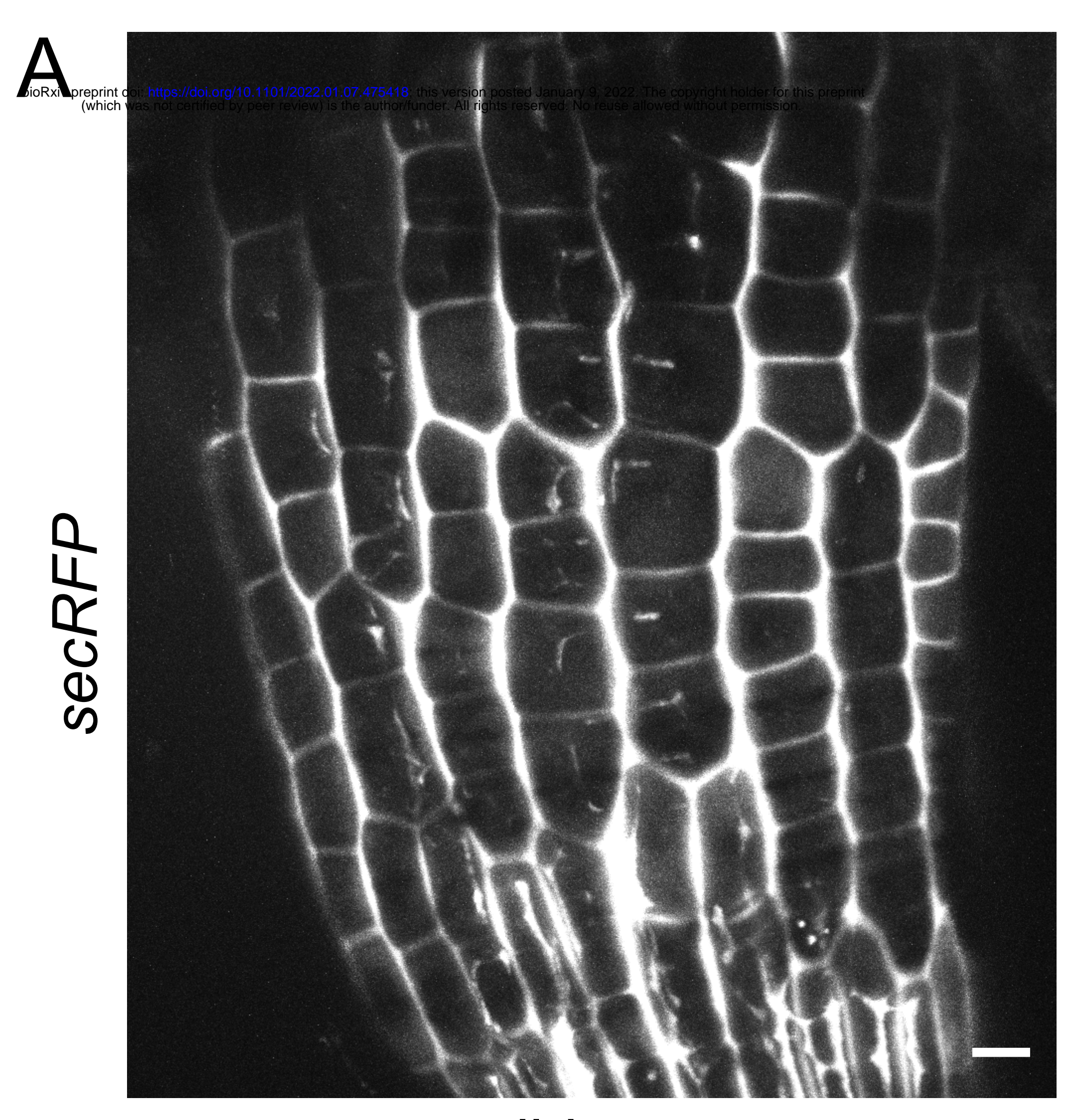

wild type

2 days old

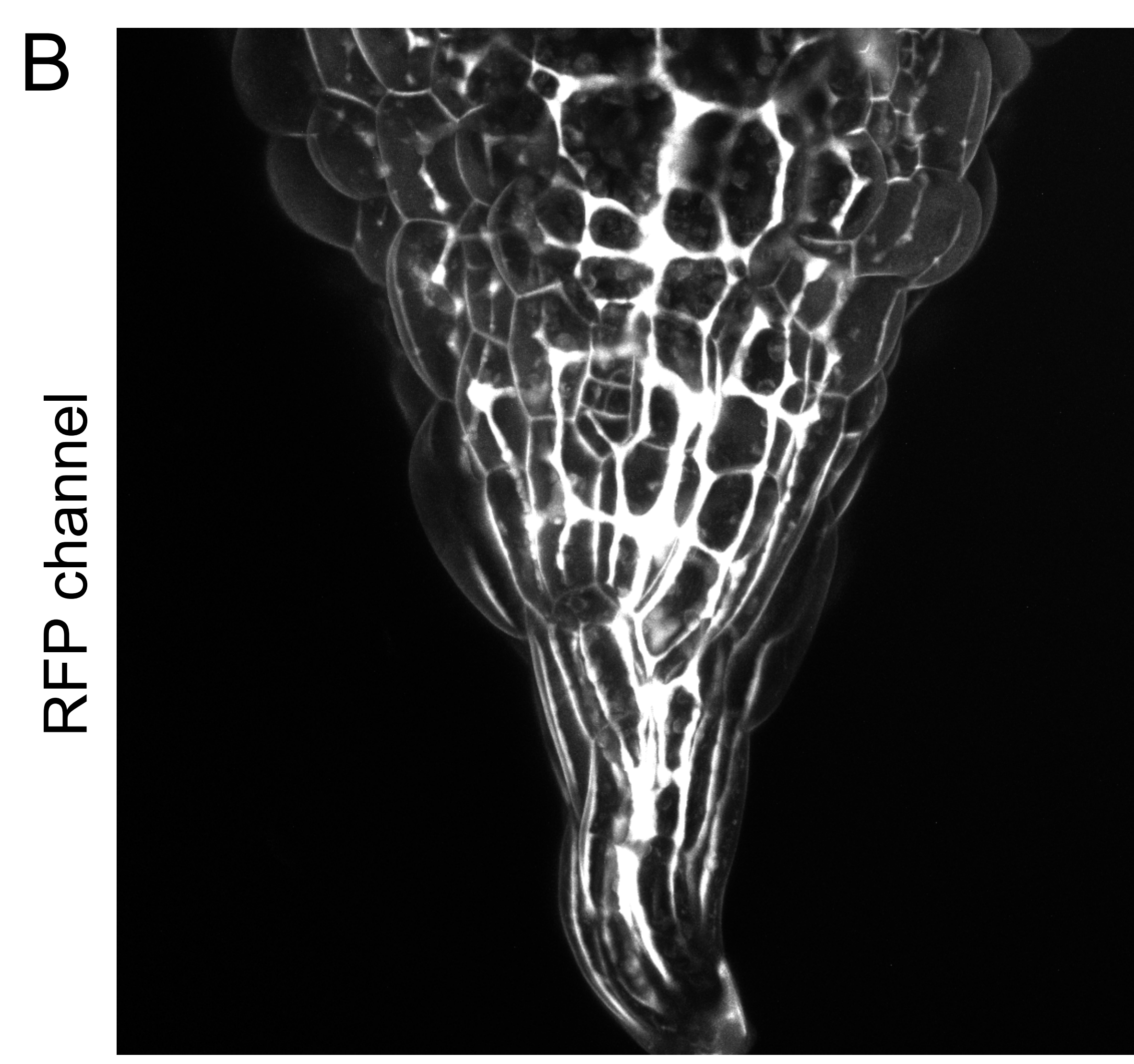

$g n^{s} \sec R F P$

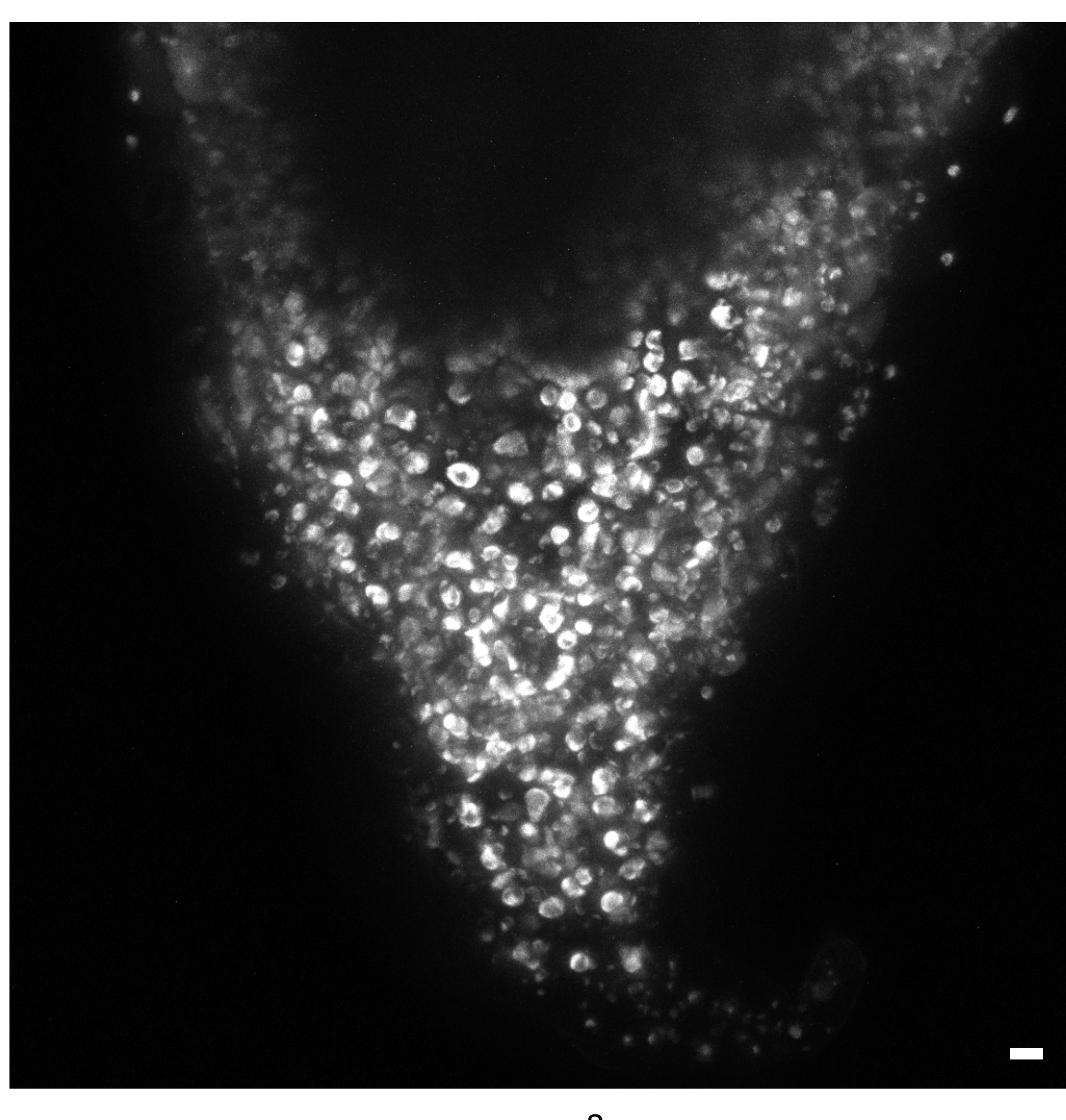

$g n^{s}$ 


\section{Figure 5}
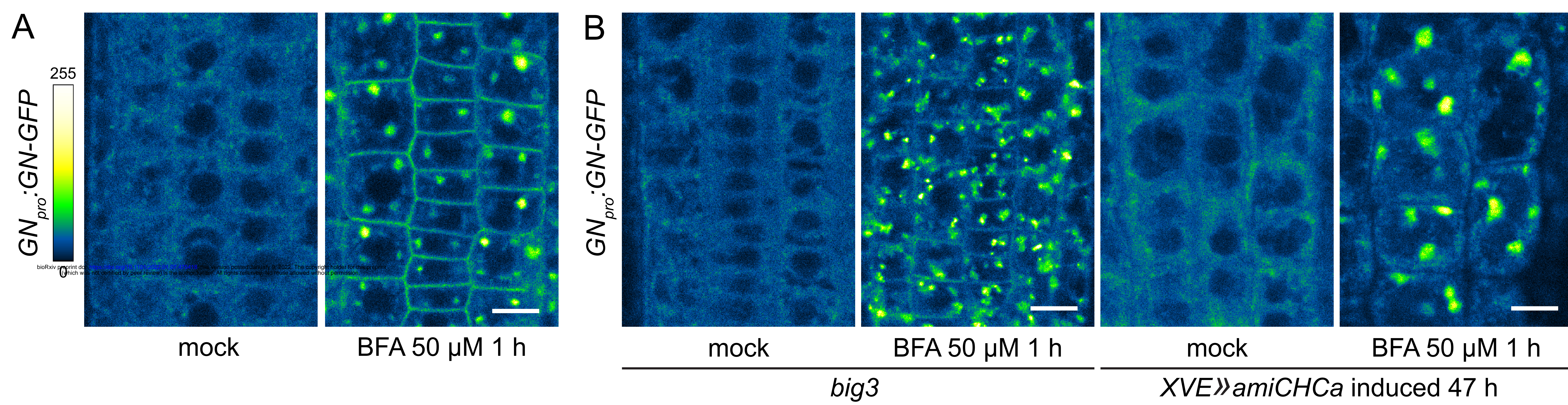
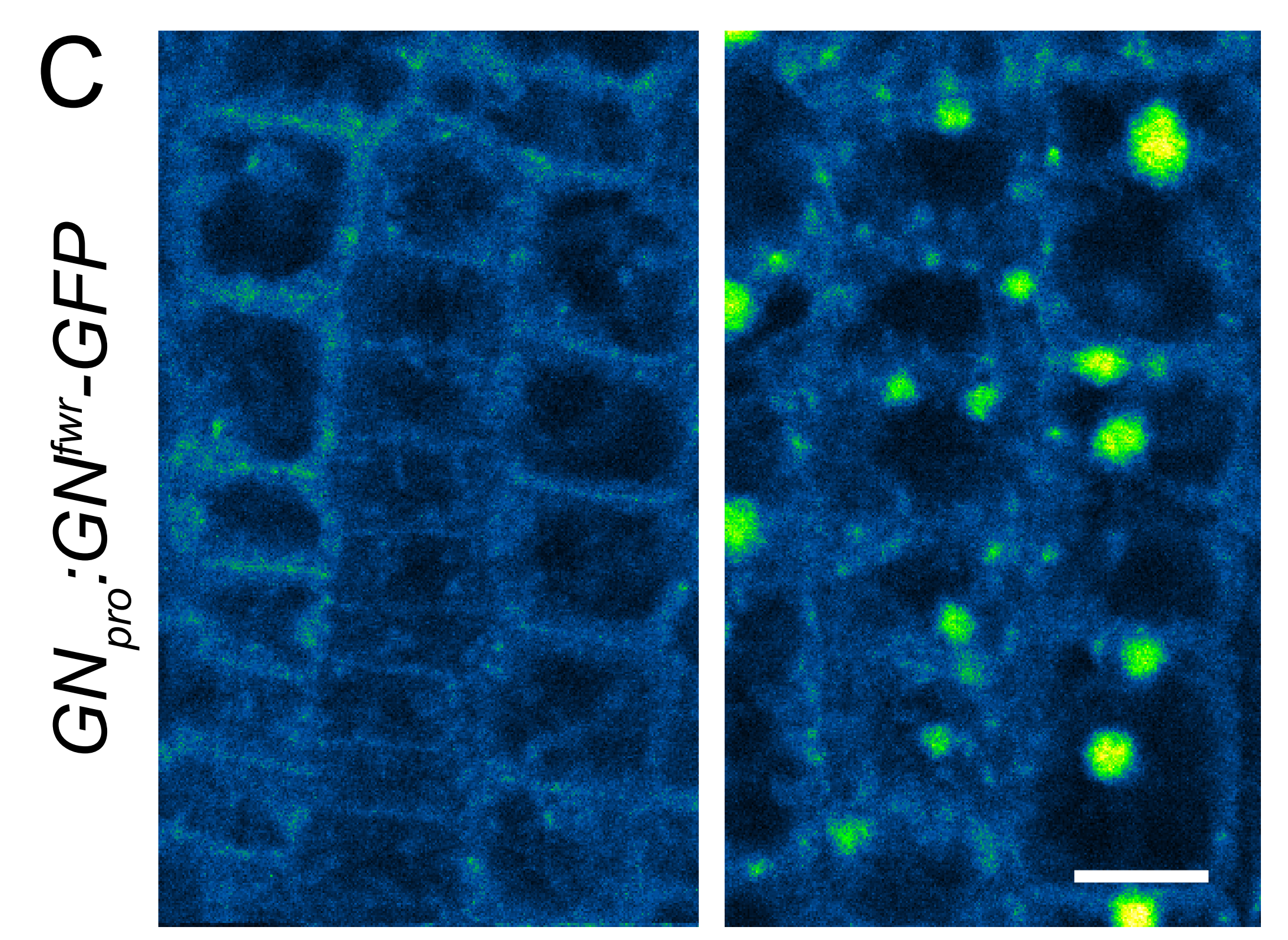

mock
BFA $50 \mu \mathrm{M} 1 \mathrm{~h}$

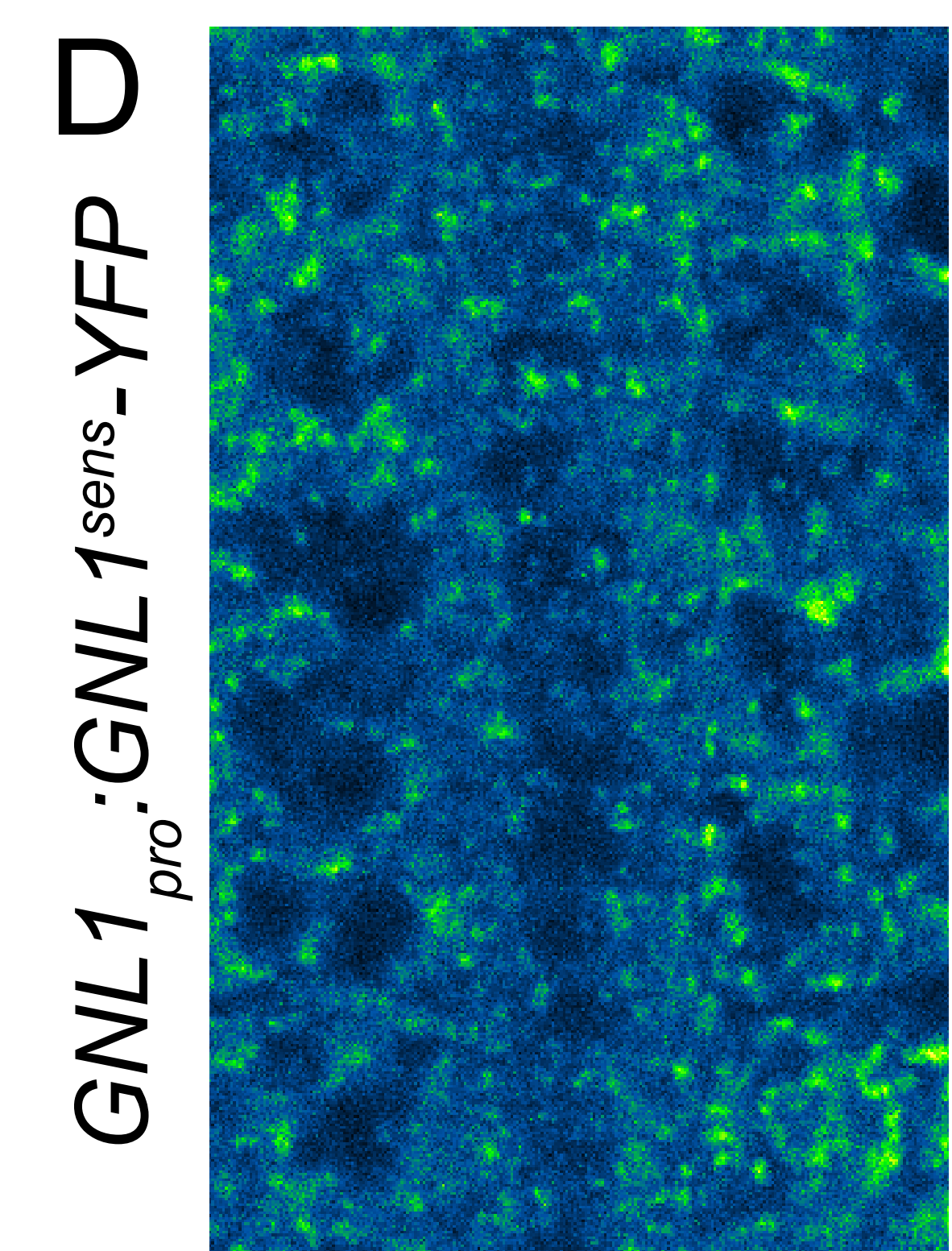

mock

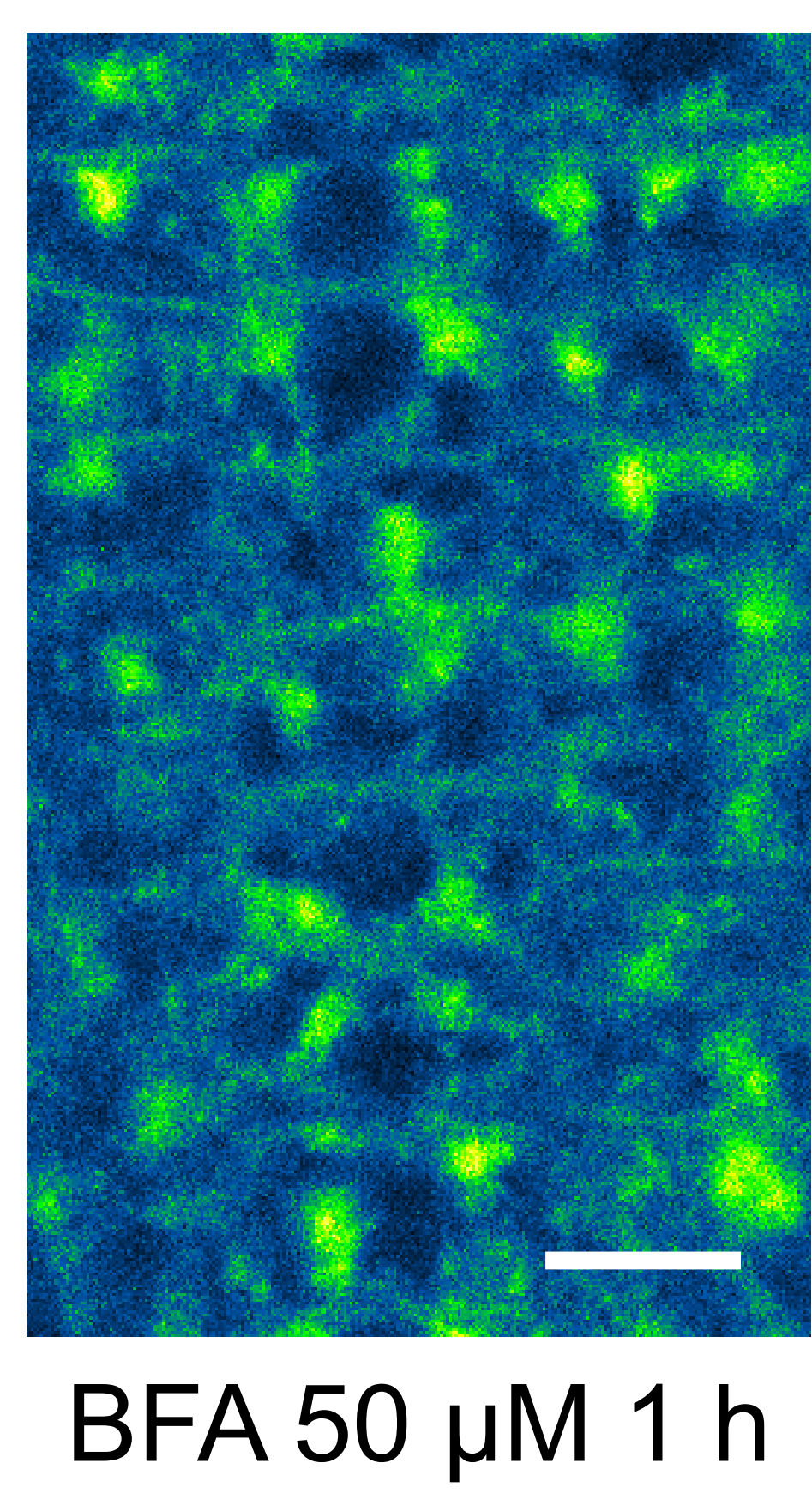

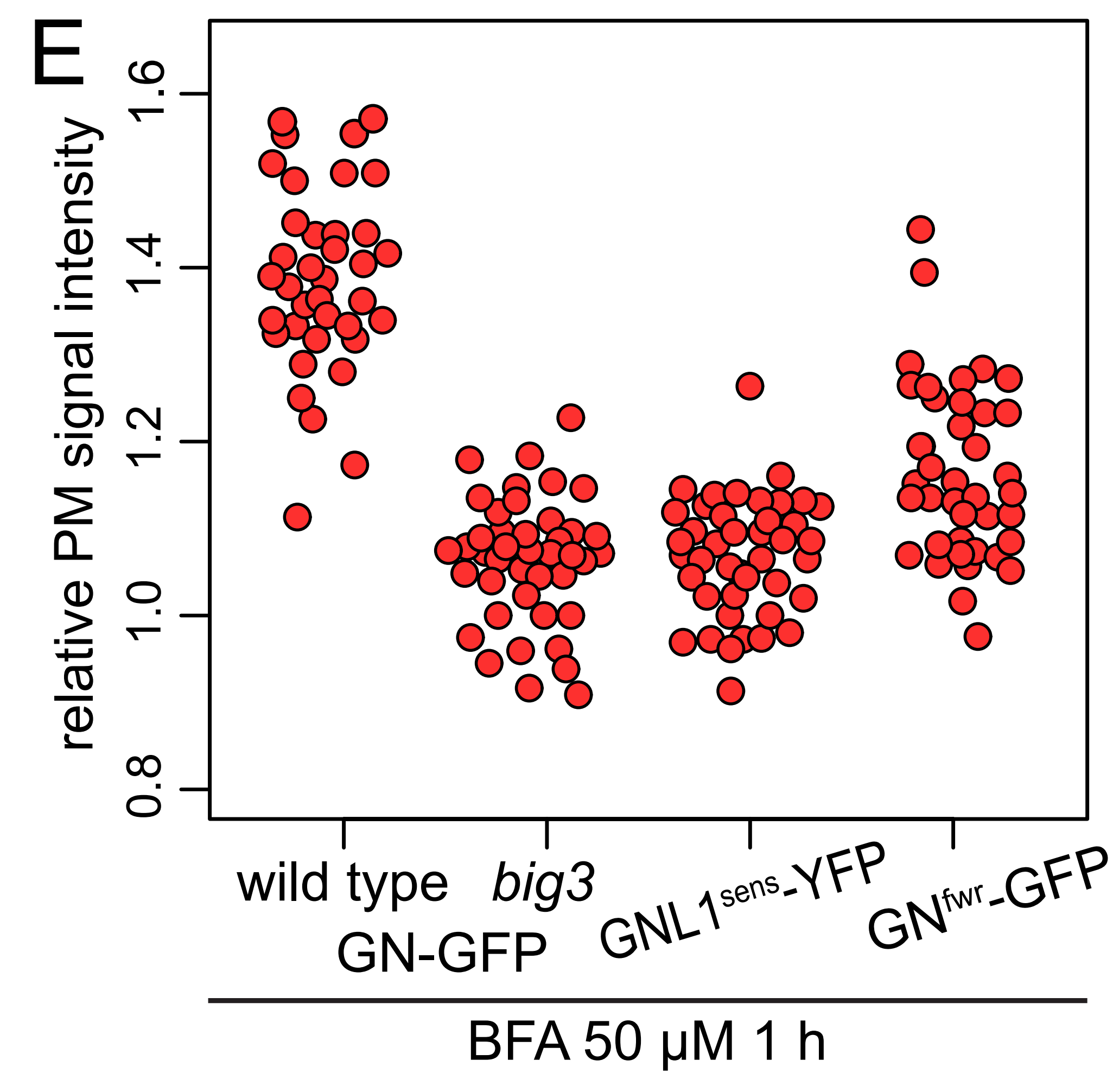

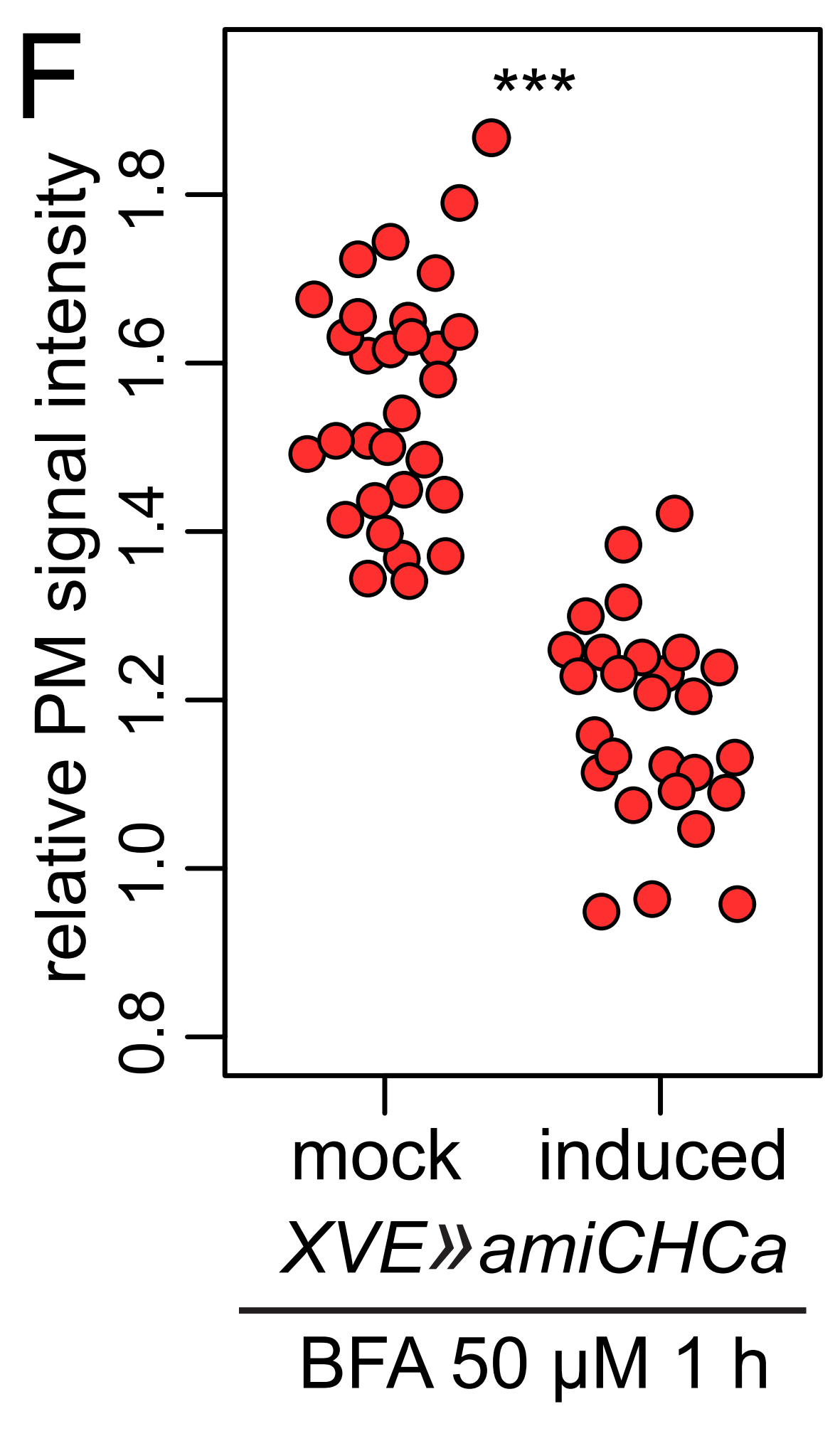


Figure 5-figure supplement 1
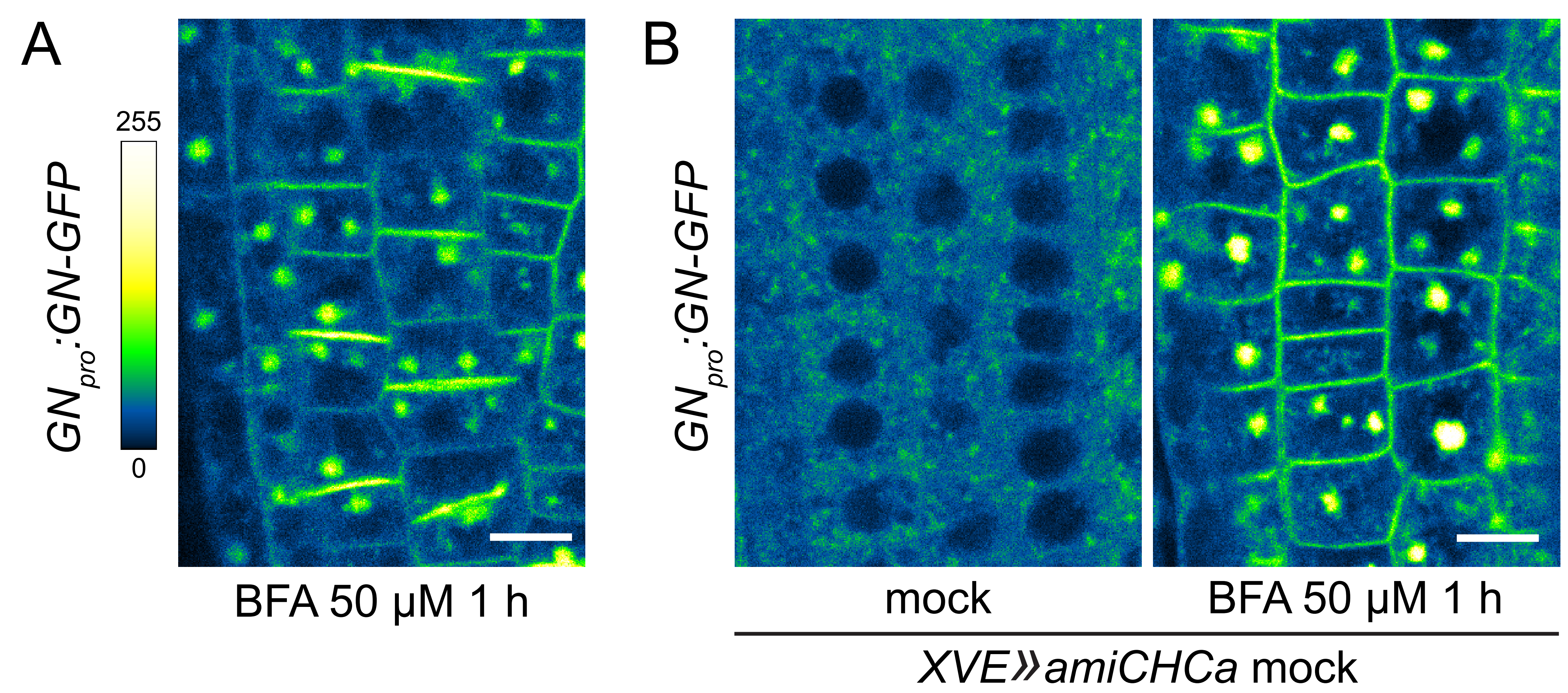

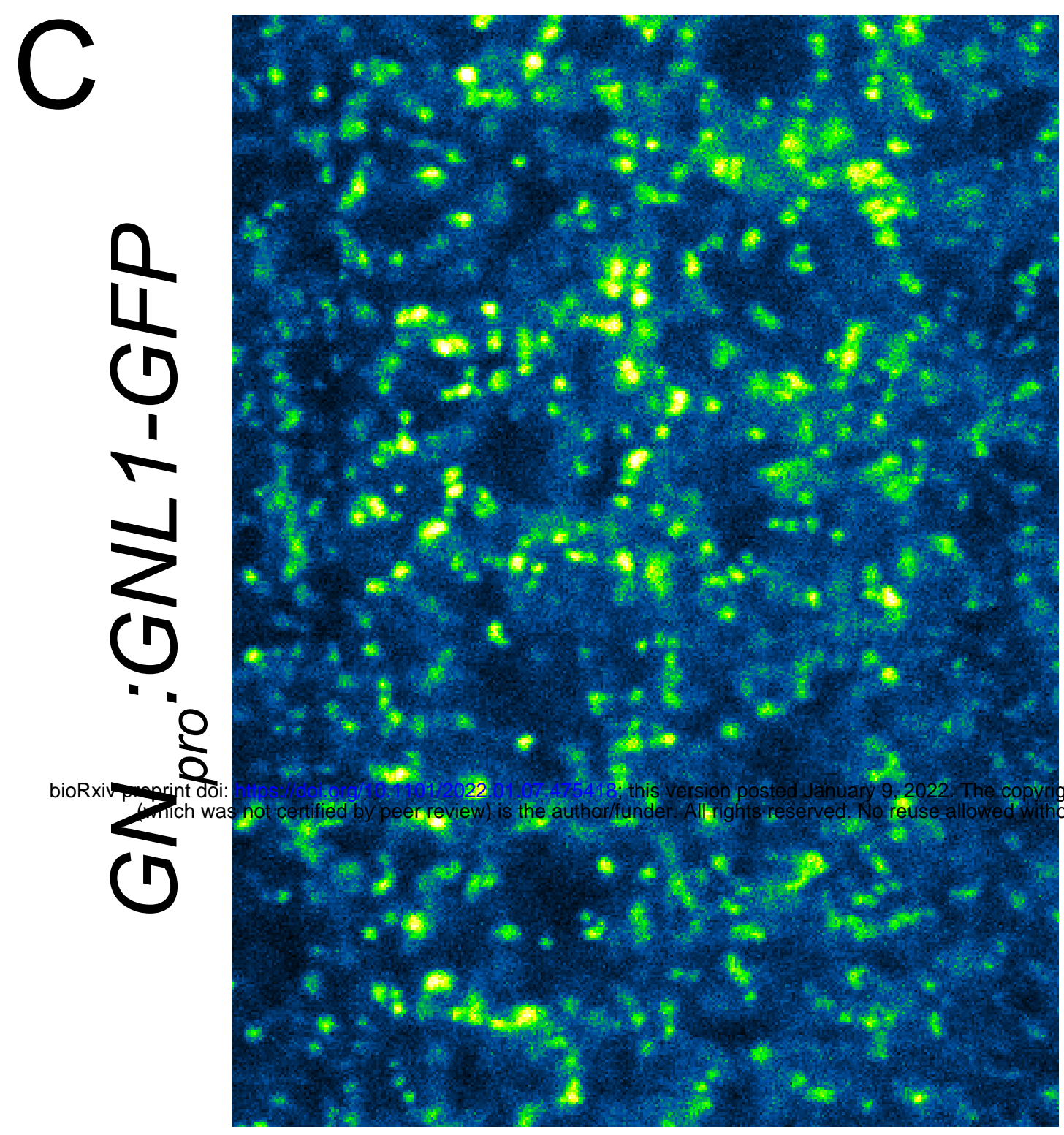

mock

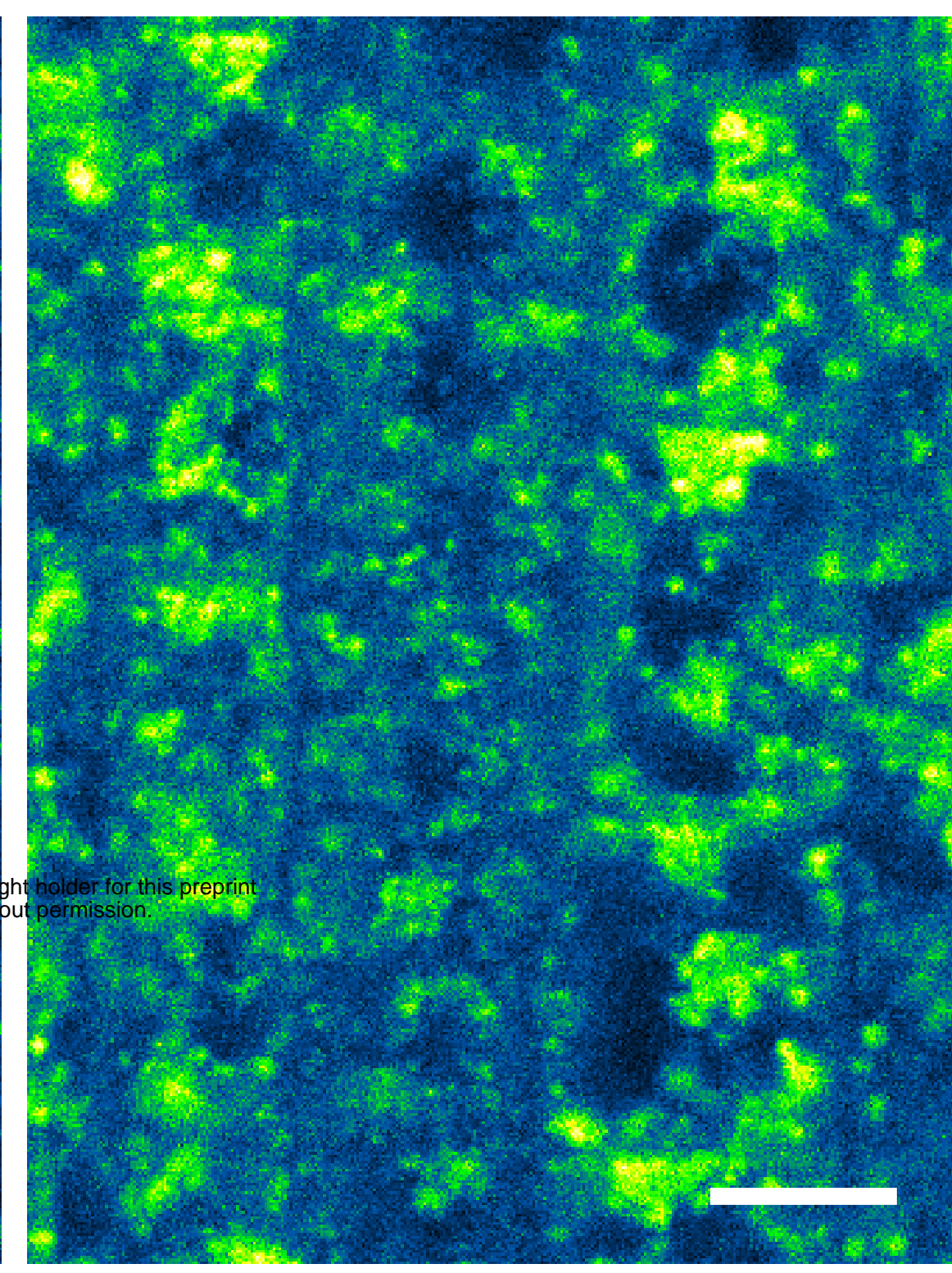

BFA $50 \mu \mathrm{M} 1 \mathrm{~h}$

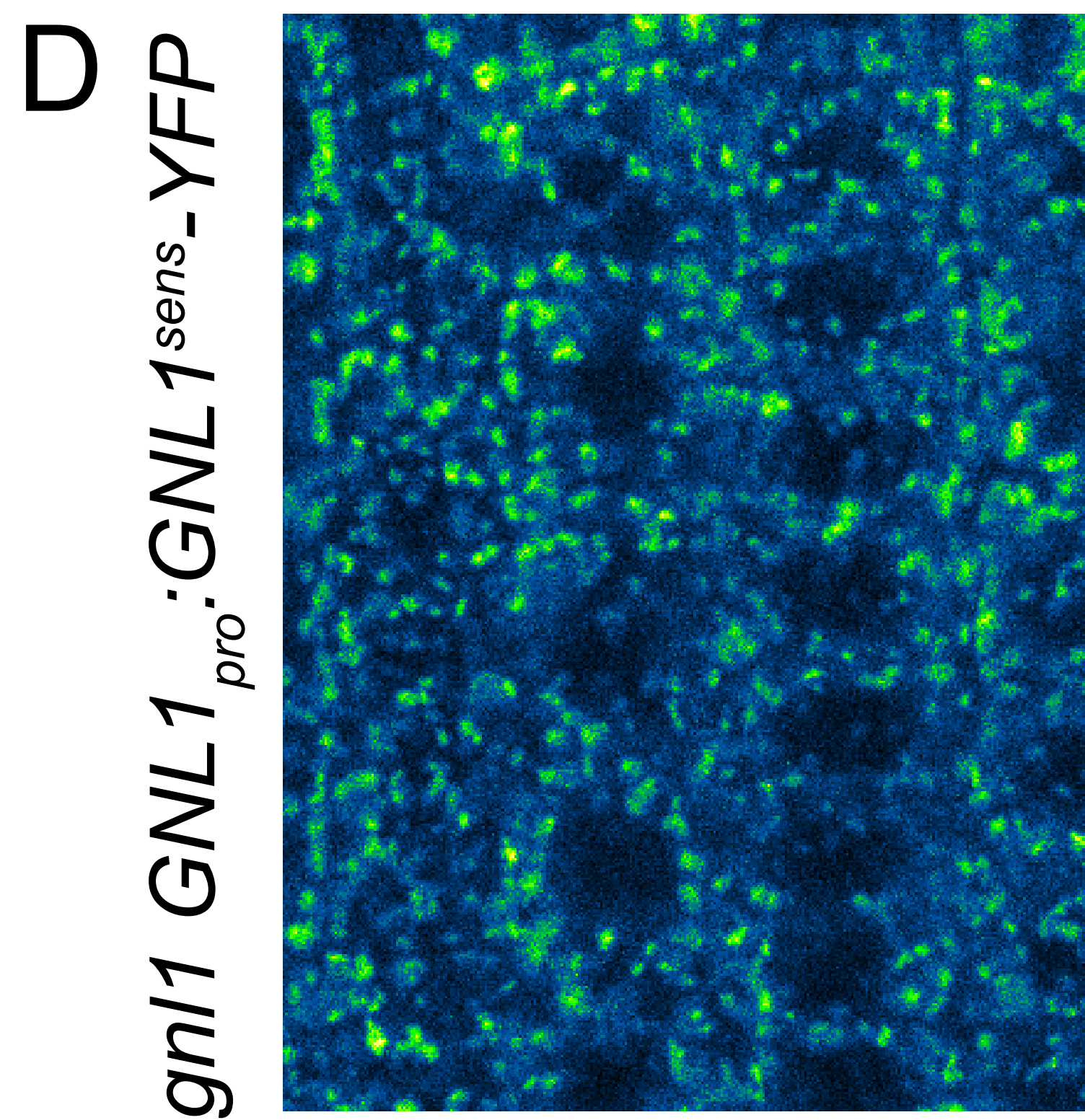

mock

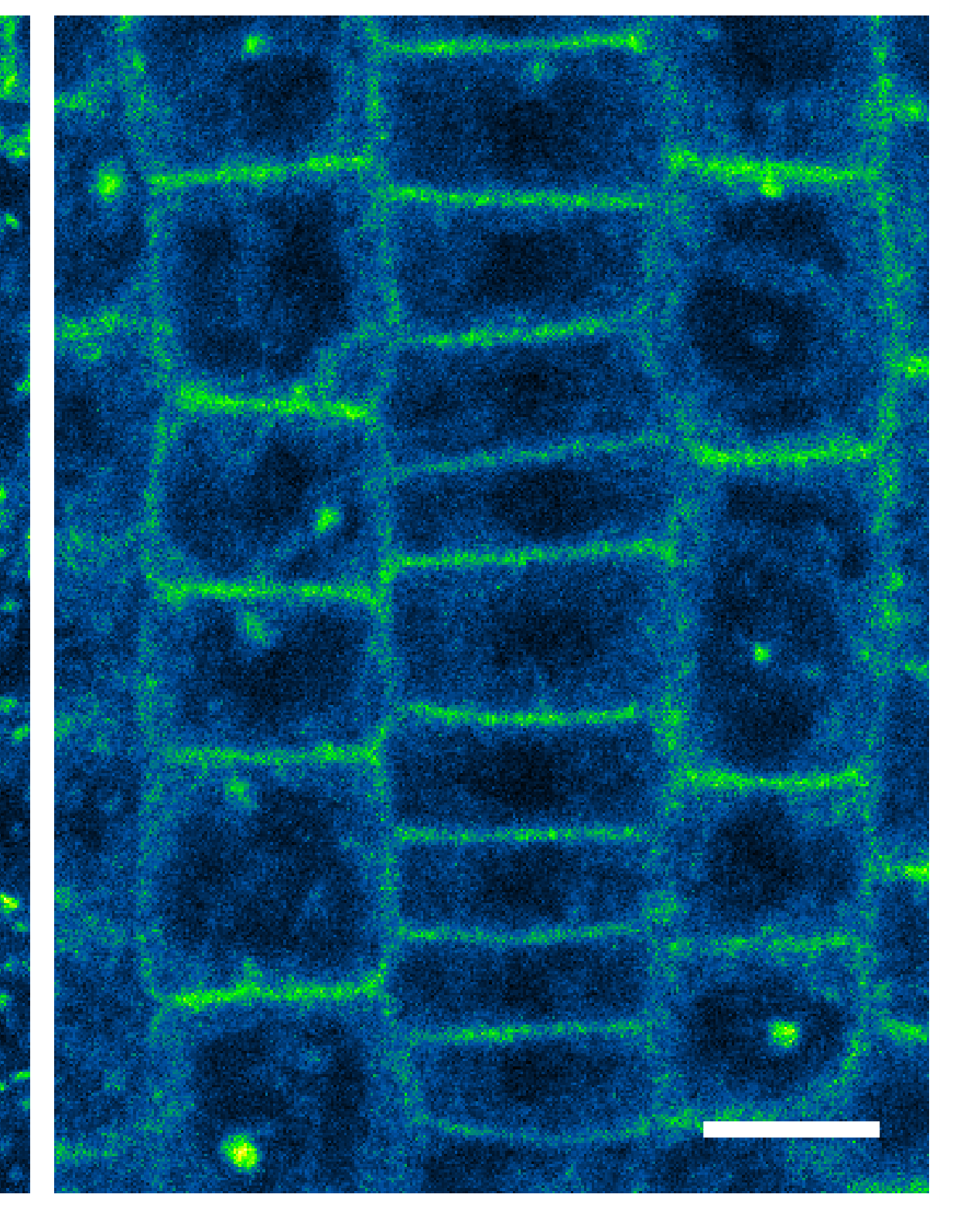

BFA $50 \mu \mathrm{M} 1 \mathrm{~h}$

E

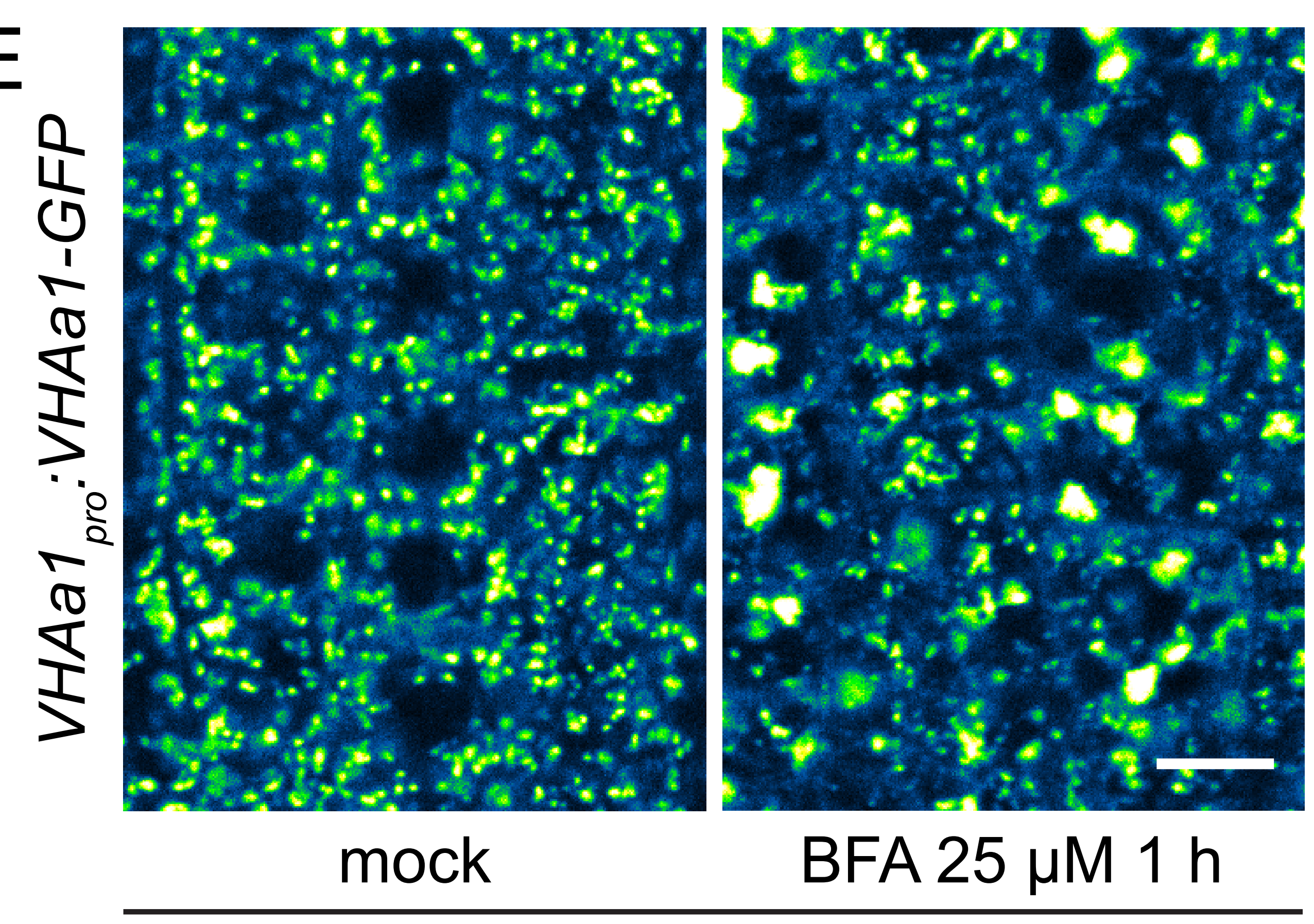

wild type

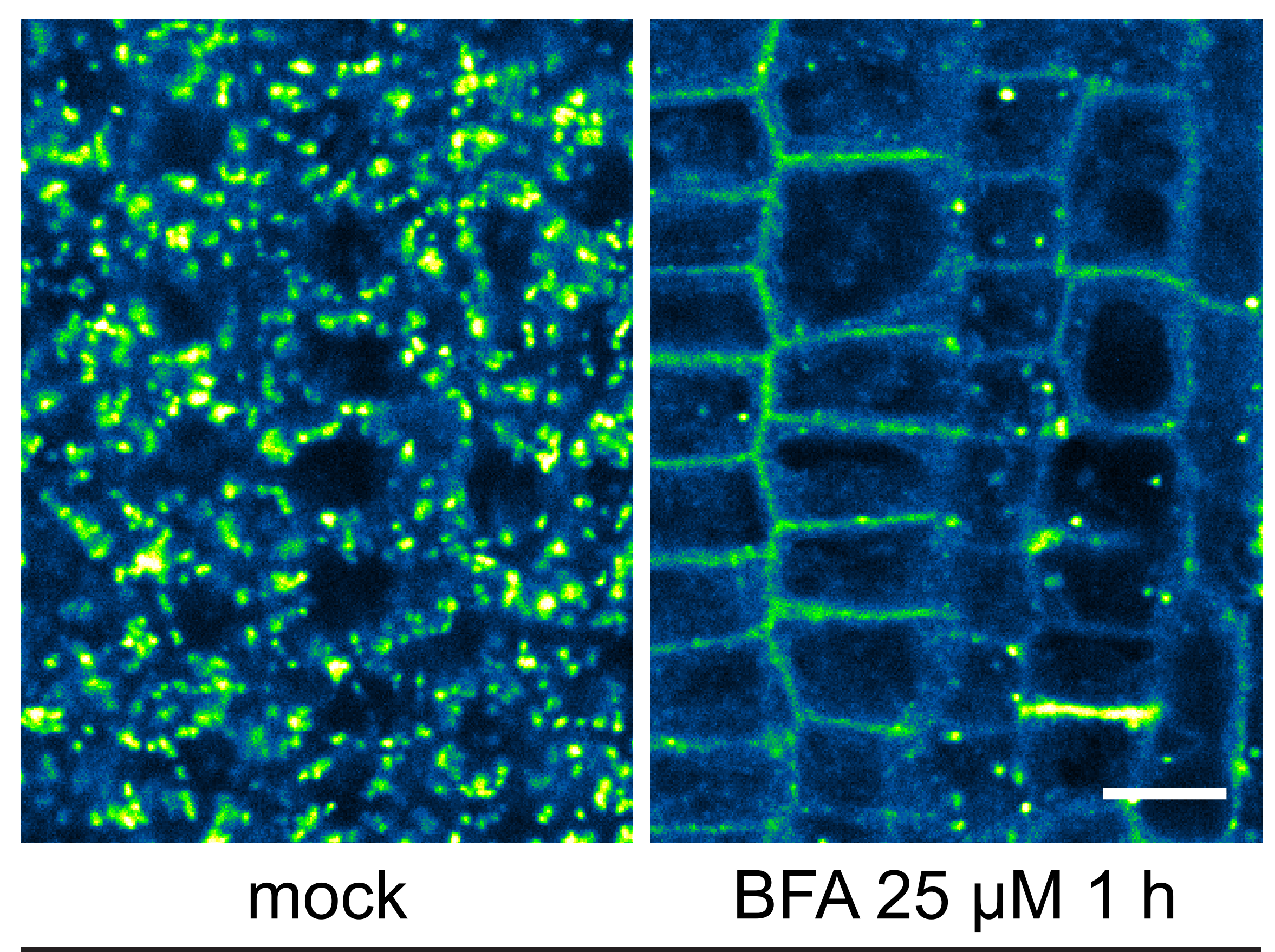

gnl1-2 
Figure 6

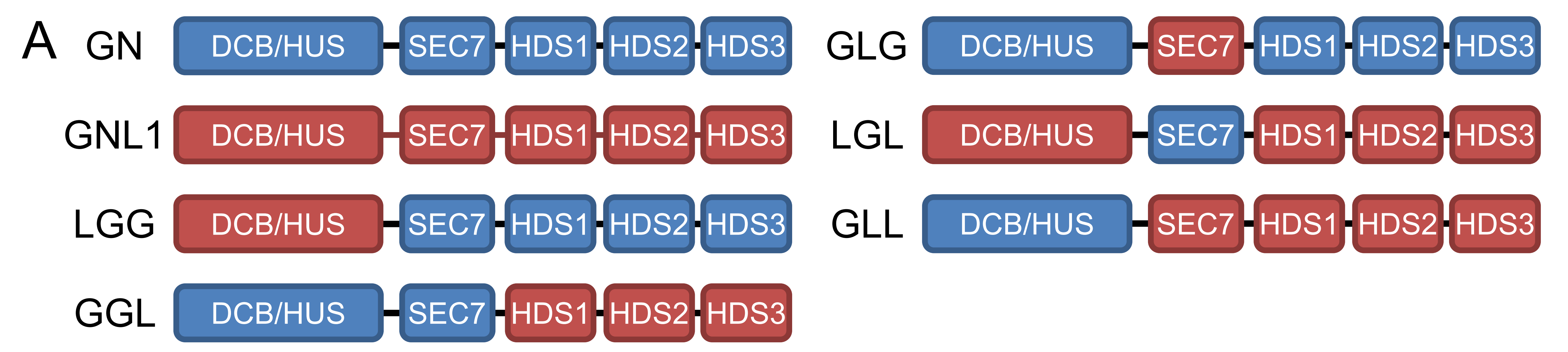

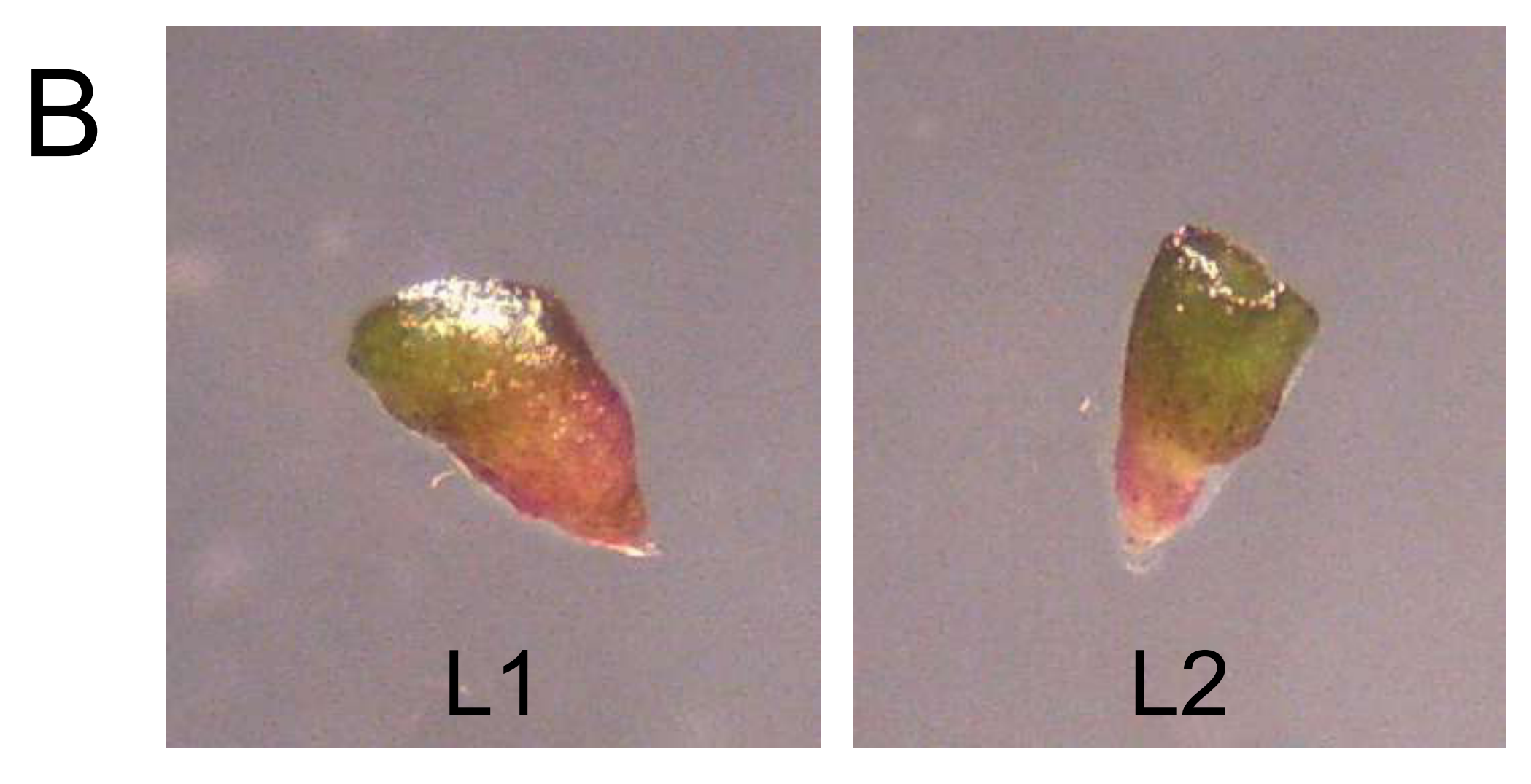

$g n^{s} G N_{\text {pro }}: G L L-G F P$

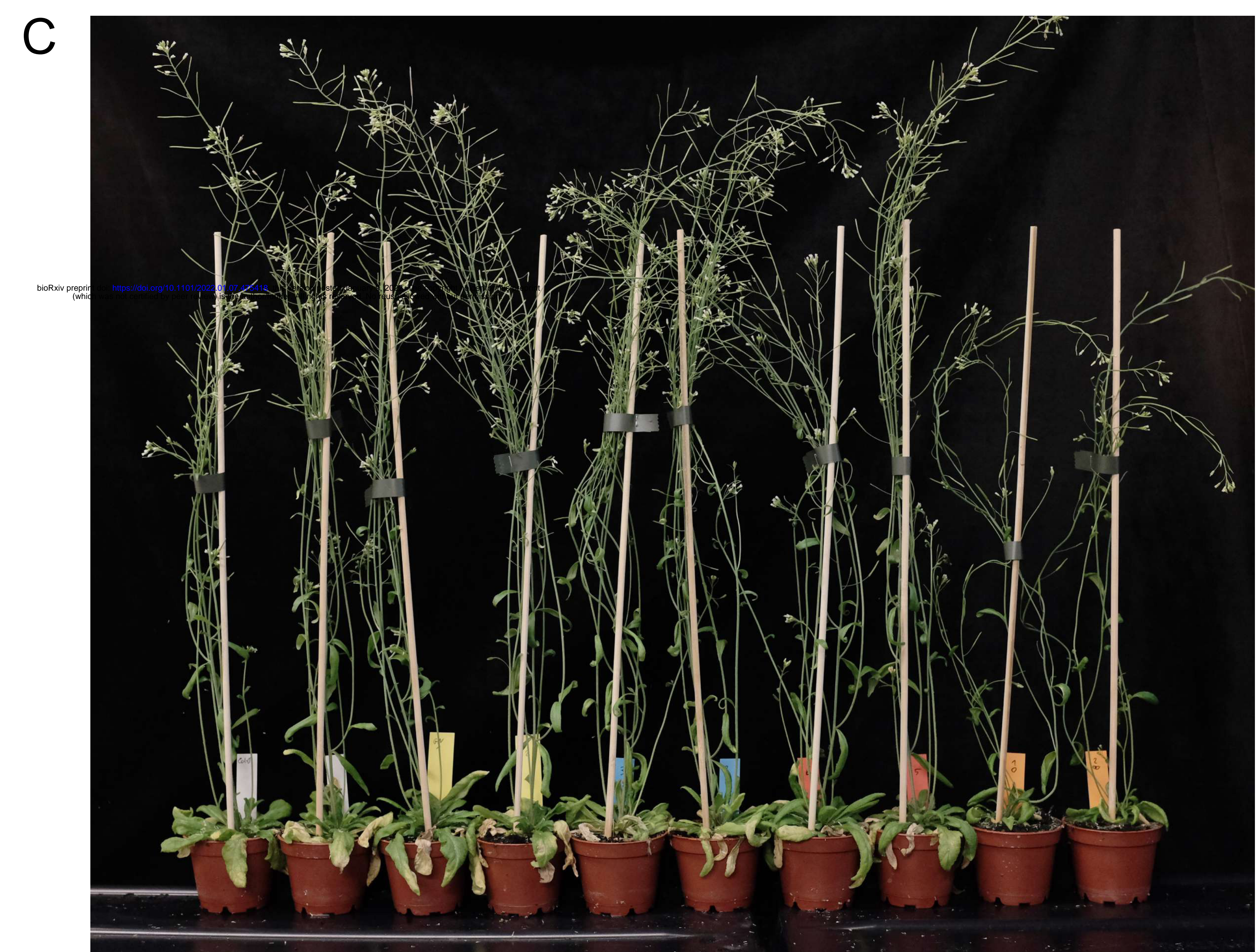

D

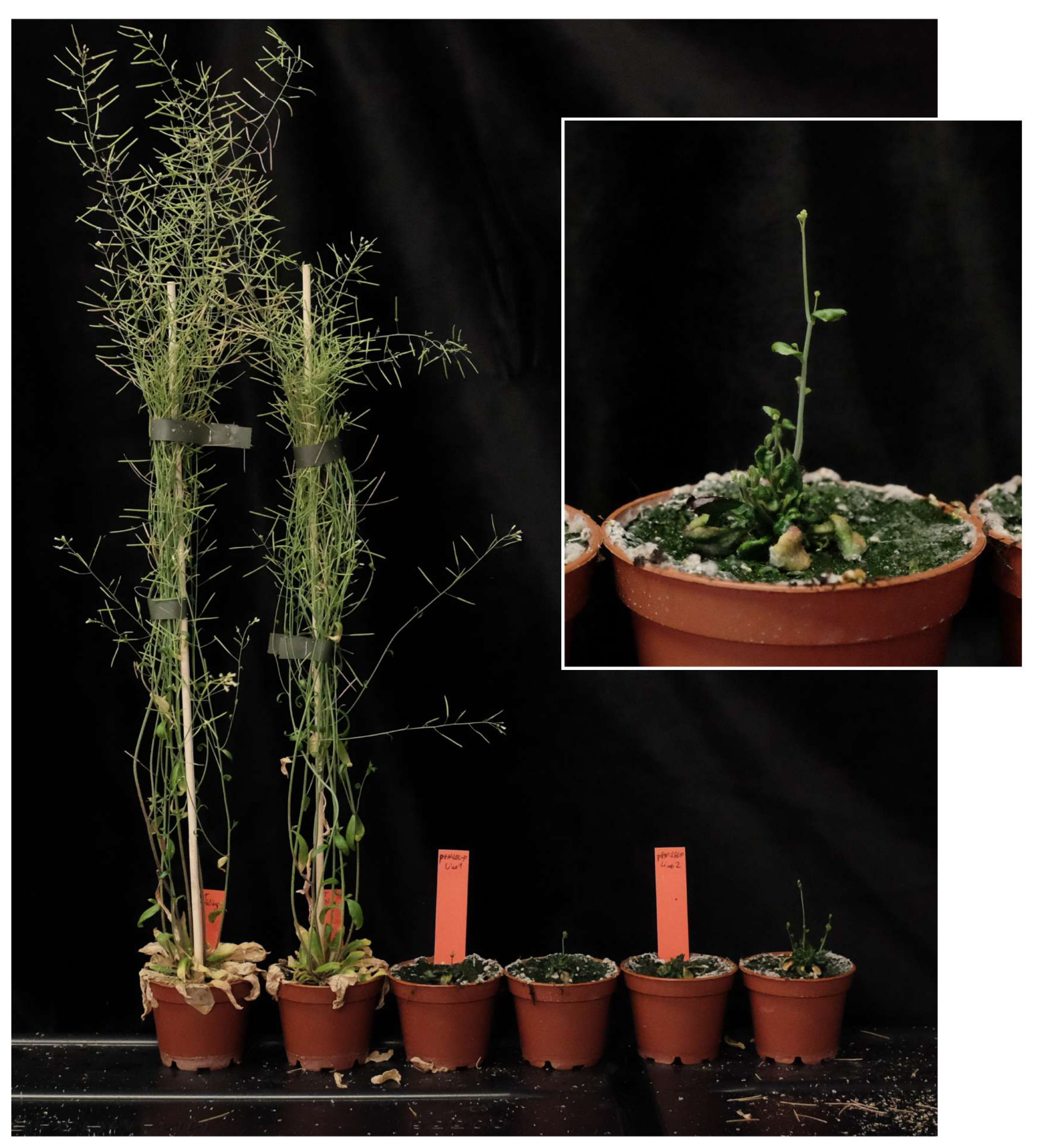

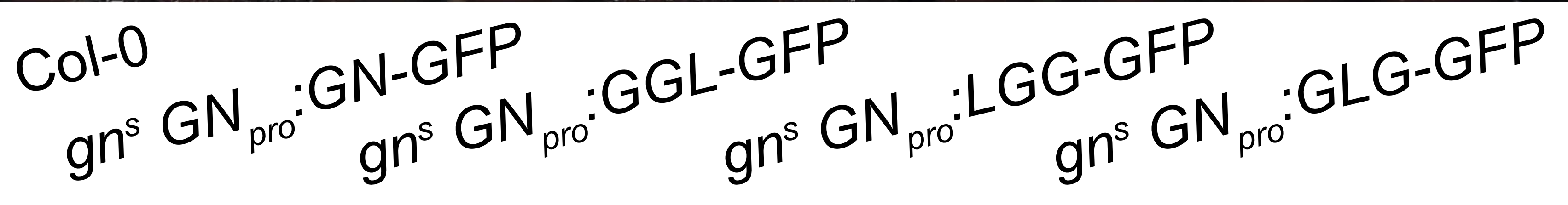


Figure 6-figure supplement 1

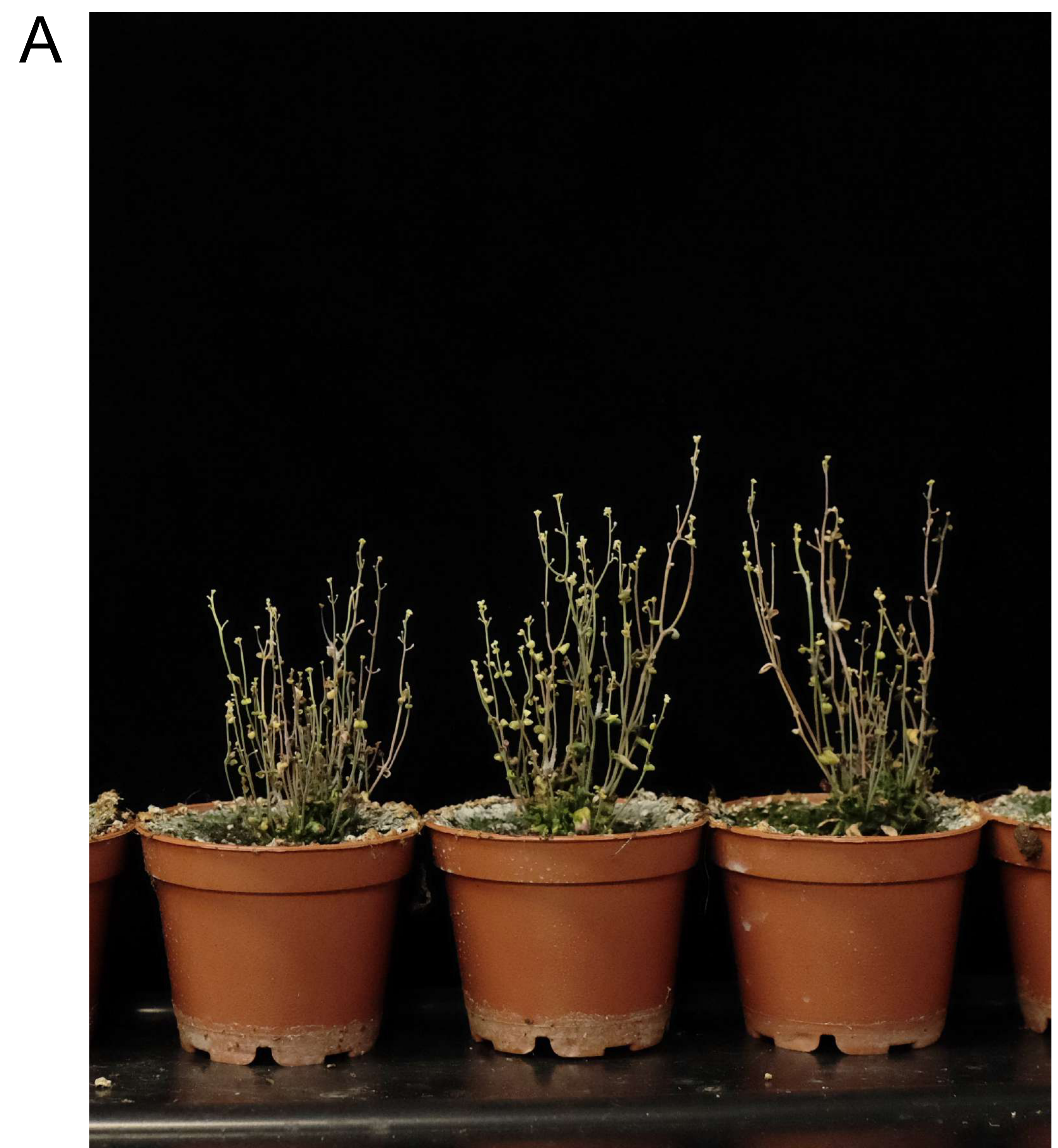

$g n^{s} G N_{\text {pro }}: L G L-G F P$
B

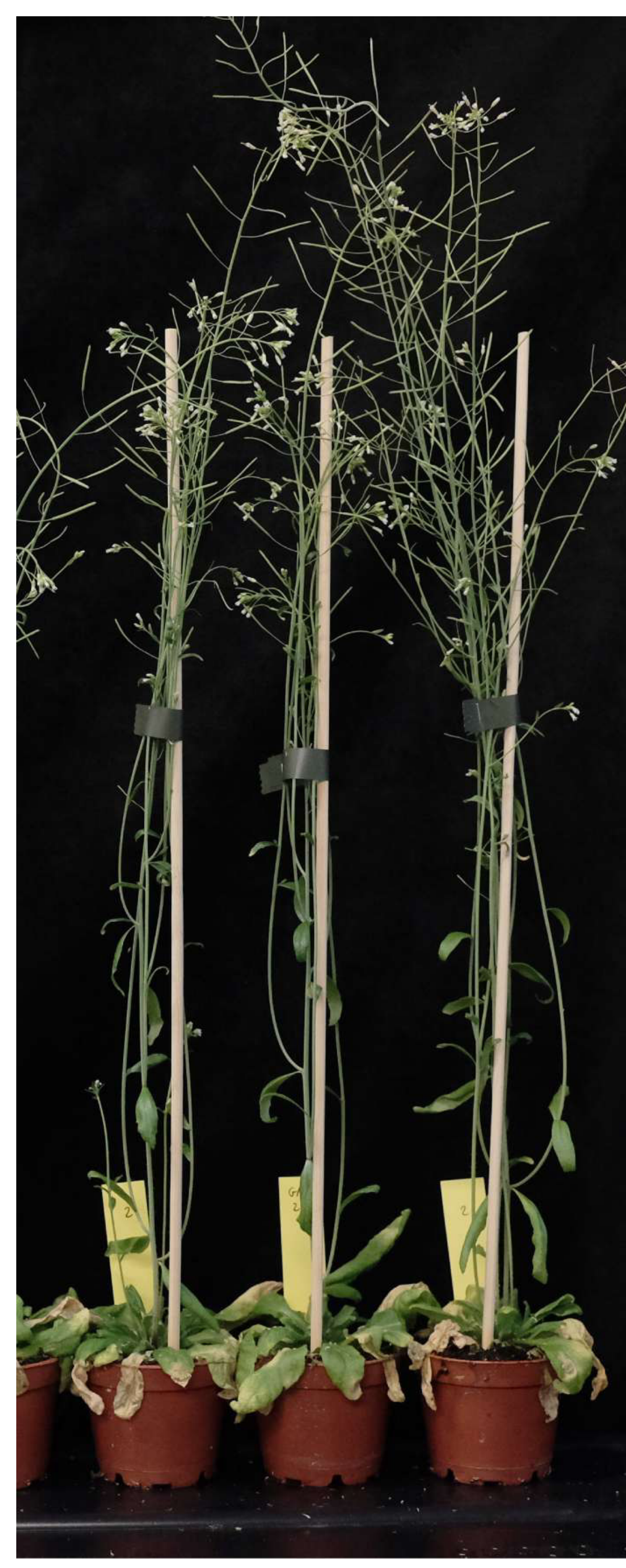

$g n^{s} G N_{\text {pro }}: G N-G F P$

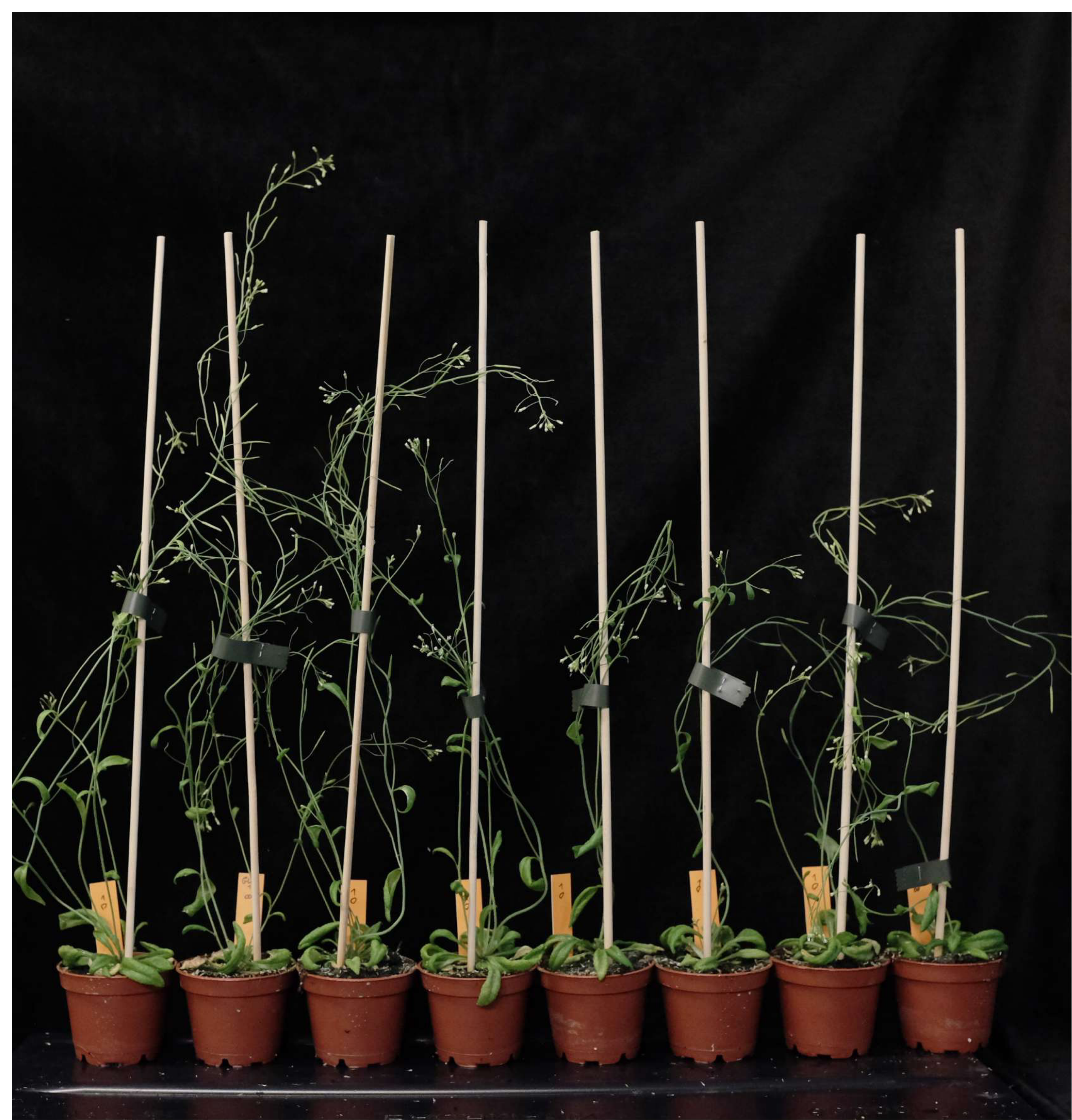

$g n^{s} G N_{p r o}: G L G-G F P$

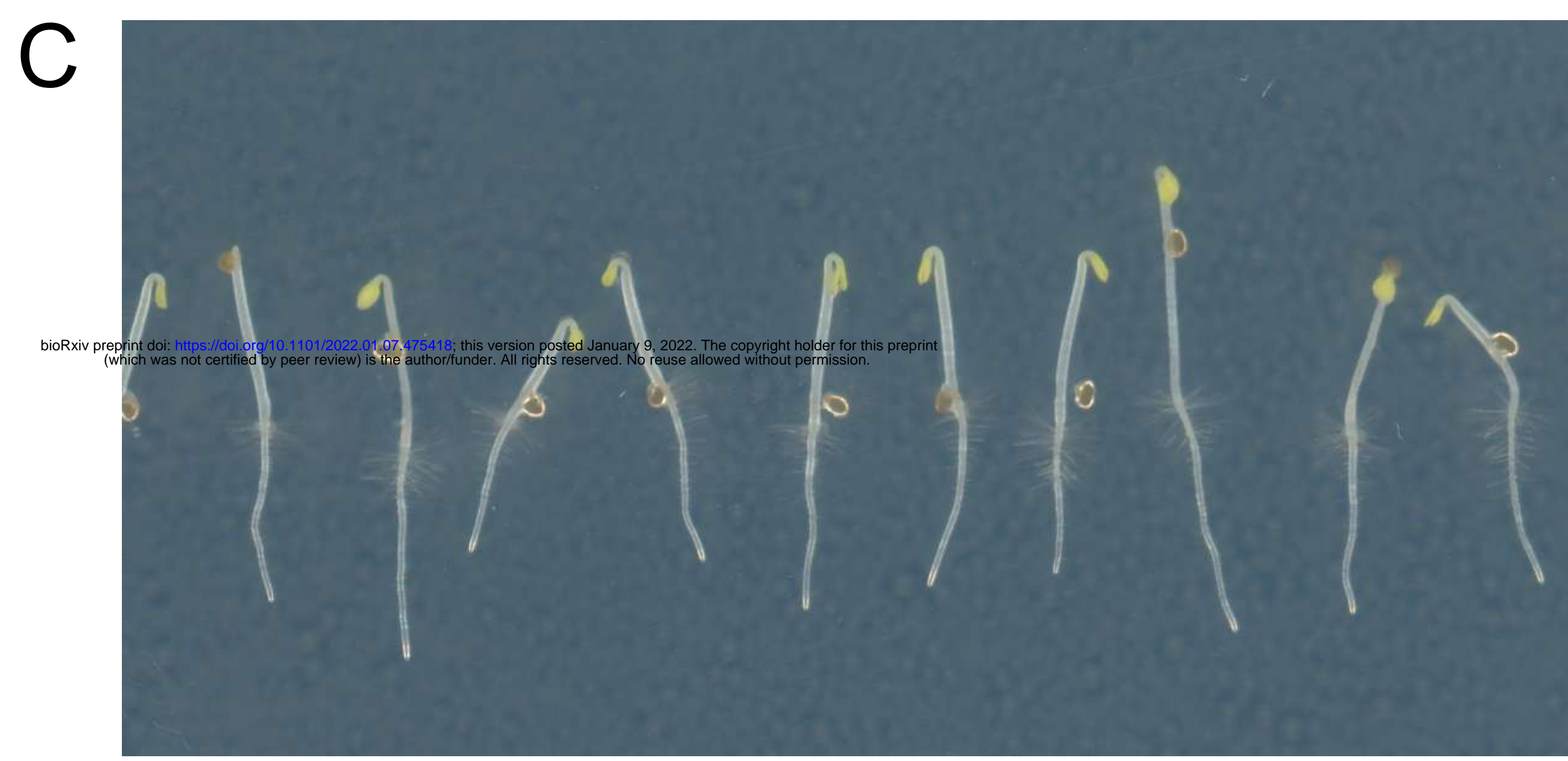

Col-0

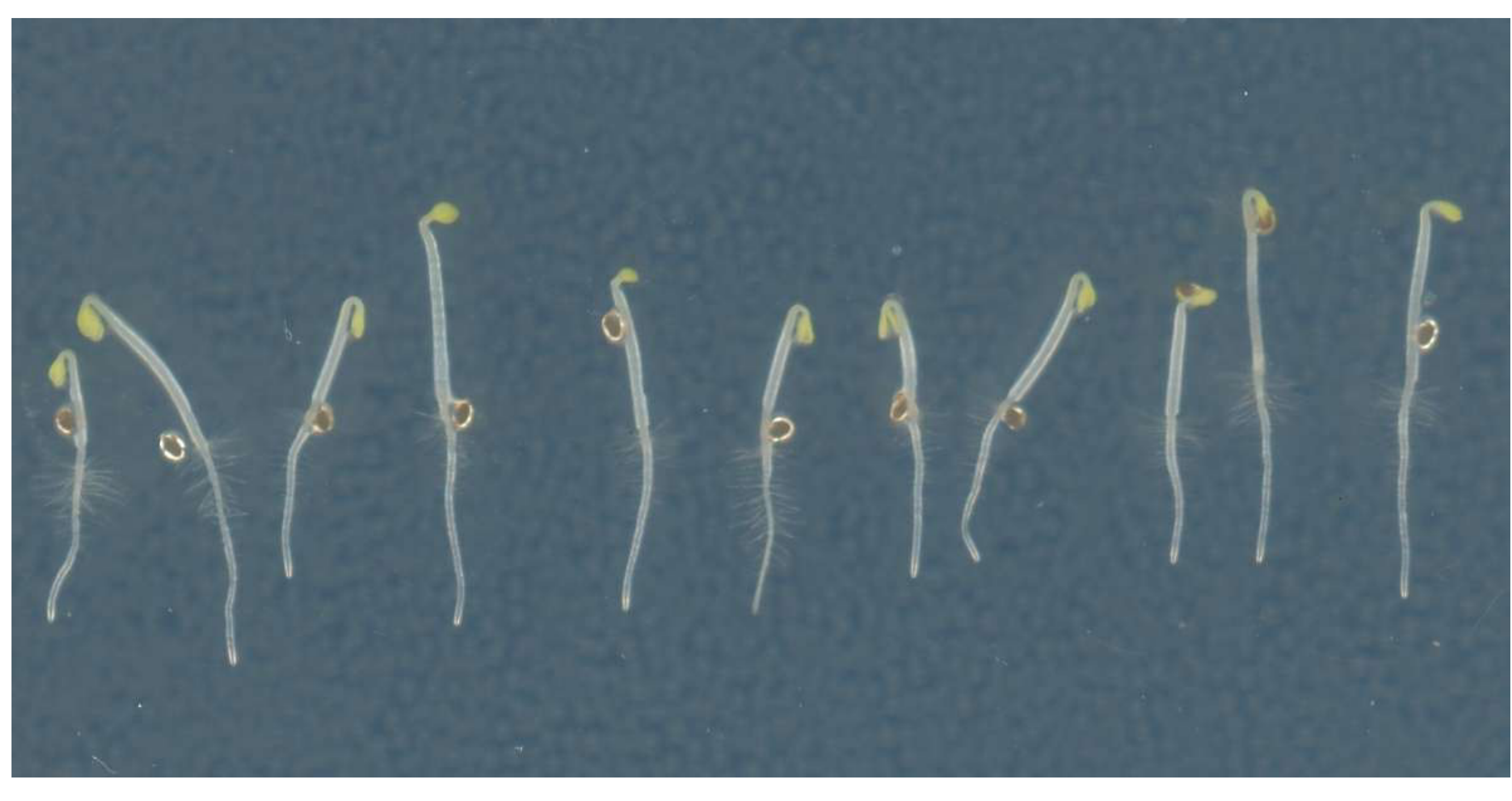

$g n^{s}$ GN $_{\text {pro }}: G N-G F P$ 


\section{Figure 7}
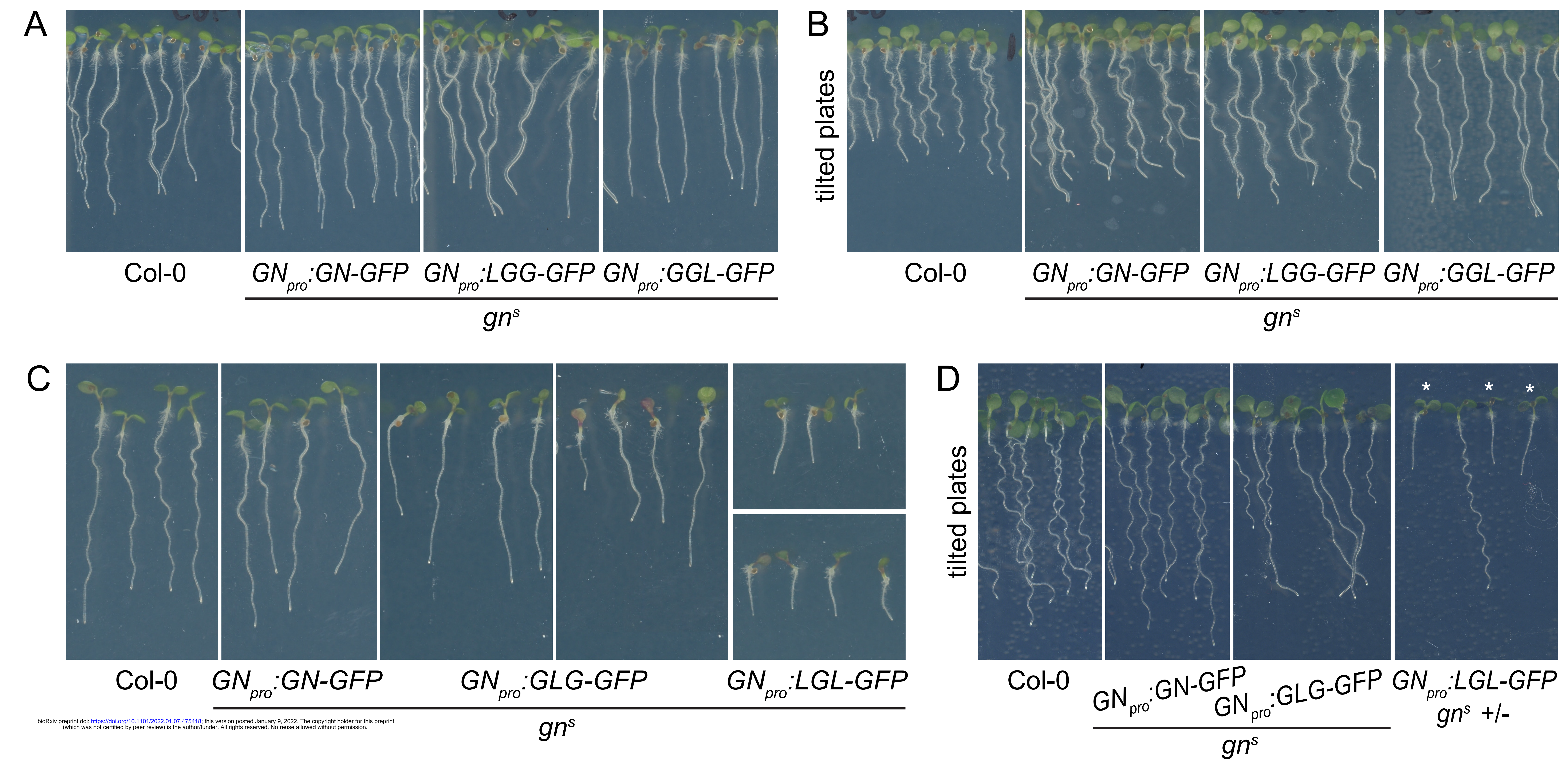

E

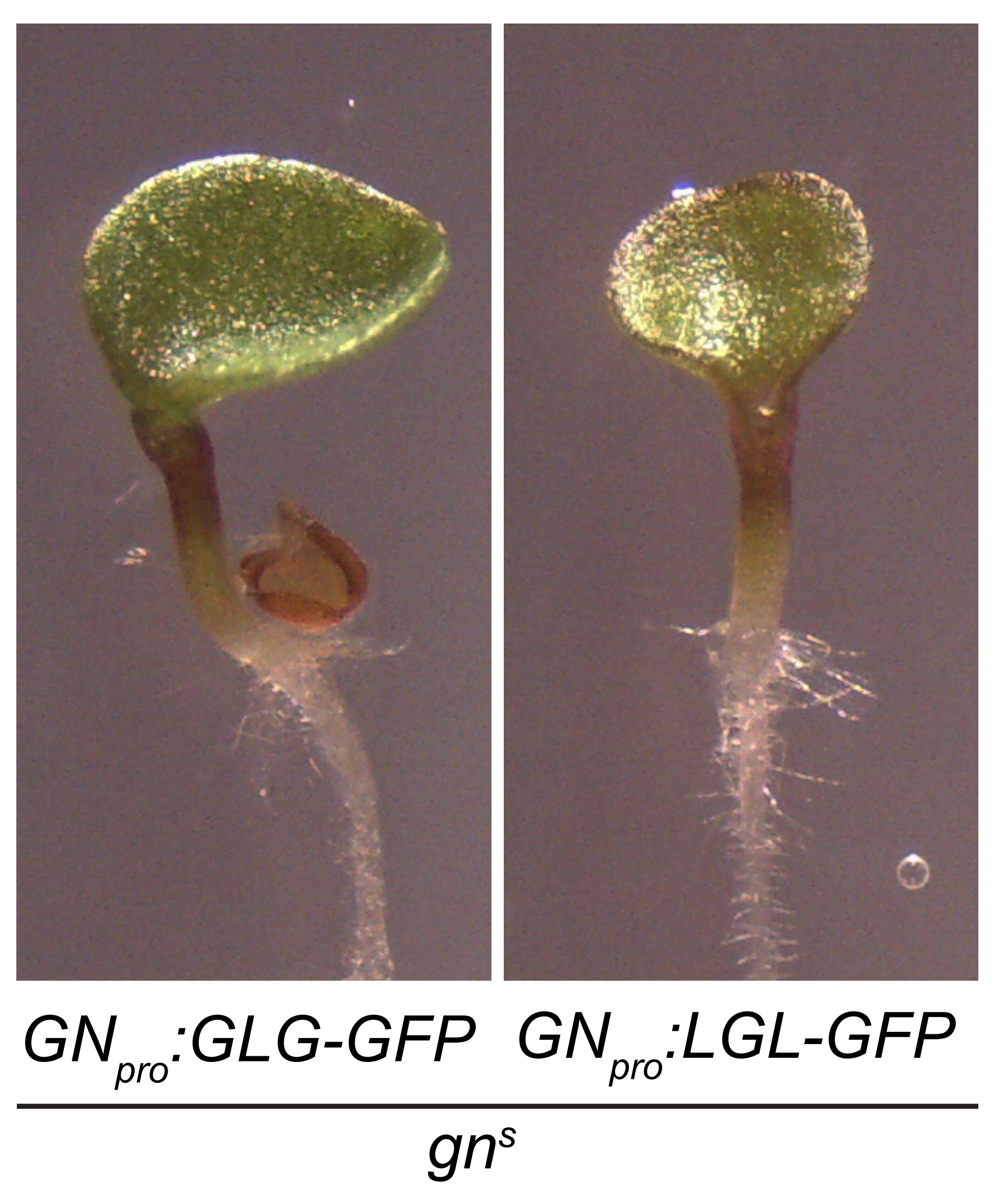

F

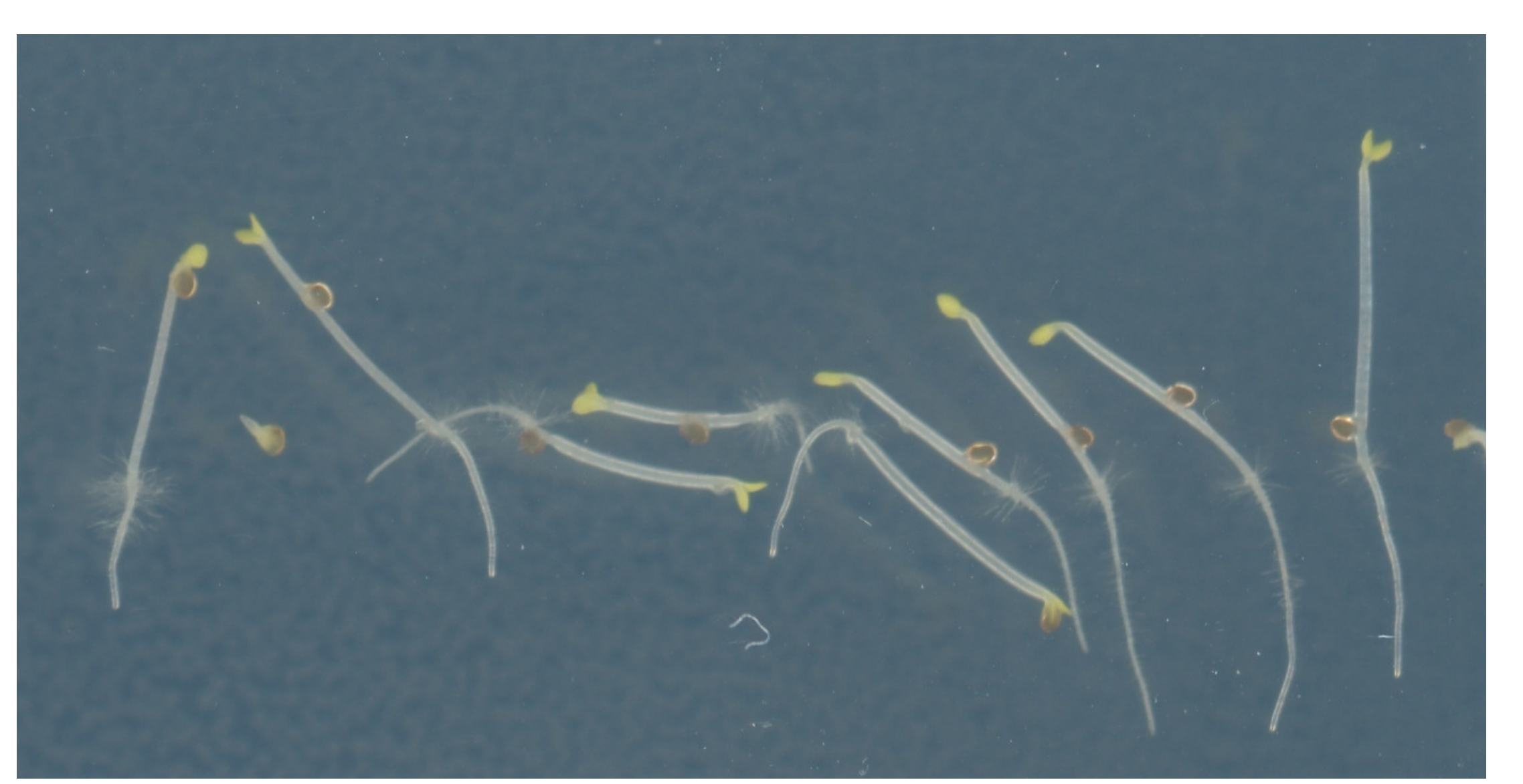

$g n^{s} G N_{\text {pro }}: G L G-G F P$

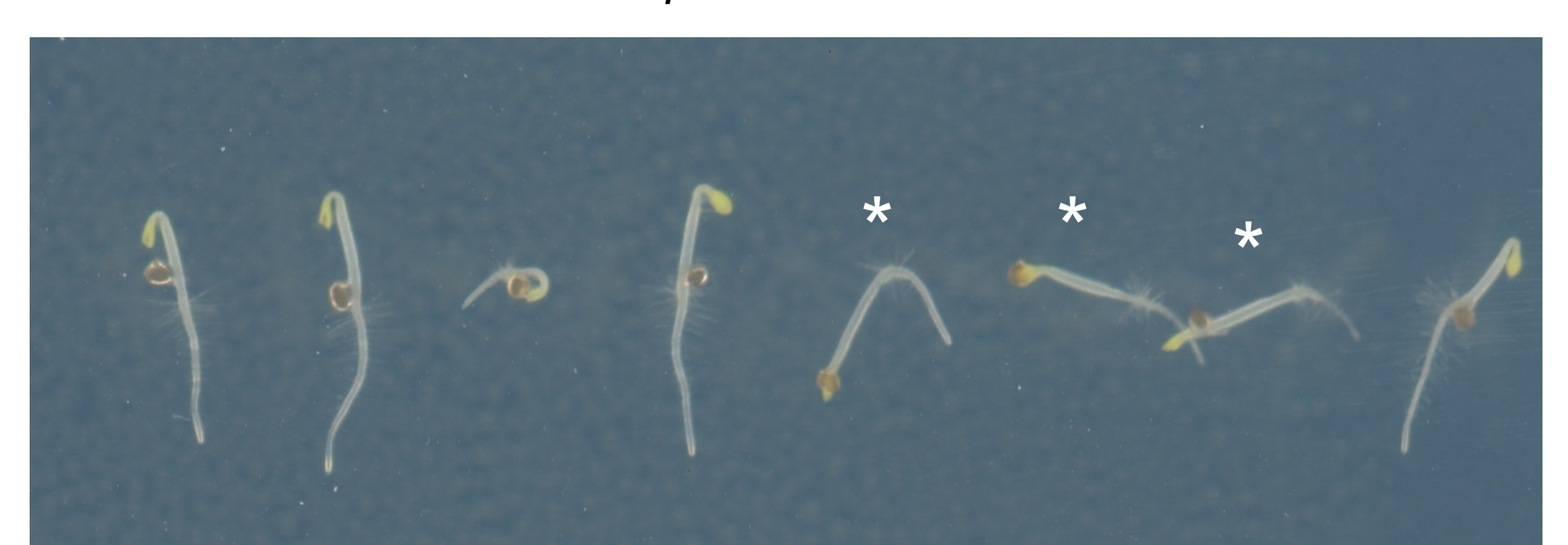

$g n^{s}+/-G N_{\text {pro }}: L G L-G F P$
G

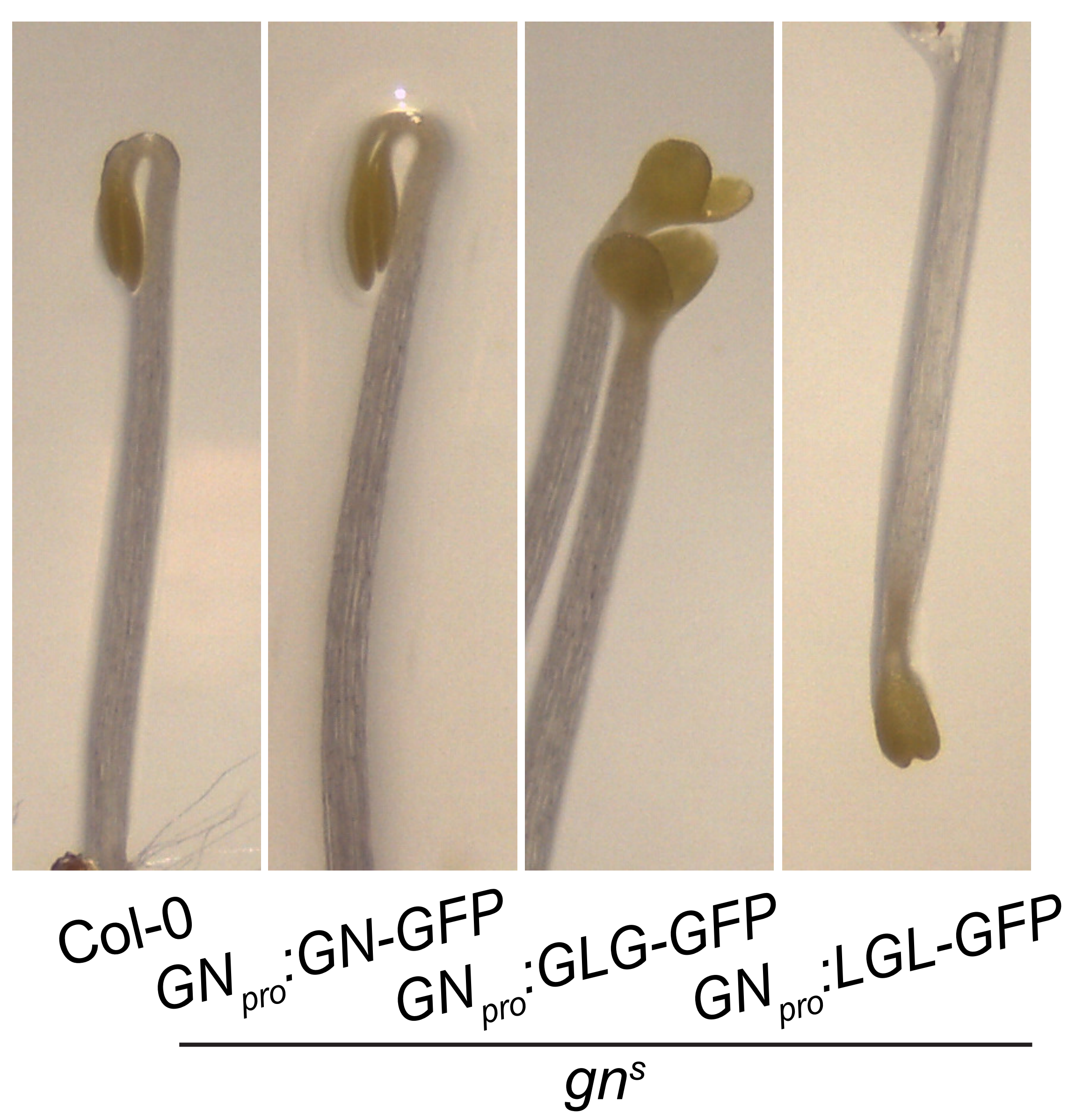


Figure 7-figure supplement 2
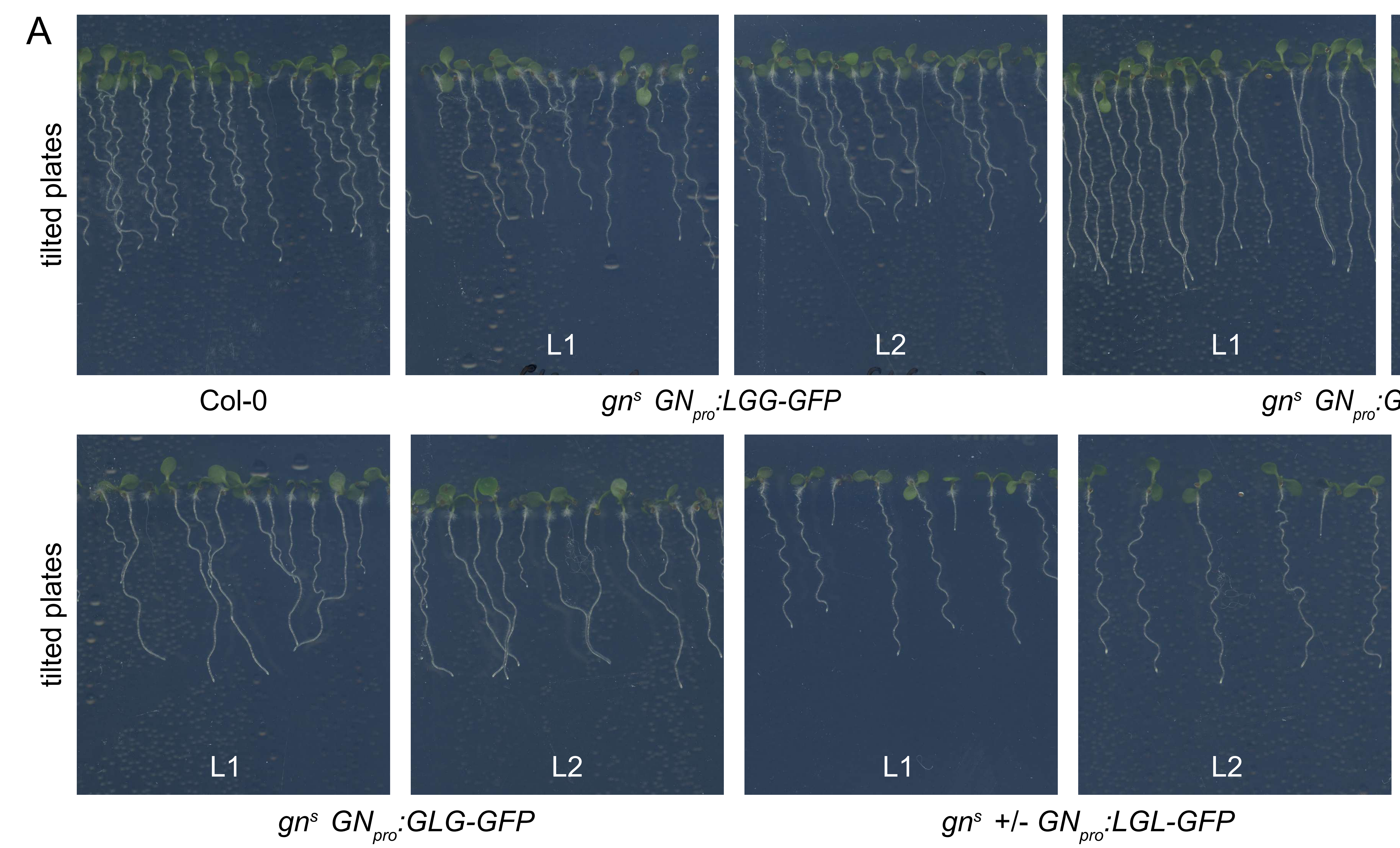

B

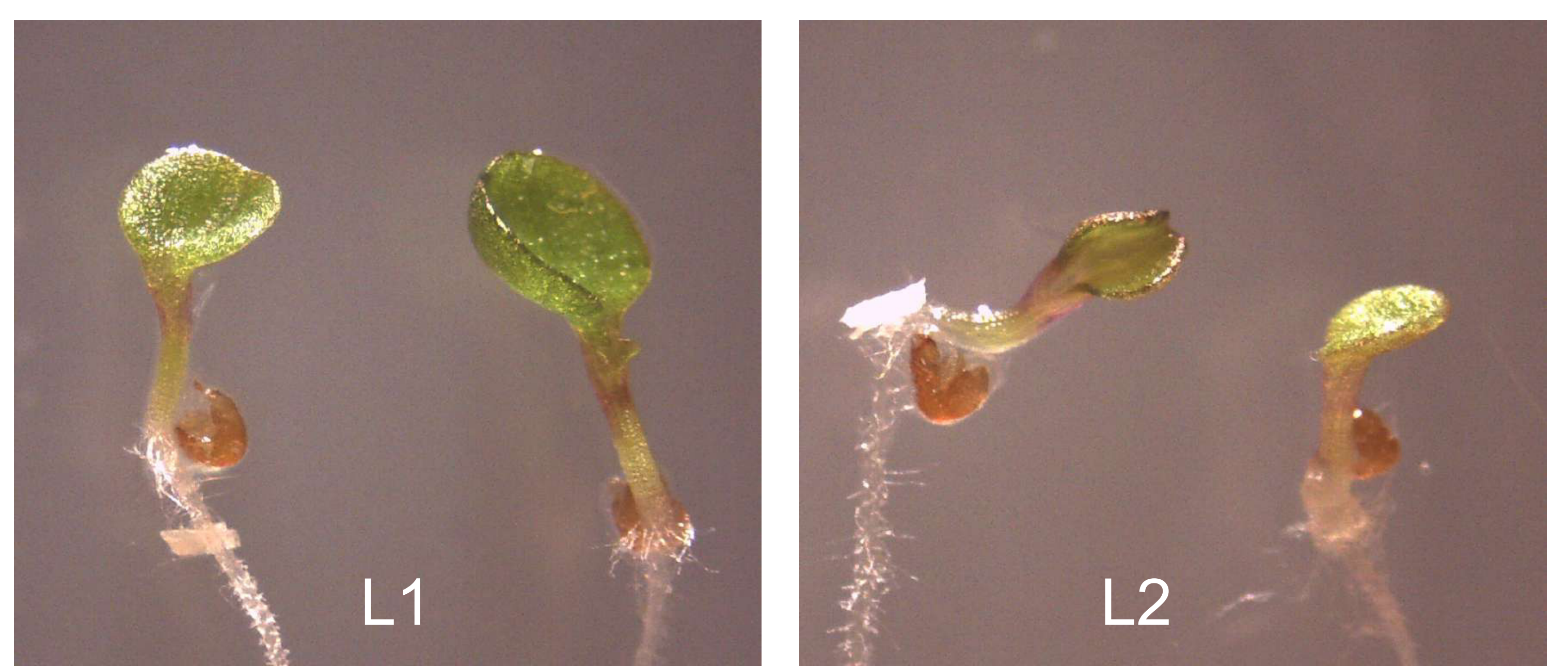

$g n^{s} G N_{p r o}: G L G-G F P$

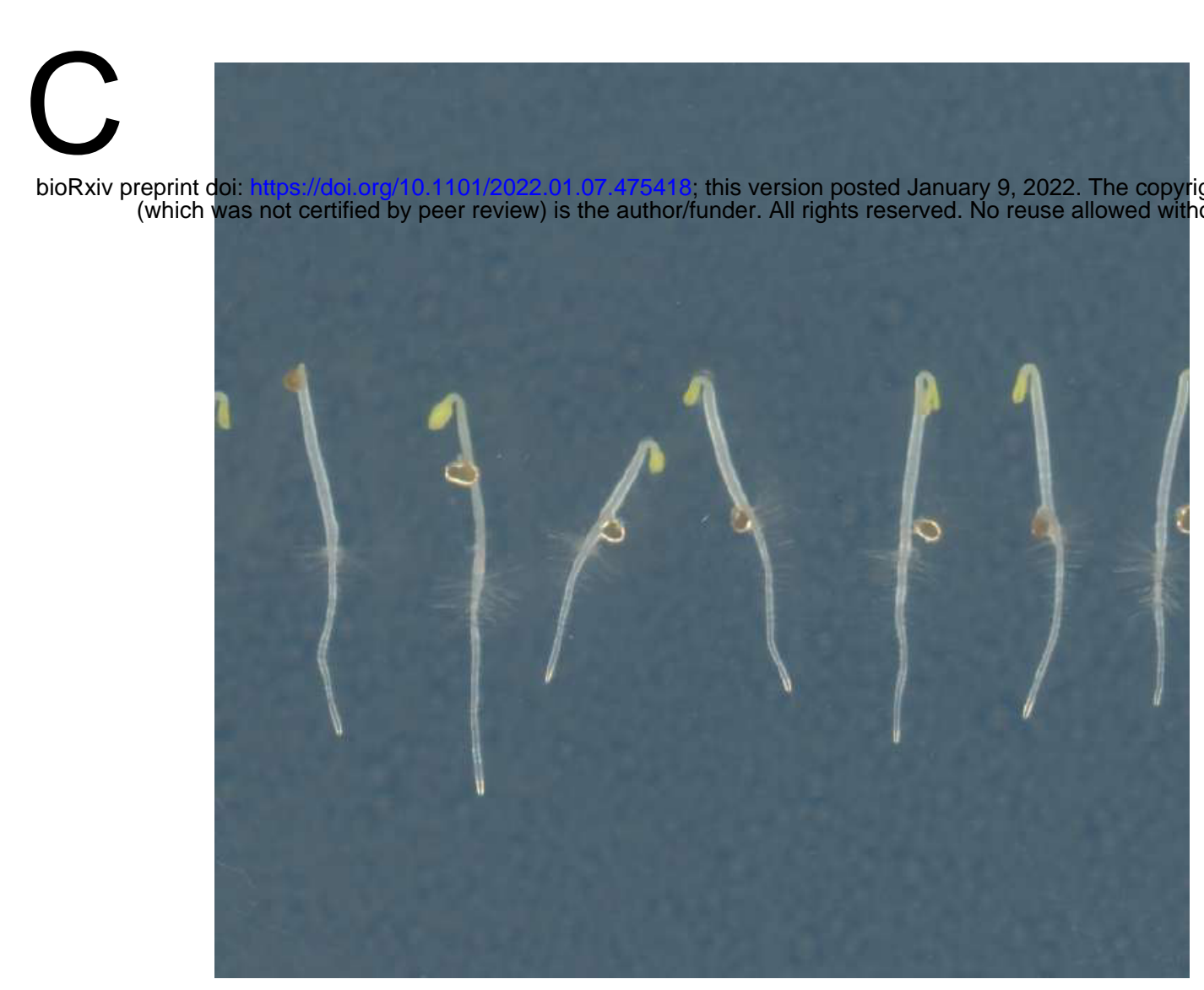

Col-0

D

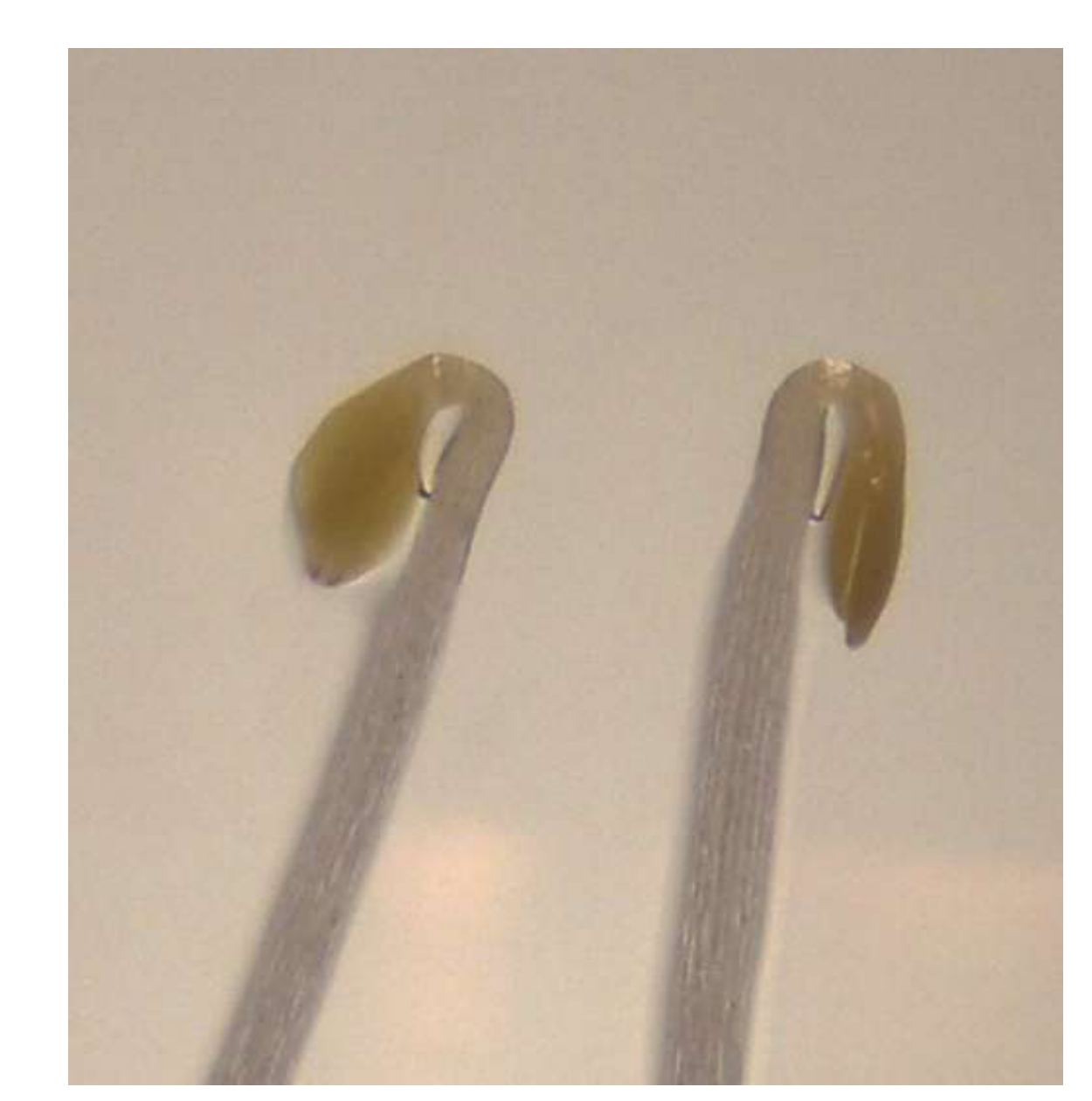

Col-0

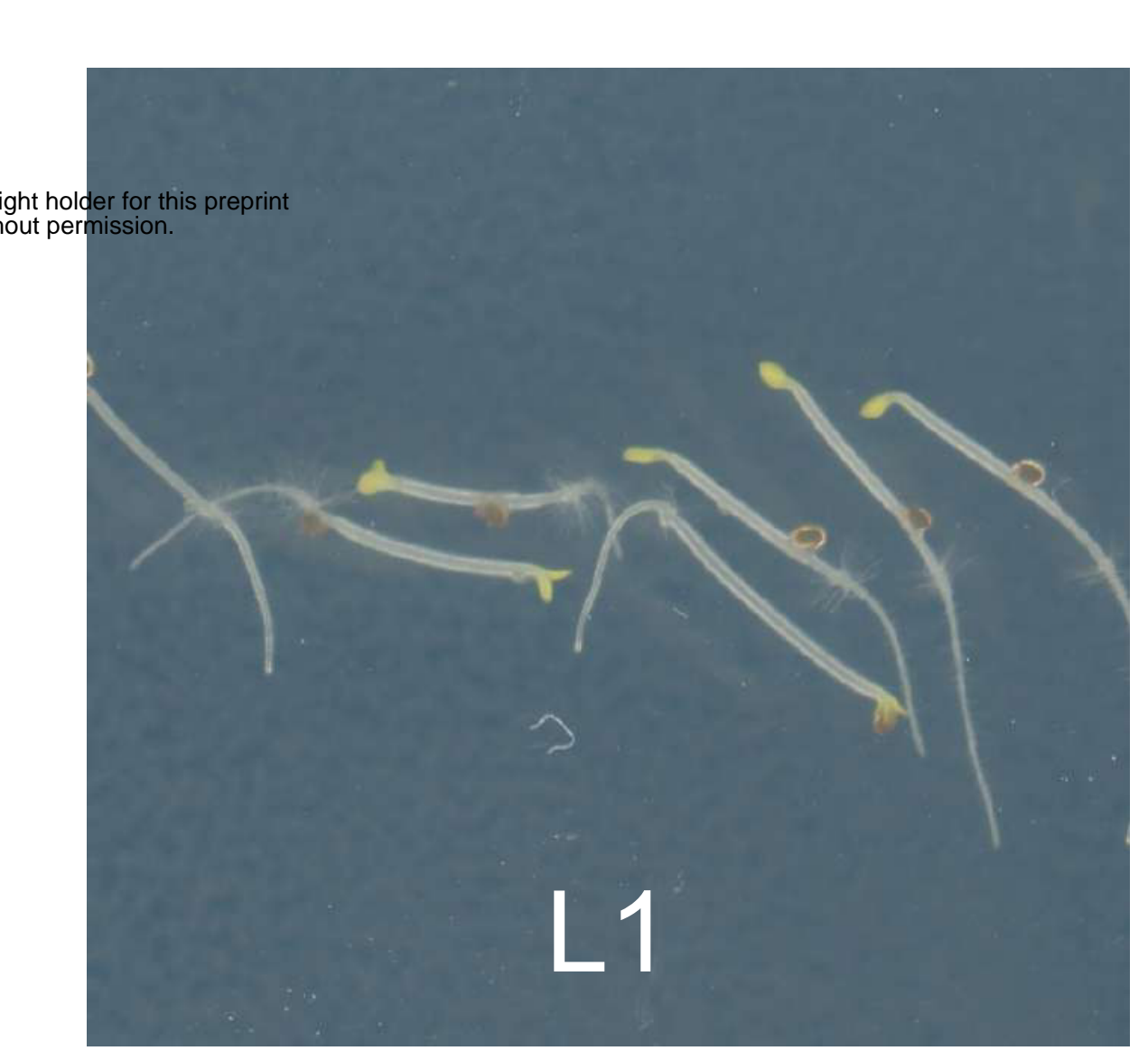

$g n^{s} \quad G N_{p r o}: G L G-G F P$

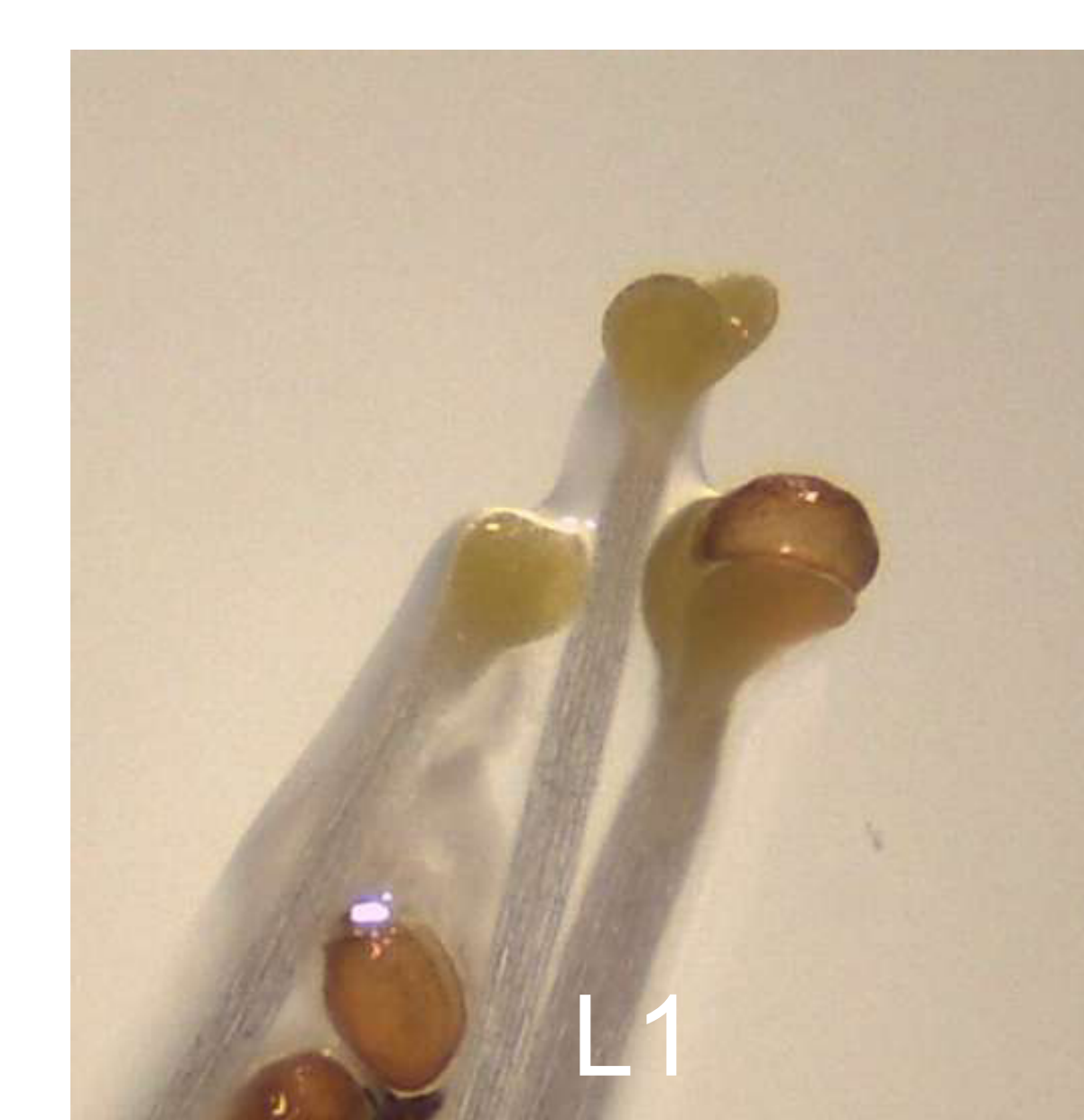

$g n^{s} G N_{p r o}: G L G-G F P$
L1

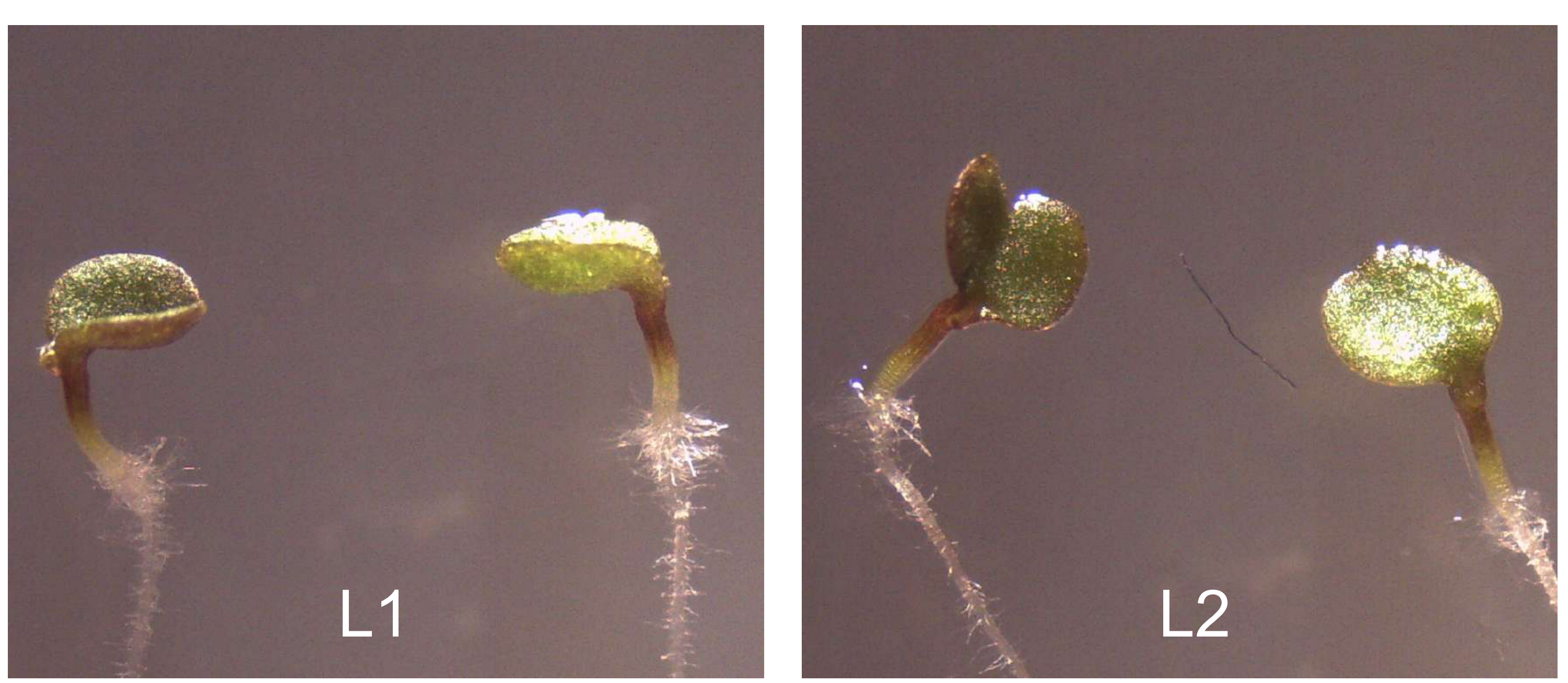

$g n^{s}$ GN $_{\text {pro }}: L G L-G F P$

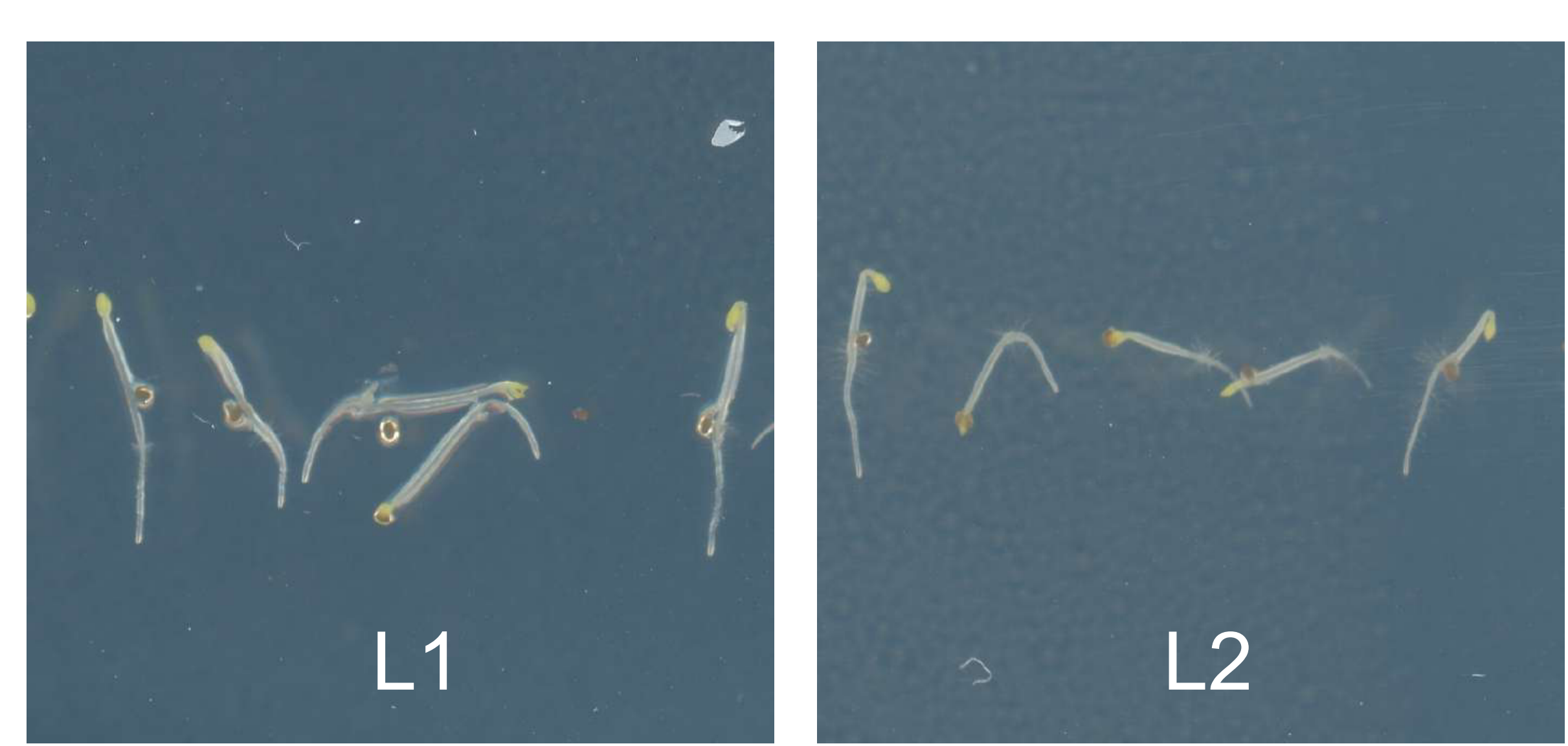

$g n^{s}+/-G N_{p r o}: L G L-G F P$

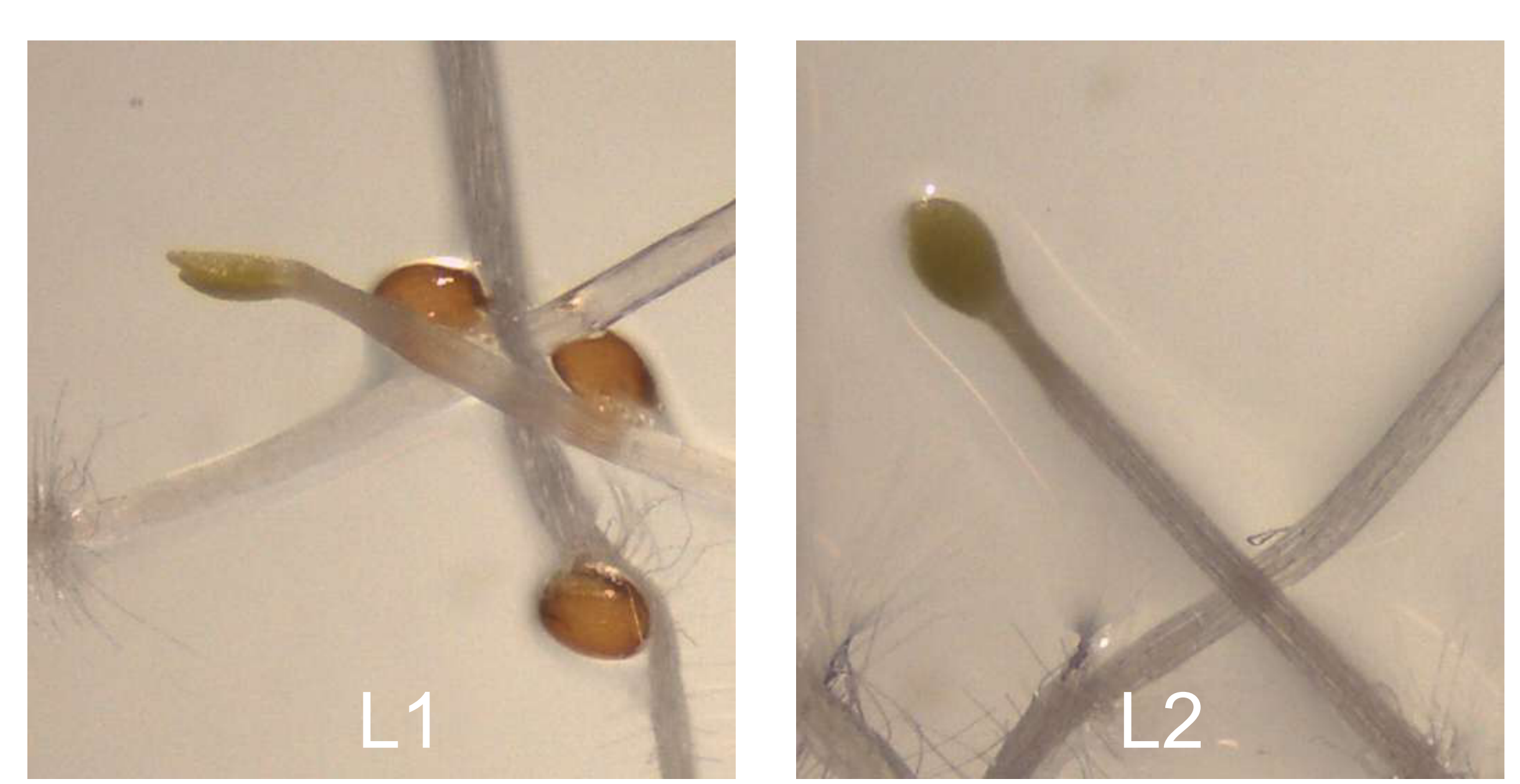

$g n^{s}+/-G N_{p r o}: L G L-G F P$ 
Figure 8-figure supplement 1
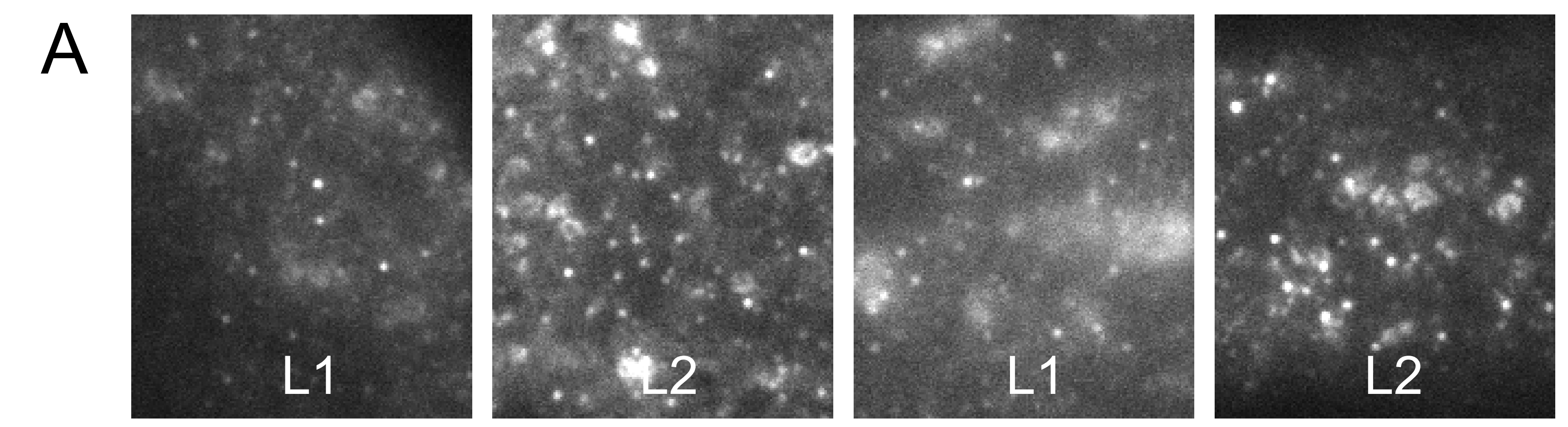
$G N_{p r o}: L G G-G F P$ $G N_{\text {pro }}: G G L-G F P$

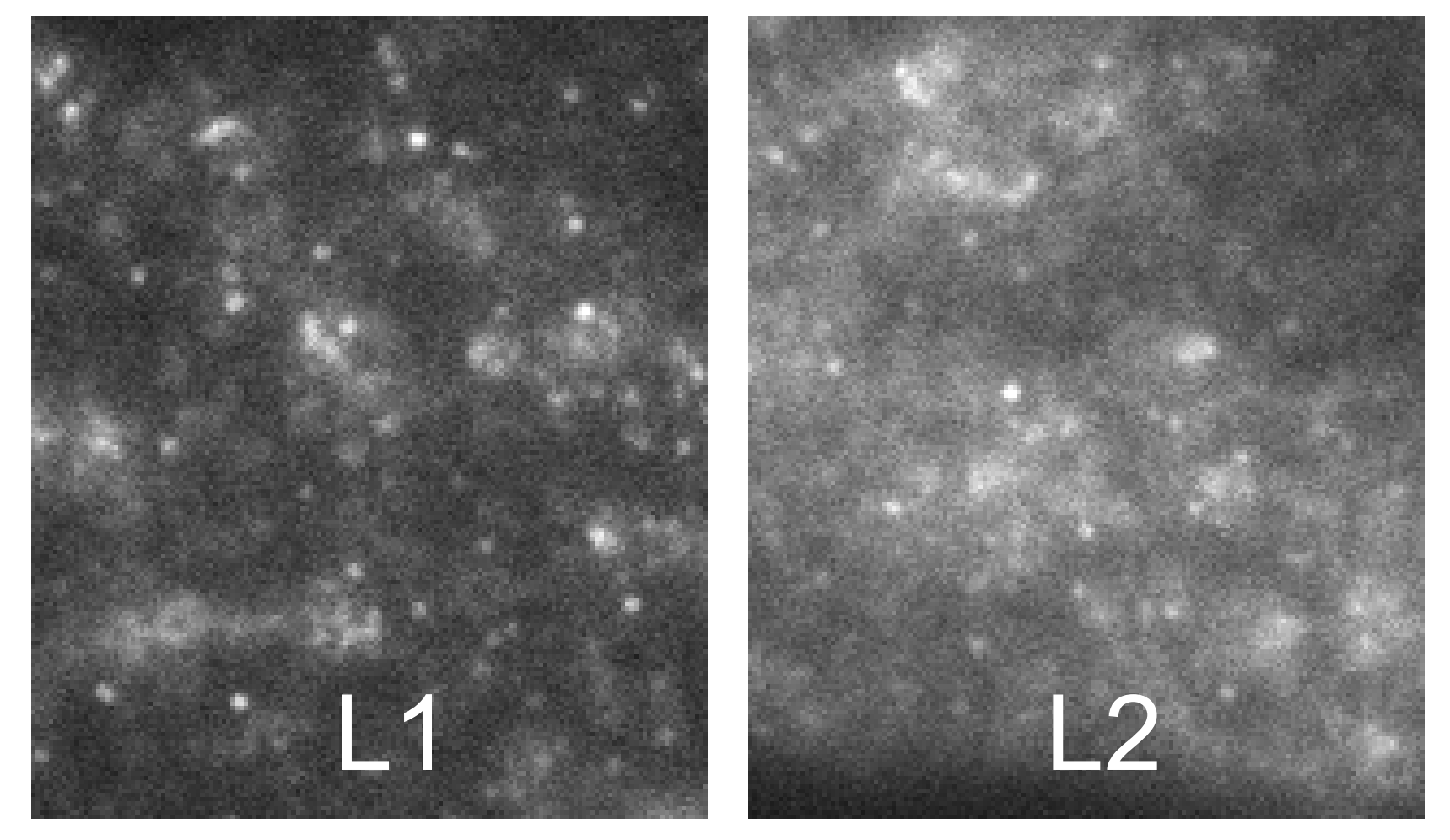

$G N_{\text {pro }}: G L G-G F P$

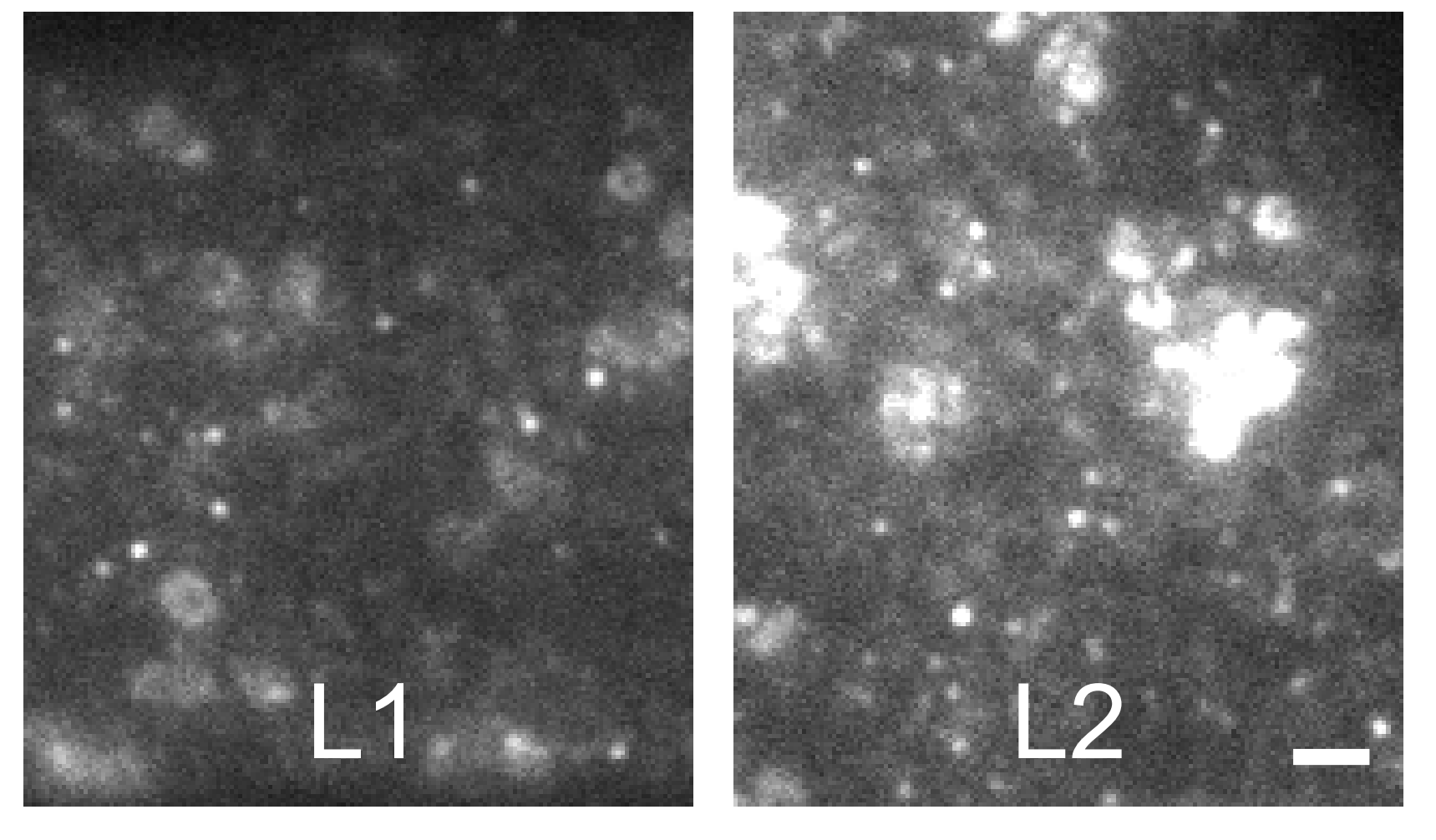

$G N_{p r o}: L G L-G F P$

$g n^{s}$

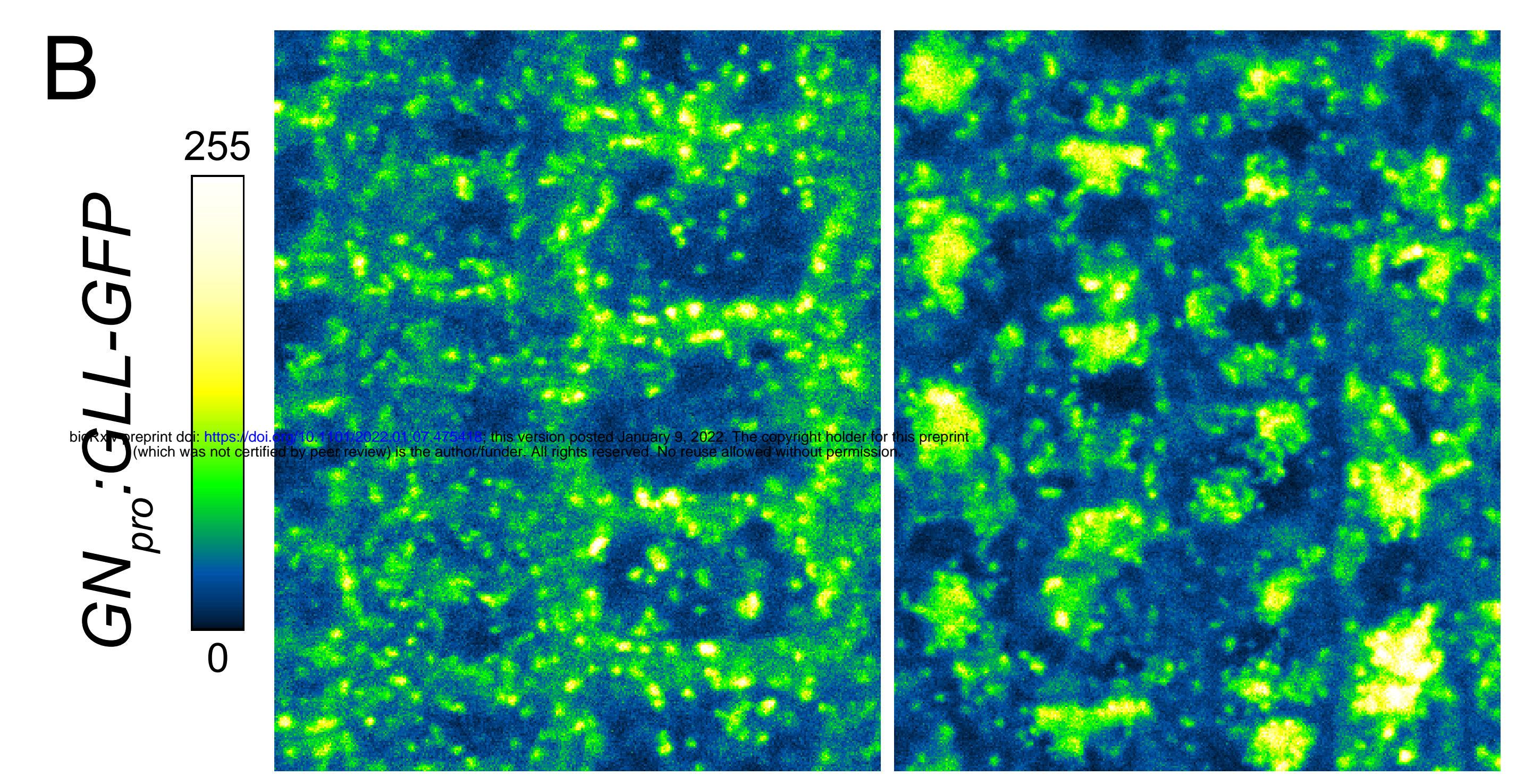

mock

BFA $50 \mu \mathrm{M} 1 \mathrm{~h}$

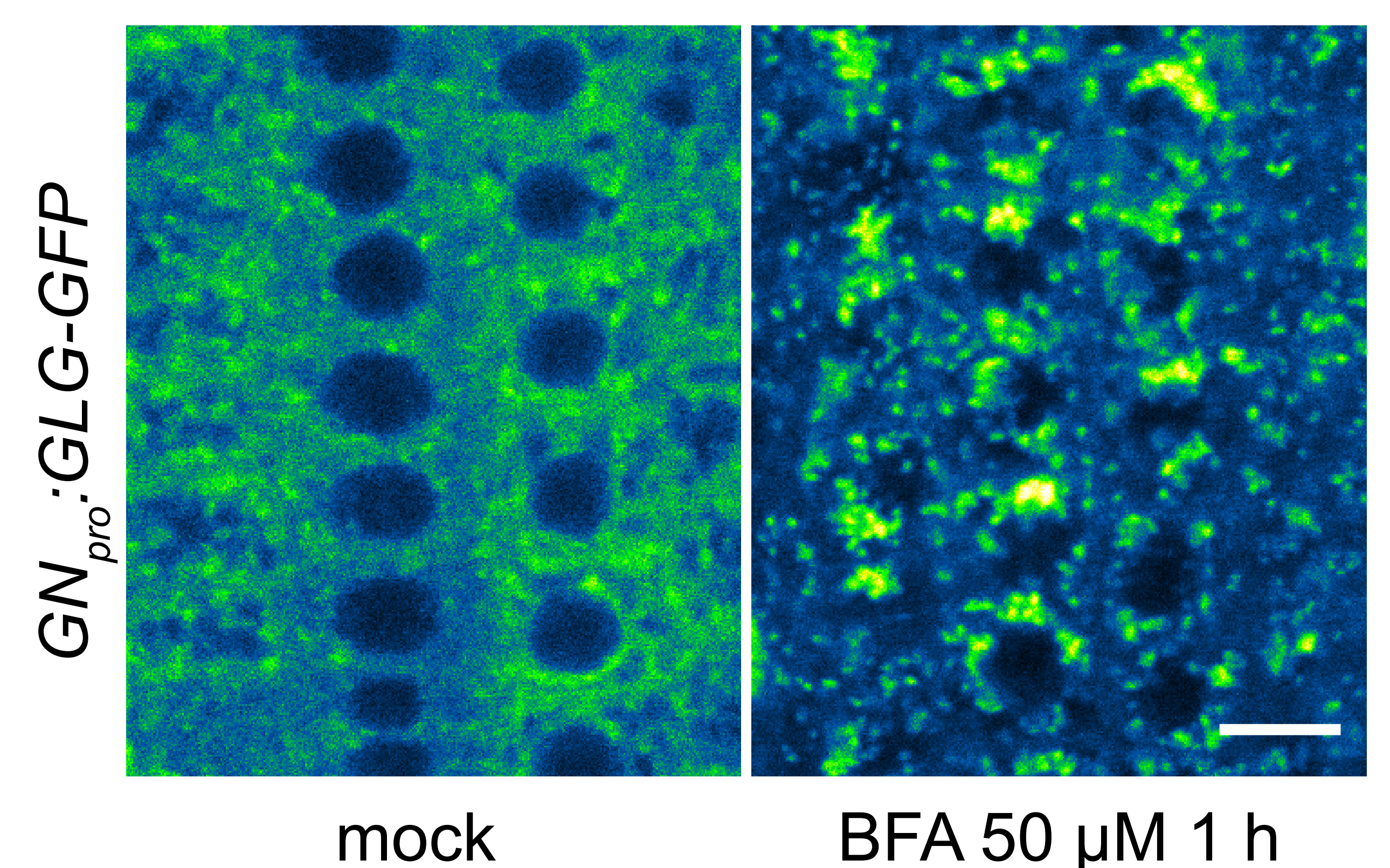

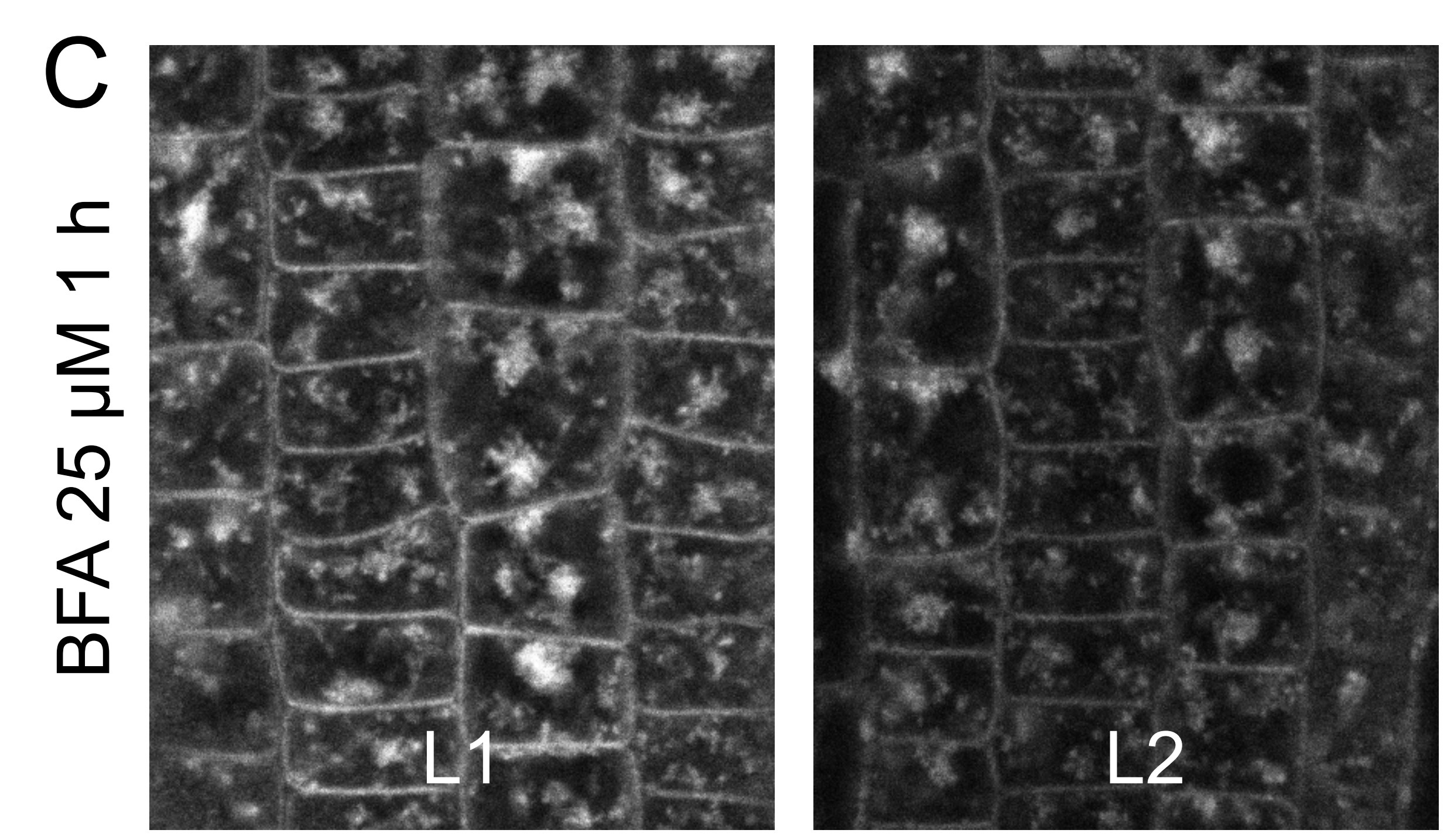

$G N_{p r o}: L G G-G F P$

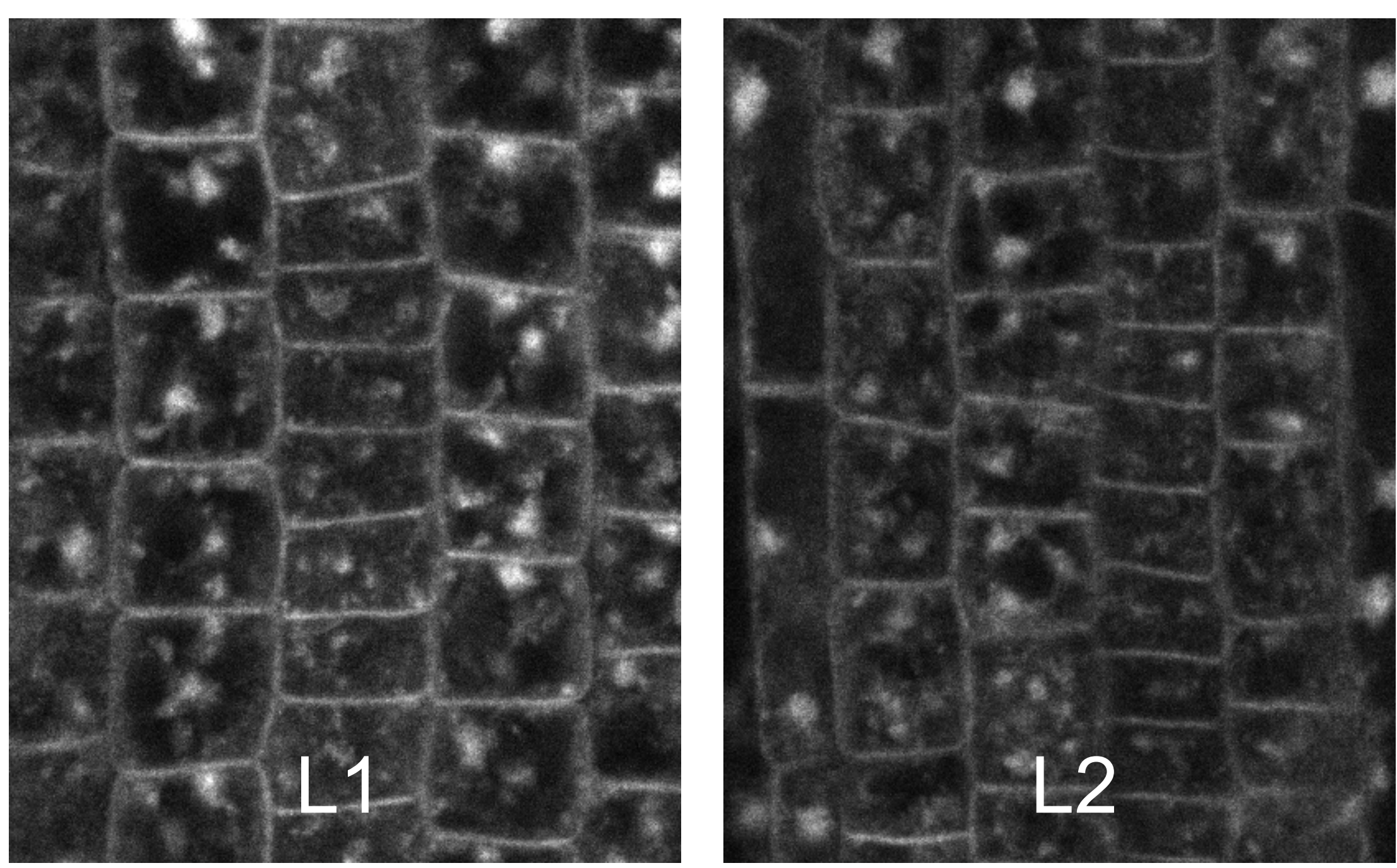

$G N_{\text {pro }}: G G L-G F P$
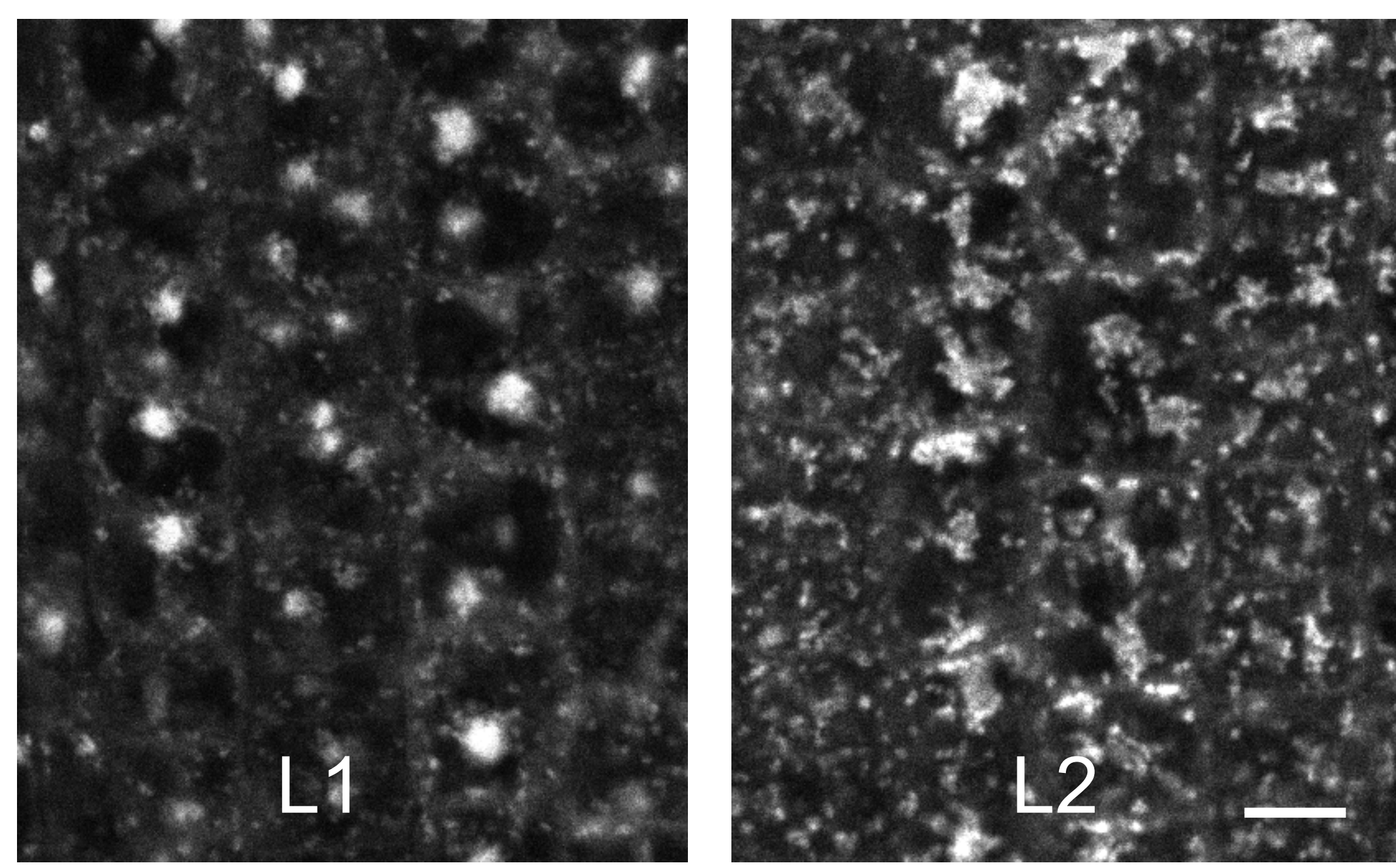

$G N_{\text {pro }}: L G L-G F P$ 